
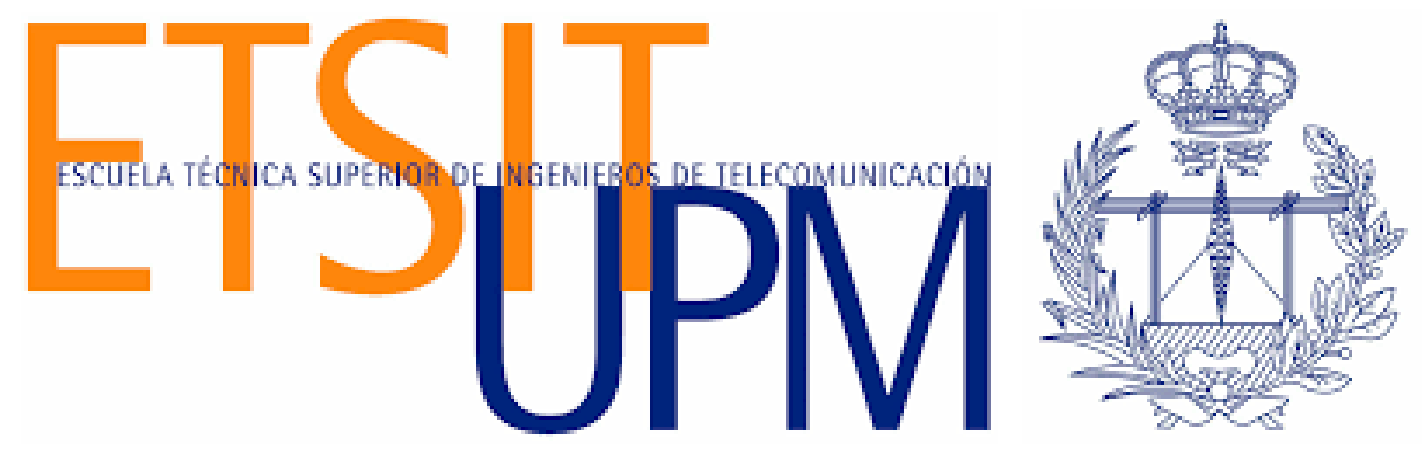

\title{
AUTOMATIC PSYCHOMOTOR FUNCTION QUANTIFICATION IN PARKINSON'S DISEASE VIA NATURAL INTERACTION WITH DIGITAL DEVICES
}

Author:

Teresa ARroyo-GALLEGO
Supervisor:

Dr. María Jesús LEDESMA-CARBAYO

Dr. Luca GIANCARDO

Mentor:

Dr. Martha GRAY

\section{Universidad POLITÉNICA DE MADRID PHD THESIS}

Departamento de Ingeniería Electrónica

Biomedical Image Technologies 



\title{
UNIVERSIDAD POLITÉNICA DE MADRID
}

\author{
Abstract \\ Departamento de Ingeniería Electrónica \\ Doctor of Philosophy

\begin{abstract}
AUTOMATIC PSYCHOMOTOR FUNCTION QUANTIFICATION IN PARKINSON'S DISEASE VIA NATURAL INTERACTION WITH DIGITAL DEVICES
\end{abstract}

by Teresa ARroyo-GALLEgo

This PhD thesis focuses on the study and development of new diagnostic techniques for evaluating psychomotor degradation in subjects affected by neurodegenerative diseases and movement disorders, specifically Parkinson's disease. We propose a novel method based on leveraging user-device interaction as a source of information to detect and monitor psychomotor impairment associated with this type of pathology. The objective is to collaborate in the development of a new tool for the quantification of psychomotor damage that could be used in conjunction of current clinical standards.

The proposed method for evaluating user-device interaction is based on capturing and analyzing the temporal information associated with each keystroke during a typing routine, whether on physical keyboards or touch screens. The main research tasks have focused on the implementation and evaluation of different algorithms for extracting biometric information related to the psychomotor function from typing activities that are part of users' daily routine.

The activities have been developed within the framework of a study on Parkinson's disease as part of a scientific project of the M+Vision consortium ("neuroQWERTY"). In this context, the goal is to develop a tool that provides an objective evaluation of the psychomotor function, while being accessible and unobtrusive to the user. Based on the analysis of the timing patterns built from fingers interactions with mechanical and touchscreen keyboards, we developed a series of algorithms that introduce a collection of digital markers to generate computational outcomes measurements for the management of PD. Specifically, we have validated the feasibility of this approach to detect early signs of PD via mechanical and touchscreen typing. Also, we have developed an algorithm to identify response to medication through the analysis of longitudinal patterns of mechanical keyboard typing.

These findings have a huge impact on the monitoring of the disease, since it brings us closer to an automatic quantification of the progress of the symptoms. Having detailed information on the evolution of the disease is crucial to progress towards increasingly personalized therapies, as well as improving our knowledge about the effect of these therapies and, consequently, promoting more efficient drug development. 



\section{Acknowledgements}

I would like to start by acknowledging all the individuals that have contributed and made this work possible.

First and foremost, I would like to thank my Thesis directors Luca and Chus for their guidance and support during this journey. I have learnt a lot from you during these years.

A big thank you to the rest of the neuroQWERTY team; Álvaro, Ian, Carlos, Michele, Verónica and Paloma, for their incredible work during these years. It has been a pleasure to be part of this team.

I would also like to thank the M+Vision faculty for their feedback and lessons during my time at MIT. Especially I would like to thank Martha for her invaluable support and continuous willingness to help since the very beginning of this project an even after leaving the lab.

Thank you also to the team at nQ Medical, especially to Rich and Mark. It is also thanks to them that this project is now a reality.

To all study participants, this research would not exist without their bravery and dedication to clinical research.

To all my friends, and a special mention to those that have been pushing me to keep going when things got a little harder. In particular, to my Boston roommates, Peter and Carlos, for keeping me from procrastinating when I had to write.

Lastly and most importantly, thank you to my family, specially my parents and sisters (also Mandi). You make life so much easier and beautiful with your unconditional love and support.

THANK YOU! 



\section{Contents}

$\begin{array}{ll}\text { Abstract } & \text { iii }\end{array}$

$\begin{array}{lll}\text { Acknowledgements } & \text { v }\end{array}$

1 Introduction $\quad \mathbf{1}$

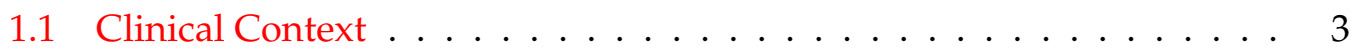

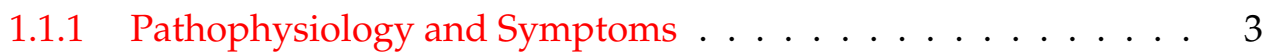

1.1.2 Current Clinical Standard . . . . . . . . . . . . . . 6

1.2 Statement of Need . . . . . . . . . . . . . . . . . 9

1.2.1 The Impact in Parkinson's Disease . . . . . . . . . . . . . . 9

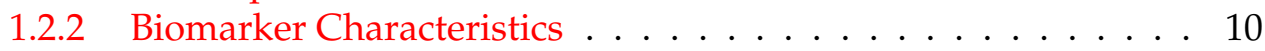

1.3 Opportunity and Impact . . . . . . . . . . . . . . . . . . . . . . . . . . . 11

1.3.1 The Opportunity of Early Detection . . . . . . . . . . . . 12

1.3.2 The Opportunity of Precise Monitoring . . . . . . . . . . 13

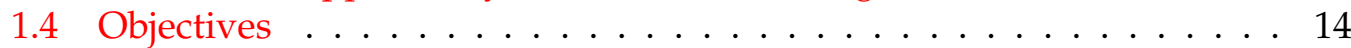

1.5 Document Organization .................... 15

2 State of the Art $\quad 17$

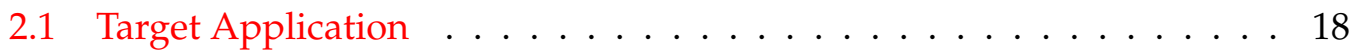

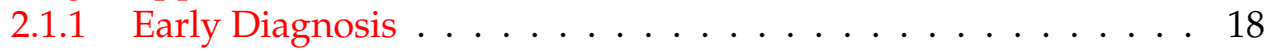

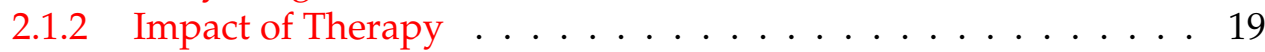

2.1.3 Remote Monitoring ................ 20

2.2 Measured Clinical Feature . . . . . . . . . . . . . . 20

2.2.1 Motor Domain . . . . . . . . . . . . . . 21

Gait and Posture . . . . . . . . . . . . . . . . . . . . . 21

Bradykinesia and Rigidity . . . . . . . . . . . . . 22

Tremor ................... 23

Levodopa-Induced Dyskinesia . . . . . . . . . . 23

2.2.2 Non-Motor Domain . . . . . . . . . . . . . . . 24

2.2.3 Multi Domain . . . . . . . . . . . . . . . . 25

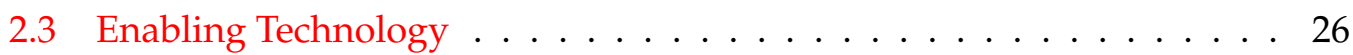

2.4 Patient Involvement . . . . . . . . . . . . . . . 28

3 Scientific Rationale $\quad 29$

3.1 Solution Context and Advantages . . . . . . . . . . . . . . . . 29

3.2 The Kinematics of Typing . . . . . . . . . . . . . . . . . 33

3.3 The Connection with Parkinson's Disease . . . . . . . . . . . . . 37

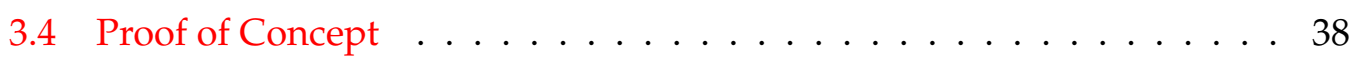

4 Keyboard Typing as an Indicator of Early Parkinson's Disease 41

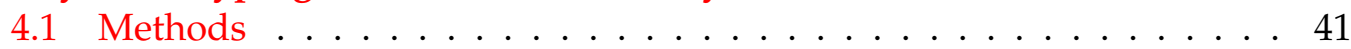

4.1.1 The neuroQWERTY index (nQi) . . . . . . . . . . . . 43

Signal Representation ............... 43 
Ensemble regression . . . . . . . . . . . . . . . . 45

4.1.2 Real-world data collection . . . . . . . . . . . . . . . . 45

4.1 .3 Analysis . . . . . . . . . . . . . . . . . . . . . 49

4.2 Results . . . . . . . . . . . . . . . . . 51

4.3 Discussion . . . . . . . . . . . . . . . . . 54

5 Touchscreen Typing as an Indicator of Early Parkinson's Disease 57

5.1 Methods ....................... 58

5.1 .1 Data Acquisition . . . . . . . . . . . . . . 58

5.1 .2 Data Analysis . . . . . . . . . . . . . . . . . . 58



Feature Extraction . . . . . . . . . . . . . . 61

Evaluation Methodology . . . . . . . . . . . 66

5.2 Results . . . . . . . . . . . . . . . . . 66

5.3 Discussion . . . . . . . . . . . . . . . . 71

$\begin{array}{lll}6 & \text { Response to medication } & \mathbf{7 3}\end{array}$

6.1 Methods . . . . . . . . . . . . . . . . . . . 74

6.1 .1 Study design . . . . . . . . . . . . . . 74

6.1 .2 Motor change definition . . . . . . . . . . . . 76

6.1.3 Data availability and group distribution . . . . . . . . . 77

6.1 .4 Analysis of in-clinic data . . . . . . . . . . . . . . . . 78

Signal representation . . . . . . . . . . . . . 78

Univariate Analysis . . . . . . . . . . . . . . 78

6.1 .5 Analysis of At-Home Data . . . . . . . . . . . . . 80

Signal representation . . . . . . . . . . . . . . . . . . 80

Multivariate Analysis . . . . . . . . . . . . . . 81

Analysis methods . . . . . . . . . . . . . . . . . 83

6.2 Results . . . . . . . . . . . . . . . . . . . 85

6.3 Discussion . . . . . . . . . . . . . . . . . 85

7 Summary and Conclusions $\quad 91$

7.1 Hypothesis and Objectives Revisited . . . . . . . . . . . . . . . . . 91

7.2 Conclusions . . . . . . . . . . . . . . . . 93

7.3 Impact and Future Work . . . . . . . . . . . . . . . 93

$\begin{array}{lr}\text { Bibliography } & 95\end{array}$ 


\section{List of Figures}

1.1 Braak Stages for Parkinson's pathology. Braak et al. developed a staging system that characterizes disease progression. This system is divided into six different stages, with each stage being attributed to abnormal pathology in particular neurological structures. In terms of symptomatology, the type and severity of symptoms is correlated to progression through the Braak stages. Early stages are characterized by non-motor symptoms. Motor symptoms are often displayed around the mid-stage state, and cognitive symptoms arise as later Braak stages are reached. Modified from (Braak et al., 2003). . . . . . . . .

1.2 Parkinson's disease progression and signs. Parkinson's manifests in a variety of symptoms. Among them, four those affecting the motor function are considered the cardinal signs of PD. Specifically, bradykinesia, tremor, rigidity, and postural instability. Even though PD is generally referred as a movement disorder, non-motor symptoms are also common and contribute to disability as the disease progresses. These can include mood disorders and depression, cognitive dysfunction, hyposmia and dysautonomia. Modified from (Poewe et al., 2017). .

1.3 MDS-UPDRS Score Sheet The Unified Parkinson's Disease rating scale (UPDRS) is the most commonly used scale in the assessment of PD. The MDS-UPDRS was published by the Movement Disorder Society (MDS) in 2007 as a revision of the original scale. It evaluates various aspects of Parkinson's disease including non-motor and motor experiences of daily living and motor complications. The MDS-UPDRS includes four subscales: Part I (non-motor experiences of daily living), Part II (motor experiences of daily living), Part III (motor examination) and Part IV (motor complications). (Goetz et al., 2008) . . . . .

1.4 Clinical representation of Parkinson's Disease. As a chronic progressive disease, PD has a pre-symptomatic interval; that is, a period during which the pathological process has begun, but motor signs required for the clinical diagnosis are absent or not detectable. By the time PD is diagnosed, neurodegeneration is very advanced, which limits the impact of existing drugs to relief the disease symptoms. The ability to identify PD in its preclinical stage may be critical in the development and eventual use of neuroprotective therapies. (Heinzel et al., 2016) . . . . . . . . . . . . . . . . . . 
2.1 Digital technologies in Parkinson's disease. In the absence of specific in-vivo biomarkers for diagnosis and disease progression monitoring, PD remains a clinically defined disease. In the last decade, a variety of tecnology-based objective measures (TOMs) have been explored to address this unmet medical need. Given the complexity of the disease, these technologies cover a diverse range of approaches, with different use case focus, symptom targets, enabling technology and level of patient involvement. . . . . . . . . . . . . . .

3.1 Cortical sensory homunculus. A pictorial representation of the anatomical divisions of the primary motor cortex and the primary somatosensory cortex. It represents the portion of the human brain directly responsible for the movement and exchange of sensory and motor information of the body. Areas of the body with more complex and numerous sensory or motor connections are represented as larger in the homunculus. This is the case for hands and fingers, as they control most tasks that require fine motor skills. (Anatomy Eamp; Physiology)

3.2 A graphical representation of keystroke dynamics variables. Different combinations of timing events of pressing and releasing keys in mechanical or touchscreen keyboards have been traditionally used as a biometric signature to identify an individual based on the manner and the rhythm of their typing. Some of the most common metrics used in this field are the hold time (time between pressing and releasing a key) and the flight time (time between pressing to consecutive keys.) . . . . . . . . . . . . . . . . . . . 35

3.3 Effect of sleep inertia in typing dynamics. Examples showing the hold time distribution matrices in rested subjects compared with a state of sleep inertia. (Giancardo et al., 2015) . . . . . . . . . . . . . 38

4.1 Pipeline of the algorithm to generate the neuroQWERTY score (nQi) from the hold time (HT) series. (1) The HT time events are split by non overlapping 90.seconds windows to create the $B_{i}$ sets. (2) From each independent $B_{i}$ set, a 7-element feature vector, $x_{i}$ is computed: 3 features that represent HT variance, and 4 features that represent a histogram of HT values. Any $B_{i}$ sets with fewer than $30 \mathrm{HT}$ values were ignored. (3) For each feature vector, $x_{i}$, a single numerical score, $\mathrm{nQi}$, is generated using an ensemble regression approach. Each unit in the ensemble regression includes a linear Support Vector Regression step trained on the Unified Parkinson's disease rating scale part III (UPDRS-III), the clinical score for evaluating PD motor symptoms (Giancardo et al., 2016). . . . . . . . . . . . . . . . . . . . 
4.2 The neuroQWERTY platform. The neuroQWERTY platform was designed to allow for automatic data retrieval of typing data collected at home and remote management by a study coordinator. Operationally, an account in the neuroQWERTY platform was created for each participant in the study. The process included filling in an online form with basic demographics (login information, age and gender), general health information (diagnosed diseases and medication) and some Parkinson's specific data (year of diagnosis, stage of the disease and UPDRS-III). To complete the setup, the data collection software was downloaded and installed in their users' personal laptop to enable remote data collection. Running in the background, the remote monitoring software captured the timing information of any keystroke press/release events, regardless of the application context and content of the typed text. This information, linked to each user account, was encrypted and automatically sent to a remote server through their home internet connection. The neuroQWERTY platform also implemented an administrator module to provide the study coordinators with an interface to control and visualize participants' typing activity . . . . . . . . . . . . . . . .

4.3 At-Home typing activity. Panel A represents the amount of typing data collected from each of the 52 subjects (25 PD, 27 CNT) included in the analysis. The figure highlights the variability in participants' typing routines, as expected from an uncontrolled at-home setting, not only between subjects but also within subjects over time. The red (PD) and blue (CNT) color-scales indicate daily typing activity measured as the number of valid typing windows provided by each subject during the analysis period. We defined a valid window as a sequence of at least 30 keystrokes within 90 seconds of time. To better illustrate the diversity of typing behaviors, panel B illustrates the variability in the amount of typing data with an example from a single PD subject, contrasting the raw typing data corresponding to a low activity day ( 2 valid windows) versus the data collected on a high activity day (60 valid windows). . . . . . . . . . . . . . . .

4.4 nQi at-home algorithm pipeline. The figure represents the pipeline to generate a single neuroQWERTY index (nQi) from a stream of typing data. (1) The typing signal is defined as the time series of hold times (HT) corresponding to each keystroke within a typing routine. This signal is split by non-overlapping 90 seconds windows that the algorithm will evaluate as independent typing units. (2) Only windows with at least 30 keystrokes within the 90-second interval are analyzed. (3) The neuroQWERTY algorithm, previously trained on a separate in-clinic dataset, computes a single numerical score from each independent window. (4) The final nQi is computed as the average of the window-level scores. . . . . . . . . . . . . . .

4.5 nQi in-clinic example. Example of the application of the neuroQWERTY algorithm in an in-clinic typing test. . . . . . . . . . . . . . . 
4.6 nQi iat-home example. The neuroQWERTY algorithm can be used indistinctly to evaluate controlled or natural typing data. This figure represents the at-home typing data and corresponding scores for the same subject shown in Figure 4.5 (note different time scales used in 4.5 and 4.6). Although the uncontrolled activity appears in unpredictable bursts that introduce a high degree of sparsity, our window-based approach allows to analyze the at-home data using a similar method as the one applied for the quasi-continuous in-clinic data. . . . . . . . . . 51

4.7 Comparison of raw typing metrics between In-Clinic and At-Home typing settings. We compared the raw typing metrics measured inclinic and at-home to evaluate how different typing tasks influence the way people interact with the keyboard. The figure shows the correlation of the raw typing metrics, hold time (time between pressing and releasing a key) and flight time (delay between two consecutive key presses), between in-clinic and at-home. Each point represents the metric coordinates (in-clinic, at-home) for each of the 52 participants included in the analysis. Both hold and flight time's values are very similar independently of the typing scenario, as shown by the correlation coefficient values. These results suggest that the in-clinic task does not alter the way subjects type in comparison with their natural typing at-home, which supports our hypothesis that the neuroQWERTY algorithm, built on an in-clinic setting, could be applied to evaluate motor impairment using the typing data from an uncontrolled at-home setting. . . . . . . . . . . . . . .

4.8 Comparison of nQ index (nQi) between In-Clinic and At-Home typing settings. We evaluated the influence of the typing setting in the nQi scores by applying a similar analysis as described in Figure 5 for the raw typing metrics. Panel A shows the correlation of the nQi scores computed in-clinic and at-home. Panel B includes the results of the Bland-Altman analysis to evaluate the agreement of our method in the two typing scenarios. The black line shows the mean difference (d) and the top and bottom dashed lines show the limits of agreement $\left(\mathrm{LoA}, d \pm 1.96 * S D_{d}\right) \ldots \ldots \ldots \ldots \ldots \ldots$ 
4.9 Comparison of nQi performance between In-Clinic and At-Home typing settings. In this figure we compare the classification performance achieved in the two tested scenarios to validate our hypothesis that the neuroQWERTY algorithm, trained on a separate in-clinic dataset, can also be applied to detect PD related motor impairment in an at-home typing setting. Panel A scatterplot illustrates the in-clinic and at-home nQi scores in a patient level. The two black lines represent the classification thresholds computed in-clinic (nQi $=0.0473$ ) and at-home (nQi = 0.0667). These thresholds were estimated for closest-to- $(0,1)$ cut-off points that maximize sensitivity/specificity pairs. Panel B presents the comparison of the receiver operating characteristic (ROC) curves showing the classification rate for the in-clinic and at-home nQi. The plotted curves are the average result of the bootstrapped ROC analysis and the shadowed areas represent the corresponding confidence intervals [5th-95th]. Statistical significance of the Mann-Whitney U test is estimated to reject the null hypothesis that the two groups, PD and CNT, come from the same population. It's noted as: $\mathrm{p}<0.001\left(^{* *}\right), \mathrm{p}<0.01\left(^{* *}\right)$ and $\mathrm{p}<0.05\left(^{*}\right)$. The results show how our in-clinic-built algorithm is able to discriminate PD and CNT from the passively monitored at-home data nearly as well as it was able to discriminate from the controlled in-clinic data. . . . . . . . . . . . . . .

5.1 Schema of study design. The figure presents a schema of the study procedure that comprises a clinical evaluation, finger-tapping test and our typing test. For the clinical evaluation a movement disorder specialist filled-in the motor section in the Unified Parkinson's Rating Scale (UPDRS-III). The alternating finger-tapping test was included as an external reference to quantify upper limbs dexterity. It was performed on a physical keyboard. The typing test consisted of a five minutes task where participants were asked to transcribe a nonstandardized text excerpt using a touchscreen device. The custom screen keyboard and smartphone model used in the test are shown in this figure. . . . . . . . . . . . . . . . . .

5.2 Flight time signals distribution. The figure shows the probability density distribution (PDF) estimated for the normalized flight time (NFT) signals both for each subject (light color) and grouped by condition (dark color). Normalization minimizes the influence of confounding variables related to typing skills. Parkinson's subjects' (PD) distributions present a greater sparsity than controls' (CNT) distributions. A Mann-Whitney $U$ test suggests a significant difference between the NFT values measured on PD participants and controls

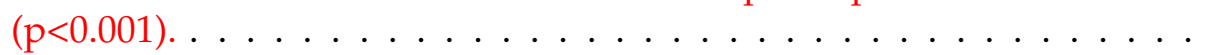


5.3 Feature definition: Graphical examples. The figure compares six signal examples from three people diagnosed with Parkinson's (PD) and three healthy controls (CNT). The normalized flight time series (NFT) is split into 15 second-length windows. Applying Kernel Density Estimation (KDE) we compute the sub-distribution representing the information contained in each window. The mean and standard deviation of the skewness and kurtosis values measured on each subdistribution define four of the features $\left(\overline{S k}, \sigma_{S k}, \bar{K} t, \sigma_{K t}\right)$ that are included in the final 7-dimensional feature vector. The NFT covariance map represents the correlation across the NFT sub-distributions. We define the covariance vector $(\mathrm{Cv})$ as an array including only the coefficients of the covariance matrix corresponding to a valid strictly upper triangular matrix, i.e. above the matrix main diagonal. We extract three metrics from the covariance analysis that complete the typing feature vector $\left(\bar{C} v, \sigma_{C v}, \sum|C v|\right)$. Distributions show a higher uniformity of the NFT values for the CNT's signals compared to PD's. Covariance maps for PD show stronger correlation and anti-correlation within sub-distributions while CNT's maps present values nearer 0 for the entire matrix. . . . . . . . . . . . . .

5.4 PD versus Control, ROC comparison. Comparison of receiver operating characteristic (ROC) curves showing the classification rate for the typing based metrics, including raw flight time average $(\bar{X})$ and best performing univariate $\left(\sum|C v|\right)$ and multivariate methods (Model2), with the alternating finger-tapping test AFT. Statistical significance of the Mann-Whitney $U$ test is estimated to reject the null hypothesis that the two groups, PD and CNT, come from the same population. Statistical significance noted as: $\mathrm{p}<0.001\left(^{* * *}\right), \mathrm{p}<0.01\left(^{* *}\right)$ and $\mathrm{p}<0.05\left(^{*}\right)$.

5.5 Classification performance as a function of typing time. The figure shows the evolution of the area under the ROC curve (AUC) for the best performing feature $\left(\sum|C v|\right)$ and best multivariate model (Model2) as we increment the amount of typing data used to perform the analysis. We observe a clear improvement of the classification performance as the duration of the analyzed typing series increases. . . . . . . . . 70

6.1 Schema of study design. A six-month naturalistic study including a group of 31 early PD patients and 30 matched controls. On the baseline visit, participants where asked to bring their personal laptop to get the neuroQWERTY software installed. The software ran in the background of participants' machine collecting any keystroke data generated during the follow-up period. Over the duration of the study, five on-site evaluations were scheduled. Each visit included a clinical evaluation and an on-site controlled typing task on an standard device. Participants in the PD group were de-novo when recruited for the study. They remained untreated for at least a week from their baseline visit. After that, medication and changes in titration parameters were tracked over the follow-up period. . . . . . . . 75 
6.2 Typing activity map. A representation of the typing activity gathered during the study. The figure presents two heatmaps, left for the PD group and right for the CNT group. Each row in the heatmap provides a sequential representation of each of the participants' typing activity over the 180-day study period. The darker color marks represent the days corresponding to their study visits. The colors in the PD heatmap represent the patients' status with regard to medication response at the end of the study. The blanks represent inactive days. An active typing day was defined by at least 10 valid windows within a day, where a valid window was represented by a data sequence of at least 30 keystrokes in 90-second time interval. To ensure a complete longitudinal representation of the typing patterns over the analysis period, a 15-day rolling window was applied to identify gaps in computer activity. Only participants with one or more active days in at least $80 \%$ of every possible sliding window within the six-month follow-up were considered for the analysis. . . . . . . . . . . . . .

6.3 Feature extraction for At-Home data. This visual represents the data processing designed to define a standard input for the longitudinal at-home typing data. On the first step (A), the keystroke data was grouped by weeks. We used kernel density estimation (KDE) to generate a representation of the distribution of hold times (HT) within that week of data. Additionally, we computed a feature vector as the one described in Chapter 5. For those weeks where not enough typing data was available to compute the analysis, we applied an data imputation method that took the latest available observation to fill the gap. These cases were marked with two additional features: mask, indicating original versus imputed samples, and delta, indicating the timeframe in weeks between the current sample and the data used to fill the gap. . . . . . . . . . . . . . . . . . . .

6.4 nQRNN model architecture. The nQRNN model is designed as a multitask entity. The first task adapts the pre-processing stage from the nQi algorithm to assess the presence or absence of PD motor features in the typing signal. The second task is designed to identify change over time in the longitudinal typing signatures. The results are merged to generate a signal of nQRNNs scores, one per week in the input signal, designed to predict and detect positive response to medication. As for the network architecture, the first task, PD vs CNT, uses fully-connected layers to analyze the feature based data from the at-home typing data. The second task, Change vs No-Change, uses a combination of long-short term memories (LSTM) and fullyconnected layers to reduce the information included in the time series of weekly hold time distributions in the at-home data. The outputs from these two tasks are merged using fully-connected layers. The nQRNN model uses the probability of being identified as a PD responder as the final outcome. . . . . . . . . . . . .

6.5 In-Clinic results: statistical distance metrics. This subplot represents the separation achieved by the proposed statistical distance metrics proposed for the analysis of the in-clinic hold time distributions. The results are presented for both, the prediction scenario (Week 8) and agreement at the end of the follow-up (Week 24). . . . . . . . . . . . . 
6.6 At-Home results: nQRNN model. Similar to Figure 6.5, this graph presents the results for the nQRNN score both, for prediction and agreement with the standard at the end of the study period. Given the longitudinal nature of the data used in this scenario, a representation of the time series of $\mathrm{nQRNN}$ scores computed over time is shown in Panel B. . . . . . . . . . . . . . . . . . . . . . . . . . . . . . 87

6.7 ROC analysis comparison. Comparison of receiver operating characteristic (ROC) curves showing the classification rate for the typing based metrics, the three statistical distance-based metrics (convolution, correlation and Bhattachayyara) the at-home nQRNN. Statistical significance of the Mann-Whitney $U$ test is estimated to reject the null hypothesis that the two groups, PD responders and no-change/nonresponders, come from the same population. Statistical significance noted as: $\left.\mathrm{p}<0.001{ }^{* * *}\right), \mathrm{p}<0.01\left(^{* *}\right)$ and $\left.\mathrm{p}<0.05^{*}\right) \ldots \ldots . \ldots 88$ 


\section{List of Tables}

4.1 Computer Keyboard Study: Dataset Demographics. The table includes a comparison of the clinical and demographic variables between the PD and control groups. The complete study cohort comprised 60 subjects. From the total participants, 52 provided a sufficient amount of at-home typing data (a cumulative total of at least 15 minutes). The table provides a summary of the demographic and clinical information of the participants included in the analysis, 25 patients with PD and 27 control subjects (CNT). The UPDRS-III scale ranges from 0 to 108 (a higher score indicates more severe impairment and disability). For reference, a score of 20 points is typical of patients with very mild disease severity (Martínez-Martín et al., 2015). PD and CNT groups were statistically similar in age and gender, according to a two-sided Mann-Whitney $U$ test. The two groups were also comparable regarding the average daily typing performed during the baseline period. . . . . . . . . . . . . . .

4.2 nQi Performance Comparison. The table compares nQi performance between the in-clinic and at-home typing settings. The classification performance achieved At-Home (0.76 [0.66-0.88] AUC and 0.73/0.69 sensitivity/specificity) is comparable to the results obtained in a controlled In-Clinic setting (0.83 [0.74-0.92] AUC and 0.77/0.72 sensitivity/specificity). The cutoff point was estimated using the closest-to$(0,1)$, i.e. the use case that maximizes the sensitivity/specificity pair (Perkins and Schisterman, 2006). The statistical significance is computed with two-sided Mann-Whitney U test to reject the null hypothesis that PD and CNT subjects come from the same population. The differences between the in-clinic and at-home ROC curves were not significant according to the DeLong test for areas under correlated ROC curves. The method reached a percentage agreement close to $80 \%$ between the two typing scenarios. . . . . . . . . . . . . 54 
5.1 Dataset Demographics. The complete study cohort comprised 51 subjects. From the total participants, 44 provided enough typing information to perform the analysis. A minimum of 5 key presses every 15 seconds during at least half of the duration of the typing task was required to apply the proposed method. Seven subjects, 3 from the Parkinson's group and 4 healthy controls, did not provide enough data and were excluded from the analysis (see Materials and Methods). The table provides a summary of the demographic information of the participants included in the analysis, 21 people diagnosed with Parkinson's (PD) and 23 control subjects (CNT). PD subjects and controls are statistically similar in age, according to the two-sided MannWhitney $U$ test. The same test suggests gender might be a confound variable in this study. These differences were accounted in the analysis. The table also shows the results of the clinical evaluation that includes UPDRS-III, alternating finger-tapping and the Modified Hoehn and Yahr scale. The Hoehn and Yahr scale is a widely used clinical rating scale that defines broad categories of disability in PD in a 0 to 5

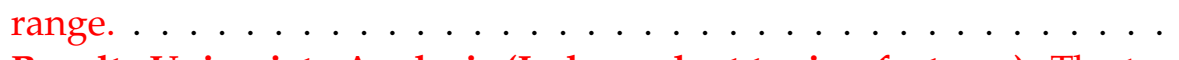

5.2 Results Univariate Analysis (Independent typing features). The table shows the mean values and performance of the typing features and the reference metrics, including the ROC AUC mean and confidence intervals achieved by each measurement and the results of the Mann-Whitney $U$ test to analyze if the null hypothesis, that Parkinson's disease (PD) and control (CNT) subjects come from the same population, can be rejected. Covariance sum $\sum|C v|$ presents the best discrimination performance with an AUC of 0.91 and significance $p<0.001$. 67

5.3 Results Multivariate Analysis (Aggregated typing features). The table summarizes the results of the multivariate analysis. We evaluate the classification performance of different models that aggregate the information of the proposed typing features. We tested a total of nine models, built as the possible combinations of three different feature selection methods and three estimators. A nested cross validation framework was implemented to train and test the models. For each model, we include the mean and confidence intervals of the AUC and the results of the Mann-Whitney $U$ test to reject the null hypothesis that Parkinson's disease (PD) and control (CNT) subjects come from the same population. Model 2, a linear support vector classifier preceded by L1-regularized linear model (Lasso) for feature selection, presents the best discrimination performance with an AUC of 0.88 and significance $\mathrm{p}<0.001 \ldots \ldots$. . . . . . . . . . . 67 
5.4 Methods Comparison. The table compares the performance of the touchscreen typing based metrics, including the raw flight time average $(\bar{X})$ and best performing univariate $\left(\sum|C v|\right)$ and multivariate methods (Model2), with the alternating finger-tapping test AFT. The presented methods improve the discrimination ability of the reference test (AFT: $0.85[0.76,0.94]$ AUC and $0.75 / 0.78$ sensitivity/specificity), with $0.91[0.82,0.97]$ AUC and $0.81 / 0.81$ sensitivity/specificity for the best performing feature $\left(\sum|C v|\right)$ and $0.88[0.78,0.95]$ AUC and $0.73 / 0.84$ sensitivity/specificity for the best multivariate model (Model2). The adequacy of the proposed methods to enhance the differences of the typing patterns between Parkinson's subjects and controls is stressed by the comparison with the raw signal based metric $(\bar{X}: 0.83[0.71$, $0.91]$ AUC and $0.73 / 0.72$ sensitivity/specificity). The presented sensitivity/specificity pairs correspond to the closest-to- $(0,1)$ cut-off point. The unadjusted statistical significance is computed with two-sided Mann-Whitney U test. The adjusted significance tests were computed with logistic regression models including gender and age as co-variates. For the developed methods none of the co-variates reached statistical

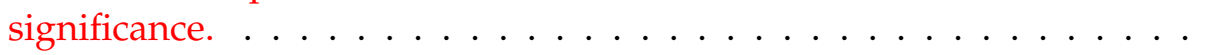

6.1 Demographic and baseline characteristics The table includes a comparison of the clinical and demographic variables between the PD and control groups. . . . . . . . . . . . . . . .

6.2 Metrics performance summary. The table represents the performance achieved by the proposed metrics, both for the ones generated inclinic and at-home, in four different scenarios defined by, inclusion or exclusion of healthy controls in the No-Change group, and time relative to the completion of the study when the scores where evaluated (i.e. prediction vs agreement with response). For each metrics we present the Area Under the ROC curve (AUC), significance defined by the Mann-Whitney $U$, estimated to reject the null hypothesis that the two groups under study come from the same population, and the percentage agreement achieved by the metric under study. . . . . . 



\section{List of Abbreviations}

$\begin{array}{ll}\text { AD } & \text { Alzheimer's Disease } \\ \text { AFT } & \text { Alternating Finger-Tapping } \\ \text { AI } & \text { Artificial Intelligence } \\ \text { AR } & \text { Augmented Reality } \\ \text { AUC } & \text { Area Under the ROC Curve } \\ \text { BG } & \text { Basal Ganglia } \\ \text { CNT } & \text { Healthy Control } \\ \text { COV } & \text { Covariance } \\ \text { DBS } & \text { Deep Brain Stimulation } \\ \text { mPDS } & \text { mobile Parkinson's Disease Score } \\ \text { DSM } & \text { Diagnostic and Statistical Manual of Mental Disorders } \\ \text { DTBZ } & \text { Dihydrotetrabenazine } \\ \text { ECG } & \text { Electrocardiogram } \\ \text { EMG } & \text { Electromyography } \\ \text { FAR } & \text { False Acceptance Rate } \\ \text { FRR } & \text { False Recognition Rate } \\ \text { FT } & \text { Flight Time } \\ \text { HT } & \text { Hold Time } \\ \text { IQR } & \text { Inter Quartile Range } \\ \text { IMU } & \text { Inertial Measurement Units } \\ \text { KDE } & \text { Kernel Density Estimation } \\ \text { LSTM } & \text { Long Short Term Memory } \\ \text { MAO } & \text { Monoamine Oxidase Inhibitors } \\ \text { MCID } & \text { Minimally Clinical Important Change } \\ \text { MDS } & \text { Movement Disorders Society } \\ \text { MIT } & \text { Massachusetts Institute of Technology } \\ \text { MJFF } & \text { Michael J. Fox Foundation } \\ \text { MRI } & \text { Magnetic Resonance Imaging } \\ \text { MTL } & \text { Multi-Task Learning } \\ \text { NFT } & \text { Normalized Flight Time } \\ \text { OMS } & \text { Optoelectronic Measurement Systems } \\ \text { PC } & \text { Personal Computer } \\ \text { PD } & \text { Parkinson's Disease } \\ \text { PDF } & \text { Probability Density Function } \\ \text { PDQ } & \text { Parkinson's Disease Questionnaire } \\ \text { PET } & \text { Positron Emission Tomography } \\ \text { PPMI } & \text { Parkinson's Progression Markers Initiative } \\ \text { nQi } & \text { neuroQWERTY index } \\ \text { nQRNN } & \text { neuroQWERTY Recurrent Neural Network Index } \\ \text { RCT } & \text { Randomized Clinical Trials } \\ \text { RNN } & \text { Recurrent Neural Network } \\ \text { ROC } & \text { Receiver Operating Curve } \\ \text { SD } & \text { Standard Deviation } \\ \end{array}$


xxii

SEM Standard Error of Measurement

SLS Satisfaction with Life Scale

SMA Supplementary Motor Area

SVM Support Vector Machine

SVR Support Vector Regressor

TOM Technology-based Objective Measures

UK United Kingdom

UPDRS Unified Parkinson's Disease Rating Scale

UPDRSIII Unified Parkinson's Disease Rating Scale Part-III

US United States

USA United States of America

VR Virtual Reality 
For/Dedicated to/To my... 



\section{Chapter 1}

\section{Introduction}

The psychomotor domain studies humans' innate ability to develop coordinated motor responses to environmental stimulation (Stolerman and Price, 2011). Through learning and experience, individuals build specific motor mechanisms to appropriately react to sensory information. These motor responses vary from simple instantaneous reflexes to skilled psychomotor functions that integrate cognitive processing of sensory and perceptual information and physical movement. Neurodegeneration causes dysfunction of cognitive, motor and/or sensory abilities, which makes psychomotor impairment a common manifestation of neurodegenerative diseases (Pal and Netravathi, 2005).

Neurodegenerative disorders cover a diverse group of neurological conditions with a variety of etiologies and manifestations, but a common pathophysiology, the dysfunction and ultimate dead of neurons (Bozyczko-Coyne and Williams, 2007). Today, millions of people are affected by neurodegenerative diseases worldwide, and prevalence is expected to increase due to population ageing (Heemels, 2016). Although treatments may provide symptomatic relief for some of the diverse physical or mental manifestations of neurodegenerative disorders, there is currently no cure or therapy to slow disease progression (Stanzione and Tropepi, 2011). Moreover, the lack of specific in-vivo biological tests, together with the subtleness and frequently overlapping clinical signs of these conditions, make accurate diagnosis extremely challenging (Ma, 2010).

Current standards to detect and measure signs of neurodegeneration are limited because of their high rater dependency, variability and episodic nature (Dorsey et al., 2017a). Therefore, decision making relies on sparse and subjective assessments that preclude optimized titration and hinders drug discovery (Palmer et al., 2010). To improve assessments reliability, raters are required to undergo significant training, which limits the number of specialists that can provide an adequate diagnosis and evaluation of the status of the disease. The limited number of expert raters, usually located in specialized centers, increases the cost of clinical evaluations and has a negative impact on patient accessibility and convenience. Moreover, in the context of drug development, variability in current standards reduces the chances of repeatability, which may affect the power of efficacy measures (Brunner, Balc1, and Ludvig, 2012). These challenges highlight the urgent need for more reliable and specific biomarkers to enable earlier diagnosis and better monitoring of disease progression (Noble and Burns, 2010).

The emerging role of digital technologies in healthcare driven by recent advances in information systems, microelectronics, wireless sensors, cloud computing, and data analysis techniques is progressively aligning key industry and research stakeholders in the development and adoption of new digital applications to address major medical needs (Hird, Ghosh, and Kitano, 2016). This healthcare revolution extends to many aspects of management and clinical research in neurodegenerative 
processes, where digital biomarkers are arising as the perfect solution to address the shortcomings of current clinical standards, opening new paths for therapeutic development and personalized medicine (Dorsey et al., 2017a; Beach, 2017). The concepts of mobility and ubiquitous computing are called to disrupt medicine by enabling ubiquitous and unobtrusive automated monitoring and diagnosis, improving traditional health care and boosting the shift towards patient-centered care (Orwat, Graefe, and Faulwasser, 2008; Topol, 2016).

A biomarker can be defined as an objective indicator of the status of a biological process or a measurement of the response to a therapeutic intervention. The label "digital" refers, in this case, to the use of connected digital tools to generate these markers from patient-generated data. The increasing digitalization of large volumes of real world data and recent advancements in technologies to analyze these massive amounts of information are accelerating the introduction of the concept of real world evidence in healthcare. In this new scenario individuals become data producers and technology provides the means to collect, manage and translate these data into meaningful clinical insights (Makady et al., 2017). Since randomized clinical trials, the current standard in drug development, have limitations to provide a complete representation of the actual outcomes of new therapies, real world data is becoming progressively important to help provide broader scientific evidence and a better understanding of new drugs' effectiveness and safety (Sherman et al., 2016). The challenge is particularly tough in Parkinson's Disease (PD) research, where decision making relies on a number of observational measurements thorough the trials, which limits the power of the studies.

Limitations in drug discovery extend to clinical diagnosis and management of PD. Current practices not only lack better monitoring of how PD progresses, but also do not provide a consistent and objective evaluation of the measured signs.The need for an experienced clinician to subjectively evaluate the progress of the disease typically limits the gathering of information to on-site medical examinations (Yang et al., 2016). This clinical data constitutes the main basis on which physicians select and adjust titration parameters, which means that decision-making is subject to patients' recall-bias and is supported by a discrete number of non-representational assessments (Heldman et al., 2014).

The rise of technology adoption is consolidating a highly interconnected world where personal devices are becoming increasingly important in people's daily activities. As we rely more and more on connected devices to complete our routine tasks, we are generating an enormous amount of information from this machine-human interactions. The ability of these devices to automatically collect large volumes of data, together with advancements in computing power, has moved the focus to our capability of explaining of this real-world data. In this scenario, machine learning is growing as our best ally to generate useful insights from this powerful data source. While other industries have made significant progress in the integration of big data and predictive analytics in their business, healthcare is still in its early stages.

In this context, the research proposed in this plan aims to contribute to the acceleration of this digital transformation in healthcare, by leveraging the technological maturity to develop objective biomarkers for psychomotor decline in neurodegenerative diseases based on the automated analysis of patient-generated data. In particular, this work focuses on the introduction and validation of a novel method to quantify psychomotor impairment in PD via natural interaction with electronic devices. 


\subsection{Clinical Context}

PD is the most common movement disorder besides essential tremor and the second most common form of neurodegenerative disease (Tanner and Goldman, 1996). It affects a $0.3 \%$ of the general population (Lau and Breteler, 2006) and $1 \%$ of the population over 60 (Reeve, Simcox, and Turnbull, 2014). Today, there is still no treatment to slow down or even stop the progression of the disease. Available therapy is symptomatic (Jankovic and Aguilar, 2008). Moreover, the incidence and prevalence of PD are expected to increase significantly due to the rapid growth of aging population. Only taking into account the 10 most populous countries in the world, the number of individuals with PD is expected to grow from 4.1 millions in 2005 to 8.7 millions by 2030 (Dorsey et al., 2007). This underscores the importance of further understanding risk factors, advance and develop new therapies and optimize disease management. The development of neuromodifying and neuroprotective therapies that may slow disease progression or even stop neurodegeneration makes early identification and treatment of PD particularly important (Marek et al., 2011). Therefore, investigations of early markers of PD, disease susceptibility and intervention response are critical areas for future research (Miller and O'Callaghan, 2015).

\subsubsection{Pathophysiology and Symptoms}

Neuropathologically, PD is characterized by the loss of nigrostriatal cell and the presence of intracellular $\alpha$-synuclein-positive inclusions known as Lewy bodies (Kordower et al., 2013). From an etiological point of view, both environmental and genetic components have been identified as contributing factors to the onset of the disease (Schapira and Tolosa, 2010). Still, due to the increased vulnerability of dopaminergic neurons, aging continues to be the most strongly related trigger to the pathogenic processes of PD. (Schapira and Jenner, 2011).

The most characteristic biological sign of $\mathrm{PD}$, degeneration of dopaminergic neurons in the substantia nigra, has been traditionally thought as the pathological trigger of the disease. This idea has been put into question by a new theory, introduced by Braak and colleagues (Braak et al., 2003), that suggests a different roadmap for PD pathology with a premotor stage that precedes the symptomatic period. During this phase (stages 1 and 2), Lewy bodies spread from the olfactory bulb and vagus nerve to the lower brainstem, critical areas that explain non-motor symptoms of the disease such as loss of sense of smell, sleep disorders and autonomic symptoms (Schapira, Hartmann, and Agid, 2009). As the disease progresses the transition from premotor to motor disorder starts when the neurodegenerative process moves to the midbrain affecting the substantia nigra (stage 3), mesocortex (stage 4) and neocortex (stages 5 and 6). The loss of dopaminergic neurons, that are responsible for mediating the function of the extrapiyramidal system, disturbs the processing of movement information between the cortex and the striatum (Figure 1.1). These abnormalities lead to the motor symptoms characteristic of PD (Parkinson's disease: diagnosis and clinical management 2008).

The four cardinal signs of the symptomatic period of PD are tremor, bradykinesia, rigidity and postural instability (Jankovic, 2008).

Tremors are the most visible signs of PD, and they are described as involuntary, rhythmic and oscillatory movement that are more prominent in the distal parts of the extremity (i.e. hand or foot). It affects $80-90 \%$ of PD patients (Gelb et al., 1999). The main differential characteristic of PD tremor is that it presents primarily at rest. 
FiguRE 1.1: Braak Stages for Parkinson's pathology. Braak et al. developed a staging system that characterizes disease progression. This system is divided into six different stages, with each stage being attributed to abnormal pathology in particular neurological structures. In terms of symptomatology, the type and severity of symptoms is correlated to progression through the Braak stages. Early stages are characterized by non-motor symptoms. Motor symptoms are often displayed around the mid-stage state, and cognitive symptoms arise as later Braak stages are reached. Modified from (Braak et al., 2003).

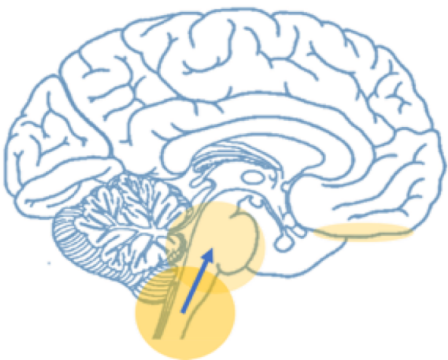

The disease begins in structures of the lower brainstem and the olfactory system. The dorsal motor nucleus of the vagus nerve in the medulla oblongata and anterior olfactory nucleus are affected in Stage 1.

Stage 2 is characterized by additional lesions in the raphe nuclei and gigantocellular reticular nucleus of the medulla oblongata. It then moves up the brainstem, traveling from the medullary structures to the locus ceruleus in the pontine tegmentum.

At the beginning of Stage 3, the disease has entered the substantia nigra and Lewy body lesions begin to form in the pars compacta. Further, structures affected in Stages 1 and 2 begin to develop more Lewy bodies.

Stage 4 is characterized by severe dopaminergic cell destruction in the pars compacta. It also involves mesocortex and allocortex. Pathology can be observed in the amygdala and in the subnuclei of the thalamus. There is significant damage done to the anterior olfactory nucleus.
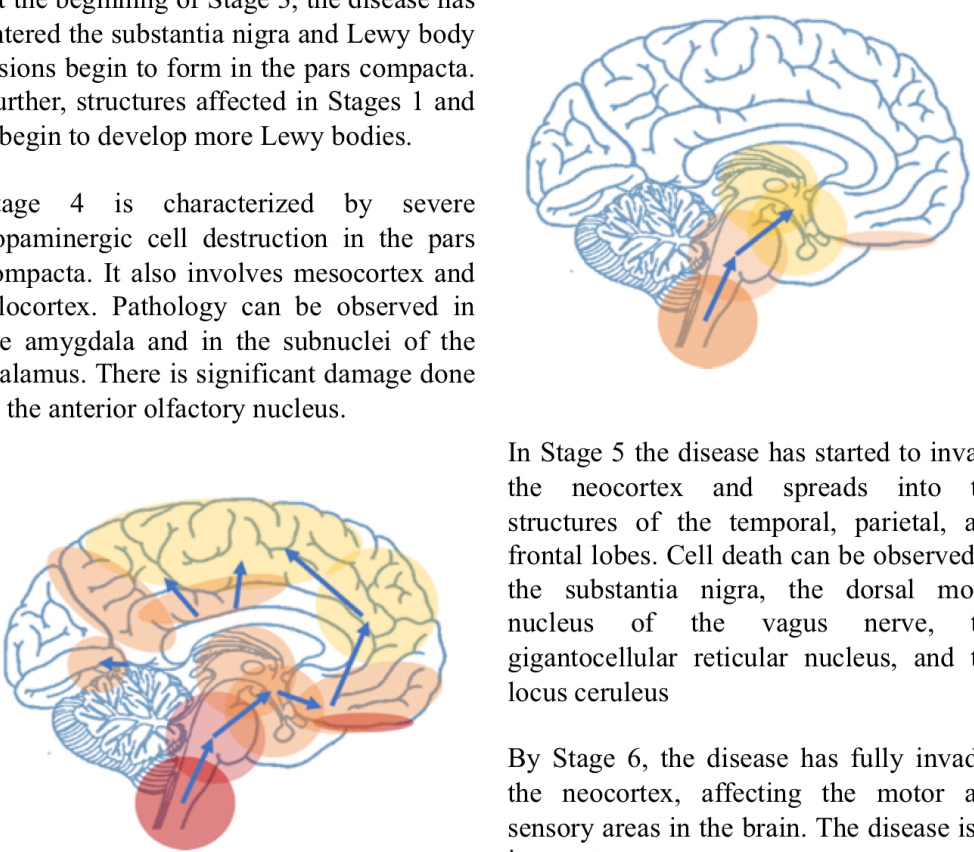

In Stage 5 the disease has started to invade the neocortex and spreads into the structures of the temporal, parietal, and frontal lobes. Cell death can be observed in the substantia nigra, the dorsal motor nucleus of the vagus nerve, the gigantocellular reticular nucleus, and the locus ceruleus

By Stage 6, the disease has fully invaded the neocortex, affecting the motor and sensory areas in the brain. The disease is at its most severe. 
FIGURE 1.2: Parkinson's disease progression and signs. Parkinson's manifests in a variety of symptoms. Among them, four those affecting the motor function are considered the cardinal signs of PD. Specifically, bradykinesia, tremor, rigidity, and postural instability. Even though PD is generally referred as a movement disorder, non-motor symptoms are also common and contribute to disability as the disease progresses. These can include mood disorders and depression, cognitive dysfunction, hyposmia and dysautonomia. Modified from (Poewe et al., 2017).

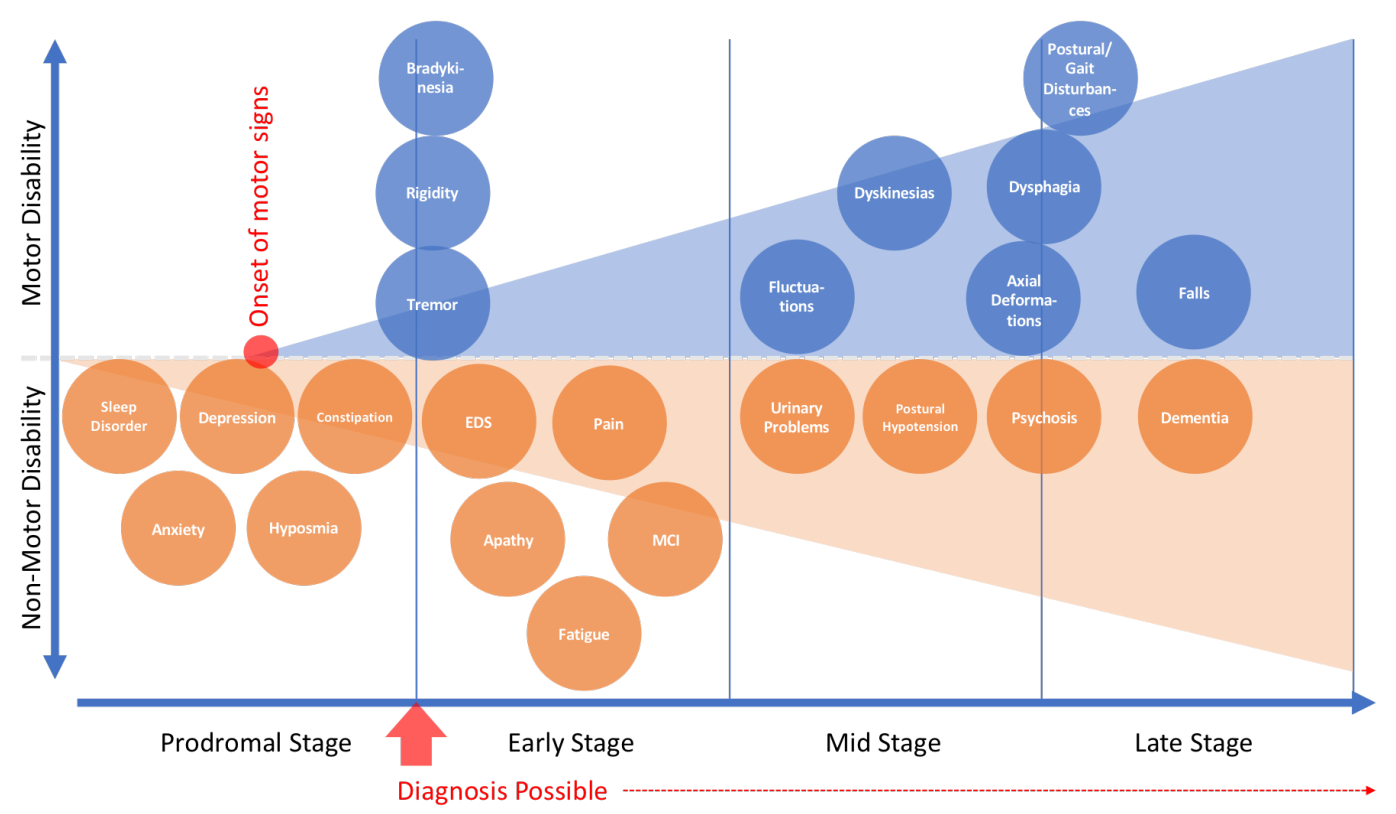

Typically, it disappears with motion or during sleep. In some cases, it can also affect the lips, chin, jaw and legs (Jankovic, 2008).

Bradykinesia is the most characteristic feature of PD. It is defined as slowness of movement that impairs planning, initiation and execution of movement when performing routine activities, sequential and simultaneous tasks (Cooper et al., 1994). Initial manifestations of bradykinesias usually affect tasks requiring fine motor control. This sign has been observed in a 77-98\% of PD patients (Garcia-Borreguero et al., 2002; Gelb et al., 1999).

Rigidity appears as an increased resistance, stiffness or lack of flexibility in the limbs (Grosset, 2009). This involuntary increase in the muscle tone affects $89-99 \%$ of PD patients [68]. It appears as an increased resistance to passive movements of the limb (Gelb et al., 1999).

Postural instability is characteristic of more advanced stages of PD. It appears as a poor balance, unsteadiness, and it is one of the most common cause of falls (GarciaBorreguero et al., 2002). Less frequent than postural instability, but also associated to falls in PD, is freezing of gait or motor block. It is one of the most disabling symptoms of PD and affects $47 \%$ of PD sufferers, (Jankovic, 2008).

Although the motor features described above have been traditionally considered to be the overt clinical signs of PD, there has been a growing awareness to focus on the non-motor features of the disease (Pfeiffer, 2016). The non-motor symptoms of PD include: dementia, depression, psychotic episodes, autonomic dysfunction and oculomotor abnormalities (Gelb et al., 1999) (Figure 1.2). 


\subsubsection{Current Clinical Standard}

The course of PD is chronic and progressive, and affects patients with a complex range of motor and non-motor features, contributing to increased disability and diminishing quality of life (Takahashi et al., 2016).

The symptomatic complexity of the disease manifests in a wide variety of clinical phenotypes (Rooden et al., 2010). This interindividual heterogeneity explains the difficulty in identifying the pathological mechanisms of the disease, which has impacted our understanding of disease progression, prognosis and treatment strategies (Erro et al., 2013).

Current standards to detect and measure signs of neurodegeneration are limited because of their high rater dependency, variability and episodic nature (Dorsey et al., 2017a). Therefore, decision making relies on sparse and subjective assessments that preclude optimized titration and hinders drug discovery (Palmer et al., 2010). In the absence of a specific in-vivo marker of PD, diagnosis is based on the identification of the cardinal signs of the disease. Although repeated assessments tend to improve the accuracy of diagnosis, the limitations of this approach translate into a high rate of misdiagnosed cases (Schrag, Ben-Shlomo, and Quinn, 2002). PD misdiagnosis rate ranges from $10 \%$ to $20 \%$ or greater depending on clinician experience (Hess and Okun, 2016).

The Unified Parkinson's disease Rating Scale (UPDRS) is the most widely used scale to assess impairment and disability in PD and evaluate the adequacy of titration parameters (Ramaker et al., 2002). This scale was specifically designed for its use in PD and provides a comprehensive coverage of motor and non-motor symptoms (Martínez-Martín et al., 1994). The motor examination or UPDRS-III is a subscale that evaluates the motor features of the disease by having the subject undertake a series of tasks to evaluate tremor, bradykinesia, posture, stability and rigidity ("The Unified Parkinson's Disease Rating Scale (UPDRS): Status and recommendations" 2003) (Figure 1.3). The rater assigns a score from 0 to 4 to each of the tasks, being 0 normal performance and 4 completely disabling. The total score for subscale III ranges from 0 to 108, the sum of scores from a total of 27 individual observations. Finger tapping (items 13 and 14) is one of the individual tasks included to assess bradykinesia. The patient is seated and asked to tap the thumb and index finger, making the movements as wide and fast as possible. Item 26 assesses postural stability via the pull test. People with PD tend to have increasing difficulty maintaining balance, particularly with a backwards perturbation (Perlmutter, 2009).

Despite UPDRS-III being the most accepted standard in clinical assessment of $\mathrm{PD}$, it requires significant training to minimize intra-rater and inter-rater variability and it is typically administered by a movement disorder specialist (Taylor Tavares et al., 2005a). Evidence of this variability was presented in a couple of recent studies in PD, where in a phase 2 trial the observed UPDRS change in the placebo group was 6 points in the first one and 8 points in the second study (Kieburtz et al., 2015; NINDS NET-PD Investigators, 2006), even though both shared similar protocols in the same population, sites and raters (Dorsey et al., 2017a). Multiple observations could help reduce the variability introduced by the subjective component of these clinical scales. However, the need for an experienced clinician to evaluate the progress of the disease typically limits the gathering of information to on-site medical examinations. The frequency of on-site evaluations in clinical trials varies from monthly, quarterly to the full duration of the trial (Campenhausen et al., 2011). This medical evaluations are even more infrequent in clinical practice. The episodic nature of these assessments does not provide an accurate representation of the patient's true state 
FIGURE 1.3: MDS-UPDRS Score Sheet The Unified Parkinson's Disease rating scale (UPDRS) is the most commonly used scale in the assessment of PD. The MDS-UPDRS was published by the Movement Disorder Society (MDS) in 2007 as a revision of the original scale. It evaluates various aspects of Parkinson's disease including non-motor and motor experiences of daily living and motor complications. The MDS-UPDRS includes four subscales: Part I (non-motor experiences of daily living), Part II (motor experiences of daily living), Part III (motor examination) and Part IV (motor complications). (Goetz et al., 2008)

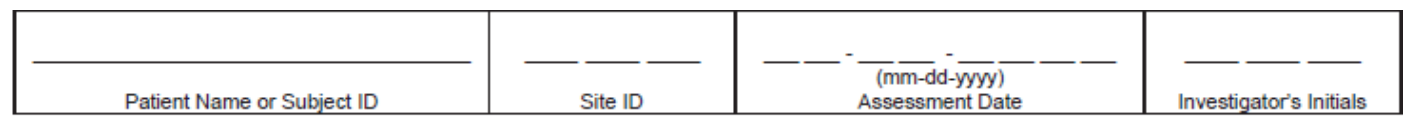

MDS UPDRS Score Sheet

\begin{tabular}{|c|c|c|c|c|c|}
\hline \multirow{2}{*}{$1 . \mathrm{A}$} & \multirow{2}{*}{ Source of information } & \multirow{2}{*}{$\begin{array}{l}\square \text { Patient } \\
\square \text { Caregiver } \\
\square \text { Patient + Caregiver }\end{array}$} & $3.3 b$ & Rigidity- RUE & \\
\hline & & & $3.3 c$ & Rigidity- LUE & \\
\hline \multicolumn{3}{|l|}{ Part I } & $3.3 \mathrm{~d}$ & Rigidity- RLE & \\
\hline 1.1 & Cognitive impairment & & $3.3 e$ & Rigidity- LLE & \\
\hline 1.2 & Hallucinations and psychosis & & $3.4 \mathrm{a}$ & Finger tapping- Right hand & \\
\hline 1.3 & Depressed mood & & $3.4 \mathrm{~b}$ & Finger tapping- Left hand & \\
\hline 1.4 & Anxious mood & & $3.5 a$ & Hand movements- Right hand & \\
\hline 1.5 & Apathy & & $3.5 b$ & Hand movements- Left hand & \\
\hline 1.6 & Features of DDS & & $3.6 a$ & Pronation- supination movements- Right hand & \\
\hline \multirow{2}{*}{$1.6 \mathrm{a}$} & \multirow{2}{*}{ Who is filling out questionnaire } & \multirow{2}{*}{$\begin{array}{l}\square \text { Patient } \\
\square \text { Caregiver } \\
\square \text { Patient + Caregiver }\end{array}$} & $3.6 \mathrm{~b}$ & Pronation- supination movements- Left hand & \\
\hline & & & $3.7 \mathrm{a}$ & Toe tapping-Right foot & \\
\hline 1.7 & Sleep problems & & $3.7 \mathrm{~b}$ & Toe tapping- Left foot & \\
\hline 1.8 & Daytime sleepiness & & $3.8 \mathrm{a}$ & Leg agility- Right leg & \\
\hline 1.9 & Pain and other sensations & & $3.8 \mathrm{~b}$ & Leg agility- Left leg & \\
\hline 1.10 & Urinary problems & & 3.9 & Arising from chair & \\
\hline 1.11 & Constipation problems & & 3.10 & Gait & \\
\hline 1.12 & Light headedness on standing & & 3.11 & Freezing of gait & \\
\hline 1.13 & Fatigue & & 3.12 & Postural stability & \\
\hline \multicolumn{3}{|c|}{ Part II } & 3.13 & Posture & \\
\hline 2.1 & Speech & & 3.14 & Global spontaneity of movement & \\
\hline 2.2 & Saliva and drooling & & $3.15 a$ & Postural tremor- Right hand & \\
\hline 2.3 & Chewing and swallowing & & $3.15 b$ & Postural tremor- Left hand & \\
\hline 2.4 & Eating tasks & & $3.16 \mathrm{a}$ & Kinetic tremor- Right hand & \\
\hline 2.5 & Dressing & & $3.16 \mathrm{~b}$ & Kinetic tremor- Left hand & \\
\hline 2.6 & Hygiene & & $3.17 \mathrm{a}$ & Rest tremor amplitude- RUE & \\
\hline 2.7 & Handwriting & & $3.17 \mathrm{~b}$ & Rest tremor amplitude- LUE & \\
\hline 2.8 & Doing hobbies and other activities & & $3.17 \mathrm{c}$ & Rest tremor amplitude- RLE & \\
\hline 2.9 & Turning in bed & & $3.17 d$ & Rest tremor amplitude- LLE & \\
\hline 2.10 & Tremor & & $3.17 \mathrm{e}$ & Rest tremor amplitude- Lip/jaw & \\
\hline 2.11 & Getting out of bed & & 3.18 & Constancy of rest & \\
\hline 2.12 & Walking and balance & & & Were dyskinesias presen & $\square$ No $\square$ Yes \\
\hline 2.13 & Freezing & & & Did these movements interfere with ratings? & $\square$ No $\square$ Yes \\
\hline 3a & Is the patient on medication? & $\square$ No $\square$ Yes & & Hoehn and Yahr Stage & \\
\hline $3 b$ & Patient's clinical state & $\square$ off $\square$ on & \multicolumn{3}{|c|}{ Part IV } \\
\hline $3 c$ & Is the patient on Levodopa? & $\square_{\text {No }} \square$ Yes & 4.1 & Time spent with dyskinesias & \\
\hline 3.c1 & If yes, minutes since last dose: & & 4.2 & Functional impact of dyskinesias & \\
\hline \multicolumn{3}{|c|}{ Part III } & 4.3 & Time spent in the OFF state & \\
\hline 3.1 & Speech & & 4.4 & Functional impact of fluctuations & \\
\hline 3.2 & Facial expression & & 4.5 & Complexity of motor fluctuations & \\
\hline $3.3 \mathrm{a}$ & Rigidity- Neck & & 4.6 & Painful OFF-state dystonia & \\
\hline
\end{tabular}


(NINDS NET-PD Investigators, 2007). Since in-clinic assessments are carried out at discrete times and in controlled environments, they are not able to capture the fluctuating events of the disease and are subject to observer effect (Torous et al., 2016). Moreover, these physician-based scales have shown varying responsiveness to clinical changes and limited sensitivity to detect disease progression during the prodromal and early stages of PD (Peña-Nogales et al., 2019).

The purely motor assessment of UPDRS-III is complemented by other scales to evaluate other aspects of the disease, including cognitive decline, psychiatric disturbances or autonomic failures. By their nature, these assessments are subjective and highly influenced by patient-recall bias (Papapetropoulos, 2012). These clinical scales constitutes the main basis on decision-making for diagnosis and management of PD.

At this time, there is not known cure for PD. Once the diagnosis is confirmed, the patient will follow a progressive course leading to severe disability and death after a median 7.0 years (Hely et al., 1999) and 15.8 years (Forsaa et al., 2010) respectively. The estimated economic burden of PD in a country like the US exceeds $\$ 14.4$ billion and is projected to increase due to population aging (Kowal et al., 2013).

Unfortunately, there is no therapy that has yet proven to be effective for slowing down the course of the disease, being these therapeutic efforts merely symptomcontrol strategies (Ferreira et al., 2013). The lack of disease-modifier medications motivated several research efforts to develop future drugs with potential to prevent the degenerative process, also known as neuroprotective compounds (Schapira, 2008).

The neuroprotective drugs tested so far in different Parkinson's disease clinical trials have not been successful (Ahlskog and Uitti, 2010). An hypothesis for this failure is that the agents are currently administered too late in the disease (Lang, 2010; Stocchi and Olanow, 2013) course rendering them inactive and this is also based on two main facts:

1. Degeneration of the affected systems takes place, mostly, years before the diagnosis also based on:

- Presynaptic dopaminergic imaging: Studies employing DTBZ-PET (a dopaminergic system tracer) estimated that the degeneration starts 17 years before PD was detected (Fuente-Fernández, 2013).

- Autopsy studies: Quantitative assessments of the cellular density in the nigrostriatal system evidenced at least a $50-90 \%$ neuronal loss by the time the diagnosis was made (Kordower et al., 2013).

2. There is some direct evidence of a greater neuroprotective effect for earlier treatment in both human clinical trials and animal models:

- In the TEMPO study 404 PD patients were randomly assigned to two groups: one started a MAO-B inhibitor (rasagiline) treatment immediately, the control group received the treatment with a delay of 6 months. It was shown that the early treated group had a significantly less worsening in the motor scores compared to the delayed one (Hauser et al., 2009).

- Regarding the experimental evidence that support this second fact, there is a mouse model where the course of the human neurodegenerative process was replicated. The researchers were able to decrease the disease onset on the part of the brain that was treated (Pan-Montojo et al., 2012). 
Current therapies for PD are mainly based on the correction of the nigrostriatal system imbalances with dopamine-containing drugs (Levodopa or agonists). Levodopa is the most effective symptomatic treatment of PD. However, long term levodopa treatment presents several limitations such as "dopa resistant" motor symptoms (postural disabilities, freezing, speech impairment), "dopa resistant" non-motor signs (autonomic dysfunctions, mood and cognitive impairment, etc), or drug related side effects (psychosis, motor fluctuations, and dyskinesias) (Thanvi and Lo, 2004). With further progression of the disease, complications associated to sustained levodopa use can become even more disabling that the disease itself (Block et al., 1997). Optimized titration and adequate dosing strategies are crucial to enhance the outcome of therapy and minimize adverse effects (Brooks, 2008). In some instances, some patients undertake deep brain stimulation (DBS) surgery. This procedure implants thin metal wires in the brain connected to an electrical current source that sends electrical pulses to help control some of the motor symptoms of PD.

\subsection{Statement of Need}

In order to optimize clinical management of PD and maximize the chances of positive outcomes in drug discovery there is an urgent need to find sensitive, specific and reliable biomarkers (Delenclos et al., 2016). Current standards rely too much on clinical observation and hence on the subjective perception of physicians. The wide and complex variability in the disease course, that manifests in a variety of subtypes for each individual case, contributes to the difficulty in defining a standard protocol for diagnosis and treatment of PD. (Lawton et al., 2018). Moreover, the intermittent application of these assessments provides a very restricted representation of the status of the disease and its impact in patients' daily activities.

The introduction of the adequate biomarkers could be crucial, not only to help the scientific community to better understand the underlying pathophysiology of the disease and guide researchers in the development of new therapies, but also to facilitate clinical diagnosis and allow for a more precise way to track disease progression. This would bring us closer to a scenario where it would be possible to provide personalized therapeutic strategies for patients with PD (Titova, Jenner, and Chaudhuri, 2017). Today, the absence of biomarkers for PD not only affects Parkinson's care options but it also a major impediment in the development of neuroprotective and neuromodifying drugs.

\subsubsection{The Impact in Parkinson's Disease}

No definitive biomarkers of Parkinson's have yet been identified or conclusively validated for: (1) Diagnosis to objectively measure the physical characteristics associated with the presence of disease. (2) Disease progression to objectively track changes over time that correlated to PD course.

Specific diagnostic markers could help reduce the rate of misidentified PD cases. Earlier identification of the onset of the disease is critically needed for clinical trials, particularly trials of potential disease-modifying treatments. Today's standards are not able to identify people at risk, which impedes the creation of strategies for PD prevention. A diagnostic marker would allow for earlier identification and intervention in populations at risk. Since the clinical diagnosis of PD usually occurs after a substantial number of dopamine neurons have degenerated (60-70\%), intervention and studies include patients' that are already too advance in the progression of the 
disease (Miller and O'Callaghan, 2015). This may have contributed to a history of inconclusive results from PD trials (Adams and Brantner, 2006). The sole reliance on physicians' perception to identify PD subjects impedes research progress and explains the high number of diagnostic errors early in the disease.

To date, most PD biomarker studies have focused on separating PD patients from healthy controls or from other neurological diseases. While diagnosis confirmation is important, another urgent need is the development of metrics that allow differentiation between subgroups of PD patients that vary in rate of progression along cognitive and motor trajectories (Chen-Plotkin et al., 2018). Progression markers are needed not only to improve current outcomes of PD care, but they could also help transform the development of next-generation PD treatments. Most trials rely on subjective scales, like the UPDRS, to evaluate the efficacy of new treatments. The limitations of these assessments reduce the confidence and replicability of trial findings (Dorsey et al., 2017a). More precise and easy to use tools could provide a more objective evaluation of the impact of new therapies. In the context of PD care, more powerful monitoring markers would enable personalized medicine to adapt titration to each individual's specific disease roadmap.

\subsubsection{Biomarker Characteristics}

According to its formal definition, a biomarker is "a characteristic that is objectively measured and evaluated as an indicator of normal biological processes, pathogenic processes or pharmacological responses to a therapeutic intervention." (Shaw et al., 2007). From a diagnostic point of view, the main characteristics of an ideal biomarker are specificity, sensitivity, predictive value and robustness. In the case of PD, an additional requirement is the ability to discriminate between different subtypes of the disease. In the absence of known specific biological features that identify each of the different Parkinson's subtypes, adequate and precise characterization of the progression of the disease is crucial in order to develop more efficient personalized care approaches (Fereshtehnejad et al., 2017). The complexity of PD pathophysiology impedes the definition of clear metrics to diagnose and track PD processes. Alternative assessments, including measures of motor impairment, disability, quality of life, or disease stage scales, have been used as surrogate biomarkers to understand and evaluate the pathogenic phenomena responsible for PD progression. However, none of the available tools meet the requirements to establish a reliable and standard marker of the disease course. In "A systematic review of biomarkers for disease progression in Parkinson's disease", McGhee et al. concluded:

"The ideal surrogate biomarker should:

1. Change with neurodegeneration (i.e. degeneration of the nigrostriatal dopaminergic system);

2. Show an association with the clinical phenotype arising secondary to this degenerative process;

3. Have a direct association with disease progression, without intermediate variables;

4. Have robust longitudinal data linking it to disease progression;

5. Not be influenced by symptomatic treatment, but only by a true change in the neurodegenerative process; 
6. Predict long-term changes in disease progression by short-term changes in the biomarker;

7. Be generalizable to people with differing characteristics (e.g. age, gender, race);

8. Be continually variable (ideally linearly for simplicity);

9. Be sensitive, reflecting small changes in disease progression;

10. Be quick and cheap to measure, and amenable to blinded assessment;

11. Be suitable for measurement reliably across different centres;

12. Be suitable for repeated measurement in the same patient;

13. Be safe and tolerable to the patient." (McGhee et al., 2013)

Many different biomarkers candidates for PD have been studied in the past. However, none of them meets the criteria required their use in clinical trials (Rascol, 2009). Today, there is no way to obtain to directly measure the number of remaining dopaminergic neurons in vivo. Therefore, clinical rating scales (e.g. UPDRS) have become the only available tool to evaluate the symptomatic effects of putative neuroprotective agents and symptomatic relief medications for PD, which means that the current "gold standard" is subjective, non-linear and captures only a snapshot of the condition (Yang et al., 2016). Consequently, drug development and titration decisions rely on sparse, subjective data, which have led to non effective large-scale drug trials and sub-optimal patient care (Dorsey et al., 2017a).

\subsection{Opportunity and Impact}

This scenario confirms the need to develop an objective long-term measurement of disability in PD patients that provides an objective, accessible and precise assessment of the effects of neurodegeneration. In view of the lack of reliable physiological biomarkers, digital biomarkers may help to address current shortcomings. The evolution of mobile and computer technology has opened the opportunity to develop alternative measurement tools that could satisfy the above requirements (Yang et al., 2016). The combination of technology push from instrument and device developers as well as end-user pull from patients, researchers, and more recently providers, has favored the evolution of the landscape for digital biomarkers (Wright et al., 2017).

It has been estimated that three quarters of the world population have access to mobile communication networks and that more than one third of global smartphone users have installed some type of medical application which will rise to $>50 \%$ by 2018 (Meister, Deiters, and Becker, 2016). The widespread use of connected devices that could host health-related applications is driving this transformation toward an ubiquitous and commoditized health. Innovations in devices, data and machine intelligence are expected to not only provide unprecedented tools to assist patients and physicians in disease and health management, but also improve the drugs performance through improved patient engagement and behavior modifications (Papapetropoulos, Mitsi, and Espay, 2015).

The massive penetration of digital technologies has opened up access to almost limitless volumes of data that can be recorded from multiple digital sources, including users' personal devices, to draw an extended phenotype of patients' status and behaviour (Loi, 2018). The rapid innovation in sensor development and integration 
of these new monitors in connected devices, such as laptops and smartphones, is enabling access to affordable remote monitoring of patients' daily routine and status. The integration of digital data sources in patients' records is allowing clinicians to overcome the restrictions of face-to-face patient care by augmenting the scope and spectrum of clinical assessments and health related information. This amalgam of multi-source data poses serious challenges from a data engineering and analysis perspective. The recent development of advanced machine learning techniques is making it possible to approach this complex problem of extracting meaning from patient data to generate actionable insights and enable decision-making in healthcare (Magoulas and Prentza, 2001). The power of machine learning algorithms to identify patterns from high-dimensional and complex data is being applied in multiple systems and devices in healthcare, including biomarker definition, drug discovery and disease epidemiology (Sun and Reddy, 2013).

In the specific case of $\mathrm{PD}$, researchers have been looking to overcome these difficulties and to establish a more objective assessment of psychomotor decline. Digital solutions have been explored in order to compensate the limitations of the current clinical standard. The need for trained physicians to administer the UPDRS and other motor and cognitive assessments brings some boundaries connected to the subjectivity of this measurements, intra and inter rater inconsistencies and time consuming examinations that can hardly be applied as frequent as it would be necessary to provide an accurate representation of the disease status and progression. The noise introduced by the controlled in-clinic environment where these assessments are conducted also reduces the validity of the clinical judgment, as it might not precisely represent the actual disability experiences by PD patients in their daily lives (Goetz et al., 2008). Currently, the lack of objective, continuous and reliable markers is one of the most demanding difficulties faced with PD (Oung et al., 2015). The development of biomarkers will not only help the scientific community to identify populations at risk, but also facilitate clinical diagnosis. Furthermore, these tools could monitor progression, which could ultimately deliver personalized therapeutic strategies.

\subsubsection{The Opportunity of Early Detection}

The hallmark symptoms of PD, resting tremors, rigidity and postural disabilities, are related to the loss of dopaminergic neurons. All current interventions have limited therapeutic benefit for disease progression because, by the time PD is diagnosed, damage has progressed over an estimated period of 5 to 15 years to a loss of $60 \%$ $80 \%$ of the nigral DA neurons (Miller and O'Callaghan, 2015) (Figure 1.4). While evidences have highlighted the need of detecting this preclinical period (screen subjects at risk of developing PD), the way to address it is still an open question.

As previously mentioned, PD is predominantly a motor disease (Hughes et al., 1992). There are different studies showing that the presence of subtle motor signs alone imply a higher risk of developing the disease. Objective, high-frequency data can guide critical decision-making in therapeutic development and allow for a more efficient evaluation of therapies of increasingly common disorders.

The main global translation of highly sensitive digital biomarkers in the midterm is its use to select individuals at risk of having Parkinson's disease in order to include them in randomized clinical trials (RCT), thus speeding up the testing of new drugs. Currently, there are several key opinion leaders suggesting to start testing neuroprotective agents in people affected with REM-sleep behavior disorder and other at risk populations (Postuma et al., 2012). Although there are not yet RCT including these 
FIGURE 1.4: Clinical representation of Parkinson's Disease. As a chronic progressive disease, PD has a pre-symptomatic interval; that is, a period during which the pathological process has begun, but motor signs required for the clinical diagnosis are absent or not detectable. By the time PD is diagnosed, neurodegeneration is very advanced, which limits the impact of existing drugs to relief the disease symptoms. The ability to identify PD in its preclinical stage may be critical in the development and eventual use of neuroprotective therapies. (Heinzel et al., 2016)



prodromal cases, several agents are being developed that could be tested in these individuals. It is estimated that a new disease-modifying therapy for PD will have a U.S. market potential of $\$ 2$ to $\$ 4$ billion (Sherer et al., 2012).

\subsubsection{The Opportunity of Precise Monitoring}

Many researchers highlight the need for a reliable progression marker of the PD (Marek et al., 2011). As an example, a 55 million US dollar clinical study (PPMI) sponsored by the Michael J. Fox foundation (MJFF) funded by a consortium of 13 industry partners is actively seeking biomarkers that will detect the progression of the prodromal stage of the disease (Haas, Stewart, and Zhang, 2012). The easiness of the technology could facilitate its use as a quantitative aid for the routine management of PD.

The potential of digital technologies to enable detection of subtle changes in motor decline and function across the disease spectrum may also improve the assessment of therapeutic responses. The introduction of novel tools to objectively and continuously track patients' status could enhance the care and safety of patients by promoting aging in place and facilitating the development of precision medicine approaches to therapy.

Digital technology support could provide researchers opportunities to collect real-time, continuous data from people with PD. This information could supplement the details doctors get from patients at appointments and enable researchers to develop more efficient Parkinson's clinical trials. 


\subsection{Objectives}

This research project focuses on the study and implementation of new techniques for diagnosis and assessment of motor performance in people suffering neurodegenerative and movement disorders. Specifically, a new method based on the use of user-device interaction data as main source of information to detect and monitor psychomotor impairment connected to these kind of diseases. The main objective of this work is the development of new methodologies and techniques to quantify motor deterioration to enable early detection of Parkinson's disease and therapy monitoring. The proposed approach relies on the analysis of keystroke dynamics collected from natural finger-keyboard interaction with mechanical and touchscreen devices. The main research tasks will focus on the implementation and evaluation of different algorithms to define biometric indexes connected to users' motor performance.

The work described in this document is part of an ongoing project conducted at the Massachusetts Institute of Technology within the Madrid-MIT M+Vision Consortium. The project, "neuroQWERTY", was created in an effort to to develop tools that can quantify psychomotor signs in people with PD in an objective, accessible and user-friendly way. Success in this work would represent a step towards a completely new paradigm in the management of the disease. Automatic quantification of the disease progression would provide information that is detailed enough to enable tailored treatments, as well as a better understanding of the disease and impact of therapy, favouring the introduction of more efficient drugs.

The fundamental hypothesis underneath this PhD research is that valuable information about individuals' psychomotor health can be derived from the timing data patterns extracted from routine finger-keyboard interactions in computers and smartphones. In particular, it will explore the expression of motor decline in early PD in patients' keystroke patterns. Therefore, this work addresses specific points of the exploration and validation of signal processing and machine learning techniques applied to natural keystroke data to derive objective digital biomarkers of psychomotor impairment in PD. Success in this thesis would represent a step towards a transparent and ubiquitous motor sign assessment method that is objective, convenient, and can produce quasi-continuous ambulatory data on patients' neuromotor performance.

This long-term goal is supported by the following primary objectives to be pursued in this PhD research:

1. At-home validation of mechanical keyboard patterns: Validation of the hypothesis that consistent patterns of early Parkinson's can be derived from the analysis of keystroke data collected from patients' routine use of computers at home.

2. Implementation of smartphone-based keyboard pattern for PD: Development and validation of a new methodology to quantify motor impairment through the analysis of the typing signal collected via smartphone devices.

3. Implementation and design of longitudinal models to measure medication response: Development and evaluation of new models to predict patients' response to dopaminergic therapy through the analysis of at-home typing routines. 


\subsection{Document Organization}

This thesis is structured as follows:

Chapter 2 provides a general overview of existing digital technologies designed to support management of PD. The state of the art reviews solutions based on the following categories: target clinical application, measured clinical sign, enabling technology and level of patient involvement.

Chapter 3 presents the scientific rationale behind the proposed methodology. It evaluates its advantages and key characteristics through the analysis of the four categories introduced in the review of the state of the art. We then introduce the concept of keystroke dynamics and our hypothesis on its connection to PD. Finally, we provide an summary of the proof of concept study presented by the neuroQWERTY team in sleep inertia.

Chapter 4 comprises the first research block in the thesis document. It focuses on the introduction and validation of an algorithm that models mechanical keyboard routines in a remote setting for the detection of early signs of PD.

Chapter 5, the second research block presented in this thesis, introduces a new algorithm that focuses on modeling the effects of early PD through the analysis of touchscreen typing patterns.

Chapter 6 presents the final research block included by this $\mathrm{PhD}$ thesis. It presents a novel approach focused on the characterization of longitudinal typing signals to model medication response in patients with PD.

Chapter 7 contains a summary of the study conclusions and suggestions for future work. 



\section{Chapter 2}

\section{State of the Art}

In view of the rapidly growing demand for systems and devices that can diagnose, monitor and assess response to therapy in PD, biomarker discovery has attracted significant attention in the recent years (Sánchez-Ferro et al., 2016a). Despite the efforts in the clinical community to advance and improve the clinimetric testing results of conventional outcome measures (COMs), digital technologies are opening a new path to overcome the limitations of standard rating scales, questionnaires and clinically-based tests. The irruption of technology-based objective measures (TOMs) is already a reality in clinical trials (Artusi et al., 2018), and it is envisaged that, in the near future, a combination of TOMs and COMs will become the standard in clinical practice (Lim and Tan, 2018).

The sophistication and accessibility of digital technologies are enabling the capture of more and previously inaccessible phenomena in PD, improving the accuracy of clinical assessments (Espay et al., 2016). Although some of these newly introduced TOMs hold promise, none of them gather the characteristic to be validated as an standalone biomarker for PD in clinical practice. The heterogeneity and complexity of PD symptomatology, together with the lack of a robust reference to evaluate and regulate TOM-based endpoints is slowing the integration of these tools in clinical and research practice (Merola et al., 2018). Major opportunities for these tool could arise with their combination into a multidomain and multisensor system that would enable a broader characterization of the motor and non-motor features of PD, improving the sensitivity, accuracy, reproducibility of current standard measures (Espay et al., 2016).

Although there is still a long way in towards the definition of a complete and reliable battery of biomarkers to specifically and accurately detect and quantify disease progression in PD, during the last decade, several TOMs for the assessment of PD motor and non-motor functional disability have been developed with the goal of improving the diagnosis, monitoring and therapeutic landscape in PD (Sánchez-Ferro et al., 2016a). Most of this technologies focus on specific areas of expertise regarding target application, measured clinical feature, enabling technology and patient involvement. The combination of different approaches to each of these categories has defined a heterogeneous catalogue of technical solutions with the potential to bring objectivity, ubiquity and robust granularity to PD management (Del Din et al., 2016).

In this chapter we will review the literature to understand different approaches to each of these four categories in studies employing technology as outcome measures in the assessment of PD-related symptoms, their strengths and weaknesses (Figure 2.1). 
FIGURE 2.1: Digital technologies in Parkinson's disease. In the absence of specific in-vivo biomarkers for diagnosis and disease progression monitoring, PD remains a clinically defined disease. In the last decade, a variety of tecnology-based objective measures (TOMs) have been explored to address this unmet medical need. Given the complexity of the disease, these technologies cover a diverse range of approaches, with different use case focus, symptom targets, enabling technology and level of patient involvement.

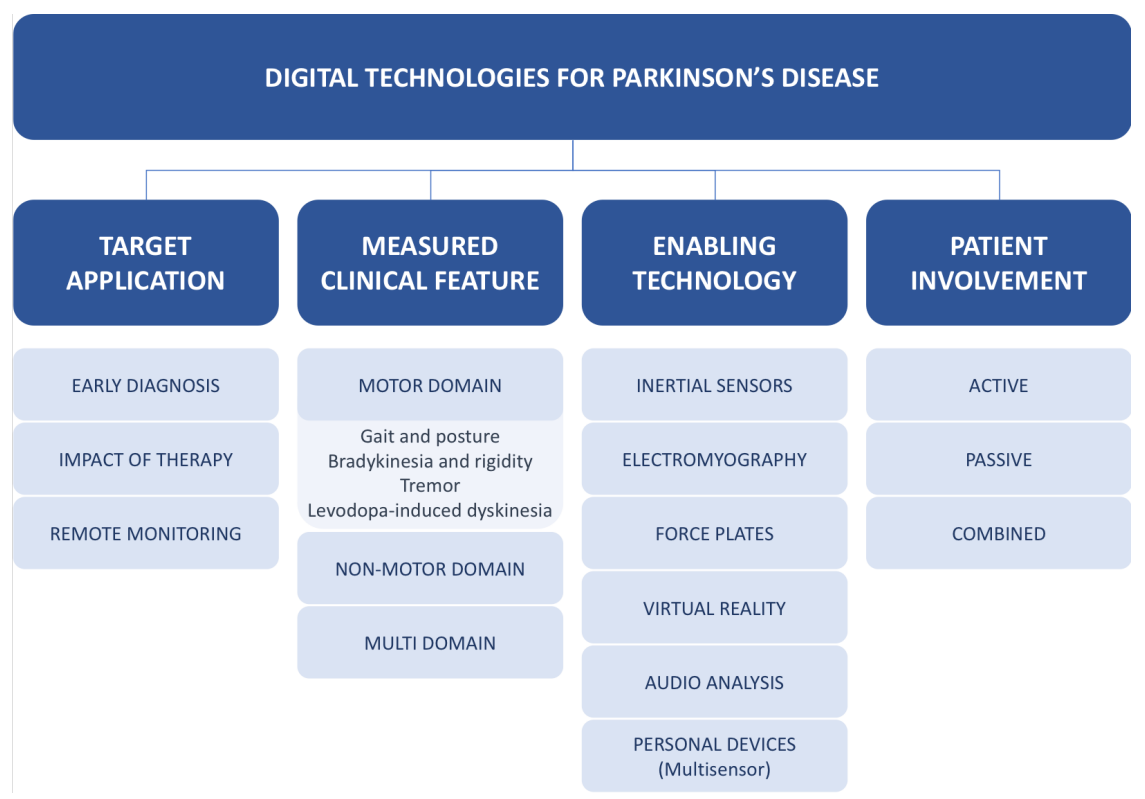

\subsection{Target Application}

The development of an integrated measurement and closed-loop therapeutic system able to support PD patients needs through the different stages in disease diagnosis and progression presents difficult challenges due to the variety of symptoms and the highly individual manifestations of PD. The disease phenotype varies from case to case, as not all people with PD present all the disease signs, and symptom severity may vary between patients and progresses may be at different speeds.

Progression scales, like the modified Hoehn and Yahr (Goetz et al., 2004), have been defined to establish standard progression stages related to the appearance of specific motor and non-motor signs. Therefore, depending on the application target, in the literature we can find different solutions that have been designed to cover the characteristic features of each phase in the pathological context of the application (Rovini, Maremmani, and Cavallo, 2017).

\subsubsection{Early Diagnosis}

On its early stages, PD symptoms can be so mild that patients may not even seek medical attention. Disease onset is usually asymmetrical or unilateral, i.e. symptoms affect one side of the body, and there is usually minimal or not functional impairment (Pagano et al., 2016). Cardinal signs may include intermittent tremor, bradykinesia and rigidity (Rodriguez-Oroz et al., 2009). During this prodromal phase, in which PD signs are present but are insufficient to define a full clinical picture, it is very difficult for physicians to provide a definitive diagnosis (Marsili, Rizzo, and Colosimo, 2018). 
This complexity is also affecting the outcomes of research in the context of technological solutions for early detection of PD (Rovini, Maremmani, and Cavallo, 2017). One of the main challenges in the development of alternative outcome measurements in this space is the need for a large cohort of patients with very mild motor impairment or populations at risk (Sant'Anna, Salarian, and Wickstrom, 2011). Moreover, a confirmed diagnosis based on current clinical standards is necessary in order to validate candidates biomarkers for early diagnosis of the disease, which increases the duration and costs of these research initiatives given that prodromal signs of PD tend to appear over 10 years before clinical diagnosis (Gaenslen et al., 2011).

Some of the existing TOMs exploring early detection of PD focus specifically on the analysis of posture, gait, and asymmetry. Their goal is to objectively identify pathologically abnormal postures characteristic of the prodromal stage of PD (Chen et al., 2014). In their work, Chen et al., evaluated postural sway in rapid eye movement as a potential marker for prodromal PD. Other alternatives for early detection of PD have looked into abnormal expressions of gait, including Brodie et al. (Brodie et al., 2014), that suggested that measurements of reduced movement quality quantified by inertial sensors are linked to early PD. Sant'Anna et al. (Sant'Anna, Salarian, and Wickstrom, 2011) used inertial sensor data to assess movement symmetry focusing on both leg and arm dynamics to separate early PD subjects from healthy controls. Tremor is also a common target in systems that aim to improve specificity in early diagnosis of PD. For example, Perumal and Sankar (Perumal and Sankar, 2016) observed that tremor frequency can be used in early stages of the disease to differentiate PD from other types of parkinsonism.

\subsubsection{Impact of Therapy}

Today, although there is no cure for PD, available medication can be used to provide symptomatic relief. Levodopa (L-dopa) is the most effective drug used to treat PD patients as it provides significant improvement of the symptoms at the beginning of the treatment. Over time, however, the benefits of levodopa frequently diminish or become less consistent. Also, several side-effects are caused by prolonged L-dopa therapy, such as dyskinesias, defined as abnormal, uncontrolled, involuntary movements (Fahn, Group, and Parkinson Study, 2005). Patients in mid and advanced stages of the disease often suffer from motor fluctuations, usually linked to therapy outcomes. Identifying $\mathrm{ON}$ and OFF states in PD is a challenge that difficults management of the disease. OFF episodes appear when the effect of the drug is reduced and symptoms are not controlled. This intensifies the risks of falls, freezing of gait, dyskinesias, tremor and functional impairment (Miyasaki et al., 2002). This state is rarely captured during clinical examinations (Rovini, Maremmani, and Cavallo, 2017). In the literature, we can find several examples of application of TOMs to capture, identify and prevent this critical events.

The use of inertial sensors, such as accelerometers and gyroscopes is a common approach to assess motor fluctuations in the late state of PD and to assess the impact of therapy. Patel et al. (Patel et al., 2009) introduced an accelerometer based system to continiously monitor motor fluctuations in PD by evaluating tremor, bradykinesia and dyskinesia. An algorithm for detection of ON and OFF states in PD patients using a belt-worn single tri-axial accelerometer was presented in (Sama et al., 2012). An alternative based on Electrocardiogram-based (ECG-based) analysis was presented by Ruonala et al. showing an autonomic nervous system response to L-dopa that was measurable through the analysis of heart rate variability (Ruonala et al., 2015). 
Monitoring of adverse effects of L-Dopa therapy is necessary in order to optimize titration parameters and ensure drug benefits (Pérez-López et al., 2016). Interpatient variability in the definition of the threshold between ON and OFF states is one of the main challenges in the validation of existing tools to monitor response to therapy in PD (Tsipouras et al., 2012). This explains why the most accurate results in this specific application context have been found in studies restricted to participants showing a significant change in motor performance between ON and OFF states (Hssayeni, Burack, and Ghoraani, 2016).

\subsubsection{Remote Monitoring}

Current care of PD does not meet the aims of health care quality of safety, effectiveness, patient centeredness, timeliness, efficiency, and equity, as defined by the American Institute of Medicine (Crossing the Quality Chasm 2001). The use of clinical scales as the gold standard for diagnosis and management of PD limits access to proper care, as the requirement for in-clinic assessments makes this care institution based as opposed to the preferred patient-centered (Dorsey et al., 2016). Home-based care could help improve the quality of PD management by enabling remote intervention and self-monitoring to be used in combination with in-clinic assessments for a more personalized treatment (Achey et al., 2014).

Technology appears as the the ideal vehicle to help the medical community to accomplish the goal of home and long-term monitoring for PD patients. Several recent studies have proposed remote monitoring solutions for PD signs. In part, encouraged by grant opportunities and institutional programs, like the REMPARK project, that have emphasized this development closed loop solutions for at-home detection, response and treatment management of PD (Cabestany et al., 2013). Tsipouras et al. presented PERFORM, a multi-parametric system for monitoring and assessment of motor status based on the analysis of multiple signals collected by a multi-sensor wearable unit (Tsipouras et al., 2014). Another example is the method proposed by Ginis et al. in the context of the CuPiD European research project. The system combined inertial sensors and a smartphone-based application to promote gait training in PD patients (Ginis et al., 2016). The lack of a continuous and reliable reference to make sense of the home-collected data is one of the main challenges in the validation of these technologies. Some cases, like the one proposed by Fisher et al., combine passive data collected from wearable technologies with patients' self-reported inputs to provide meaning to the sensor data (Fisher et al., 2016).

\subsection{Measured Clinical Feature}

PD manifests itself in a variety of motor and non-motor symptoms such as bradykinesia, rigidity, and tremor as well as olfactory, impairments, gastrointestinal dysfunction, depression, sleep disturbances, and cognitive impairments (Jankovic, 2008). Intra and inter-patient variability is also characteristic of PD, as it is a highly individual and fluctuating condition. Most patients display a repertoire of motor and non-motor endpoints that change within and between days, with varying impact on their quality of life (Jankovic, 2005). The complexity of disease signs defines the need for integrated technologies able to capture multiple symptom domains in order to provide an accurate picture of the disease status. Thus, multiple sensors and smart technologies are needed to accurately determine the source of all relevant changes, 
characterize individualized disease states, and develop truly personalized therapeutic approaches (Espay et al., 2016).

The variability in disability patterns observed from case to case emphasizes the need for multi-domain systems that are able to adapt to this heterogeneity. For example, upper limb shake measurements may be irrelevant in akinetic cases, where the disease affects primarily postural instability and gait (Zhang et al., 2015). Similarly, analysis of freezing of gait, falls and other severe postural disabilities may not provide any valuable information about PD state and progression during the prodromal and early stages of the disease, as these symptoms tend to appear later when neurodegeneration is more advanced (Okuma, 2014). Solutions that include various data sources that focus on specific aspects of the disease would help identify individuals with different disease phenotypes, understand the correlation between PD cardinal signs and other disability domains, and capture relevant changes in at least one of the integrated data channels (Espay et al., 2016).

While there have been several studies focused on the development of these kind of systems (Tzallas et al., 2014; Samà et al., 2014), the results are still lack the reliability, validation and completeness to be considered appropriate for clinical practice. So far, the study and characterization of the motor signs of the disease has attracted most of the attention in the development of monitoring systems and therapeutic interventions for PD (Pastorino et al., 2013). More recently, the evaluation of nonmotor signs has gained weight in the research community. Still, the knowledge of the pathophysiology of the non-motor domain is limited, and the understanding of how these are connected to disease progression is unclear (Tibar et al., 2018).

\subsubsection{Motor Domain}

PD motor phenotype includes shaking, rigidity, slowness of movement, postural instability and difficulty with walking and gait. As the disease progresses, symptoms become more severe and patients may experience walking difficulties and falls. Furthermore, PD drugs are associated to specific motor complications that make it even harder to assess in an accurate manner the state of motor disability (Opara et al., 2017). The complexity and heterogeneity in the expression of PD-related motor disability is hard to typify using a single measurement tool. For this reason, researchers have mainly focused on the characterization of a reduced subset of the PD motor signs. Most existing approaches to PD motor assessment can be grouped in the following sub-domains: gait and posture, bradykinesia and rigidity, tremor and levodopa-induced dyskinesia (Merola et al., 2018).

\section{Gait and Posture}

Disturbances in posture, balance, and walking commonly appear in advanced stages of the disease. Gait is a particular semi-automatic motor task which is particularly sensitive to fluctuations between Parkinsonian states (Begg and Palaniswami, 2006). Instability episodes result in reduced mobility and increased risk of falls, which diminishes quality of life. Current clinical assessment of these symptoms involves expert observation of patients' posture and gait during a specific motor tasks. These measurements are subjective and, due to its episodic nature, often do not capture the fluctuating severity of gait and posture complications.

Novel methods for locomotion assessment in PD have been explored to open up new channels for objective evaluation posture and gait impairment in PD patients. Current standards applied in the clinic are either too complex and hard to translate 
out of the laboratory, or based on simple outcomes, e.g. stop-watches, that do not provide reliable enough measurements to capture small changes in disease progression (Horak and Mancini, 2013). A common approach in the introduction of new technologies to improve the accuracy and applicability of mobility assessments in PD are on-shoe or insole sensor-based systems (Mariani et al., 2013; Bloem et al., 2016; Klucken et al., 2013). These solutions automatize the evaluation of clinical tests for gait and posture assessment, such as timed up and go or walking trials, using novel spatio-temporal parameters that have been initially validated to discriminate PD ON and OFF states and PD versus control subjects. However, using special footwear and equipment is not always possible and may also affect subject's normal gait (Begg and Palaniswami, 2006), which limits the feasibility of shoe-based wearable systems.

Some alternatives to shoe-embedded sensors that can be found in the literature focus on the analysis of upper and lower limbs bio-mechanics in gait. Mirek et al. used a three-dimensional motion analysis system that showed lower cadence, walking speed and step length in PD patients compare to a matched control group (Mirek, Rudzińska, and Szczudlik, 2007). Lewek et al. found significant differences in arm swing asymmetry between PD patients and controls through the analysis of an optically-based motion capture system (Lewek et al., 2010). Cancela et al. used accelerometry signals from sensors attached to patients limbs and belt to build tailored motor profiles of PD effects in gait and posture (Cancela et al., 2011).

Although important progress has been done in the development of objective quantitative tools for long-term assessment of gait and posture status in PD, most of these efforts have validated systems in a controlled or semi-controlled settings. Some studies have provided some initial validation of these approaches in unsupervised environments (Moro et al., 2010). However, these are limited in terms of sample size, which highlights the need for larger studies to validate the significance of these findings.

\section{Bradykinesia and Rigidity}

Bradykinesia and rigidity are hallmarks for the assessment of PD. Bradykinesia is the most representative motor sign of the disease and it is defined as slowness in the initiation of voluntary movement that progressively reduces the speed and amplitude in repetitive actions (Biase et al., 2018). Clinical evaluations can hardly catch this fatigue of motion speed and amplitude, but it can be captured using instrumented quantitative evaluation. As for rigidity, it manifests as stiffness and resistance to motion, particularly noticeable in joint circular movements. It is the most challenging PD motor sign to measure in an objective way (Gibb and Lees, 1988; Sánchez-Ferro et al., 2016a).

The UPDRS motor scale includes several items focused in the evaluation of these cardinal signs of PD. However, the subtlety of these motor features, specially in early stages of the disease, makes it difficult for movement disorder specialists to provide an accurate diagnosis and assessment of sign severity. To enable a more quantitative evaluation of these motor components, instrumented skill tests have been combined with physician based scales (Opara et al., 2017). One of the most commonly used in upper limb performance assessment is the Purdue Pegboard Test, a manual dexterity task frequently used in rehabilitation services to estimate hand function (Desrosiers et al., 1995). Another example is the Finger-Tapping Test, introduced by Shimoyama et al. in 1990 (Shimoyama, Ninchoji, and Uemura, 1990), where subjects are asked to tap a keyboard button for 15 seconds at each subject's maximum tapping rate. The 
final score is the number of key-taps achieved for the duration of the task. Over the years, variations of the original Finger-Tapping Test have been introduced to refine its sensitivity to PD-specific motor decline. The most accepted and widely used in PD clinical trials is the Alternated Finger-Tapping test (AFT) (Taylor Tavares et al., 2005b).

Regarding advancements in the introduction of digital based systems to complement current clinical standards for bradykinesia and rigidity assessments, the most common technologies are based on the use of inertial measurement units (IMUs), a combination of accelerometers, gyroscopes and magnetometers (Sánchez-Ferro et al., 2016a). A study by Kim et al. introduced a light-weight gyrosensor in the index finger to derive four performance indices that correlated with the clinical assessments of bradykinesia (Kim et al., 2011). Dai et al. used a single IMU evaluate a series of hand grasping actions. The quantitative metrics derived from the statistical analysis of the sensor signals showed better correlation with neurologists' evaluations than other parkinsonian hand performance assessment systems (Dai, Lin, and Lueth, 2015a). In 2013, they presented a more sophisticated system using two force sensor boxes combined with an IMU to evaluate upper limb rigidity (Houde Dai et al., 2013). In the remote assessment end, Roy et al. presented a hybrid system that combined electromyographic and accelerometer data analyzed with neural network algorithms to generate an evolving longitudinal evaluation of motor performance that was able to discriminate normal movement from PD-impaired movement (Roy et al., 2013).

\section{Tremor}

Tremor is the most obvious motor sign of PD. Despite being easily noticeable by physicians, PD-induced tremor can often be confused with other tremor syndromes, such as essential tremor (ET) (Fekete and Jankovic, 2011). ET usually onsets earlier than PD and primarily affects patients during action, while PD tremor occurs more commonly at rest. PD-tremor fluctuations are hard to characterize during episodic on-site evaluations, which can lead to confusion with other diseases. This explains why, by means of sensors, it is the most studied cardinal sign of PD. In the last years there are several studies that have explored the characteristics of PD tremor in order to allow differential diagnosis, or to monitor fluctuations of this symptom (Biase et al., 2018).

Di Biase et al. developed a tremor stability index based on quantitative statistical analysis of triaxial accelerometer tremor recordings taped over the wrist (Biase et al., 2017). The system was able to differentiate PD from ET tremor of the device operator and postural context. Spyers-Ashby et al. proved the reliability of an electromagnetic six-dimensional sensor system for longitudinal tremor measurements (Spyers-Ashby and Stokes, 2000). IMUs have also been used for quantitative analysis of PD tremor. As an example, Dai et al., showed that their IMU-based system was able to provide tremor measurements that highly correlated with a neurologist's exam (Dai, Lin, and Lueth, 2015b).

\section{Levodopa-Induced Dyskinesia}

Levodopa-induced dyskinesia usually appears in PD patients with a history of longterm levodopa treatment. To minimize its effects, a careful history taking and clinical examinations are required to characterize the specific type of dyskinesia in order to optimize titration parameters (Pandey and Srivanitchapoom, 2017). Quantitative 
systems to detect and monitor levodopa-induced dyskinesia have been evaluated in order to provide more details on the characteristic and progression of this symptom to enable better informed titration adjustments.

Mera et al. presented an automated system to capture this effect of medication in PD patients using body-worn motion sensors. A machine learning model was trained to provide a measurement of dyskinesia severity in a scale for 0 to 4 (Mera, Burack, and Giuffrida, 2012). Li et al. used a vision-based deep learning algorithm to generate a computerized index that showed comparable or better responsiveness to dyskinesia than UPDRS (Li et al., 2018). Lee et al. introduced a wearable based algorithm able to overcome the randomness of dyskinetic episodes that presented a significant improvement with regard to other systems that did not account for the fluctuating nature of this motor sign in PD (Lee et al., 2015).

\subsubsection{Non-Motor Domain}

During the past decades, research efforts and disease management have put nonmotor symptoms of PD on a second level (Tibar et al., 2018). Recent studies have suggested that non-motor signs may precede motor symptoms during preclinical stages of PD (Ross et al., 2008; Schenck, Bundlie, and Mahowald, 1996). This has attracted attention from researchers in the development of new methods focused on the detection of these non motor features to define new markers of pre-symptomatic PD stages.

Computer vision systems have been used to quantify facial expression in PD. Facial bradykinesia may cause reduced facial movements that manifests as impairment of emotional expression known as hyponimia (Ricciardi et al., 2016). Bandini et al. used an automated video-based method to study the effects of PD in facial expression. Results showed that control subjects presented greater dynamism during posed an imitated facial expression, being anger and disgust the most affected expressions in participants with PD (Bandini et al., 2017).

Olfactive deficit or hyposmia is another feature linked to PD onset in the last decade. It is present in approximately $90 \%$ of early-stage PD cases, which makes it a reliable preclinical marker of PD (Doty, 2012). Olfactory testing has shown promising results as a complementary diagnostic marker in premotor PD stages (Nielsen et al., 2018; Haehner, Hummel, and Reichmann, 2014; Haehner, Hummel, and Reichmann, 2009). Rovini et al. introduced a machine learning method able to differentiate sensor-based motor phenotypes from healthy subjects, people with idiopathic hyposmia and PD patients (Rovini et al., 2018).

Sleep behavior disorder has also been studied as a target prodromal marker for PD (Postuma et al., 2015). Prashanth et al. combined hyposmia and rapid eye movement sleep behaviour disorder features to develop automatic diagnostic models for PD. Results showed high accuracy and sensitivity in early PD diagnosis (Prashanth et al., 2014).

Non-motor deficits including depression, anxiety and fatigue are among the top sources of disability for PD patients (Espay et al., 2016). However, only a few studies have focused on the analysis of behavioral health to understand the influence of these factors in PD progression. The project Detection of Activity Performance for Health with New Equipment (DAPHNE) is an example of solutions that integrate monitoring the effect of stress and fatigue to provide a wellness score for PD patients (Rovini et al., 2019).

Another popular non-motor characteristic of PD that has been significantly explored in the seek for objective biomarkers for diagnosis and disease management 
is speech. Studies have shown that the prevalence of voice disturbance and volume reduction in PD patients is comparable to other common Parkinsonian features (Ho et al., 1998). The introduction of machine learning has multiplied the power of speech analysis algorithms to define the characteristics of a speech phenotype for PD (Bayestehtashk et al., 2015). Tsanas et al. achieved a 99\% classification accuracy with a speech based algorithm to separate PD from healthy subjects using measures of disphonia as input (Tsanas et al., 2012). Home-based assessment tools using speech to monitor PD severity have also been explored. An example is the method presented by Asgary et al. that combine time, spectral and cepstral annalysis to generate a score that correlated significantly with the UPDRS motor scale (Asgari and Shafran, 2010).

Dementia is a common characteristic of PD and is associated with a more rapid motor and functional decline (Aarsland et al., 2004). Although not many studies have focused on evaluating the rate of cognitive decline in PD, we can find some examples in the literature showing a correlation between PD severity and deficits in the executive function. Driving assessments are one of the approaches that has been used to define predictors of PD outcomes (Worringham et al., 2006). Heikkilä et al. found that variance in on-road driving faults can be linked to levodopa therapy and slowness of visual processing caused by decline of cognitive abilities (Heikkilä et al., 1998). Another example is the method introduced by Klinger et al. that used a virtual reality system to assess cognitive planning deficits in PD (Klinger et al., 2006).

\subsubsection{Multi Domain}

PD encompasses a repertoire of motor and non-motor endpoints that present a significant inter- and intra-patient variability. Given the different levels and types of functional disability in PD, some specific signs may be more representative of one subject's disease stage and progression rate that may not be as important in other cases (Espay et al., 2016). Researchers have tried to account for this variability by creating multidomain, multisensor, intelligent systems able to capture a broader spectrum of PD signs to enable tailored therapeutic approaches.

Heldman et al. evaluated the impact of a sensor-based telehealth diagnostic platform for PD management. The system used a sensor system for motor assessment connected to a tablet-based software that assisted patients through a number of tests to quantify tremor, bradykinesia and dyskinesia. A subset of the study cohort used a telehealth platform instead of the routine on-site appointments. The remote monitored and telehealth group completed the assessments with high compliance and no significant difference were found between the clinical outcomes in the remote managed group and the standard care group (Heldman et al., 2017).

The PERFORM project used a similar approach based on a wearable sensor system that collected accelerometer and gyroscope signals to longitudinally quantify motor deterioration and identify sub-optimal L-dopa dosage parameters. The sensors input was combined with the input from a virtual reality glove that collected information about medication and food intake. Using a series of machine learning classifiers the information was processed to build individual disease profiles to assist physicians in clinical decision making (Tzallas et al., 2014).

In the mPower study, Bot et al. leveraged the opportunity introduced by smartphone technology to develop a multisource data collection app that combined data from patient surveys and periodic sensor-based recordings collected in a series of app-based tasks. The mPower task battery included memory, walking, speech and 
tapping assessments to remotely quantify and identify ON and OFF states in PD (Bot et al., 2016a).

\subsection{Enabling Technology}

In the literature we can find an heterogeneous collection of systems and technologies that have been evaluated as potential instruments to generate quantitative measurements of specific disease domains in PD.

The most frequently used sensor systems according to the literature are based on inertial measurement unit (IMU) technology (Sánchez-Ferro et al., 2016a). IMUs are electronic devices able to measure and report a body's specific force, angular rate, and sometimes the magnetic field surroundings the body, using a combination of accelerometers and gyroscopes, sometimes also magnetometers. In most cases IMUs are used to reconstruct the pose or at least either the position or the orientation of the body they are attached to. An inherent problem of this technology is that its measurements present a high sensitivity to time varying biases and noises, which leads to unreliable longitudinal estimates (Filippeschi et al., 2017). At their current stage of development, IMUs are primarily used to assess bradykinesia (Cancela et al., 2010; Memar et al., 2018), tremors (Giuffrida et al., 2009), and axial features (Ellis et al., 2015).

Before IMUs, most quantitave motion analysis systems for PD were based on the use of optoelectronics. Optoelectronic measurement systems (OMS) detect light signals and use them to estimate the 3D position of a marker via time-of-flight triangulation. Johnels et al. tested a optoelectronic camera system to quantify disturbance in postural, locomotor and manual movement an coordination in patients with PD to quantify the effects of seleginine on the degree of parkinsonism. Another example is the study by Zackrisson et al. that introduced a optoelectronic analysis metrics as a candidate marker for differential diagnosis of PD, multiple system atrophy, and progressive supranuclear palsy (Zackrisson et al., 2013).

Electromyography (EMG), a diagnostic tool that measures electrical activity produced by skeletal muscles, has also shown promise in the definition of quantitative motor features specific of PD. In the literature, most approaches relying on EMG focus on the characterization of pathological tremors, specifically on the objective definition of a PD tremor pattern as opposed to essential tremor (Ruonala et al., 2013; Breit et al., 2008).

Another alternative to motion sensors, traditionally used in the clinic, are force plates. These instruments are used to measure ground-reaction force (Chen et al., 2013). According to Simieli et al., variability in spatiotemporal parameters and kinetics of obstacle avoidance steps can be used to differentiate PD patients from healthy controls. The system combined a optoelectronic analysis component to collect the obstacle parameters and two force plates to capture the kinect metrics (Simieli et al., 2018). Eltoukhy et al. presented a model to characterize kinetic gait abnormalites in PD using a combination of the Microsoft Kinect system and force plates to predict the 3D ground reaction forces during gait (Eltoukhy et al., 2017).

Virtual reality (VR) is also a common component in PD digital assessment methods and rehabilitating methods for PD. Mirelman et al. used treadmill training with virtual obstacles to assess cognitive, motor and functional performance in patients with PD. The results showed significantly improved physical and cognitive performance in patients with PD during complex challenging conditions (Mirelman et al., 2011). Wellness video-game systems, like Wii Fit, were used in a study to assess 
learning, retention and transfer of performance improvements in PD. When compared to a matched healthy control group, patients with PD showed a poorer performance in games associated with cognitive demands that involved decision-making, response inhibition, divided attention and working memory(Mendes et al., 2012). A similar approach was presented by Yen et al. in a study that evaluated the effects of VR-augmented balance training and postural control under different contexts with varying attentional demands (Yen et al., 2011).

PD can affect the volume, tone, rhythm and rate of speech. Audio recording systems have been used to evaluate the impact of the disease in patients' speech and communication (Bayestehtashk et al., 2015; Tsanas et al., 2012; Asgari and Shafran, 2010). Vaiciukynas et al. used two channels to simultaneously record speech signals, an acoustic cardioid and smartphone microphones. The study investigated different audio feature sets from sustained phonation and text-dependent speech modalities for its application in PD screening (Vaiciukynas et al., 2017). Gamboa et al. combined the signals from a microphone and laryngograph equipment to quantify several acoustic parameters of the voice of PD patients. Compared to controls, PD acoustics showed higher jitter, lower harmonic/noise ratio, lower frequency and intensity variability of the sentence, and lower phonational range and presented a higher frequency of the presence of low voice-intensity, monopitch, voice arrests, and struggle (Gamboa et al., 1997).

Researchers in the space have also taken advantage of the opportunities introduced by the widespread use of personal computers and smartphones. Besides its computing ability, these devices incorporate a number of sensors that can be leveraged to capture relevant information about the disease status. A research group from Johns Hopkins University recently introduced the mobile Parkinson's disease score (mDPS), a smartphone-derived rating score to monitor motor impairment severity in a real-world setting (Arora et al., 2015). The app assisted participants to remotely undertake five tasks including voice, finger-tapping, gait, balance and reaction time evaluations. An algorithm aggregated the task performance information to generate a severity score that showed significant correlation with current clinical standards (Zhan et al., 2018). The mPower study is another example of technologies that rely on smartphone technology to longitudinally track PD patients in their natural environment (Bot et al., 2016a). This apps uses the accelerometer, touchscreen and microphone to assess patients' motor and cognitive performance. These data is compared to the information collected in a series of qualitative patient surveys, biological signals and medication intake times. The results showed that the mPower method can be used to quantify severity and fluctuations of PD symptoms (Omberg et al., 2017).

More recently, and in combination with some of the previously mentioned technologies, machine learning has appeared as the common denominator to turn these data into specific clinical outcomes or diagnostic phenotypes. These techniques are able to generate pattern representations from complex data using machine learning models or an ensemble of multiple learning units. Random forest, support vector machines or neural networks are some examples of machine learning algorithm used in the literature to generate novel computational biomarkers to provide clinical decision support for diagnosis and monitoring of PD (Gao et al., 2018). As an example, Abos et al. used a combination of support vector machines and logistic regression to reconstruct patterns of functional connectivity from fMRI to detect cognitive deficits in patients with PD (Abós et al., 2017). Little et al. also used a support vector machine kernel to model speech signals to introduce an improved parametrization method to quantify dysphonia in PD (Little et al., 2009). Advances in machine learning have also enabled the translation of real-world data into real-world evidence from the 
analysis of signals collected from the information generated by PD patients in their daily living. An example is the work presented by Bernad-Elazari et al., a machine learning approach to generate a PD motor phenotype from the analysis of daily transitions in patients with PD (Bernad-Elazari et al., 2016).

\subsection{Patient Involvement}

The way data is collected with regard to the nature of the interaction between patients and technology defines another design variable in the introduction of TOMs for PD. Advances in sensor and network technologies is favoring the integration of clinical data collection with pervasive non-medical infrastructure, augmenting access to real-world data and real-time response.

Up until a few years ago, most TOM's where design based on a series of active tasks to provide a controlled setting for data collection. This approach provide a cleaner context and a more straightforward interpretation of the input signals (Espay et al., 2016). However, this approach is limited in terms of the frequency of information gathering and reduced patient. A recent survey on the state of long term monitoring technologies shows a high dropout rate amongst smartphone apps users: $26 \%$ of apps are used only once and $74 \%$ of apps are not used more than 10 times (Ledger and McCaffrey, 2014). An example of TOM using an active patient or task-based approach is the solution presented Zhan et al. to compute their mDPS score. This app implements a battery of tasks including sustained phonation for speech analysis, standing and walking tasks for posture and gait analysis, and touchscreen-based activities such as finger-tapping or a press-release task to measure reaction time (Zhan et al., 2018).

Patients' lack of motivation to use wearable technology or undertake daily tasks for self-monitoring cannot be underestimated (Espay et al., 2016). To overcome compliance issues and minimize the impact of TOMs in patients' daily living, some researchers have explore the feasibility of approaching data collection in an unobtrusive manner. To date there is no fully validated system to passively monitor clinical features of PD in an at-home setting. However, promising results for some metrics have been reported in the literature (Del Din et al., 2016). Moore et al. implemented a system for long-term monitoring of gait in a natural environment. Data was passively collected using an ankle-mounted sensor that transmitted data to a small pocket PC for analysis. Results showed system was able to capture the small, variable stride length characteristic of Parkinsonian gait, and fluctuations of efficacy associated with levodopa therapy (Moore et al., 2007). Pavel et al. combined machine learning and an in-home unobstrusive sensor network to quantify gait speed and variability. The signals were collected using a set of passive motion detectors and active radio frequency identification tags placed in strategic places within patients' residence and analyzed using hidden Markov models (Pavel et al., 2007). 


\section{Chapter 3}

\section{Scientific Rationale}

Machine learning techniques are opening new paths to solve challenging problems by leveraging real world data. The healthcare community is using these data to provide decision support and improve clinical trial design by combining current standards with the benefits of real-world evidence (Sherman et al., 2016). Based in this novel concept, this thesis aims to introduce and validate new digital biomarkers for PD that rely on the analysis of patients' daily interaction with their electronic devices to objectively, unobtrusively and continuously assess their psychomotor status. Current standards to detect and measure signs of neurodegeneration are limited because of their high rater-dependency, variability and episodic nature (Dorsey et al., 2017a). Therefore, decision making relies on sparse and subjective assessments that preclude optimized titration and hinders drug discovery (Palmer et al., 2010). In the context of drug development, variability of current standards reduces the chances of repeatability, which affects the power of efficacy measures (Brunner, Balc1, and Ludvig, 2012). The proposed approach relies on the use of direct metrics patients' interaction with their personal electronic devices to enable remote and passively collected assessments of disease status in PD management and clinical trials.

Tremor, bradykinesia, dyskinesia or impaired fine coordination are some of the most common motor manifestations of PD, intrinsically linked to disease diagnosis (Hughes et al., 1992; Obeso et al., 2010), and frequently used as endpoints in clinical trials employing patient (Peto, Jenkinson, and Fitzpatrick, 1998; Schuepbach et al., 2013) and physician-based scales (Fahn et al., 2004; Moro et al., 2010), as well as longitudinal technology-based assessments over the course of the trial (Heldman et al., 2014; Maetzler et al., 2013a). The hypothesis underneath this work is that these signs define a specific typing pattern that can be characterized through the analysis of finger-keyboard dynamics.

\subsection{Solution Context and Advantages}

The widespread use of personal electronics has placed typing among the most frequent activities of our daily living. This enables the possibility of leveraging the data from users' natural interaction with their devices to generate useful clinical insights in an unobtrusive manner (Dorsey et al., 2016). The proposed approach addresses the goal of maximizing participants' compliance (Shprecher et al., 2012) as it employs their normal interaction with an electronic device, collecting quasi-continuous motor information that could increase clinical trials efficiency (Holford and Nutt, 2011) and provide a personalized quantitative view of the treatment response at a reasonable cost (Hobart et al., 2007; Lang, 2011). It is noteworthy that, in the US, currently an increasing $80 \%$ of people 65 years or older owns an electronic device and $67 \%$ are using the Internet regularly (Monica Anderson and Andrew Perrin, 2017), thus making this technical approach widely distributable. 
This very second, billions of people are typing, touching, pressing or clicking an electronic device (Marr, 2019). These finger movements are precisely measured and interpreted by devices to action the most diverse functions and features. In this thesis we want to harness fingers interaction on electronics as a biomarker for a massive screening and monitoring tool in the general population. If successful, this has the potential of being a disruptive technology in drug development of psychomotor conditions, thus positively impacting early detection and disease management. The main advantages over any type of existing tool or human expert are its transparency and large scalability. The transparency is linked to the unobtrusiveness of the proposed data collection approach and the possibility of adding this technology to a variety of electronic tools, from mobile phones to coffee machines, without altering their functionality. The scalability is due to the possibility of reaching the vast majority of the population in the industrialized world. Thanks to transparency and scalability, an unparalleled quantity of continuous health data can be collected without any dedicated effort by the patient or clinician, making it possible to address the urgent needs for the management of PD and other psychomotor disorders in the general population. Moreover, the magnitude of the data that can be easily collected paves the way to longitudinal studies in a way that was unimaginable before.

Today, given that the number of movement disorder specialists is limited, expensive to utilize, and frequently located only in specialized centers, efficient assessment and therapeutic development also faces financial and logistic obstacles (Dorsey et al., $2017 \mathrm{~b}$ ). The cost of the technology proposed in this work approaches zero as its embodiment is software only. The use of commodity hardware as biosensors to collect objective data on the psychomotor function, and the application of machine learning to translate these data into clinical outcome measures, introduces a series of unique features and advantages that could help overcome the limitations of current clinical standards.

Evaluating the proposed methodology through the categories introduced in Chapter 2 we can present our approach as follows:

Target Application: The need for a quantitative measure to characterize and assess the effect of neurodegeneration in patients' psychomotor performance applies to any disease stage or event in clinical intervention in PD. Starting with early detection, since current standards rely on the subjective perception of trained physicians and the ability of the the human eye to perceive early signs of motor degeneration is limited. A sub-clinical measurement tool like the one we propose in this thesis has the potential to uncover PD symptoms even before they become visible. This would open the path to test and develop neuroprotective or neuromodifying treatments to slow or even reverse disease progression (Lang, 2011; Streffer et al., 2012). Early therapeutic intervention also requires accurate diagnosis. In PD, misdiagnosis are close to $20-30 \%$ in early stages (Poewe and Wenning, 2002). Atypical parkinsonian disorders including multiple system atrophy, progressive supranuclear palsy, corticobasal degeneration or dementia with Lewy bodies are often identified as PD at stages of the disease when cardinal signs are not obvious (Poewe and Wenning, 2002). Objective characterization of early patterns of the disease could help improve accuracy in differential diagnosis of PD and a better definition of the psychomotor features that should be incorporated in consensus diagnostic criteria.

Another feature that a real-world data based tool could introduce to complement clinical information is the ability to provide continuous assessment of disease 
progression. Current clinical practices for PD management rely on very limited information, which limits physicians ability to optimize medical outcomes. A passive, non-invasive real-time, continuous monitoring solution able to measures progression over time could enable active, predictive and personalized patient management, allowing physicians, patients and caregivers to be more involved in the management of their diseases (Suzuki, Mitoma, and Yoneyama, 2017).

In the intersection between continuous monitoring and precise quantification of psychomotor decline, a digital solution like the one described in this proposal would be of crucial importance as a support outcome measurement for PD clinical trials. The increasing digitalization of large volumes of patient generated data and recent advancements in technologies to analyze this massive amount of information are accelerating the introduction of the concept of real world evidence in healthcare. Since randomized clinical trials, the current standard in drug development, have limitations to provide a complete representation of the actual outcomes of new therapies, real world data is becoming progressively important to help provide broader scientific evidence and a better understanding of new drugs' effectiveness and safety (Dorsey et al., 2017a). As people rely more and more on technology, the information from users' daily interaction with their personal devices appears a promising source of information to enable a more granular representation of psychomotor status in order to better understand and objectively assess the impact of existing and new therapies.

Measured Clinical Feature: The approach introduced in this thesis relies on the hypothesis that a PD phenotype can be derived from the data collected from fingers interaction with electronic devices. Since our finer motor skills are mainly defined by our ability to control the small movements of hands and fingers, actions relying on this skill are the most likely to be affected by damage in the motor cortex (Figure 3.1). The characteristic effects of the disease in fine motor performance may manifest as specific finger movements that could be identified using pattern analysis. According to Jahanshahi et al. (Jahanshahi et al., 1995) one of the initial manifestation of PD is often slowness in performing the normal activities of daily life, especially those tasks requiring fine motor control. This feature, also known as bradykinesia, is particularly present in voluntary motion initiation and sustained repetitive movements, a phenomenon that is expected to have a clear influence in typing dynamics.

Another cardinal feature of PD that may have some effects in the way patients interact with their personal devices is tremor. Although PD tremor is rarely not kinetic, as opposed to essential tremor, rest tremor may impair hand and finger control resulting in difficulty for PD patients when using a keyboard or touching a smartphone screen (Adams, 2017).

Lack of coordination in PD has also been identified as a potential sign of PD. Previous studies have demonstrated the impact of PD in finger interaction and multifinger synergies during finger force production. Specifically, patients with PD present impaired finger individuation, which means a higher force appears in unintended finger pressure (Park et al., 2012). Manual coordination is a key component of finger-device interaction patterns, which suggests this component of impaired feed-forward control could also be reflected from the analysis of this information.

Rigidity in PD sufferers is characterised by increased resistance, present throughout the range of movement of a limb. When accompanied by an underlying tremor it results in a cogwheel phenomenon, which continues throughout an entire range of movement (Jankovic, 2008; Adams, 2017). Finger stiffness is another potential feature of interest in the identification of PD in user-device patterns. 
FIGURE 3.1: Cortical sensory homunculus. A pictorial representation of the anatomical divisions of the primary motor cortex and the primary somatosensory cortex. It represents the portion of the human brain directly responsible for the movement and exchange of sensory and motor information of the body. Areas of the body with more complex and numerous sensory or motor connections are represented as larger in the homunculus. This is the case for hands and fingers, as they control most tasks that require fine motor skills. (Anatomy Eamp;

Physiology)

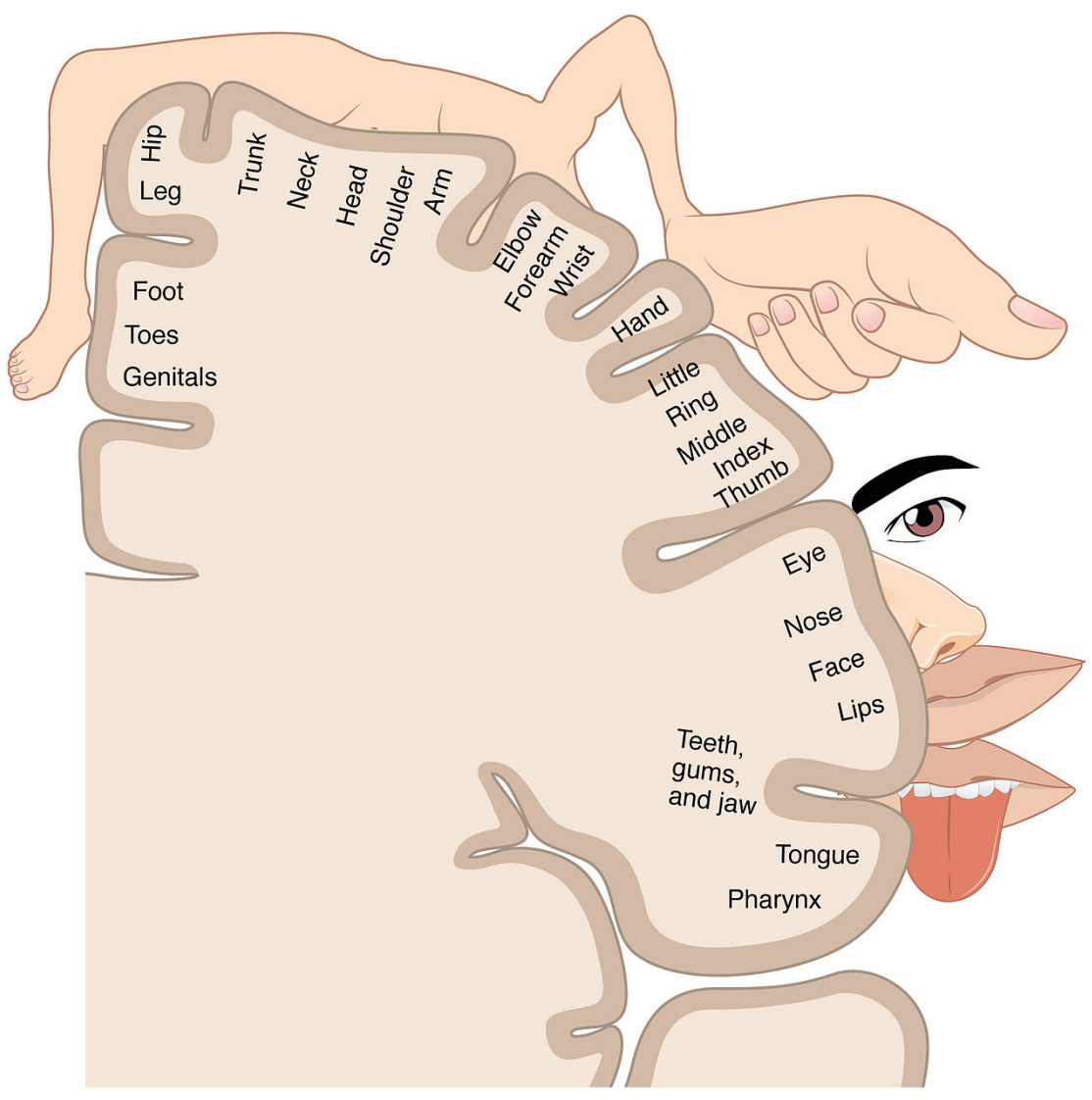


To complete this review of the characteristics of PD motor phenotype that may reflect in the way patients interact with their personal devices, it is worth mentioning the asymmetrical nature of motor impairment, particularly in early stages of the disease (Hoehn and Yahr, 2011). This unilateral decline in motor performance might also reveal some specific features in PD typing signatures.

Non-motor signs of PD, such us cognitive and behavioural dysfunction may also have an influence in patients' daily use of their devices. However, in this thesis, we will only focus on the motor symptomatology.

Enabling Technology: The goal of this thesis is to validate a solution that takes advantage of the pervasiveness and ubiquity of commodity hardware, specifically computers and smartphones. Combining the keystroke data collected from users' natural finger-keyboard interactions with the power of machine learning as a means of identification and measurements of the specific signs of neurodegeneration in PD. The use of keystroke as means of identification has a long history in user authentication (Das, Mukhopadhyay, and Bhattacharya, 2014), but its connection to biometrics research has not been explored until recently (Giancardo et al., 2015). This work will evaluate the influence of PD motor phenotype in keystroke dynamics, gathered from natural interaction with mechanical and touchscreen keyboards, to prove the hypothesis that objective biomarkers of psychomotor decline can be derived via pattern analysis of these data.

Patient Involvement: Most examples found in the literature that explore quantitative tools for remote at-home monitoring of PD signs require patients to perform standardized motor tasks only, as those described in the motor section of the UPDRS (e.g., quiet sitting, finger tapping, finger-to-nose, alternating hand movements, heel tapping, walking) (Patel et al., 2009; Jaywant et al., 2016; Rovini et al., 2018). In this work we focus on the analysis of data collected from one of the most common activities of daily living, as it is users' daily interaction with electronic devices. Compared with other approaches that require preparatory hardware, explicitly launching monitoring apps, and actively undertake a series of standardized tasks generate the measurable input data. This approach aims to enable passive continuous data collection in a patient-friendly measurement tool that also minimizes the potential effect of observer or Hawthorne noise (McCarney et al., 2007a).

\subsection{The Kinematics of Typing}

On May 24, 1844, the message "What hath God wrought" was sent by telegraph from the U.S. Capitol in Washington, D.C. to the Baltimore and Ohio Railroad "outer depot" in Baltimore, Maryland, beginning a new era in long distance communications. By the 1860s the telegraph revolution was in full swing and telegraph operators were a valuable resource (Bryan and Harter, 1897). With experience, each operator developed their unique pattern and was able to be identified simply by their tapping rhythm.

It was soon discovered that operators using this machine, the telegraph, were unwillingly disclosing more information than the message itself. As late as World War II the military transmitted messages through Morse Code. Using a methodology called "The Fist of the Sender", Military Intelligence identified that an individual had a unique way of keying in a message's "dots" and "dashes", creating a rhythm that could help distinguish ally from enemy (Haring, 2007). With the introduction of computer and other keyboard operated devices, this same identification strategy has been evaluated in computer and smartphone devices. The first patent describing a 
method able to identified a person through the analysis of their typing style was introduced in 1983 by J. García ("Personal identification apparatus" 1983). Since then, many studies have proposed novel algorithms to enable keystroke pattern recognition as a biometric tool (Ahmad, Szymkowiak, and Campbell, 2013; Banerjee and Woodard, 2012; Can and Alagoz, 2014). Our hypothesis is that while physical or psychological variations may undermine accuracy for biometric applications, they may be leveraged to infer the psychomotor status of the subject typing (Giancardo et al., 2015).

Keystroke dynamics is a behavioural biometric-based authentication method that identifies the typing rhythm of an individual by observing the timing of keystroke and latency between key events (Al-Obaidi and Al-Jarrah, 2016). The keystroke dynamics method was initially studied on mechanical keyboards, but has recently shifted to the analysis of these data in touchscreen devices (Ali et al., 2017).

With keystroke dynamics the biometric template used to identify an individual is based on the typing pattern, the rhythm and the speed of typing on a keyboard. Keystroke dynamics can be captured via several different features extracted from the typing rhythm of the user including: latency between consecutive keystrokes, flight time, hold time, based on the key down/press/up events, overall typing speed, frequency of errors (use of backspace) and control keys (use of left/right shift). The most commonly used raw measurements used for keystroke dynamics are hold time and flight time. Where hold time is the time duration that a key is pressed and flight time is the time duration in between pressing two consecutive keys. When typing a series of characters, the time the subject needs to find the right key (flight time) and the time he holds down a key (hold time) is specific to that subject, and can be calculated in such a way that it is independent of overall typing speed. Features of keystroke sequences, often used for long texts verification, are typically extracted based on n-graph segments of the entire text. The rhythm with which some sequences of characters are typed can be very person dependent (Figure 3.2). In some cases, we can find the keystroke information collected jointly with other interactions the user has with the computer, such as mouse movements (acceleration time, click frequency) (Moskovitch et al., 2009).

Keystroke dynamics have been more generally used for authentication in conjunction with user ID and password credentials as a form of multifactor authentication. Another use is as a very specific form of surveillance. Some software solutions, often without end-users being aware of it, track keystroke dynamics for each user account in order to generate a history of keystroke dynamics to detect whether an account has been shared and used by other people different from the account owner. Some examples of companies commercializing these kind of products are TypingDNA, that uses AI algorithms to match typing patterns for continuous authentication (TypingDNA - Typing Biometrics, Keystroke Dynamics), and BehavioSec, that specializes on identifying events when the genuine owner of an account is not the main user interacting with the software (BehavioSec: Continuous Authentication Through Behavioral Biometrics).

Although, in general behavioral biometrics such as keystroke dynamics are less reliable than physiological biometrics, they present advantages over other existing approaches. The following criteria summarize the suitability of keystroke dynamics (Keystroke Dynamics):

Universality: This biometric solution can be used by all individuals that are able to use a keyboard.

Uniqueness: Unlike physiological biometric factors, there can be no such thing as an absolute match with behavioral biometrics. Therefore it cannot be the sole 
FIGURE 3.2: A graphical representation of keystroke dynamics variables. Different combinations of timing events of pressing and releasing keys in mechanical or touchscreen keyboards have been traditionally used as a biometric signature to identify an individual based on the manner and the rhythm of their typing. Some of the most common metrics used in this field are the hold time (time between pressing and releasing a key) and the flight time (time between pressing to consecutive keys.)

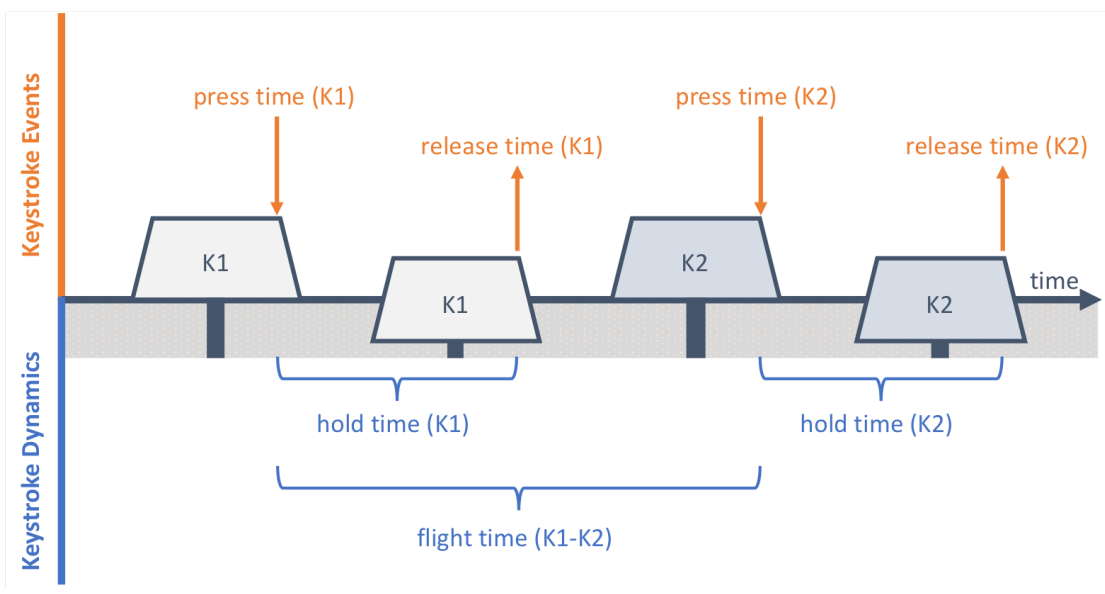

factor to identify or authenticate a subject.

Repeatibility: A major problem with keystroke dynamics is that subjects' typing rhythm varies considerably in between days and even within the same day. There are numerous reasons for this: fatigue, switching devices, changes in mood, influence of alcohol and medications, etc.

Accesibility: An important advantage of keystroke dynamics is that there is no special hardware needed as with other biometrics, a standard computer keyboard is sufficient. It is possible to capture the keyboard dynamics in the background, during longer periods without causing any additional overhead for the subject.

Privacy: Depending of the country or state, using key logging software might be a direct violation of local laws. Even if the actual typed text is not analyzed or retained, applicable legislation must be carefully studied before deploying any software that includes keystroke data collection.

Performance: Behavioral biometrics have higher variations because they depend on a lot of external factors such as ergonomics, fatigue, mood, etc. This causes higher False Acceptance Rate (FAR) and False Recognition Rate (FRR) when compared to solutions based on a physiological biometric factor such as fingerprint recognition.

Current algorithms are highly specialized for biometric identification and are not tuned to characterize health-related variations (Giancardo et al., 2015). The algorithms span a vast range of families: basic statistical features (Joyce and Gupta, 1990; Robinson et al., 1998; Araujo et al., 2005), Bayesian analysis (Bleha, Slivinsky, and Hussien, 1990; Bleha and Obaidat, 1991), autoregressive models (Eltahir et al., 2004), hidden Markov models (Vir V. Phoha et al., 2008), artificial neural networks (Obaidat and Macchiarolo, 1993; Yong, Lai, and Goghill, 2004) and other machine learning techniques (Yu and Cho, 2004).

These solutions have been employed to grant or deny access to a computer system, hence, a primary requirement is the reliance on a small number of key presses in order to avoid excessive burden on the user who needs to access the system (Giancardo et al., 2015). This limits the context in which these algorithms are able to 
recognize user patterns to known predefined text sequences, which significantly improves classification performance. This constitutes one of the main differentiation elements with the approach presented in this thesis. Our aim is to extract relevant psychomotor-related information regardless of the text typed and without changing an individual's daily routine. A recent review (Banerjee and Woodard, 2012) compares various keystrokes dynamics classification methods, some of them achieve an excellent accuracy, with an identification rate higher than $95 \%$. The main challenge that these methods face is the need of taking into account the user variability due to physical or psychological variations. This aspect is consistently reported on most of the studies reviewed. Our hypothesis is that some of these variations might not necessarily be noise, but important physiological variables that could be exploited for medical diagnostics.

The aim of this thesis is to demonstrate that invisible diagnosis of psychomotor symptoms is possible via a computer physical keyboard. Relying on a piece of software that operates in the background of users' personal devices, this tool would be able to monitor typing patterns longitudinally, which can be used to infer or detect changes in health or state, especially considering the number of daily interactions one typically has with keyboards, touchscreen devices or other appliances.

Previous studies applying inverse kinematic analysis of the forces and motion sequence that define users' typing signatures have identified three phases for a single keystroke: key mechanism compression, finger impact, and fingertip pulp compression, followed by release. The key mechanism compression starts when the finger first reaches the contact with the key and ends when the key has been fully pressed, it accounts for $12 \%$ of the total Hold Time (HT). When a key reaches full compression maximum finger deceleration and peak force occurs, this phase accounts for another $11 \%$ of the hold time. Then, the tip of the finger moves down less than a millimeter due to the skin compression and it is finally released from the depressed key, this phase lasts for the remaining $77 \%$ of the hold time. Interestingly, the duration of this phase is not correlated to the forces employed in the first two (Rempel et al., 1994); Jindrich et al. compared the finger tapping kinematics on four structurally different keyboards with three different hand postures, finding that kinematics, endpoint forces, net joint torques, and energy production showed similar patterns (Jindrich, Balakrishnan, and Dennerlein, 2004).

Finger kinematics and motion patterns found from the analysis of users interaction with touchscreen devices are different than those observed in mechanical keyboards (Noah, Li, and Rothrock, 2017). Touchscreen devices allow an heterogeneous number of gestures, each involving different finger-touchscreen dynamics. Overall, there are differences in forces, force direction, and completion times among touchscreen gestures that could be used to estimate musculoskeletal exposure and psychomotor decline (Asakawa et al., 2017; Asakawa, Dennerlein, and Jindrich, 2017).

Previous studies aimed at explaining the neurobiology of typing and keystroke dynamics have revealed that, in mechanical keyboards, hold times are generally very short, typically around 100 milliseconds (Rempel et al., 1994; Kuo et al., 2006); still, keystrokes trigger both cortical and subcortical brain networks which have been identified by neuroimaging functional studies. Witt et al. compared and contrasted 38 independent studies (22 fMRI and 16 PET) solely on finger tapping in order to identify the brain activation areas (Witt, Laird, and Meyerand, 2008). In all studies, the consistent areas activated were: primary sensorimotor cortex (SM1), supplementary motor area (SMA), basal ganglia (BG), and cerebellum. Additionally, clusters of activation were observed in the premotor and parietal cortices, these regions are known to play an important role in the transformation of sensory input to 
motor tasks, and the production of complex motor tasks. Thus, impairment related to these areas may be detectable via changes in patients' typing patterns (Giancardo et al., 2015).

\subsection{The Connection with Parkinson's Disease}

PD-related psychomotor decline is expected to impact the way patients' fingers navigate mechanical and touchscreen keyboards. This thesis support the hypothesis that the fingerprint of the disease in patients daily interactions with their personal devices can be detected and measured via pattern analysis of the keystroke dynamic data. Rigidity, bradykinesia, fluctuations in performance and impaired fine-motor control and coordination may present an specific typing phenotype that may be quantifiable using machine learning analysis. Our aim is to define novel digital biomarkers of fine-motor performance that allow us to translate users' daily behaviour into indices that can be used to support physician's assessments.

Finger enslaving, understood as humans inability to move a finger without moving others is a natural limitation to finger coordination. This phenomenon, addressed as lack of individuation, can has explained by several factors, such as passive connections among fingers, the presence of multitendon extrinsic hand muscles, and overlapping cortical projections (Schieber and Santello, 2004). A healthy amount of enslaving is to some extent optimal for everyday hand function. Nevertheless, increased enslaving should be viewed as detrimental for tasks that require flexible patterns of finger involvement, especially those requiring fine-motor control (e.g. typing). Park et al. have studied the influence of PD neurodegeneration in finger individuation. Their observations suggest changes in supra-spinal control triggered by PD were connected to significantly lower maximal finger forces and higher unintended force production (enslaving) (Park et al., 2012).

Bradykinesia refers to slowness of movement and is the most characteristic clinical feature of PD, although it may also be seen in other disorders, including depression. Bradykinesia is a hallmark of basal ganglia disorders, and it encompasses difficulties with planning, initiating and executing movement and with performing sequential and simultaneous tasks (Jankovic, 2005). Bradykinesia often presents as difficulty with typing, appearing as a decrease in amplitude and frequency of sequential finger movements (Kilgore, 2015). Particularly, it appears as variability and inconsistent tapping movements already reported in previous studies (Jobbágy et al., 2005).

Some specific PD features that, given this characteristics of disease motor phenotype, can be anticipated to appear in the keystroke dynamics patterns are:

1. Slowed reaction times in keystroke sequences. As observed in finger-tapping tests;

2. Prolonged hold times and flight times due to slowness of movement caused by bradykinesia;

3. Asymmetric response in patients presenting laterality of motor signs;

4. Degradation in repetitive movement and typing of sequences of letters due to fine-motor control loss;

5. Mild dyskinesia appearing as abnormal involuntary movements that may interfere with voluntary typing motion; 
FIGURE 3.3: Effect of sleep inertia in typing dynamics. Examples showing the hold time distribution matrices in rested subjects compared with a state of sleep inertia. (Giancardo et al., 2015)

Sample 1



Key Hold Time (ms)
Sample 2

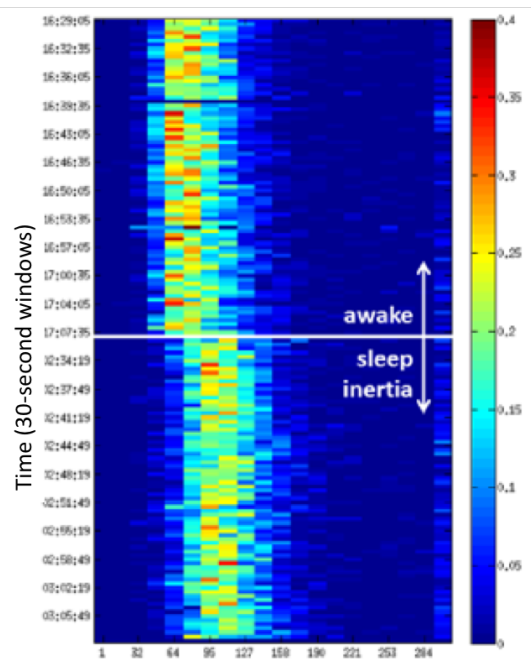

Key Hold Time (ms)

6. Variability and jerkiness of motion caused by tremor and rigidity;

7. Higher rate of typing mistakes caused by lack of finger coordination and cognitive impairment;

8. Marked fluctuations in performance due to ON/OFF states;

9. Long-term performance decline parallel to disease progression.

\subsection{Proof of Concept}

Previous work, published by the research group in which this research plan was developed, has demonstrated that clinically relevant motor function changes can be measured by timing key press/release events from a typing routine in a physical keyboard, irrespective of the language or the typed text (Giancardo et al., 2015).

This work used an induced psychomotor, sleep inertia, as proof of principle. Sleep inertia has been described as a state of grogginess, impaired cognition, reduced motor dexterity, and disorientation while awakening (Scheer et al., 2008). Giancardo et al. presented a novel algorithm that evaluated the evolution of hold time sequences to detect the sleep inertia-induced motor decline in a group of healthy subjects (Figure 3.3).

The team built a browser plugin able to continuously and transparently track the timestamps of key presses and releases in a personal computer. The user can perform her routine task on the browser such as internet browsing, document editing, data entry, while the plugin captures the precise keyboard dynamics data. A set of numerical features to derived from the hold time signals were used to train a machine learning model that presented an AUC of 0.93/0.91 and repeatibility of the proposed approach in two different time points. These results suggest that a system to detect psychomotor impairment is feasible, sufficiently accurate and reliable via routine typing in a relatively unconstrained setting (Giancardo et al., 2015). 
This opens the path to the evaluation of a similar approach to support detection and management of neurodegeneration in diseases such us PD. The proposed method addresses the goal of maximizing participants' compliance as it relies on real-world data (user-device natural interaction) to passively monitor their psychomotor status. This quasi-continuous patient-centered tool could provide a personalized quantitative view of the intervention response and increase the clinical trial efficiency by:

1. Filling the gaps between in-clinic visits and providing more continuous information, reducing random error and boosting statistical power and efficiency of clinical trials;

2. Having more quantitative metrics that remove the subjective component of some of the existing endpoints;

3. Asymmetric response in patients presenting laterality of motor signs;

4. Providing a more granular and precise metric for earlier detection of therapy outcomes, which could reduce the duration and cost of the trial;

5. Helping in selecting candidates for disease modifying strategies by identifying subtle motor changes during the pre-motor phase of the disease. 



\section{Chapter 4}

\section{Keyboard Typing as an Indicator of Early Parkinson's Disease}

Current medical practice in PD has limited tools to routinely monitor motor signs with enough frequency and without undue burden for patients and the healthcare system. In this chapter, we present data indicating that the routine interaction with computer keyboards can be used to detect motor signs in the early stages of PD. We explore a solution that measures the key hold times (the time required to press and release a key) during the normal use of a computer without any change in hardware and converts it to a PD motor index. This is achieved by the automatic discovery of patterns in the time series of key hold times using an ensemble regression algorithm. The result is the neuroQWERTY index (nQi), a new digital marker able to detect motor impairment in an early PD cohort through the analysis of the key press/release timing data collected during both during a controlled in-clinic typing task, and in a free typing at-home setting. The finding that an algorithm trained on data from an in-clinic setting has comparable performance with tested on data collected through naturalistic at home computer use reinforces the hypothesis that subtle differences in motor function can be detected from typing behavior. This work represents another step towards an objective, user-convenient and quasi-continuous monitoring tool for PD.

This chapter comprises the results published in "Computer keyboard interaction as an indicator of early Parkinson's disease" (Giancardo et al., 2016), where we introduced the nQi model, and demonstrated its ability to distinguish PD patients at the early stage of the disease from comparable healthy controls from the analysis of simulated natural interactions with standard keyboards in a controlled on-site typing task, and "Detecting Motor Impairment in Early Parkinson's Disease via Natural Typing Interaction With Keyboards: Validation of the neuroQWERTY Approach in an Uncontrolled At-Home Setting" (Arroyo-Gallego et al., 2018b), where we presented the results of the validation of our in-clinic built algorithm to detect PD typing patterns in an uncontrolled real-world setting.

\subsection{Methods}

The results presented in this work analyze the baseline data collected as part of a 6-month longitudinal PD drug-responsiveness study (NCT02522065). All the experimental protocols were approved by the Massachusetts Institute of Technology, USA (Committee on the Use of Humans as Experimental Subjects approval no. 1412006804), HM Hospitales, Spain (no. 15.05.796-GHM), Hospital 12 de Octubre, Spain (no. CEIC:14/090) and Hospital Clínico San Carlos, Spain (no.14/136-E). All subjects 
provided informed consent prior to study enrollment. The recruitment and experimental procedures were carried out following the relevant institutional guidelines.

The study cohort consisted of 60 subjects, 30 people with recently-diagnosed Parkinson's (PD) and 30 healthy controls (CNT). Only subjects that self-reported at least 30 minutes of daily laptop use were considered for the study. Exclusion criteria included cognitive impairment, upper limb functional limitation, sleep disorders and use of antipsychotics/sedative drugs. At the moment of enrollment, six PD were on rasagiline while the remaining 24 were completely drug naïve. Notably, unlike levodopa or dopamine agonists, rasagiline is a compound that has a little impact on motor performance so, for the purposes of this study related to motor performance, we considered patients on rasagiline to be similar to the PD who hadn't yet started medication. They maintained their baseline medication status (i.e. no drug or continued rasagiline) for a period of time after the enrollment visit.

Enrolled participants underwent an initial baseline assessment in the clinic that included clinical evaluation, an in-clinic controlled typing test, and the technical setup to enable at-home monitoring. The medical examination included an UPDRSIIIbased evaluation carried out by movement disorder specialists. For the in-clinic typing test participants were asked to transcribe a non-standardized sample text on a standard word processor during 15 minutes. To emulate natural interaction with the device, subjects were asked to type as they normally would do at home. A standard machine was used in the in-clinic setting, specifically, a Lenovo G50-70 i3-4005U with 4GB of memory and a 15 inches screen running Manjaro Linux operative system. While undertaking the test, the data collection software ran in the background. Once the task was completed, the typing data was sent to our database server. As part of the baseline visit workflow, the data collection software was installed on participants' personal laptop to enable at-home remote monitoring. If they shared their computer, we provided them with a laptop with pre-installed software. Subjects were encouraged to enter into the routine of typing an email or a document for at least 15 minutes per day but otherwise use the computer as they would do normally.

Once enrolled in the study, PD subjects kept their baseline medication status for about a week. This baseline period allowed an unbiased comparison between the in-clinic and at-home conditions on the assessment of our method. Because of the naturalistic design, there was some variability in the time between initial visit and the date the new therapy was started (i.e. some variability in the duration of the baseline period). This period ranged from 0 to 63 days. For the data reported here, we used a 7-day baseline period, unless there was a medication change within that timeframe, in which case we used the actual baseline period. For the control group, the baseline period was defined as the 7-day period since the date they first logged in to the neuroQWERTY platform.

To assure a comparable amount of typing activity between the in-clinic and athome settings, only subjects that aggregated at least 15 minutes of typing data during their corresponding at-home baseline period were included in the analysis. Though, importantly, data at home was sparsely distributed over the multi-day baseline period, while the in clinic data was concentrated in a 15-minute continuous typing task. To manage this sparsity in the at-home data, we applied the concept of valid window to filter typing gaps and low activity intervals. A valid window was defined as a data sequence of at least 30 keystrokes within 90-second time interval. Parameters related to window sizes and minimum number of keystrokes per window were empirically defined. We strived to identify the shortest possible time window that was likely to contain enough data to estimate the HT probability density function. Five PD and three CNT subjects were excluded from the analysis because they did not 
TABLE 4.1: Computer Keyboard Study: Dataset Demographics. The table includes a comparison of the clinical and demographic variables between the PD and control groups. The complete study cohort comprised 60 subjects. From the total participants, 52 provided a sufficient amount of at-home typing data (a cumulative total of at least 15 minutes). The table provides a summary of the demographic and clinical information of the participants included in the analysis, 25 patients with PD and 27 control subjects (CNT). The UPDRS-III scale ranges from 0 to 108 (a higher score indicates more severe impairment and disability). For reference, a score of 20 points is typical of patients with very mild disease severity (Martínez-Martín et al., 2015). PD and CNT groups were statistically similar in age and gender, according to a two-sided Mann-Whitney $U$ test. The two groups were also comparable regarding the average daily typing performed during the baseline period.

\begin{tabular}{lccc}
\hline Variable & Parkinson's $(\mathrm{n}=25)$ & Healthy controls $(\mathrm{n}=27)$ & $\mathrm{P}$-value \\
\hline \hline UPDRS-III, mean (SD) & $20.48(6.56)$ & $1.93(1.84)$ & $\mathrm{p}<0.001$ \\
\hline Age in years, mean (SD) & $60.2(12.0)$ & $60.81(10.63)$ & $\mathrm{p}=0.73$ \\
\hline Number of women, $\mathrm{n}(\%)$ & $12(48)$ & $14(52)$ & $\mathrm{p}=0.73$ \\
\hline Number of men, $\mathrm{n}(\%)$ & $13(52)$ & $13(48)$ & $\mathrm{p}=0.73$ \\
\hline Daily typing in minutes, mean (SD) & $24.58(15.91)$ & $23.58(14.68)$ & $\mathrm{p}=0.61$ \\
\hline \hline
\end{tabular}

reach the equivalent 15 -minute active typing threshold (10 valid windows) during the baseline period.

A summary of demographic and clinical information for the resulting cohort, 25 PD and 27 CNT, can be found in Table 4.1. Regarding PD severity, all PD subjects were newly diagnosed cases and in the very early stages of the disease, with an average UPDRS-III score of 20.48 points. For reference, a score of 20 points is typical of patients with very mild disease severity (Martínez-Martín et al., 2015). The two groups were matched in age, gender and volume of daily typing.

\subsubsection{The neuroQWERTY index (nQi)}

The nQi is the output of a computational algorithm that uses the information contained in the sequences of hold times, the time between pressing and releasing each key on a mechanical keyboard, to detect evidence of PD motor impairment. First, we introduce a new type of typing signal representation extending our initial work (Giancardo et al., 2016) with variance analysis features; then, we use an ensemble approach based on linear $\varepsilon$-Support Vector Regression to generate nQi scores. Figure 4.1 shows a visual representation of the algorithmic pipeline.

\section{Signal Representation}

Let the vector $a[t]$ represent continuous-time stochastic process of key hold times where $t$ is the time at which each key has been pressed. We consider only the keys for which we expect a short hold time, i.e. alphanumeric characters, symbols and space bar. We define a square window $\omega$, such that:

$$
\omega[n]= \begin{cases}1, & \text { if } 0 \leq n<N^{w} \\ 0, & \text { otherwise }\end{cases}
$$


FIGURE 4.1: Pipeline of the algorithm to generate the neuroQWERTY score (nQi) from the hold time (HT) series. (1) The HT time events are split by non overlapping 90 -seconds windows to create the $B_{i}$ sets. (2) From each independent $B_{i}$ set, a 7-element feature vector, $x_{i}$ is computed: 3 features that represent HT variance, and 4 features that represent a histogram of HT values. Any $B_{i}$ sets with fewer than 30 HT values were ignored. (3) For each feature vector, $x_{i}$, a single numerical score, $\mathrm{nQi}$, is generated using an ensemble regression approach. Each unit in the ensemble regression includes a linear Support Vector Regression step trained on the Unified Parkinson's disease rating scale part III (UPDRS-III), the clinical score for evaluating PD motor symptoms (Giancardo et al., 2016).

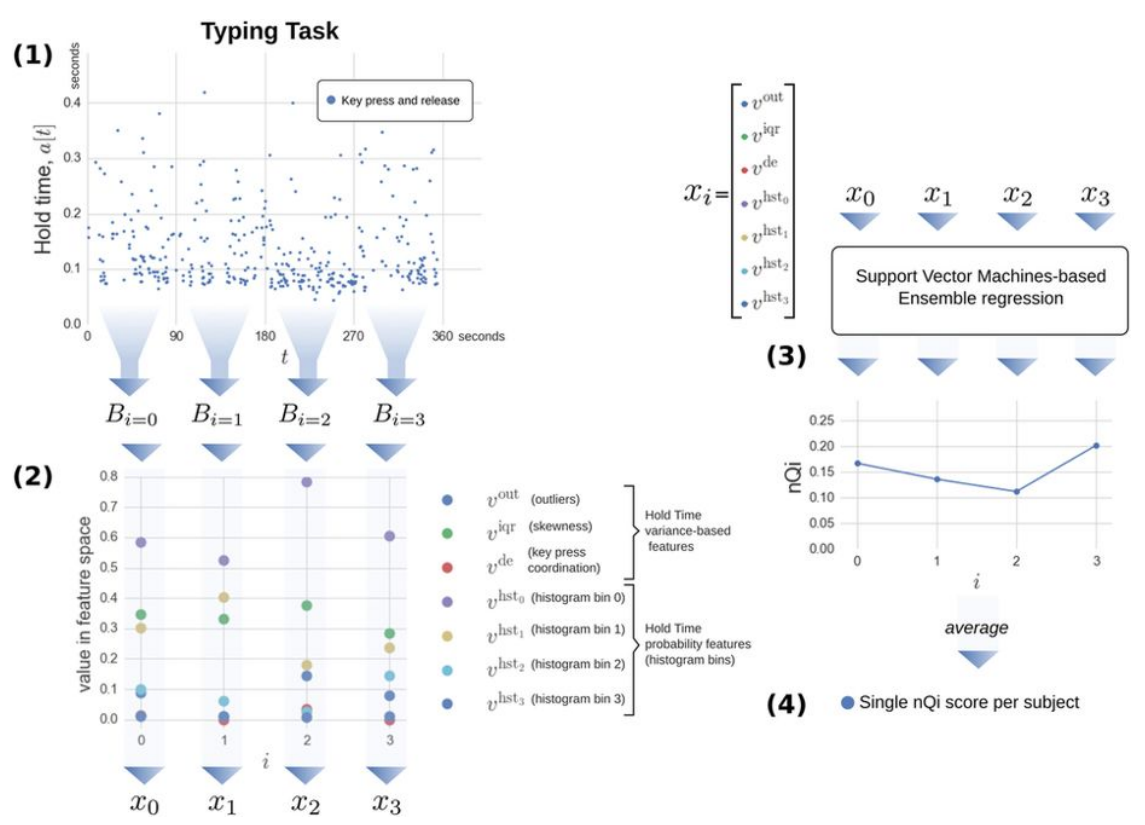


where $N^{w}$ is the size of the window expressed in seconds. In our experiments we used $N^{w}=90$. Then, it is possible to partition the hold time signal a with nonoverlapping square windows as follow: $B_{i}(t)=a[t] \omega\left[t-i N^{w}\right]$ where $t$ is time, $B_{i}$ is a vector containing the ordered list of HT samples and $i$ is a positive integral number which serves as index to the list of vectors. In order to account for the sparsity of the hold times signal, i.e. do not type continuously but in unpredictable bursts, all $B_{i}$ that have less than $N^{w} / 3$ key presses are removed from the set. Let us define a feature vector for each $B_{i}$ :

$$
x_{i}=\left[v^{\text {out }}, \quad v^{\text {iqr }}, \quad v^{d e}, \quad v^{h s t_{0}}, \quad v^{h s t_{1}}, \quad v^{h s t_{2}}, v^{h s t_{3}}\right]^{T}
$$

where $v^{\text {out }}$ is the number of outliers in $B_{i}$ divided by the number of elements in $B_{i}$. An outlier is defined as a HT more than 1.5 interquartile ranges below the first quartile or above the third quartile; $v^{i q r}$ is a measure of the $B_{i}$ distribution skewness described as $\left(q_{2}-q_{1}\right) /\left(q_{3}-q_{1}\right)$, and $q_{n}$ is the $n^{\text {th }}$ quartile; $v^{\text {hst }}$ represents the $n^{\text {th }}$ bin of the $B_{i}$ equally-spaced normalized histogram, i.e. an approximation of the probability density function, with 4 bins from 0 to 0.5 .seconds; $v^{d e}$ is a metric of finger coordination during two consecutive keystrokes. It is measured as $d_{1}-p_{2}$, where $d_{1}$ is the depress event of the first key and $p_{2}$ is the press event of the second key. If $d_{1}-p_{2}<0$, then $v^{d e}=0$. These features were engineered and selected combining graphical exploratory analysis of the HT distributions of PD and CNT groups and clinical domain knowledge.

\section{Ensemble regression}

We designed an ensemble learning approach composed of a set of base models $F^{\prime}:\left\{f_{m} \mid m \geq 0 \wedge m<N^{m}\right\}$ where $N^{m}$ is the total number of models which in our experiments is 200, based on the number of available samples in the dataset and the cross-validation parameters used during the training phase. Each model $f_{m}$ receives as input an independent feature vector $x_{i}$ and performs a feature transformation step and a regression step as follow:

$$
y_{m}^{\prime}=f_{m}\left(x_{i}^{\prime}\right)=b_{m}+w_{m}^{T} x_{i}^{\prime}
$$

is a linear $\varepsilon$-Support Vector Regression model implemented in LibSVM ("LIBSVM: A Library for Support Vector Machines"); The result is a partial estimation of the nQi score. The nQi for each $x_{i}^{\prime}$ is calculated by applying all the regression models in $F$ on the $x_{i}$ vector and then calculating the median score. The models in $F^{\prime}$ are trained using the normalized UPDRS-III scores as targets. Using a Bagging strategy, we generated a different set of $w_{m}$ and $b_{m}$ coefficients for each $f_{m}$ during the training phase. Bagging allows the creation of $N^{m}$ views of the training dataset by generating multiple sets (or bootstrap samples) via random sampling with replacement. This approach reduces the variance in the nQi score and further limits chances of overfitting (Bishop, 2006).

\subsubsection{Real-world data collection}

The widespread use of personal electronics has placed typing among the activities of our daily routine. This enables the possibility of leveraging the data from users' natural interaction with their devices to apply our method in an unobtrusive manner. From a data collection standpoint, it is straightforward to extend our technology to collect timing information in a naturalistic ecologically valid scenario (e.g. home). 
However, from the standpoint of data analysis, passive monitoring poses interesting challenges that could affect the application of our method to evaluate at-home natural typing. From a data sampling perspective, typing happens in unpredictable bursts which introduce a high degree of sparsity in the resulting typing signals. The various contexts in which the typing data are generated at-home may also add difficulty in contrast with the controlled copy task performed in the clinic. Finally, hardware heterogeneity introduces a potential confounder in the at-home setting that we were able to control in our in-clinic setting by using a single machine approach.

We implemented a data collection platform that allowed us to passively collect the typing information from subjects' daily interaction with their laptop. The neuroQWERTY platform provides functionality for user registration/login, distribution of the data collection software and storage and management of the typing data. Once installed, the data collection software runs in the background capturing the timing information of any keyboard input. More specifically, for each keystroke the program stores the timestamps corresponding to the press and release events. To ensure privacy, the collected information did not include the content of each specific key. However, each keystroke was labeled with its corresponding key category; special key, right side key or left side key, to allow filtering of key-types that engage non-standard digit kinematics (e.g. SHIFT). The data collection software had an estimated timing error of 3/0.28 (mean/std) milliseconds. This error was estimated by generating a synthetic stream of keystroke events at the operating system level. An error of 3 milliseconds represents roughly $2-4 \%$ of the typical HT and as such, it is unlikely to significantly obfuscate PD patterns.

The typing information, linked to each user account, was automatically sent to a remote server for analysis. Privacy and data security were assured at three levels: at the client level, the data transmission level, and the data storage level. Any typing data stored on the local machine (which again, did not include the content of the keys) were encrypted and deleted from the device after sending to the remote server. Data transmission was protected through secure hypertext transfer protocol (https). At the server level, data were stored in the database in encrypted format and were only accessible by authorized database administrators or by the user himself after authentication.

Finally, the platform included an administrator module to provide the study coordinators with an interface to access and control participants' typing activity. The administrator dashboard implemented a color code to alert study coordinators about users' prolonged inactivity. Online visualization of the subjects' typing data was also enabled, including the daily key count and the temporal representation of the raw key typing dynamics. A schema of the complete neuroQWERTY platform framework is shown in Figure 4.2.

A detailed representation of the at-home baseline data collected for each subject is shown in Figure 4.3. The plot illustrates the heterogeneity of subjects' typing behaviors, which we previously identified as one of the potential risks for the validation of our approach in a natural at-home setting. Participants typed an average of 24.07 ( \pm 15.13 ) minutes/day, with 2.79 minutes/day for the less active subject and 83.14 minutes/day for the most active subject. This variability was also observed within subjects' typing routines, as several participants did not present a consistent typing activity over the monitored time period. These characteristics in the at-home spontaneous typing data contrast with the quasi-continuous signal captured during the in-clinic typing test. In the Analysis sub-section we will explain how we addressed these differences to allow us to compare the performance of the algorithm for the in-clinic and at-home scenarios. 
FIGURE 4.2: The neuroQWERTY platform. The neuroQWERTY platform was designed to allow for automatic data retrieval of typing data collected at home and remote management by a study coordinator. Operationally, an account in the neuroQWERTY platform was created for each participant in the study. The process included filling in an online form with basic demographics (login information, age and gender), general health information (diagnosed diseases and medication) and some Parkinson's specific data (year of diagnosis, stage of the disease and UPDRS-III). To complete the setup, the data collection software was downloaded and installed in their users' personal laptop to enable remote data collection. Running in the background, the remote monitoring software captured the timing information of any keystroke press/release events, regardless of the application context and content of the typed text. This information, linked to each user account, was encrypted and automatically sent to a remote server through their home internet connection. The neuroQWERTY platform also implemented an administrator module to provide the study coordinators with an interface to control and visualize participants' typing activity.




FIGURE 4.3: At-Home typing activity. Panel A represents the amount of typing data collected from each of the 52 subjects (25 PD, 27 CNT) included in the analysis. The figure highlights the variability in participants' typing routines, as expected from an uncontrolled at-home setting, not only between subjects but also within subjects over time. The red (PD) and blue (CNT) color-scales indicate daily typing activity measured as the number of valid typing windows provided by each subject during the analysis period. We defined a valid window as a sequence of at least 30 keystrokes within 90 seconds of time. To better illustrate the diversity of typing behaviors, panel B illustrates the variability in the amount of typing data with an example from a single PD subject, contrasting the raw typing data corresponding to a low activity day (2 valid windows) versus the data collected on a high activity day (60 valid windows).

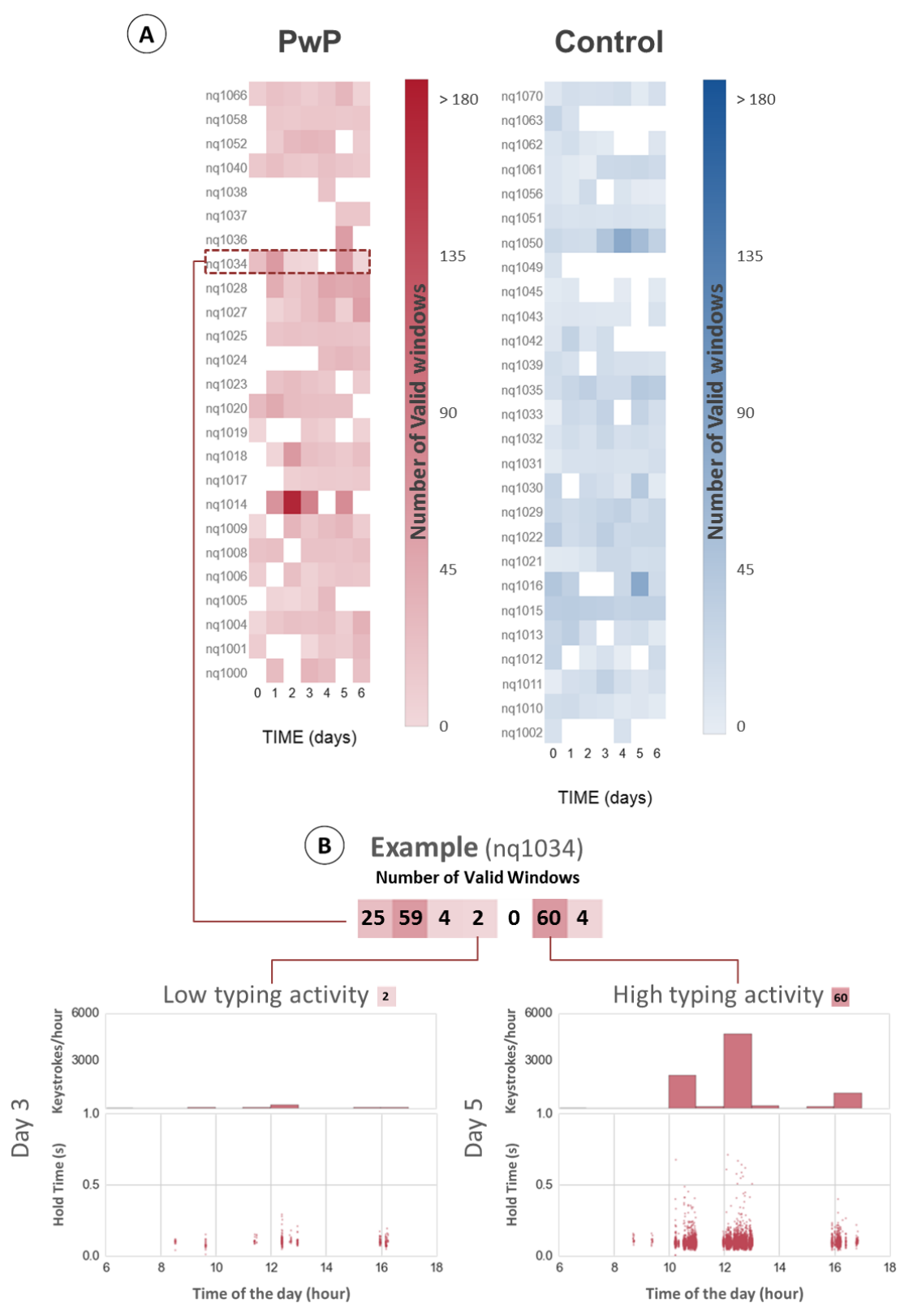


FIGURE 4.4: nQi at-home algorithm pipeline. The figure represents the pipeline to generate a single neuroQWERTY index (nQi) from a stream of typing data. (1) The typing signal is defined as the time series of hold times (HT) corresponding to each keystroke within a typing routine. This signal is split by non-overlapping 90 seconds windows that the algorithm will evaluate as independent typing units. (2) Only windows with at least 30 keystrokes within the 90-second interval are analyzed. (3) The neuroQWERTY algorithm, previously trained on a separate in-clinic dataset, computes a single numerical score from each independent window. (4) The final nQi is computed as the average of the window-level scores.
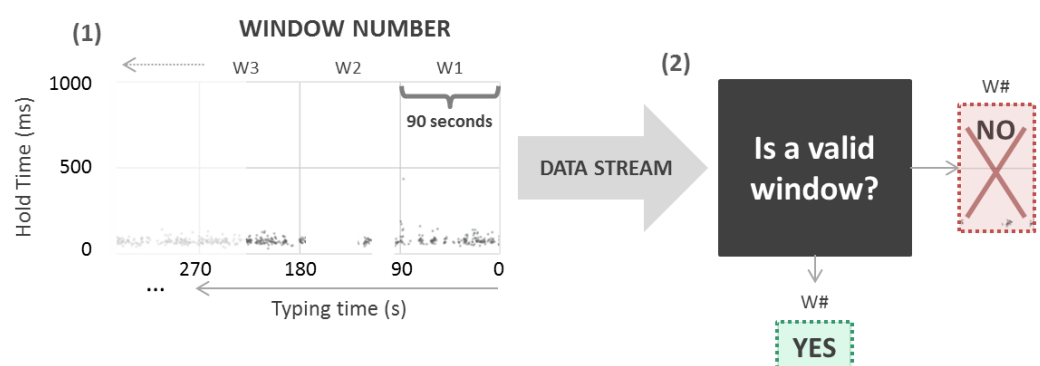

YES

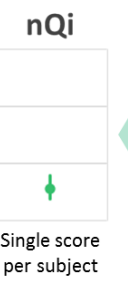

(4)



WINDOW NUMBER

W3

W2 W1

(3) nQi Algorithm

W\#

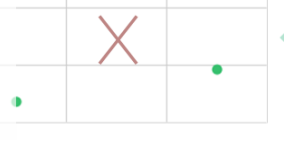

nQi SCORES

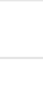

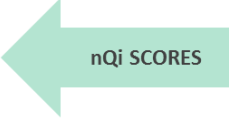

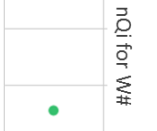

\subsubsection{Analysis}

We evaluate the classification ability of the nQi to separate a group of healthy controls from an early Parkinson's disease population using the typing data collected during subjects' natural interaction with their laptop. The nQi is the output of a computational algorithm that uses the information contained in the sequences of hold times, the time between pressing and releasing each key on a mechanical keyboard, to detect evidence of PD motor impairment. This algorithm was first introduced in Giancardo et al. (Giancardo et al., 2016), where we showed its ability to accurately discriminate early PD from CNT typing patterns from the data collected in a controlled typing task performed in the clinic.

A representation of the algorithm pipeline adapted to the at-home setting is shown in Figure 4.4. The hold time signal is split into 90-second windows that are analyzed as independent typing units. Applying variance analysis, the information within each unit is reduced to a 7-dimensional feature vector that is used as the input of an ensemble model consisting of a family of linear support vector regressors (SVR). An independent window-level score is calculated as the median of the outputs of each linear-SVR. Lastly, the final nQi score is computed as the average of the window-level scores. 
FIGURE 4.5: nQi in-clinic example. Example of the application of the neuroQWERTY algorithm in an in-clinic typing test.

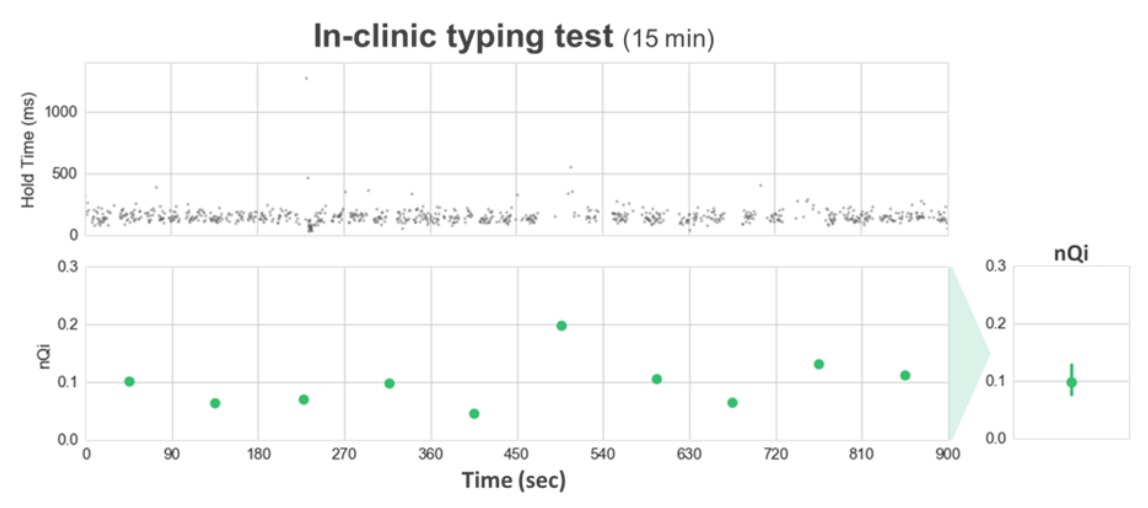

In Figure 4.5, we present an example of the application of the algorithm in a controlled in-clinic task opposed to the same process in an at-home typing setting, Figure 4.6. The comparison of the hold time data representation between in-clinic and at-home illustrates the sparsity introduced in the typing signals by the uncontrolled at-home environment, where the keyboard is only used intermittently as opposed to the continuous stream of data collected in the controlled in-clinic setting. To account for the sparsity of the hold time series, only valid windows, sub-sequences of at least 30 keystrokes within each 90-second interval, are included in the analysis. Special key-types (e.g. SHIFT) that may engage non-standard digit kinematics are excluded from the hold-time data collection. Due to the duration of the at-home baseline period, the volume of data collected at home is generally greater than the in-clinic data available for each subject. We measured an average of 9.62 (2.13) valid windows per subject during the in-clinic typing test and collected an average of 112.33 (70.65) valid windows per subject from the 7-day at-home typing activity. The extended at-home monitoring period increases subjects' at-home sample size, which tends to reduce the individual's internal variance intensified in this uncontrolled environment.

The data analysis comprised two phases. First, we evaluated the influence of the controlled typing task in subjects' normal typing behaviors. Specifically, we compared the measured values of the raw typing metrics, flight time (delay between consecutive key presses) and hold time (time between pressing and releasing a key), and the computed nQi scores between the in-clinic and at-home typing settings. To assess the similarity in the relationship between the in-clinic and at-home metrics we computed the line of best fit and correlation coefficient. We completed this first part of the analysis with a Bland-Altman plot (Bland and Altman, 1986) to evaluate the nQi score's agreement between the two typing settings.

In the second part of the analysis we assessed the classification performance of the neuroQWERTY method using the at-home typing data and compared these results to the ones obtained in the clinic. The results obtained in each typing settings were evaluated using the following metrics: receiver operating characteristic (ROC) analysis and the Mann-Whitney $U$ test to reject the null hypothesis that the controls and the Parkinson's samples come from the same distribution. For the ROC analysis we used a sampling with replacement method to define a distribution of curves from which we computed the average area under the curve (AUC) and its confidence intervals. Each curve is built on an iterative process that monotonically increases the value of the index to define a dynamic threshold. On each iteration, a 
FIGURE 4.6: nQi iat-home example. The neuroQWERTY algorithm can be used indistinctly to evaluate controlled or natural typing data. This figure represents the at-home typing data and corresponding scores for the same subject shown in Figure 4.5 (note different time scales used in 4.5 and 4.6). Although the uncontrolled activity appears in unpredictable bursts that introduce a high degree of sparsity, our window-based approach allows to analyze the at-home data using a similar method as the one applied for the quasi-continuous in-clinic data.

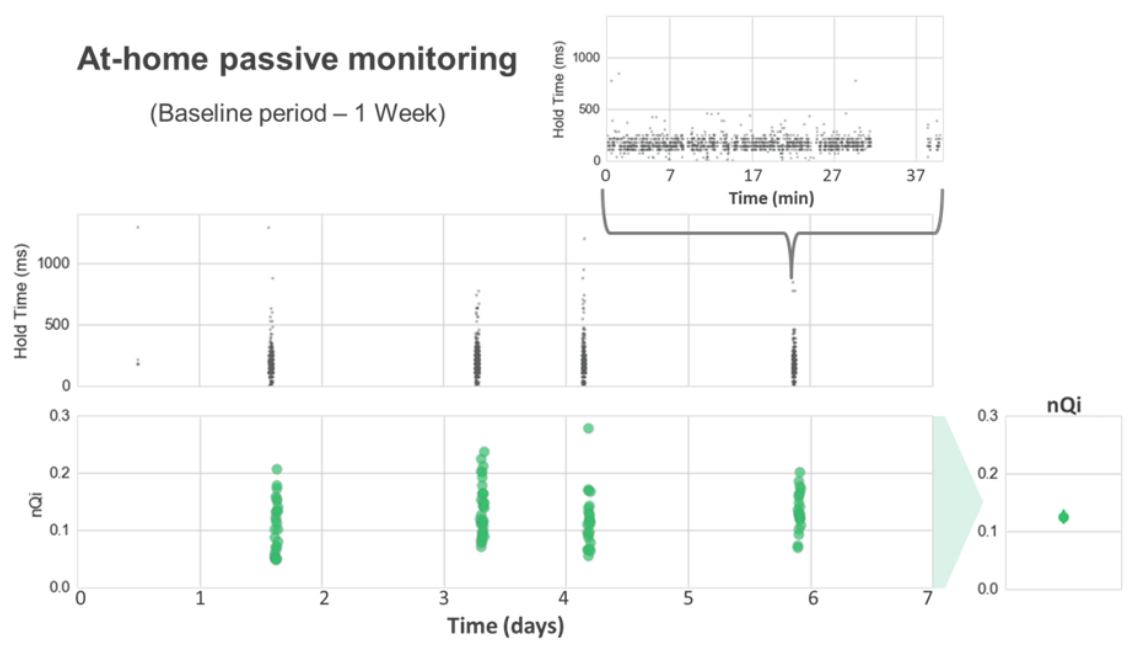

sensitivity/specificity pair is computed using the current threshold value. This pairs are used to draw the resulting ROC curve. The value of the AUC can be interpreted as the probability of the classifier to rank a randomly chosen positive instance higher than a randomly chosen negative one (Fawcett, 2006). To evaluate the equivalence of our method between the in-clinic and at-home settings, we estimated the percentage agreement and the statistical difference of the resulting ROC curves (DeLong test for areas under correlated ROC curves (DeLong, DeLong, and Clarke-Pearson, 1988)).

\subsection{Results}

The results of the raw typing variables agreement between in-clinic and at-home are shown in Figure 4.7. We evaluate the statistical relationship, line of best fit and correlation for the median flight and hold time measured in-clinic and at-home. The values of the two typing metrics are very similar independently of the typing scenario, as shown by correlation coefficient values, 0.913 for the median flight time and 0.897 for the median hold time, and also by the slope of the computed line of best fit, close to 1 in both cases.

A similar analysis applied to the nQi scores is shown in Figure 4.8. The linearity between the in-clinic and at-home settings for this variable is weaker than the observed on the raw typing variables. The correlation coefficient is 0.749 in this case, and the slope of the line of best fit is not as close to the unit, 0.597. However, the agreement analysis suggests a correspondence between the scores measured inclinic and at-home, with a $92 \%$ (48 out of 52 ) of the cases falling between the BlandAltman limits of agreement (LoA).

In terms of classification performance, the nQi worked well with the at-home typing data Figure 4.9 and Table 4.2. The absolute nQi scores tended to be larger 
FIGURE 4.7: Comparison of raw typing metrics between In-Clinic and At-Home typing settings. We compared the raw typing metrics measured in-clinic and at-home to evaluate how different typing tasks influence the way people interact with the keyboard. The figure shows the correlation of the raw typing metrics, hold time (time between pressing and releasing a key) and flight time (delay between two consecutive key presses), between in-clinic and at-home. Each point represents the metric coordinates (in-clinic, at-home) for each of the 52 participants included in the analysis. Both hold and flight time's values are very similar independently of the typing scenario, as shown by the correlation coefficient values. These results suggest that the in-clinic task does not alter the way subjects type in comparison with their natural typing at-home, which supports our hypothesis that the neuroQWERTY algorithm, built on an in-clinic setting, could be applied to evaluate motor impairment using the typing data from an uncontrolled at-home setting.
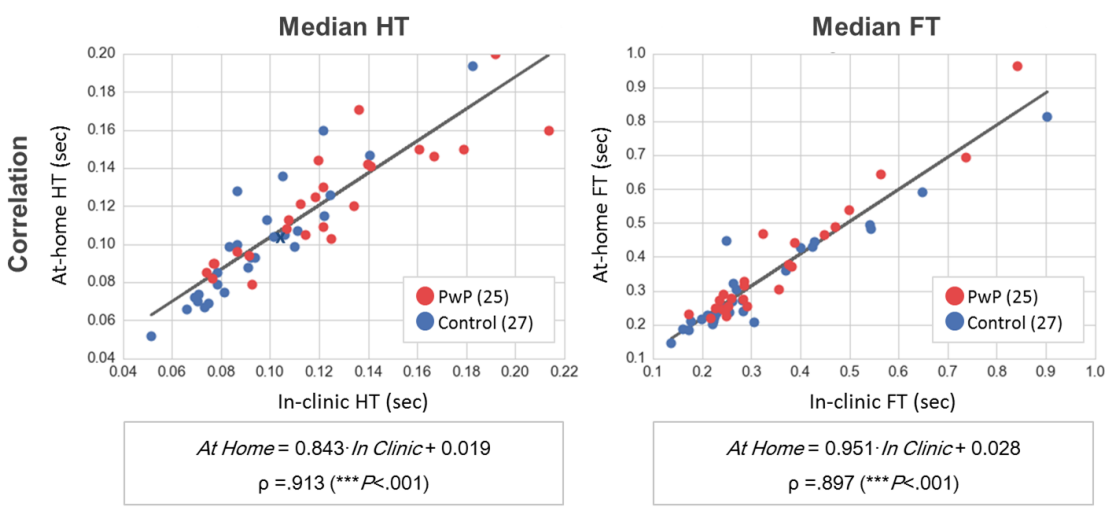

FIGURE 4.8: Comparison of nQ index (nQi) between In-Clinic and At-Home typing settings. We evaluated the influence of the typing setting in the nQi scores by applying a similar analysis as described in Figure 5 for the raw typing metrics. Panel A shows the correlation of the nQi scores computed in-clinic and at-home. Panel B includes the results of the Bland-Altman analysis to evaluate the agreement of our method in the two typing scenarios. The black line shows the mean difference $(d)$ and the top and bottom dashed lines show the limits of agreement $\left(\mathrm{LoA}, d \pm 1.96 * S D_{d}\right)$.

(A)

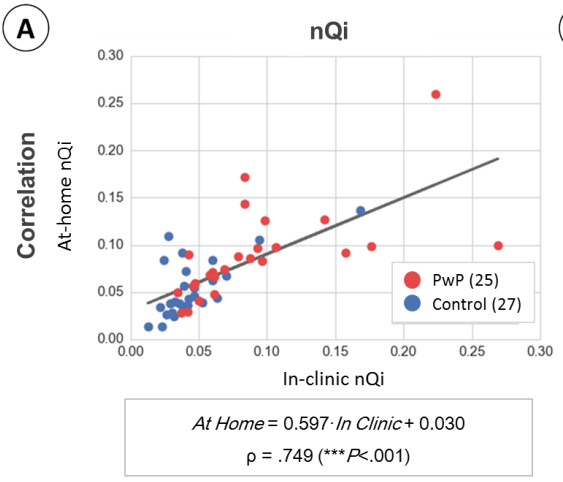

B

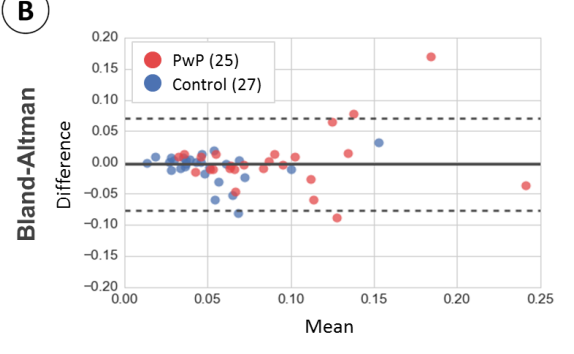


FIGURE 4.9: Comparison of nQi performance between In-Clinic and At-Home typing settings. In this figure we compare the classification performance achieved in the two tested scenarios to validate our hypothesis that the neuroQWERTY algorithm, trained on a separate in-clinic dataset, can also be applied to detect $\mathrm{PD}$ related motor impairment in an at-home typing setting. Panel A scatterplot illustrates the in-clinic and at-home nQi scores in a patient level. The two black lines represent the classification thresholds computed in-clinic $(\mathrm{nQi}=0.0473)$ and at-home (nQi $=0.0667)$. These thresholds were estimated for closest-to- $(0,1)$ cut-off points that maximize sensitivity/specificity pairs. Panel B presents the comparison of the receiver operating characteristic (ROC) curves showing the classification rate for the in-clinic and at-home nQi. The plotted curves are the average result of the bootstrapped ROC analysis and the shadowed areas represent the corresponding confidence intervals [5th-95th]. Statistical significance of the Mann-Whitney $U$ test is estimated to reject the null hypothesis that the two groups, PD and CNT, come from the same population. It's noted as: $\mathrm{p}<0.001\left(^{* *}\right), \mathrm{p}<0.01\left(^{* *}\right)$ and $\mathrm{p}<0.05\left(^{*}\right)$. The results show how our in-clinic-built algorithm is able to discriminate PD and CNT from the passively monitored at-home data nearly as well as it was able to discriminate from the controlled in-clinic data.
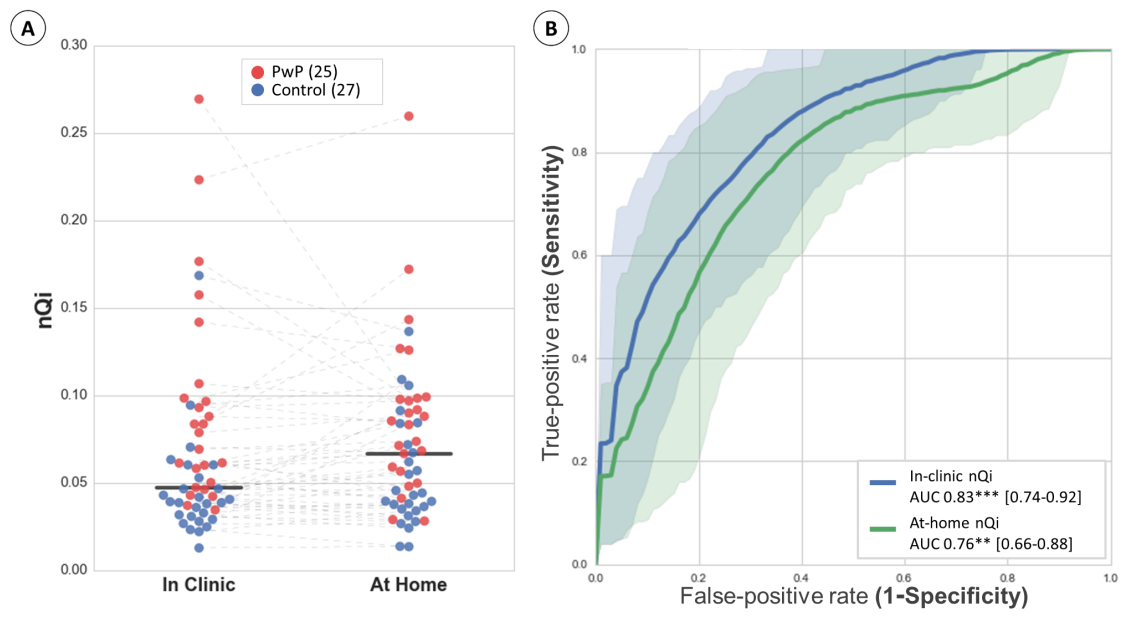

for at home data relative to the corresponding in-clinic values Figure 4.8, and Figure $4.9 \mathrm{~A}$, but in both cases the scores for PD were generally greater than for controls. The similarity in classification performance for in-clinic versus at-home data can also be seen by comparing the ROC curves Figure 4.9B, Table 4.2. The neuroQWERTY algorithm discriminates our early PD population from controls with an AUC of 0.76 [0.66-0.88] using the typing data from the at-home natural interaction a mechanical keyboard. In the clinic, the results of the analysis a controlled typing task in the same cohort achieved an AUC of 0.83 [0.74-0.92]. According to the DeLong test for areas under correlated ROC curves, the ROC AUC difference between in-clinic and at home was not significant $(\mathrm{p}=0.176)$. The percentage agreement of the results of our method between the two typing settings was $79 \%$. 
TABLE 4.2: nQi Performance Comparison. The table compares nQi performance between the in-clinic and at-home typing settings. The classification performance achieved At-Home (0.76 [0.66-0.88] AUC and $0.73 / 0.69$ sensitivity/specificity) is comparable to the results obtained in a controlled In-Clinic setting (0.83 [0.74-0.92] AUC and $0.77 / 0.72$ sensitivity/specificity). The cutoff point was estimated using the closest-to- $(0,1)$, i.e. the use case that maximizes the sensitivity/specificity pair (Perkins and Schisterman, 2006). The statistical significance is computed with two-sided Mann-Whitney U test to reject the null hypothesis that PD and CNT subjects come from the same population. The differences between the in-clinic and at-home ROC curves were not significant according to the DeLong test for areas under correlated ROC curves. The method reached a percentage agreement close to $80 \%$ between the two typing scenarios.

\begin{tabular}{lcc}
\hline Metric & nQi In-Clinic & nQi At-Home \\
\hline \hline Mean (SD) for PD $(\mathrm{n}=25)$ & $0.092(0.058)$ & $0.090(0.048)$ \\
\hline Mean (SD) for CNT $(\mathrm{n}=27)$ & $0.046(0.029)$ & $0.054(0.030)$ \\
\hline AUC $\left(5_{t h}-95_{t h}\right)$ & $0.83[0.74-0.92]$ & $0.76[0.66-0.88]$ \\
\hline Significance & $\mathrm{p}<0.001$ & $\mathrm{p}<0.01$ \\
\hline Sensitivity/specificity & $0.77 / 0.72$ & $0.73 / 0.69$ \\
\hline DeLong test & $\mathrm{p}=.18$ & $\mathrm{p}=.18$ \\
\hline Percentage agreement & $79 \%$ & $79 \%$ \\
\hline \hline
\end{tabular}

\subsection{Discussion}

The results of this study represent a step towards a transparent and ubiquitous motor sign assessment tool for PD. In our in-clinic work (Giancardo et al., 2016), we introduced the neuroQWERTY method, a machine learning algorithm trained to quantify PD severity through the analysis of the typing patterns found in the time series of hold times (HT). Our method was able to discriminate an early PD population from a matched control group using the typing data collected during a controlled in-clinic task. In this work, we tested the validity of our algorithm in an uncontrolled at-home setting. The neuroQWERTY platform allowed us to unobtrusively collect the typing information from a cohort comprised of $30 \mathrm{PD}$ and 30 matched controls. Most (52 out of 60, 90\%) of the study subjects (25 PD, 27 CNT) provided enough data during the follow-up period to evaluate the neuroQWERTY index (nQi) at home. Our neuroQWERTY algorithm, built using a separate in-clinic dataset, was able to distinguish PD from CNT through the analysis of natural at-home typing patterns with an AUC of 0.76 and 0.73/0.69 sensitivity/specificity. Despite the sparsity and heterogeneity introduced by each subject's routine use of the computer, the neuroQWERTY method performed nearly as well in the at-home setting as it did when applied in a controlled in-clinic typing task (AUC 0.83 and 0.77/0.72 sensitivity/specificity). The nQi scores presented no significant differences between the de-novo PD group (19) and the subset of PD participants on medication.

The comparison of the raw typing metrics between the two typing scenarios suggests that the in-clinic typing test does not affect the way people type with regard to their normal use of the computer at-home. The correlation coefficient for the median HT between in-clinic and at-home was $p=0.913(\mathrm{p}<0.001)$. A similar analysis applied to the resulting nQi shows a weaker correlation between the scores computed from the in-clinic and at-home typing data $(p=0.749(p<0.001)$ with a $92 \%(48$ out if 52$)$ of the samples within the Bland-Altman limits of agreement). This could be due to 
the sensitivity of the algorithm to small changes in the hold time values between the two typing settings. Despite the weaker correlation, the classification performance of the neuroQWERTY method applied at-home was similar to the classification performance in-clinic (statistically indistinguishable by the DeLong test for areas under correlated ROC curves $\rightarrow \mathrm{p}=0.176$, percentage agreement $\rightarrow 79 \%$ ).

These results support our initial hypothesis that PD-related motor signs affect the way patients interact with mechanical keyboards and are, therefore, detectable through the analysis of their regular typing patterns. The ability of the neuroQWERTY algorithm to extrapolate the patterns learnt from a separate in-clinic dataset to correctly identify PD-characteristics in the at-home typing data provides external validity to our method. Being able to generalize to data collected from the hardest possible scenario can also be seen as an opportunity to improve these results by implementing an at-home-specific algorithm that, trained on passively collected data, will be able to identify the useful information and learn to filter the several different sources of noise introduced by the uncontrolled at-home setting. Although the current clinical standard, UPDRS, outperforms our technique, the goal of neuroQWERTY is not to replace UPDRS but to provide a method that enables PD assessment when a clinician is not available. Nevertheless, it would be interesting to explore the potential for the nQi approach to provide a meaningful indication of UPDRS. Despite being based just on distal upper limb movement, with our limited data set we did find a significant moderate correlation between the nQi scores and UPDRS-III.

Using the timing information from users' natural typing activity provides our approach with a number of advantages over alternative solutions, but it also poses some limitations. An obvious concern is the level of compliance, since the method depends on sufficient use of the computer. In the study cohort, a high percentage of the participants $(90 \%)$ provided enough data during the 7-day follow-up to shape a representative typing pattern. Our user adherence results highlight the advantages of passive data collection in contrast with other existing active task-based methods. Task-based methods are commonly limited by their dependence on users' active engagement to collect information through a series of standardized tasks, which introduces potential artifacts due to subject-awareness of being monitored (McCarney et al., 2007b) and hinders user compliance. As an example, in the context of the mPower study less than $10 \%$ of the participants provided 5 or more finger-tapping data points over a six months follow-up period (Bot et al., 2016b).

Although our passive data collection approach significantly increases user adherence, some strategies could be employed in order to maximize it. A possible solution to reduce the rate of excluded participants would be collecting data not only from laptop use, but from any electronic device that entails typing. In the next chapter we will show how a similar approach can be used to detect PD via smartphone touchscreen typing. Integrating data from multiple devices would provide a more continuous stream of data; therefore, a deeper insight to assess PD signs.

Proving that our method can distinguish an early PD cohort, with an average years from diagnosis of 1.66 (1.20) and mean UPDRS-III score of 20.48 (6.56), from a matched CNT group is an indicator that at-home typing patterns can capture PDspecific motor characteristics that are mild in this stage of the disease. This could have an impact in early detection of PD as machine learning algorithms can be trained to detect very subtle variations in the input data, in this case changes in the typing patterns, caused by early motor manifestations of PD that may often go unnoticed by clinicians (Ross et al., 2012). The neuroQWERTY software could be installed on PD-risk populations' devices to enable earlier diagnosis, when putative neuroprotective treatments could stop neurodegeneration. Clinical studies in 
an as-yet-undiagnosed population would be needed to validate the sensitivity and applicability of our tool for this specific use case.

Although our classification results show promise, our longer term goal is to develop tool to objectively track progression of PD signs. This would provide clinicians with invaluable information to tailor treatments to patients' specific conditions. Today, there is no known cure for PD, but available medications can help manage its symptoms. Individualized treatment regimens are crucial to provide optimized symptom control (Tarakad and Jankovic, 2017). Medications adjustments rely mainly on the information gathered by movement disorder experts during clinical visits. This limits decision-making to subjective follow-up examinations scheduled every 2-6 months. Ideally, our approach could be applied not only to classify, but also to track PD progression and therapeutic efficacy. This would require further validation in a longitudinal study to evaluate if the precision of the neuroQWERTY approach to monitor PD progression over time.

Relying on the analysis of the temporal patterns from the daily interaction with electronic devices, our approach introduces a new way to objectively and unobtrusively detect motor impairment in PD, providing access to quasi-continuous ambulatory data without harming user compliance. The main purpose of this analysis was to evaluate the validity of the neuroQWERTY index (nQi), an in-clinic built digital marker for early PD motor impairment, in an uncontrolled at-home setting. The classification performance of the algorithm was statistically similar in its ability to discriminate $25 \mathrm{PD}$ and $27 \mathrm{CNT}$ from the at-home typing data (AUC of 0.76 and $0.73 / 0.69$ sensitivity/specificity) nearly as well as it was able to separate them using the in-clinic typing patterns (AUC 0.83 and $0.77 / 0.72$ sensitivity/specificity). These results prove that the data collected from subjects' routine use of the computer is also valid to detect PD-related motor signs, getting us a closer to our ultimate goal of providing an objective ambulatory tool to monitor PD progression. 


\section{Chapter 5}

\section{Touchscreen Typing as an Indicator of Early Parkinson's Disease}

Mobile technology is opening a wide range of opportunities for transforming the standard of care for chronic disorders. Using smartphones as tools for longitudinally tracking symptoms could enable tailored drug regimens and improve patient monitoring. Parkinson's disease (PD) is an ideal candidate for these tools. At present, evaluation of PD signs requires trained experts to quantify motor impairment in the clinic, limiting the frequency and quality of the information available for understanding the status and progression of the disease. Mobile technology can help clinical decision making by completing the information of motor status between hospital visits.

We propose a solution that takes advantage of the ubiquity and pervasiveness of smartphone technology. Importantly, in contrast to many other mobile-based approaches, our solution is transparent to the user, and does not require the user to take any action to initiate a test. Our primary objective is for this transparent monitoring to provide information comparable to current motor tests. More specifically our approach should simplify the monitoring process by passively collecting information from the routine use of smartphone devices. Building on our previous work on mechanical keyboard typing (Giancardo et al., 2016; Arroyo-Gallego et al., 2018a), we introduced a set of numerical features derived from similar keystroke dynamic variables on mobile phone touchscreens. We learnt characteristic PD typing patterns to facilitate detection and quantification of the motor signs related to this disease. PD motor phenotype is described by slowness, lack of spontaneous movement, rigidity, and tremor. This clinical picture should affect the unconstrained finger performance while interacting with smartphone devices.

This chapter proposes a smartphone-based approach to assess PD motor signs. The results presented in this chapter were published in "Detection of motor impairment in Parkinson's Disease via mobile touchscreen typing" (Arroyo-Gallego et al., 2017). Our solution uses touchscreens as hardware support, and relies on the typing signal as input to evaluate motor function anomalies. Our study is a first step towards a transparent and ubiquitous motor sign assessment method that is objective, convenient, and can produce quasi-continuous ambulatory data. The main contribution of this work is a new methodology to detect PD-related motor impairment through the analysis of the typing signal collected via smartphone devices. We tested our solution on a validation cohort that includes data from 21 PD and 23 control subjects. The performance and relevance of the developed tool is verified by comparing the obtained results with respect to the alternating finger-tapping (AFT) motor test. 


\subsection{Methods}

This sections includes a general description of the data acquisition, followed by the presentation of the proposed methodology.

\subsubsection{Data Acquisition}

We collected 51 typing signals from a population composed of 24 people diagnosed with Parkinson's and 27 healthy controls. Subjects gave informed consent prior to experiments, and experimental procedures were approved by the Committee On the Use of Humans as Experimental Subjects (COUHES) at the Massachusetts Institute of Technology, protocol no. 1504007090. During the meeting the subjects independently underwent a clinical evaluation including the UPDRS-III test conducted by a movement disorder expert. After the clinical assessment, each participant created an account on our website (www.neuroqwerty.com). The Alternating finger-tapping (AFT) test was performed on a physical keyboard. Subjects had to alternatively press two keys, separated approximately $25 \mathrm{~cm}$, using their index finger. They repeated the test for both hands. The final score was computed as the average number of buttons pressed between the two hands. The typing data was collected using dedicated smartphone software. Participants transcribed a randomly-selected text excerpt for five minutes and were instructed to type as they would normally do in order to reflect actual routine use of the device. PD subjects were tested during their "ON" state, under best medical treatment.

Seven participants, 4 from the control group and 3 Parkinson's subjects, did not have enough data to compute the feature analysis and were excluded from the dataset. All of them presented a typing rate below 20 keys per minute for at least half of the typing time. Table 5.1 summarizes the demographic information of the remaining 44 subjects that were included in the analysis.

For the study, we developed a custom screen keyboard in order to enable typing data collection. The application was based on the open source software keyboard AnySoftKeyboard (github.com/AnySoftKeyboard). Software keyboards like this are invoked by any application that requires textual input, therefore it allowed us to capture the timestamps corresponding to press and release events for each keystroke. The system tested has a clock speed and a theoretical low-level sampling frequency of $1.2 \mathrm{GHz}$. Our implementation uses a software timer with a time granularity of 1 millisecond. The encrypted information was sent to a remote server for the analysis. All the subjects were tested on an Android terminal, i.e. Motorola Moto G II running Android 5.0. In Figure 5.1 we provide a graphical representation of the study procedure.

\subsubsection{Data Analysis}

The method description is divided into three different phases as follows: An initial signal conditioning phase in order to minimize signal noise and artifacts. Then, statistical analysis is used to describe the processed signal using a limited number of typing features. In the last stage, predictive machine learning models are trained and tested on the typing feature selected to determine their suitability for detecting PD status. 
TABLE 5.1: Dataset Demographics. The complete study cohort comprised 51 subjects. From the total participants, 44 provided enough typing information to perform the analysis. A minimum of 5 key presses every 15 seconds during at least half of the duration of the typing task was required to apply the proposed method. Seven subjects, 3 from the Parkinson's group and 4 healthy controls, did not provide enough data and were excluded from the analysis (see Materials and Methods). The table provides a summary of the demographic information of the participants included in the analysis, 21 people diagnosed with Parkinson's (PD) and 23 control subjects (CNT). PD subjects and controls are statistically similar in age, according to the two-sided Mann-Whitney U test. The same test suggests gender might be a confound variable in this study. These differences were accounted in the analysis. The table also shows the results of the clinical evaluation that includes UPDRS-III, alternating finger-tapping and the Modified Hoehn and Yahr scale. The Hoehn and Yahr scale is a widely used clinical rating scale that defines broad categories of disability in PD in a 0 to 5 range.

\begin{tabular}{lccc}
\hline & $\begin{array}{c}\text { Avg. (std) } \\
\text { Parkinson's }\end{array}$ & $\begin{array}{c}\text { Avg. (std) } \\
\text { Controls }\end{array}$ & Significance \\
\hline \hline Age & $59.24(11.43)$ & $54.35(13.95)$ & $\mathrm{p}=0.32$ \\
\hline Women \# & 11 & $19(83 \%)$ & $\mathrm{p}<0.05$ \\
\hline Men \# & 10 & $4(17 \%)$ & $\mathrm{p}<0.05$ \\
\hline UPDRS-III & $17.76(7.92)$ & $1.22(1.70)$ & $\mathrm{p}<0.001$ \\
\hline Alternating finger-tapping & $49.17(10.65)$ & $67.54(14.11)$ & $\mathrm{p}<0.001$ \\
\hline Hoehn and Yahr & $2.05(0.31)$ & N.A. & N.A. \\
\hline \hline $\mathrm{n}$ (total $\mathrm{n}=44)$ & 21 & 23 & \\
\hline \hline
\end{tabular}


FIGURE 5.1: Schema of study design. The figure presents a schema of the study procedure that comprises a clinical evaluation, fingertapping test and our typing test. For the clinical evaluation a movement disorder specialist filled-in the motor section in the Unified Parkinson's Rating Scale (UPDRS-III). The alternating finger-tapping test was included as an external reference to quantify upper limbs dexterity. It was performed on a physical keyboard. The typing test consisted of a five minutes task where participants were asked to transcribe a non-standardized text excerpt using a touchscreen device. The custom screen keyboard and smartphone model used in the test are shown in this figure.

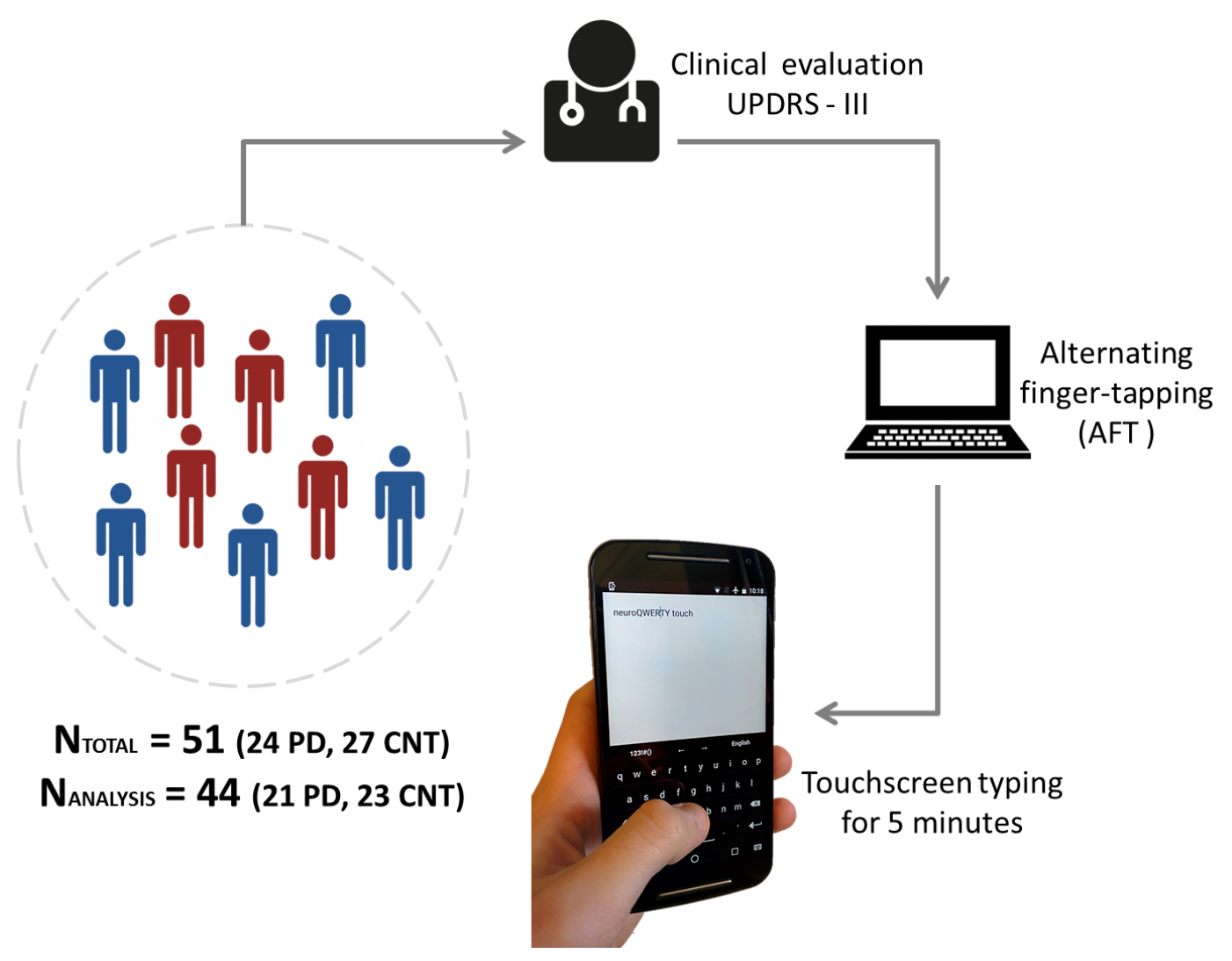




\section{Signal Conditioning}

In this study, we define the typing signal $(X[t])$ as the sequence of flight time (FT) values corresponding to each key tap. In the context of our work, we define FT as the release latency between key taps, i.e. for two consecutive keystrokes the time measured between first and second key release times. The captured typing data requires further processing in order to remove noise, minimize the effect of confounding factors on the analysis and define a standardized representation of the whole signal. Noise can be introduced by many sources, such as software inaccuracies or unnatural typing episodes (e.g. special keys). Additionally, to reduce noise levels, the signal is processed by a series of conditional filters that remove potentially noisy samples if the FT value exceeds a 3-second threshold, or if they correspond to special key-types that engage non-standard digit kinematics (e.g. SHIFT). The 3-second FT threshold was experimentally set based on the definition of valid window introduced in Chapter 4; 30 keystrokes within 90 seconds is translated to $F T \simeq 90$ seconds $/ 30$ keystrokes $=3$ seconds. To minimize the effect of typing skills in the results of the analysis, each signal is normalized by subtracting its mean value to every data sample in $X[t]$ for each subject. Figure 5.2 compares the probability density functions for the normalized FT (NFT) data grouped by condition.

$$
X^{\prime}[t]=X[t]-\bar{X}: X^{\prime} \in\left[\theta_{A}, \theta_{B}\right]
$$

where $X^{\prime}$ is the normalized signal and $\bar{X}$ its average. The value $t$ represents the time at which a key has been pressed to generate the relative FT signal. The parameters $\theta_{A}$ and $\theta_{B}$ give the estimated range of values in which the $99 \%$ of the normalized FT data is concentrated. These two parameters have been estimated in an external typing database of 27 healthy subjects.

We define a new type of signal representation $\left(X_{S}^{\prime}\right)$ based on the FT time series as to adapt the normalized data to the following analysis stages. Given $X^{\prime}[t]$, the signal structure, $X_{S}^{\prime}$, is defined as a set of vectors $X_{S i}^{\prime}$ with a varying number of elements but a fixed length in the time domain:

$$
X_{S i}^{\prime}[t, N]=X^{\prime}[t] w[t-i N]
$$

where $i$ is a strictly positive integer which serves as an index to the list of vectors, $N=15,000$ is the length of the window time expressed in milliseconds and $w[n]$ is defined as:

$$
w[n]=\left\{\begin{array}{cc}
1, & 0 \leq n<N \\
0, & \text { otherwise }
\end{array}\right.
$$

\section{Feature Extraction}

Evaluating $X_{S}^{\prime}$ using distribution and covariance based approaches we define two different feature families.

Skewness and Kurtosis These measurements correspond to the third and forth moments of a distribution. Skewness can be interpreted as an indicator of distribution symmetry, while kurtosis measures the variable distribution flatness. Each 
FIGURE 5.2: Flight time signals distribution. The figure shows the probability density distribution (PDF) estimated for the normalized flight time (NFT) signals both for each subject (light color) and grouped by condition (dark color). Normalization minimizes the influence of confounding variables related to typing skills. Parkinson's subjects' (PD) distributions present a greater sparsity than controls' (CNT) distributions. A Mann-Whitney $U$ test suggests a significant difference between the NFT values measured on PD participants and controls $(\mathrm{p}<0.001)$.

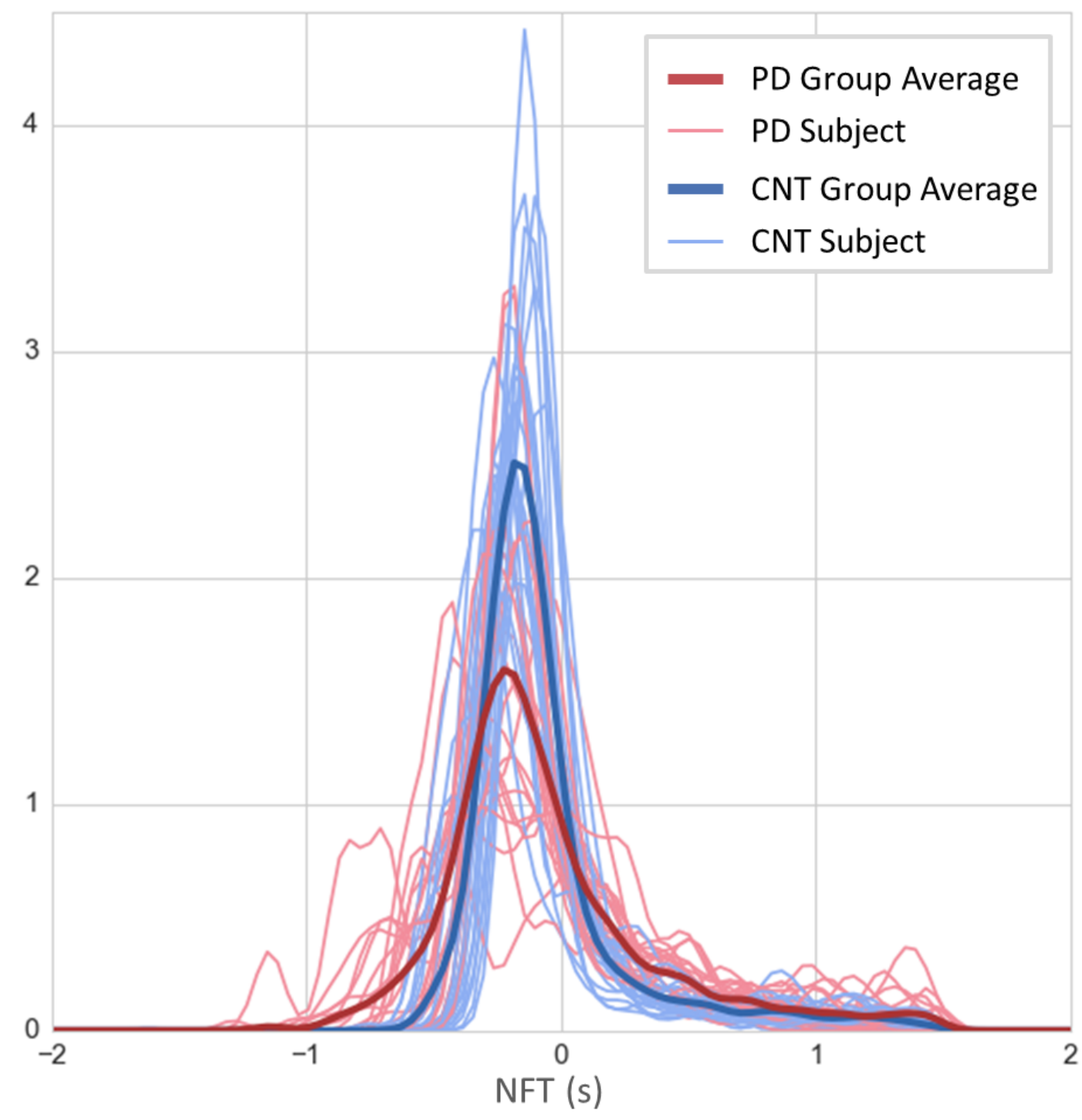


element in the typing structure $\left(X_{S i}^{\prime}\right)$ is evaluated as an independent realization of the same random variable, with its corresponding distribution that we will call "subdistribution" in the context of $X[t]$. Then, for each sub-distribution a pair including skewness $\left(S k_{i}\right)$ and kurtosis $\left(K t_{i}\right)$ descriptors are computed.

For a sample of $n$ values, a natural method of moments estimator of the population skewness is:

$$
S k_{i}=\frac{\sum_{m=1}^{M_{i}}\left(X_{S i}^{\prime}[m]-\bar{X}^{\prime}{ }_{S i}\right)^{3}}{\sigma_{S i}^{3}}
$$

For a sample of $\mathrm{n}$ values the sample excess kurtosis is:

$$
K t_{i}=\frac{\sum_{m=1}^{M_{i}}\left(X_{S i}^{\prime}[m]-\bar{X}_{S i}^{\prime}\right)^{4}}{\sigma_{S i}^{4}}-3
$$
$X_{S i}^{\prime}$

where $M_{i}$ is the length of the $i^{\text {th }}$ vector in $X_{S}^{\prime}$ and $\sigma_{S i}$ is the standard deviation of

With $I$ being the number of sub-distributions that compose the structured typing signal, the analysis described above generates a total of $I$ measures for each metric. These values are reduced to four final features computed as the average and standard deviation of the $I$ skewness measurements $\left(\overline{S k}, \sigma_{S k}\right)$ and the $I$ kurtosis measurements $\left(\bar{K} t, \sigma_{K t}\right)$.

Covariance The typing signal structure $\left(X_{S}^{\prime}\right)$ is transformed into a matrix $(H)$ by applying Kernel Density Estimation (KDE). A similar approach was presented in Giancardo et al., 2015 to define the Key Hold Time Evolution Matrix.

We apply KDE to estimate the probability density function (PDF) that represents the underlying distribution of each element in the typing structure. Given a typing sub-sample $X_{S i}^{\prime}$ of size $M_{i}$, its $\operatorname{PDF} f_{i}$ is computed as follows:

$$
f_{i}(y, b)=\sum_{m=1}^{M_{i}} K\left(\left(y-X_{S i}^{\prime}[m]\right) / b\right)
$$

where $b$ is a bandwidth parameter that controls $K$, a Gaussian kernel:

$$
K(x, b) \propto \exp \left(-\frac{x^{2}}{2 b^{2}}\right)
$$

Each function $f_{i}$ is quantizied using pre-defined mapping levels $\vec{v}$. This allows a standardized $I \times L$, matrix representation of the typing signal as:

$$
H_{i, j}=f_{i}(\vec{v}[j])
$$

We used our external dataset, not used for training or testing, comprised by 27 healthy subjects, to adjust the value of the bandwidth parameter $b$ Sheather and Jones, 1991, as well as the number of quantization levels $(L=10)$ and the limits of the mapping vector $(\vec{v})$.

The corresponding covariance matrix $\left(\mathrm{COV}_{H}\right)$ is estimated for the resulting NFT distribution matrix $(H)$ as follows: 


$$
\operatorname{COV}_{H_{i, j}}=\operatorname{cov}\left(H_{i *}, H_{j *}\right)=\frac{1}{L-1} \sum_{l=1}^{L}\left(H_{i, l}-\bar{H}_{i *}\right)\left(H_{j, l}-\bar{H}_{j *}\right)
$$

where $H_{i *}$ is a vector that contains the $H$ matrix values for the $i^{\text {th }}$ row:

$$
H_{i *}=\left[H_{i, 1}, H_{i, 2}, \cdots, H_{i, l}\right]^{T}
$$

Being $C v$ a covariance vector including the upper triangle elements of $C O V_{H}$, i.e. the coefficients in the upper portion above the main diagonal of the matrix:

$$
\begin{gathered}
\operatorname{COV}_{H}=\left[\begin{array}{cccc}
0 & \operatorname{COV}_{H_{1,2}} & \cdots & \operatorname{COV}_{H_{1, L}} \\
0 & 0 & \cdots & \operatorname{COV}_{H_{2, L}} \\
\vdots & \vdots & \ddots & \vdots \\
0 & 0 & \cdots & 0
\end{array}\right] \\
C v=\left[\operatorname{COV}_{H_{1,2}} \operatorname{COV}_{H_{1,3}}, \cdots, C O V H_{I-1, L}\right.
\end{gathered}
$$

We defined the following covariance typing features: covariance mean $(\overline{C v})$, covariance standard deviation $\left(\sigma_{\mathrm{Cv}}\right)$ and the sum of the absolute values of the covariance vector elements $\left(\sum|C v|\right)$. A graphic representation of the typing signal characterization is presented in Figure 5.3. 


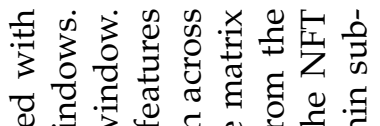

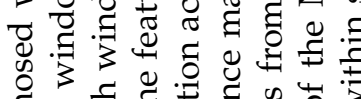
年

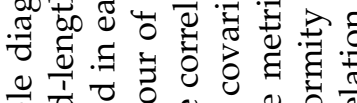

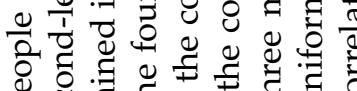
\&

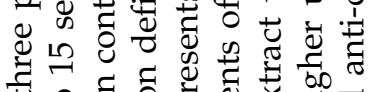

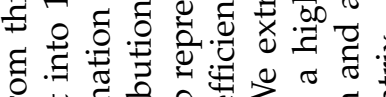

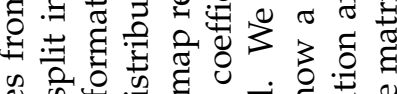

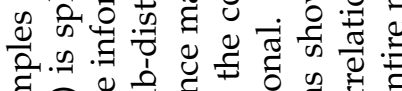

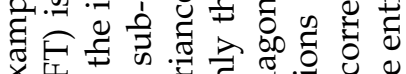
ช

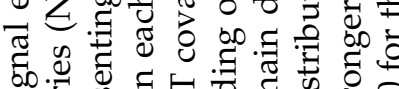
点.

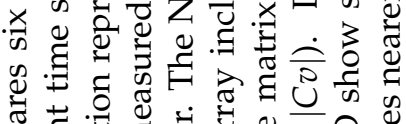

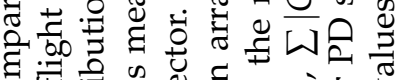

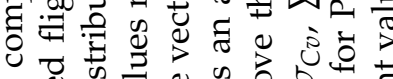

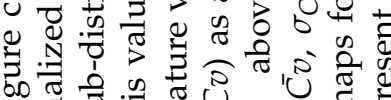

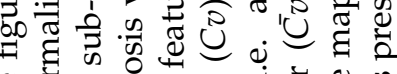

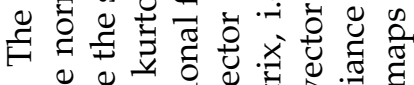
कि 宊

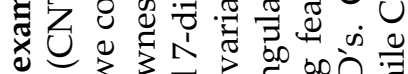
สำ .

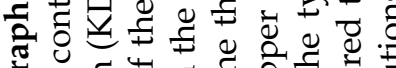



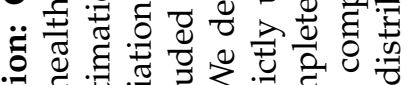



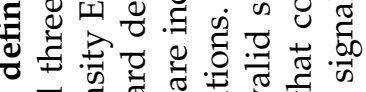


ฮั क क in

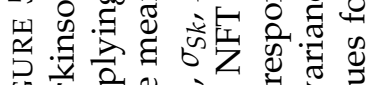



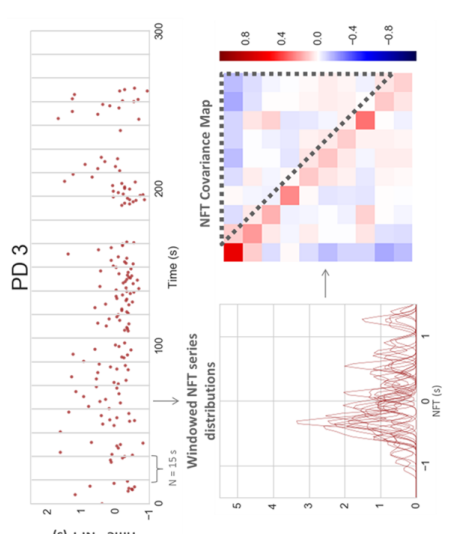



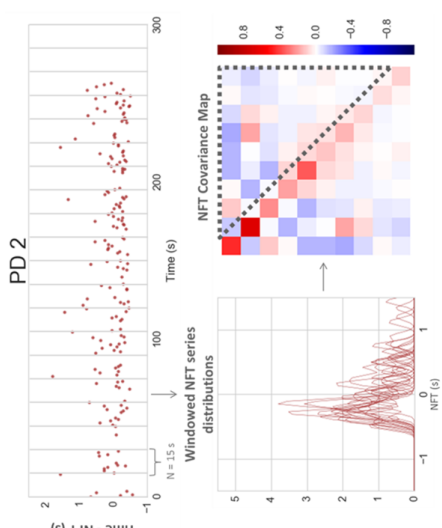

(s) 1 IN - amII

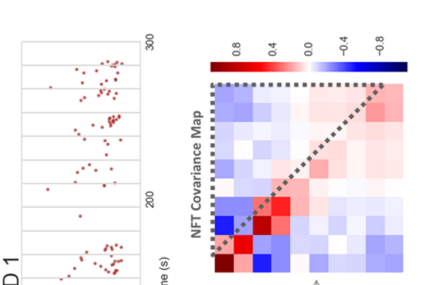

à

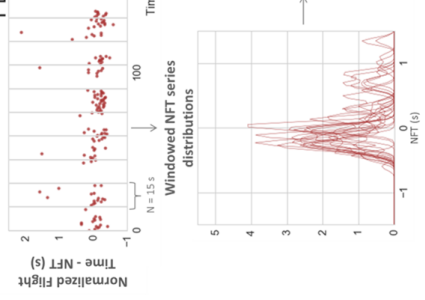

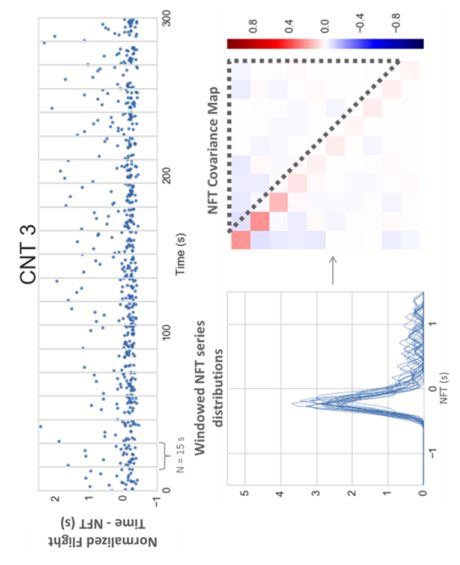
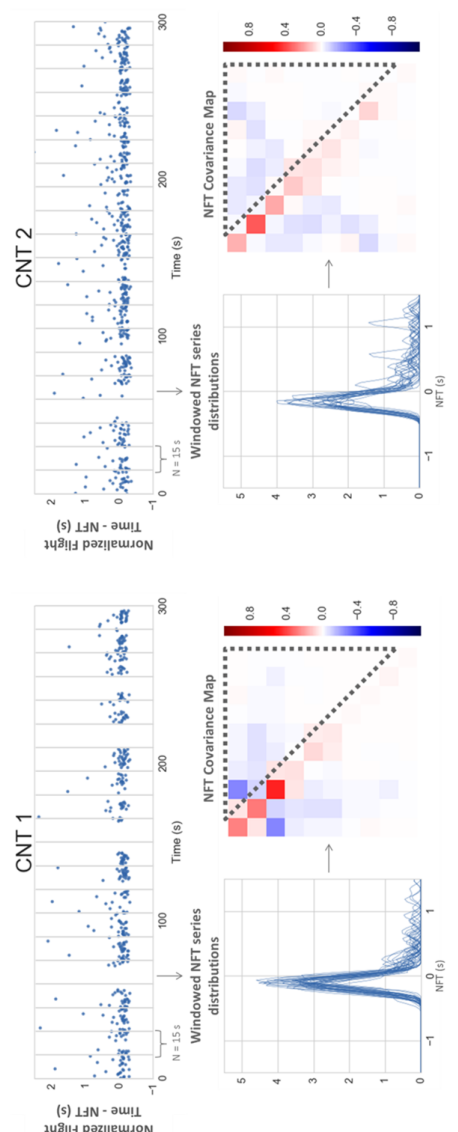


\section{Evaluation Methodology}

The proposed features are based on a limited set of parameters that are estimated on an external dataset of 27 healthy subjects. These parameters, shown in the previous sections, provide a general description of the typing signal and are independent of motor function status, i.e. they are not optimized to enhance the separation between Parkinson's participants and controls.

First, we assess the classification performance of the proposed typing features with univariate methods. Next, we evaluate the joint discriminatory power of the features in a multivariate analysis framework.

The multivariate method assembles a feature selection transform followed by a final estimator. We use a nested-cross validation strategy for performance evaluation, i.e. a combination of two embedded cross-validation loops. The inner kfold cross-validation loop is used to identify the relevant features and estimate the model hyperparameters based on the training folds of the outer leave-one-out crossvalidation fold. The outer loop is run using the best model settings estimated in the inner loop and storing the score for the left-out sample.

We used two tests to evaluate the ability of each metric, typing features and models' scores, to correctly separate the referred classes: the Receiver Operating Characteristic (ROC) analysis and the Mann-Whitney $U$ test to reject the null hypothesis that the controls and the Parkinson's samples come from the same distribution.

The ROC analysis consists of an iterative process that monotonically increases the value of the metric under study to define a dynamic threshold. On each iteration the current threshold value is evaluated as a binary classifier that separates Parkinson's and controls. The output is a set of sensitivity/(1-specificity) pairs that are joined to draw the corresponding ROC curve. The Area Under the Curve (AUC) can be interpreted as the probability that the classifier will rank a randomly chosen positive instance higher than a randomly chosen negative one. Moreover, this metric allows a reliable comparison of the performance even when the number of cases and controls is not fully balanced, as it is the case of our study dataset (48\% PD, 52\% CNT). A sampling with replacement method (1,000 bootstraps) defines a ROC distribution from which we compute the average and confidence intervals of the AUC values to describe the classification performance of each metric.

\subsection{Results}

Table 5.2 shows the results obtained for the univariate feature evaluation. The values presented for each typing metric include: mean value and standard deviation grouped by condition, average AUC for the bootstrapped ROC distribution, AUC confidence interval computed as the $\left[5^{\text {th }}, 95^{\text {th }}\right]$ percentiles on the resulting AUC values, and the Mann-Whitney significance test outcome.

Table 5.3 summarizes the results of the multivariate analysis. We evaluate nine different models defined as the possible combinations of three feature selectors and three classifiers. These methods were selected to represent different families of common machine learning approaches. Feature ranking methods used are Lasso, Ridge regression and Gini impurity based random forests used as estimators in a recursive feature elimination framework. Classification methods considered are logistic regression, linear kernel Support Vector Machines (SVM) and AdaBoost.

Alternating finger-tapping (AFT), a quantitative upper limb motor test commonly used in clinical trials to monitor PD signs, is used as the reference metric to evaluate the performance of the proposed method. Also, we include the average of the 
TABLE 5.2: Results Univariate Analysis (Independent typing features). The table shows the mean values and performance of the typing features and the reference metrics, including the ROC AUC mean and confidence intervals achieved by each measurement and the results of the Mann-Whitney $U$ test to analyze if the null hypothesis, that Parkinson's disease (PD) and control (CNT) subjects come from the same population, can be rejected. Covariance sum $\sum|\mathrm{Cv}|$ presents the best discrimination performance with an AUC of 0.91 and significance $\mathrm{p}<0.001$.

\begin{tabular}{ccccl}
\hline Feature & $\begin{array}{c}\text { Avg. (std) } \\
\text { Parkinson's }\end{array}$ & $\begin{array}{c}\text { Avg. (std) } \\
\text { Controls }\end{array}$ & AUC [5\%,95\%] & Significance \\
\hline \hline$\overline{S k}$ & $0.955(0.572)$ & $1.742(0.536)$ & $0.85[0.74,0.95]$ & $\mathrm{p}<0.001$ \\
\hline$\sigma_{S k}$ & $0.652(0.135)$ & $0.837(0.210)$ & $0.77[0.64,0.87]$ & $\mathrm{p}<0.01$ \\
\hline $\bar{K} t$ & $0.767(1.749)$ & $3.587(2.594)$ & $0.87[0.78,0.95]$ & $\mathrm{p}<0.001$ \\
\hline$\sigma_{K t}$ & $1.691(0.977)$ & $3.595(1.593)$ & $0.88[0.78,0.95]$ & $\mathrm{p}<0.001$ \\
\hline$\overline{C v}$ & $-0.019(0.015)$ & $-0.018(0.006)$ & $0.46[0.38,0.69]$ & $\mathrm{p}=0.66$ \\
\hline$\sigma_{C v}$ & $0.104(0.030)$ & $0.068(0.029)$ & $0.84[0.72,0.93]$ & $\mathrm{p}<0.001$ \\
\hline$\sum|C v|$ & $3.290(1.071)$ & $1.839(0.656)$ & $0.91[0.82,0.97]$ & $\mathrm{p}<0.001$ \\
\hline \hline $\mathrm{n}($ total $\mathrm{n}=44)$ & 21 & 23 & & \\
\hline \hline
\end{tabular}

TABLE 5.3: Results Multivariate Analysis (Aggregated typing features). The table summarizes the results of the multivariate analysis. We evaluate the classification performance of different models that aggregate the information of the proposed typing features. We tested a total of nine models, built as the possible combinations of three different feature selection methods and three estimators. A nested cross validation framework was implemented to train and test the models. For each model, we include the mean and confidence intervals of the AUC and the results of the Mann-Whitney $U$ test to reject the null hypothesis that Parkinson's disease (PD) and control (CNT) subjects come from the same population. Model 2, a linear support vector classifier preceded by L1-regularized linear model (Lasso) for feature selection, presents the best discrimination performance with an AUC of 0.88 and significance $\mathrm{p}<0.001$.

\begin{tabular}{ccccccc}
\hline & & & Avg. Score $(\mathrm{std})$ & Avg. Score $(\mathrm{std})$ \\
Model & Feature Selection & Classifier & Parkinson's & Controls & AUC [5\%,95\%] & Significance \\
\hline \hline 1 & L1 (Lasso) & Logistic Regression & $2.127(2.883)$ & $-1.684(2.127)$ & $0.87[0.75,0.94]$ & $\mathrm{p}<0.001$ \\
\hline 2 & L1 (Lasso) & Linear SVM & $0.825(1.109)$ & $-0.663(0.730)$ & $0.88[0.78,0.95]$ & $\mathrm{p}<0.001$ \\
\hline 3 & L1 (Lasso) & AdaBoost & $3.603(6.612)$ & $-0.218(1.591)$ & $0.73[0.58,0.84]$ & $\mathrm{p}<0.01$ \\
\hline 4 & L2 (Ridge) & Logistic Regression & $1.767(2.870)$ & $-2.241(2.293)$ & $0.87[0.77,0.95]$ & $\mathrm{p}<0.001$ \\
\hline 5 & L2 (Ridge) & Linear SVM & $0.736(1.063)$ & $-0.709(0.926)$ & $0.86[0.74,0.95]$ & $\mathrm{p}<0.001$ \\
\hline 6 & L2 (Ridge) & AdaBoost & $0.617(1.599)$ & $-1.464(1.734)$ & $0.82[0.69,0.92]$ & $\mathrm{p}<0.001$ \\
\hline 7 & Gini Impurity & Logistic Regression & $1.434(2.350)$ & $-1.236(1.850)$ & $0.81[0.67,0.91]$ & $\mathrm{p}<0.001$ \\
\hline 8 & Gini Impurity & Linear SVM & $0.650(1.035)$ & $-0.588(0.683)$ & $0.86[0.74,0.95]$ & $\mathrm{p}<0.001$ \\
\hline 9 & Gini Impurity & AdaBoost & $2.131(5.123)$ & $-1.019(1.972)$ & $0.80[0.66,0.90]$ & $\mathrm{p}<0.05$ \\
\hline \hline $\mathrm{n}$ (total $\mathrm{n}=44)$ & 21 & 23 & & & & \\
\hline \hline
\end{tabular}


TABLE 5.4: Methods Comparison. The table compares the performance of the touchscreen typing based metrics, including the raw flight time average $(\bar{X})$ and best performing univariate $\left(\sum|C v|\right)$ and multivariate methods (Model2), with the alternating finger-tapping test AFT. The presented methods improve the discrimination ability of the reference test (AFT: 0.85[0.76, 0.94] AUC and 0.75/0.78 sensitivity/specificity), with 0.91[0.82, 0.97] AUC and 0.81/0.81 sensitivity / specificity for the best performing feature $\left(\sum|\mathrm{Cv}|\right)$ and $0.88[0.78$, 0.95] AUC and $0.73 / 0.84$ sensitivity/specificity for the best multivariate model (Model2). The adequacy of the proposed methods to enhance the differences of the typing patterns between Parkinson's subjects and controls is stressed by the comparison with the raw signal based metric ( $\bar{X}: 0.83[0.71,0.91]$ AUC and $0.73 / 0.72$ sensitivity/specificity). The presented sensitivity/specificity pairs correspond to the closest-to- $(0,1)$ cut-off point. The unadjusted statistical significance is computed with two-sided Mann-Whitney $\mathrm{U}$ test. The adjusted significance tests were computed with logistic regression models including gender and age as co-variates. For the developed methods none of the co-variates reached statistical significance.

\begin{tabular}{cccccccc}
\hline & $\begin{array}{c}\text { Avg. }(\mathrm{std}) \\
\text { Parkinson's }\end{array}$ & $\begin{array}{c}\text { Avg. }(\mathrm{std}) \\
\text { Control }\end{array}$ & AUC $[5 \%, 95 \%]$ & Specificity & Sensitivity & $\begin{array}{c}\text { Significance } \\
\text { Adjusted }\end{array}$ & $\begin{array}{c}\text { Significance } \\
\text { Unadjusted }\end{array}$ \\
\hline \hline Best Univariate $\left(\sum|C v|\right)$ & $3.290(1.071)$ & $1.839(0.656)$ & $0.91[0.82,0.97]$ & 0.81 & 0.81 & $\mathrm{p}<0.001$ & $\mathrm{p}=0.002$ \\
\hline Best Multivariate $($ Model 2$)$ & $0.825(1.109)$ & $-0.663(0.730)$ & $0.88[0.78,0.95]$ & 0.84 & 0.73 & $\mathrm{p}<0.001$ & $\mathrm{p}=0.002$ \\
\hline Raw Flight Time Average $(\bar{X})(\mathrm{s})$ & $0.870(0.283)$ & $0.566(0.155)$ & $0.83[0.71,0.91]$ & 0.72 & 0.73 & $\mathrm{p}<0.001$ & $\mathrm{p}=0.003$ \\
\hline Alternating finger-tapping $(A F T)$ & $49.17(10.65)$ & $67.54(14.11)$ & $0.85[0.76,0.94]$ & 0.78 & 0.75 & $\mathrm{p}<0.001$ & $\mathrm{p}=0.002$ \\
\hline
\end{tabular}

unprocessed flight time signal $(\bar{X})$ as a starting point to show the improvement introduced by our solution to the discrimination ability measured on the raw typing data. In this case, we did not use any machine learning model, but rather evaluated the discriminative ability of the variable by directly testing all possible thresholds. We replicate the evaluation framework used in our methods to test the classification performance of these two reference metrics in our cohort.

Figure 5.4 and Table 5.4 show the performance comparison of the touchscreen typing based metrics, i.e. raw flight time average and the developed methods, with the AFT test reference. Raw flight time average $(\bar{X})$ presents an AUC of 0.83 [0.710.91]. The best performing typing feature, covariance sum $\left(\sum|C v|\right)$, presents an AUC of 0.91 [0.82-0.97]. The best multivariate method (Model2), a combination of L1regularized feature selection plus a linear SVM as the final estimator, scores an AUC of 0.88 [0.78-0.95]. AFT test performance measured in our cohort achieves an AUC of 0.85 [0.76-0.94]. Sensitivity and specificity values shown in Table 5.4 are estimated using the closest-to- $(0,1)$ criterion to define the cut-off point Perkins and Schisterman, 2006. Unadjusted p-values present the results of the Mann-Whitney $U$ test to reject the null hypothesis that PD and CNT subjects come from the same population. Adjusted significance tests the null hypothesis that the metric under scrutiny does not contribute to the separation between PD and control groups in a logistic regression model accounting for sex and age.

Finally, we evaluated the classification performance of the proposed methods for different signal lengths, in order to analyze the appropriate duration of continuous typing that would be necessary to achieve significant results. In Figure 5.5 we illustrate the results of this analysis for our best univariate and multivariate methods. 
FIGURE 5.4: PD versus Control, ROC comparison. Comparison of receiver operating characteristic (ROC) curves showing the classification rate for the typing based metrics, including raw flight time average $(\bar{X})$ and best performing univariate $\left(\sum|C v|\right)$ and multivariate methods (Model2), with the alternating finger-tapping test AFT. Statistical significance of the Mann-Whitney U test is estimated to reject the null hypothesis that the two groups, PD and CNT, come from the same population. Statistical significance noted as: $\mathrm{p}<0.001\left({ }^{* *}\right)$, $\mathrm{p}<0.01\left(^{* *}\right)$ and $\mathrm{p}<0.05\left(^{*}\right)$.

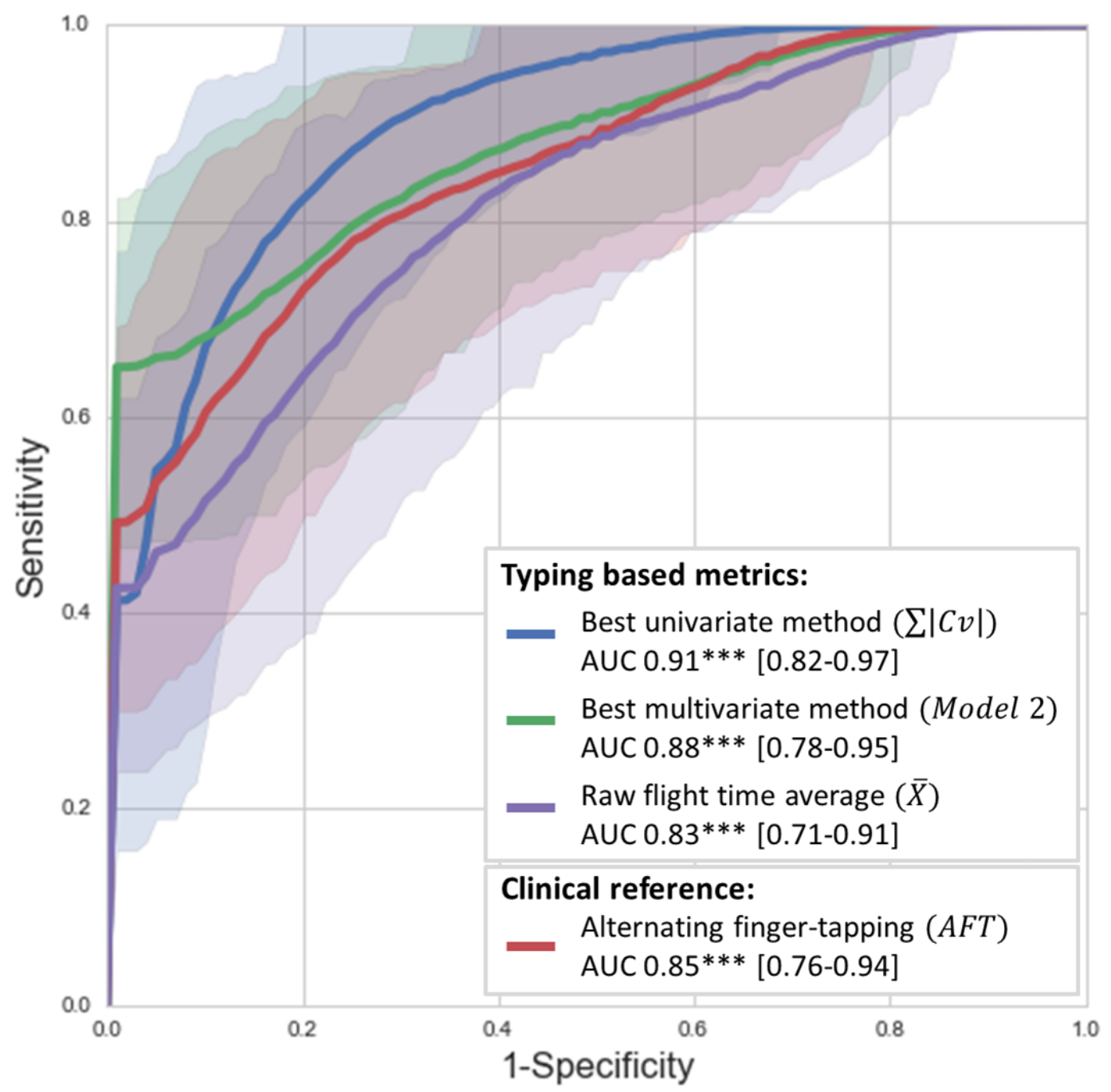


FIGURE 5.5: Classification performance as a function of typing time. The figure shows the evolution of the area under the ROC curve (AUC) for the best performing feature $\left(\sum|C v|\right)$ and best multivariate model (Model2) as we increment the amount of typing data used to perform the analysis. We observe a clear improvement of the classification performance as the duration of the analyzed typing series increases.

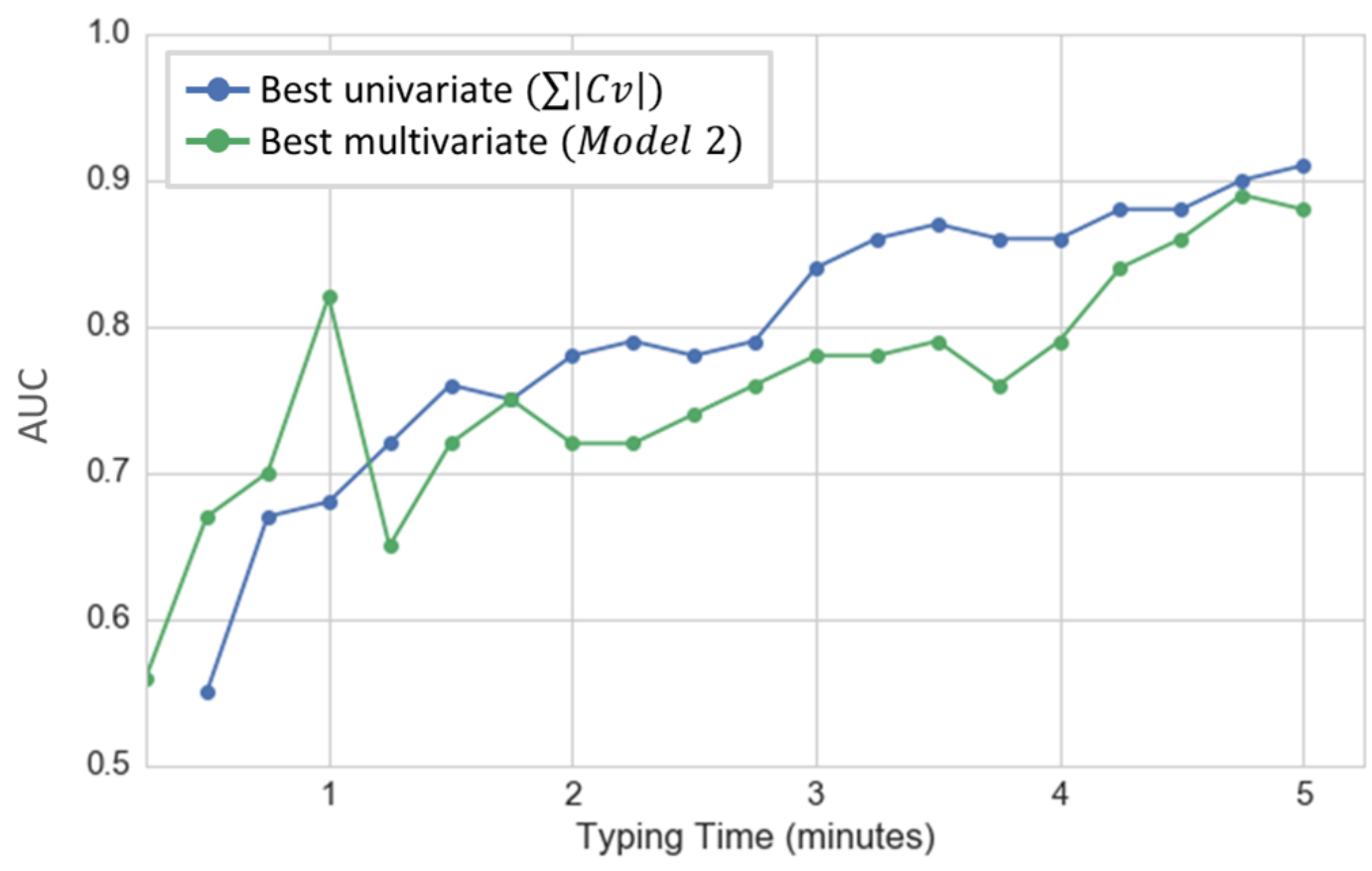




\subsection{Discussion}

In this chapter we present an algorithm to identify PD motor signs by analyzing the typing activity on smartphones independently of the typed text. Users do not need to wear any sensor or remember to perform a structured test. Compliance depends only on the act of installing the software. Once installed, data collection happens automatically without interfering with the normal use of the device.

The current clinical standard used to quantify PD stage and progress present some limitations that define a clear need in the treatment and control of the disease. This scenario has led to the study and development of different alternatives in attempting to complete and complement UPDRS information.

In our cohort, a commonly used quantitative method that evaluates upper limbs dexterity, alternating finger-tapping (AFT), discriminated both populations with an AUC of 0.85 with $0.75 / 0.78$ sensitivity/specificity. The proposed methods outperform this clinical reference, achieving an AUC of 0.91 with $0.81 / 0.81$ for the best performing typing feature, the covariance sum $\left(\sum|\mathrm{Cv}|\right)$, and an AUC of 0.88 with $0.73 / 0.84$ for the best multivariate method, a pipeline comprised of L1-regularized feature selection and a linear SVM as the final classifier.

We believe that our approach is able to achieve such performance because of bradykinesia, bimanual coordination problems and other PD signs that may alter typing kinetics in a way detectable through a keystroke timing data analysis. PD motor impairment, in the particular case of the FT signal, may impede PD patients to press and release the keys in a consistent manner, which we hypothesize would induce irregular flight times (similar to what may be seen in finger tapping tests). Our results are consistent with that hypothesis in that the typing signal distribution for PD patients has a greater dispersion and temporal variability, i.e. the heteroskedasticity measured using those features. The improvement achieved in the classification rate, compared to the alternating finger-tapping test, may be due to the fact that our features (i.e. skewness, kurtosis and covariance of the FT distribution) have been carefully defined to specifically capture these motor abnormalities that are a direct representation of PD signs. The approach of constructing the typing signal as a sequence of consecutive signal segments allows an intrasubject analysis, which optimizes the detection of the internal variability introduced by PD signs.

One of the main difficulties when using the typing signal as the unique source of information is the risk of measuring variables that are representative of the typing style but do not capture the effect of PD signs. We limit this effect by applying a normalization phase that forces a zero mean. This focuses the analysis on the FT distribution shape and variability. Another external factor that has to be taken into account when studying the potential limitations of the proposed method is the requirement of a minimal number of signal samples to make the sub-distribution analysis consistent. To collect enough information from the natural typing signal, a minimum level of skills in touchscreen typing is demanded in order to meet the established criteria. We consider that, taking into account the rapid growing rate of smartphone users, typing skills will not limit the application of this method.

Our methods were validated in a controlled environment. Participants were asked to type for 5 minutes to complete the touchscreen test. Although they were instructed to type as they would normally do in order to reflect actual routine use of the device, further analysis will be necessary to discard a significant influence of the controlled test on their typing behavior. Regarding the 5-minute duration of the test, we understand that not all the smartphone users are likely to continuously type for this amount of time, however, the proposed methodology can be applied on natural 
typing signals collected for longer periods of time whose aggregate active typing time is 5 minutes or more.

This study is a step towards the final goal of developing an automated biometric tool for diagnostic and therapeutic decision support in PD. The presented methodology compares well to standard clinically-used methods in terms of its ability to differentiate PD participants from controls, and is able to do so from information collected from touchscreen typing activity. In our cohort, PD population presented mild signs (average UPDRS-III score of $17.76 \pm 7.92$ and range [6, 41]), this suggests that the proposed features are able to discriminate PD from controls even at early disease stages. However, as a pilot study, the findings of this research must be considered with caution. A further validation of this methodology would require a larger and better balanced cohort that enables a comprehensive review of the influence of the potential confounding variables mentioned in this work and others, such as medication state and cognitive deficits. Future work will include new studies to collect subjects' daily interaction with their smartphones in order to validate the applicability of the presented methods in a passively-monitored environment. Additional information from user's daily interaction with smartphones, such as pressure, gesture typing and accelerometer data, could be used to complement our keystroke based analysis. Method functionality could also be improved with the appropriate algorithms. Turning from a classification to a regression model, it may be possible to quantify a continuous metric for the natural progression of the disease over continuous motor function evaluations. 


\section{Chapter 6}

\section{Response to medication}

Today, no cure has been discovered for Parkinson's disease (PD). Available medication can only provide symptomatic relief. The most commonly prescribed drug for PD is Levodopa (L-dopa), which helps control some of the motor symptoms, such as bradykinesia, stiffness and rigidity, but does not protect against disease progression. Levodopa works by replacing the chemical messenger dopamine, which the brain cells in the substantia nigra are no longer producing (Haddad et al., 2017). Chronic use of L-dopa has been associated with a range of adverse effects. After five years the majority of these patients suffer fluctuations, dyskinesias, toxicity, or loss of efficacy (Marsden, 1994). Current therapeutic strategies focus on delaying the long-term complications of treatment and maximize symptomatic relief. Decision making needs to take into account individuals' circumstances and requirements of the patient to balance tolerance for adverse effects with the amount of symptomatic relief required (Ferreira et al., 2013).

Current standards for the assessment of PD, such as the Unified Parkinson's Disease Rating Scale (UPDRS), present limitations that constraint physicians' ability to evaluate the adequacy of titration parameters and adjust it to individual patients' need, taking into account their particular functional disabilities and specific handicaps. These scales report a semiquantitative and subjective score, non-sensitive to subtle motor changes (Kieburtz and Olanow, 2015; Schneider et al., 2012; Maetzler et al., 2013b). In addition, these assessments typically require the patient to travel to the clinic, and they need to be performed by a trained specialist, representing an additional burden for the patient and hence being time and cost consuming.

For these reasons, several attempts are underway to complement traditional standards with more objective, quantitative, and continuous outcome measures (SánchezFerro et al., 2016b; Ferreira et al., 2015).

The increasing digitalization of large volumes of patient generated data and recent advancements in technologies to analyze this massive amount of information are accelerating the introduction of the concept of real-world evidence in healthcare. This new concept has the potential to disrupt not only PD management but also drug discovery. Since randomized clinical trials, the current standard in drug development, have limitations to provide a complete representation of the actual outcomes of new therapies, real world data is becoming progressively important to help provide broader scientific evidence and a better understanding of new drugs' effectiveness and safety.

Building on previously presented results, where we showed how motor signs of neurodegeneration in early Parkinson's disease can be detected through the analysis of the patterns derived from fingers interaction with mechanical and touchscreen keyboards, our objective is to evaluate the ability of the proposed approach to detect longitudinal changes in patients' motor performance induced by dopaminergic therapies. 
TABLE 6.1: Demographic and baseline characteristics The table includes a comparison of the clinical and demographic variables between the PD and control groups.

\begin{tabular}{lccc}
\hline Variable & Parkinson's $(\mathrm{n}=31)$ & Healthy controls $(\mathrm{n}=30)$ & $\mathrm{P}$-value \\
\hline \hline UPDRS-III, median (IQR) & $10[17,26]$ & $1[0,2]$ & $\mathrm{p}<0.001$ \\
\hline Age in years, median (IQR) & $59.78[54.19,68.60]$ & $63.0056 .48,69.44]$ & 0.476 \\
\hline Number of women, $\mathrm{n}(\%)$ & $14(48.3)$ & $16(53.3)$ & $\mathrm{p}=0.797$ \\
\hline \hline
\end{tabular}

In this chapter we present preliminary results supporting the validity of the proposed methodology to generate typing-derived measures that are able to detect the impact of medication response in PD, bringing us a step closer to the development of a quantitative metric that could be used as a surrogate marker of drug response in clinical trials. A clinical evaluation of these results was published in "Remote Monitoring of Treatment Response in Parkinson's Disease: The Habit of Typing on a Computer" (Matarazzo et al., 2019). Here, we will deepen in the technical aspects of the proposed methodology.

\subsection{Methods}

We present the results of a 6-month longitudinal study designed to evaluate drugresponsiveness in PD. A total of 31 early PD patients and 30 age- and sex-matched controls (CNT) were recruited from 7 hospitals in Madrid, Spain. Inclusion criteria for the PD group were: (i) diagnosis of Parkinson's disease according to the UK Brain Bank criteria 1; (ii) younger than 85 years of age; (iii) motor symptoms for less than 5 years before enrollment; (iv) new indication of dopaminergic treatment; and (v) an average self-reported computer use of at least 30 minutes per day. The exclusion criteria were (i) the presence of cognitive impairment according to DSMIV criteria for dementia (Erkinjuntti et al., 1997); (ii) relevant hand deformities (e.g. severe osteoarthritis) or (iii) other neurological motor conditions. Controls were enrolled after ruling out the existence of parkinsonism, hand deformities, cognitive impairment, sleep problems, or any other potential confounders (e.g. use of psychoactive medication, drug abuse, or a serious medical condition). All of the experimental protocols were approved by the Massachusetts Institute of Technology (no. 1412006804), HM Puerta del Sur University Hospital, Spain (no. 15. 05.796-GHM), 12 de Octubre University Hospital, Spain (no. CEIC:14/090), and Hospital Clínico San Carlos University, Spain (no.14/136-E). All of the participants provided written informed consent prior to study enrollment (Table 6.1).

\subsubsection{Study design}

A schema of the study design is presented in Figure 6.1. Participants received a complete evaluation carried out by a movement disorder specialist during the baseline visits and at weeks 4, 8, 16 and 24 from the enrollment date. The assessment included gathering of basic socio-demographic information and a standardized motor evaluation (Unified Parkinson's disease rating scale part III (UPDRS-III) (Goetz et al., 2008), Purdue pegboard test (Tiffin and Asher, 1948) and Alternating finger-tapping (AFT) (Homann et al., 2000). To be able to adjust for potential confounding effects of cognition on typing performance, both the PD and healthy controls were evaluated by a neuropsychologist with specific training in movement disorders. These evaluations 
FIGURE 6.1: Schema of study design. A six-month naturalistic study including a group of 31 early PD patients and 30 matched controls. On the baseline visit, participants where asked to bring their personal laptop to get the neuroQWERTY software installed. The software ran in the background of participants' machine collecting any keystroke data generated during the follow-up period. Over the duration of the study, five on-site evaluations were scheduled. Each visit included a clinical evaluation and an on-site controlled typing task on an standard device. Participants in the PD group were de-novo when recruited for the study. They remained untreated for at least a week from their baseline visit. After that, medication and changes in titration parameters were tracked over the follow-up period.



were made according to recent recommendations of the MDS-task force for the diagnosis of cognitive impairment in PD (Goetz et al., 2008). Prescription schemes were verified with the referring neurologist and the participants throughout the whole study duration.

At the baseline visit, the neuroQWERTY software was installed in participants' personal computers. If they shared their computer, we provided them with a laptop with pre-installed software. Participants were encouraged to freely type for at least 15 minutes per day during the whole duration of the study but otherwise use the computer as they would do normally. The software ran in the background of the device, capturing the timing information of press/release keystroke events thorough the follow-up period. Additionally, every in-clinic visit included a controlled typing task where participants were asked to transcribe an non-standardized sample text on a standard word processor for 15 minutes. To emulate natural interaction with the device, subjects were asked to type as they normally would do as part of their daily routine. This controlled test was performed using a standard machine, specifically a Lenovo G50-70 i3-4005U with 4GB of memory and a 15 inches screen running Manjaro Linux operative system.

The sample size was predefined to detect at least 15 participants with response to medication. Power analyses where completed based on a previous definition of response that established a decrease of at least five points in the UPDRS-III score as a clinically relevant change (Schrag et al., 2006). The a priori responder rate estimate was $50 \%$, based on the results published from several randomized clinical trials (Jankovic et al., 2007; Poewe et al., 2011; Kieburtz and Parkinson Study Group 
PramiBID Investigators, 2011).

In the PD group, we targeted untreated de-novo patients who where ready to start dopamine agonist or levodopa therapy. To assure enough at-home off-medication typing data, PD participants were instructed to delay the start the newly prescribed treatment for a seven-day period after the initial baseline visit. Further follow-up visits were scheduled flexibly on weeks $4,8,16$, and 24 from the date of enrollment. Six of the PD participants that were already on rasagiline where not excluded from the study as the expected confounding motor effect was not significant according to published results on the main pivotal trials that reported a change $\leq 0.11$ in the UPDRS in a four to six-month period (Olanow et al., 2009). The remaining 24 PD patients were completely drug naïve at baseline.

\subsubsection{Motor change definition}

A common limitation in the study of drug response is the misalignment between statistical and clinical significance of the changes induced by therapy. To solve this inconsistency, it is necessary to define the smallest difference in a rating scale that is perceived by patients as beneficial or harmful and which would lead the clinician to consider a meaningful change in treatment (Revicki et al., 2008). This concept was introduced in medical research as Minimally Clinical Important Change (MCIC).

Several methods have been proposed for determining the MCIC of a scale. Among them we can define two main categories, anchor-based and distribution-based methods (Wright et al., 2012):

1. Anchor-based methods use an external variable, or anchor, to classify the subjects and, based on that classification, define the cutoff of the scale under study using metrics of central tendency (i.e median/mean) or receiver operating curve analyses (ROC) on the subjects that changed. The anchor variable used to define the change is mostly a patient-reported outcome (health outcome directly reported by the patient who experienced it) or a clinical-reported outcome (based on the clinical impression of the healthcare professional).

2. Distribution-based methods are based on the statistical characteristics of the variable of interest. They evaluate the probability that the change occurred by chance and hence use measures of dispersion. The most commonly used ones are the Standard Error of Measurement (SEM) and the Stardard error of the difference $\left(S_{\text {diff }}\right)$. The main limitation of the distribution-based approaches is that they do not take into account any additional clinical input. Thus, they may not be able to detect a change that could be important from a clinical standpoint. Yet, they represent a useful approximation to avoid changes that could be attributed to the scale intrinsic noise.

Since the Unified Parkinson's Disease Rating Scale (UPDRS), and specifically the motor section or part III, is the most used tool to assess motor change in PD, we relied on this sub-scale to define the cutoff that separates responders from non-responders in the context of this longitudinal study (Sánchez-Ferro et al., 2018).

To guide the validation of the proposed method we pre-specified two cut-offs based on previous observations (Schrag et al., 2006; Shulman et al., 2010). In order to confirm which one was applicable to a naturalistic setting, we combined distribution- and anchor-based methods to define the MCID at the final visit.

For the distribution based approach, we employed the $S_{\text {diff }}$ to define the minimum change in the scale not occurred by chance. The $S_{\text {diff }}$ was calculated using the $S E M$ of the baseline and the final visits (week 24) with the following formula: 


$$
S_{\text {diff }}=\sqrt{\left(S E M_{B}\right)^{2}+\left(S E M_{W_{24}}\right)^{2}}
$$

For the anchor-based approach we employed three different external variables:

1. The Physical Health item of the Satisfaction with Life Scale (SLS-6). The SLS6 scale is rated by the patient and this specific item evaluates the patient's physical health ranging from 0 (worse) to 10 (better).

2. The Disability item of the Clinical Impression of Severity Index (CISI-PD). The CISI-PD scale is rated by the health-care professional and this specific item evaluates the disability related to PD in the patient. It ranges from 0 (better) to 6 (worse).

3. The Motor Signs item of the Clinical Impression of Severity Index (CISI-PD). Also rated by the health-care professional and the specific item evaluates the parkinsonian motor signs in the patient from 0 (better) to 6 (worse).

For the three anchors, one point or greater variation towards getting better or worse was considered relevant to define a significant change. We applied ROC analysis to define the best UPDRS-III cutoff point to separate change versus no-change as defined by each of the selected anchors. The cutoff point was estimated using the closest-to-(0,1), i.e. the use case that maximizes the sensitivity/specificity pair (Perkins and Schisterman, 2006). A unique anchor-based threshold was defined by triangulating the three resulting cutoffs for each of the anchor variables.

The final MCIC used as a reference in this study was defined by selecting the most conservative cutoff obtained from each of the methods. This resulted in a fivepoint decrease threshold in the UPDRS-III scale, measured in the final visit relative to the baseline visit, to separate PD responders from non-responders. Participants that presented and increase above the change threshold (over five points in the UPDRSIII scale), i.e. those whose motor performance worsened significantly during the follow-up period, where not considered for the analysis.

\subsubsection{Data availability and group distribution}

In order to limit the effect of noisy typing patterns, we empirically defined thresholds for "active typing days" which will be used for our subsequent analyses. An active typing day was defined by at least 10 valid windows within a day, where a valid window was represented by a data sequence of at least 30 keystrokes in 90second time interval. To ensure a complete longitudinal representation of the typing patterns over the analysis period, a 15-day rolling window was applied to identify gaps in computer activity. Only participants with one or more active days in at least $80 \%$ of every possible sliding window within the six-month follow-up were considered for the analysis.

Out of the 60 study participants, two PD patients and one healthy control did not complete the follow-up. After applying our MCIC definition on the remaining 28 Parkinson's subjects, the group change distribution resulted in 14 PD responders, 9 PD non-responders and 5 PD patients that got worse. Since the proposed approach was designed with the purpose of characterizing positive response to medication, the subset of participants that worsened was excluded from the analysis.

Finally, 15 additional participants were discarded for not reaching the minimum threshold established for longitudinal typing activity. The final group distribution 
of participants included in the analysis, after excluding non-consistent typers was: 20 healthy controls, 6 PD non-responders and 11 PD responders (see Figure 6.2 for detail).

\subsubsection{Analysis of in-clinic data}

Through the 6-month follow-up period participants were clinically evaluated at Baseline and Weeks 4-8-16-24 since they enrolled the study. Each in-clinic visit included a motor examination and a controlled typing task. We evaluated variations in the keystroke distributions registered for each of the in-clinic visits in order to understand the relationship between the observed motor change and the statistical distance measured between pairs of typing events. Specifically, we looked into the variations of the distributions of hold times (HT), i.e. the sequence of values that measure the time between pressing and releasing a key.

\section{Signal representation}

Let the vector $A_{1}, A_{2}, \ldots, A_{N}$ represent a sequence of key hold times recorded within a given typing session. We consider only the keys for which we expect a short hold time, i.e. alphanumeric characters, symbols and space bar.

We apply kernel density estimation (KDE) (Parzen, 2007), a non-parametric method to estimate the probability density function of a random variable, to generate and estimate of the underlying probability density function $\left(\rho_{K, A}\right)$ of $A$ :

$$
\rho_{K, A}(y)=\sum_{i=1}^{N} K\left(\left(y-A_{i}\right) / h\right)
$$

where $N$ is the number of keystrokes that conform the typing sample, $K$ is a smooth function called the kernel function and $h>0$ is the smoothing bandwidth that controls the amount of smoothing of the resulting distribution. Here we used a Gaussian kernel that can be formally expressed as follows:

$$
K(x: h) \propto \exp \left(-\frac{x^{2}}{2 h^{2}}\right)
$$

The kernel bandwidth parameter $h$ was estimated in a patient level by applying the Sheather \& Jones method for data-based bandwidth selection using the data from the controlled typing task collected during the baseline visit (Sheather and Jones, 1991).

\section{Univariate Analysis}

To analyze the ability of the controlled in-clinic typing tasks to provide an objective representation of the motor change induced by medication, we applied statistical distance analysis to generate a series of metrics that quantify changes in the shape, symmetry and absolute value of the HT probability density functions estimated at different time points during the follow-up. Specifically we evaluated the variation in the distributions measured between week $8\left(\rho_{K, A_{W_{8}}}\right)$ and week $24\left(\rho_{K, A_{W_{24}}}\right)$, relative to the reference HT distribution measured at baseline $\left(\rho_{K, A_{B}}\right)$.

The following univariate metrics where used for the in-clinic data analysis:

1. Correlation-based $\left(\operatorname{Corr}_{B, W_{T}}\right)$ : identifies the maximum value of the cross-correlation measured between the baseline HT distribution $\left(\rho_{K, A_{B}}\right)$ and the distribution estimated $T$ weeks after the baseline visit $\left(\rho_{K, A_{W_{T}}}\right)$. 
FIGURE 6.2: Typing activity map. A representation of the typing activity gathered during the study. The figure presents two heatmaps, left for the PD group and right for the CNT group. Each row in the heatmap provides a sequential representation of each of the participants' typing activity over the 180-day study period. The darker color marks represent the days corresponding to their study visits. The colors in the PD heatmap represent the patients' status with regard to medication response at the end of the study. The blanks represent inactive days. An active typing day was defined by at least 10 valid windows within a day, where a valid window was represented by a data sequence of at least 30 keystrokes in 90-second time interval. To ensure a complete longitudinal representation of the typing patterns over the analysis period, a 15-day rolling window was applied to identify gaps in computer activity. Only participants with one or more active days in at least $80 \%$ of every possible sliding window within the six-month follow-up were considered for the analysis.

People with Parkinson's (PD)

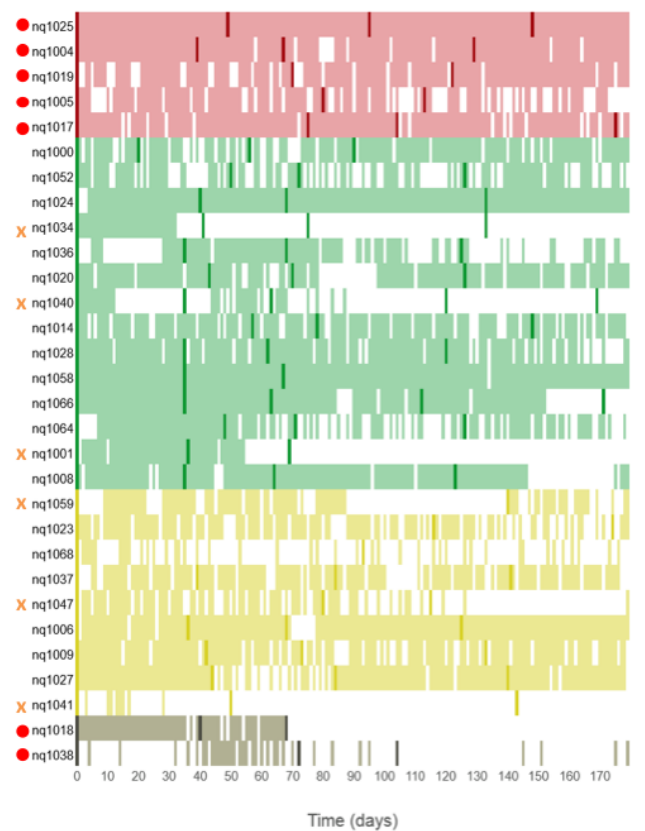

Time (days)

| $\mathrm{PD}$ - Worsen (5)

I PD - Responder (14) I Control (29)

I Did not complete follow-up (3)
Healthy Controls (CNT)

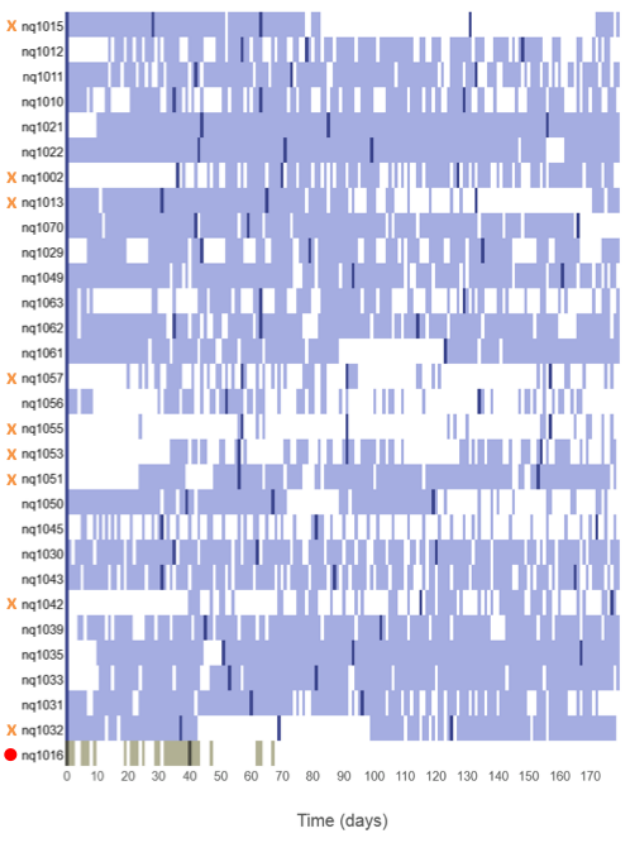

Light color - Meaningful typing activity on date X (>30 keystrokes) Dark color - In clinic visit on date $X$

- Not included in the analysis $x$ Not enough typing data 
2. Convolution-based $\left(\operatorname{Conv}_{B, W_{T}}\right)$ : identifies the maximum value of the discrete convolution operation applied to the baseline HT distribution $\left(\rho_{K, A_{B}}\right)$ and the distribution estimated $T$ weeks after the baseline visit $\left(\rho_{K, A_{W_{T}}}\right)$.

3. Bhattachayyara-based $\left(\right.$ Bhat $\left._{B, W_{T}}\right)$ : measures the similarity between the baseline HT distribution $\left(\rho_{K, A_{B}}\right)$ and the distribution estimated $T$ weeks after the baseline visit $\left(\rho_{K, A_{W_{T}}}\right)$.

\subsubsection{Analysis of At-Home Data}

As opposed to the episodic nature of in-clinic assessments, at home data collection provides an opportunity to use algorithms suited to monitoring changes over time. We designed a neural network architecture based on a mixture of Long-Short Term Memories (LSTM) (Hochreiter and Schmidhuber, 1997) and dense layers with the objective of capturing long term time relations in the typing patterns.

\section{Signal representation}

Let the structure $X[T]$ define the pre-processed input data generated from an athome keystroke data stream, being $T$ an integer representing the number of weeks from the baseline visit used to compute the signal input, a given sequence of key hold times is represented as follows (Figure 6.3):

$$
X[T]=\left\{X_{1}[T], X_{2}[T]\right\}
$$

$X_{1}[T]$ is a static feature vector based on the cross-sectional algorithm presented in Chapter 4:

$$
X_{1}[T]=M_{t=1}^{T}\left(x_{t}\right)
$$

where $M_{t=1}^{T}$ denotes the median of the $x_{t}$ nQi-based weekly feature vectors from baseline $(t=1)$ till week $T$ :

$$
x_{t}=\left[A_{t}^{\text {out }}, A_{t}^{\text {iqr }}, A_{t}^{\text {de }}, A_{t}^{h s t_{0}}, A_{t}^{h s t_{1}}, A_{t}^{h s t_{2}}, A_{t}^{h s t_{3}}\right]
$$

where $A_{t}$ represents the sequence of HT values generated during week $t$.

$\mathrm{X}_{2}[T]$ is a matrix representation of the weekly HT-KDE distribution estimates from baseline till $t=T$ :

$$
\begin{gathered}
X_{2}[T]=\left[\begin{array}{c}
X_{2, A_{1}} \\
X_{2, A_{2}} \\
\vdots \\
X_{2, A_{T}}
\end{array}\right] \\
X_{2, A_{t}}=\left[\rho_{K, A_{t}}, \operatorname{mask}[t], \operatorname{delta}[t]\right]
\end{gathered}
$$

Over the follow-up period participants presented diverse typing behaviours regarding the level of laptop-use and typing activity. In some cases, low typing activity led to gaps (full weeks with no data) in the keystroke data. To standardise the shape of the input sequences we used a "last observation carried forward" imputation method to fill in the missing values, i.e. we select the immediately prior sample to impute the missing value. Two notify the model about the presence of imputed data two additional parameters are incorporated to the input data: mask $[T]$, 
a binary feature to mark the position of the imputed sequence elements ( 1 imputed, 0 original), and delta[T], a temporal reference that indicates the relative position of the current element with regard to the last available populated element in the input sequence n number of weeks (Che et al., 2016).

\section{Multivariate Analysis}

The multivariate model introduced in this chapter uses a Multi-Task Learning (MTL) approach to evaluate participants' response to medication via the analysis of the keystroke data collected from their daily interaction with their laptop at home. In machine learning, MTL describes a learning paradigm in which multiple loss functions associated to a series of related tasks are optimized jointly to improve the model's ability to generalize of the target task, reducing the risk of overfitting (Ruder, 2017). Our model has been designed to focus on two tasks:

1. Classification of Parkinson's disease (PD) versus healthy control typing phenotypes (TASK 1): This branch of the model is based on our previous work, presented in Chapter 5 (Giancardo et al., 2016; Arroyo-Gallego et al., 2018a), where we showed that the typing data from users' daily interaction with their personal devices can be used to derive an early PD phenotype biomarker. The task input, $X_{1}[T]$, uses the same HT based features introduced in Giancardo et al., but instead of using 90 second typing intervals as independent data units, the features are computed on a weekly basis and cumulatively averaged over time from baseline till week $T$. This task sub-model consist of two fully-connected neural network layers with a sigmoid activation function to generate the final sub-score.

2. Detection of positive response to dopaminergic intervention (TASK 2): This model branch focuses on the identification of long-term changes in participants' typing patterns. The longitudinal typing signal is modeled as timesequence of HT probability distributions $\left(X_{2}[T]\right)$ that is used as the input to a recurrent neural network model (RNN). Specifically, we designed a neural network consisting of a series of Long Short-Term Memory units (LSTM) (Hochreiter and Schmidhuber, 1997). LSTMs are a special kind of RNN specifically designed to be sensitive to temporal changes in the input sequences. This allows this model branch to evaluate changes in participants typing patterns over time and identify the temporal patterns affected by the effect of medication.

The outputs from these two tasks are combined in a final shared module that uses the Softmax activation function to provide the predicted probability of belonging to each of the group cases: healthy control, PD non-responders and PD responders. The final score, or $n Q R N N$, is defined by the probability of being classified as PD responder. The joint model was trained in 80 epochs using RMSprop optimization, with learning rate of $\eta=0.001$, and cross-entropy loss functions (Janocha and Czarnecki, 2017) on each of the task modules.

Given the expression that defines the cross-entropy loss in a multiclass classification problem (Deep learning):

$$
L(p, q)=-\sum_{m=1}^{M} p(m) \cdot \log (q(m))
$$


FIGURE 6.3: Feature extraction for At-Home data. This visual represents the data processing designed to define a standard input for the longitudinal at-home typing data. On the first step (A), the keystroke data was grouped by weeks. We used kernel density estimation (KDE) to generate a representation of the distribution of hold times (HT) within that week of data. Additionally, we computed a feature vector as the one described in Chapter 5. For those weeks where not enough typing data was available to compute the analysis, we applied an data imputation method that took the latest available observation to fill the gap. These cases were marked with two additional features: mask, indicating original versus imputed samples, and delta, indicating the timeframe in weeks between the current sample and the data used to fill the gap.

\section{A Feature extraction (week level)}
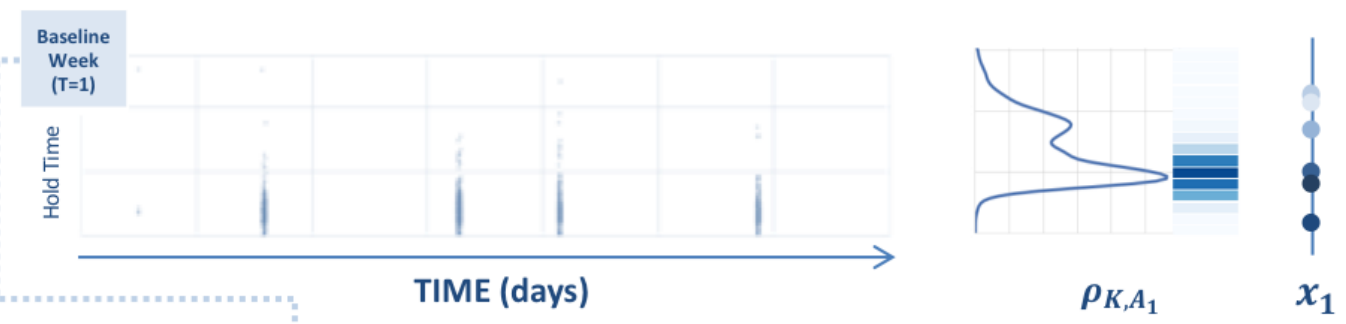

B Feature extraction (longitudinal)




where $M$ is the number of classes, $q$ is the estimated probability distribution and $p$ is true distribution. The loss function for each task in the $n Q R N N$ is defined as follows:

$$
\begin{aligned}
& L_{T A S K 1}=L\left(p_{P D \text { vs CNT }}, q_{P D \text { vs CNT }}\right) \\
& L_{\text {TASK2 }}=L\left(p_{\text {Change vs No-Change }}, q_{\text {Change vs No-Change }}\right) \\
& L_{M E R G E}=L\left(p_{\text {Control vs } P D_{\text {Change }} \text { vs } P D_{\text {No-Change }}} q_{\text {Control vs } P D_{\text {Change }} \text { vs } P D_{\text {No-Change }}}\right)
\end{aligned}
$$

The overall model loss is computed as the weighted sum of the tasks losses:

$$
L_{n Q R N N}=w_{T A S K 1} L_{T A S K 1}+w_{T A S K 2} L_{T A S K 2}+w_{M E R G E} L_{M E R G E}
$$

with $w_{T A S K 1}=w_{T A S K 2}=0.2$ and $w_{M E R G E}=1$.

A detailed representation of the model architecture is presented in Figure 6.4.

To evaluate the model we used a stratified k-fold cross-validation approach (Kohavi, 1995) that provides a good representation of the whole sample class distribution on each of the training folds to reduce the algorithm bias potentially introduced by class imbalance.

\section{Analysis methods}

We evaluated the ability of the proposed metrics to identify participants in the PD group that responded to medication (positive class). To define the negative class we used two approaches, one that aggregated the results from both PD non-responders and healthy controls in a joined no-change category, and another one where we only included PD non-responders for a strict analysis of the metrics performance in the Parkinson's group.

The metrics were evaluated at two different time points over the follow-up period. First, to assess the ability of the proposed methods to predict the long term response to medication, we compared the metrics performance measured at $T=8$, i.e. eight weeks from baseline, to the final response classification as defined by the MCIC for the full six-month follow-up. Second, we evaluated the agreement between our typing-based metrics and the MCIC definition of response at matching time points, i.e. using the scores computed at follow-up completion $(T=24)$.

The results obtained in each analysis scenario were evaluated using the following metrics: receiver operating characteristic (ROC) analysis and the Mann-Whitney $\mathrm{U}$ test to reject the null hypothesis that the typing metrics for PD responders and non-responders (including and excluding controls) come from the same distribution. For the ROC analysis we used a sampling with replacement method to define a distribution of curves from which we computed the average area under the curve (AUC) and its confidence intervals. Each curve is built on an iterative process that monotonically increases the value of the index to define a dynamic threshold. On each iteration, a sensitivity/specificity pair is computed using the current threshold value. This pairs are used to draw the resulting ROC curve. The value of the AUC can be interpreted as the probability of the classifier to rank a randomly chosen positive instance higher than a randomly chosen negative one (Fawcett, 2006). Finally, we included the percent agreement between the classification obtained for the typing metrics and the one defined by the UPDRS-III MCIC definition. The typing metrics cut-offs were estimated using the closest-to- $(0,1)$ on the corresponding 
FIGURE 6.4: nQRNN model architecture. The nQRNN model is designed as a multitask entity. The first task adapts the pre-processing stage from the nQi algorithm to assess the presence or absence of PD motor features in the typing signal. The second task is designed to identify change over time in the longitudinal typing signatures. The results are merged to generate a signal of nQRNNs scores, one per week in the input signal, designed to predict and detect positive response to medication. As for the network architecture, the first task, PD vs CNT, uses fully-connected layers to analyze the feature based data from the at-home typing data. The second task, Change vs NoChange, uses a combination of long-short term memories (LSTM) and fully-connected layers to reduce the information included in the time series of weekly hold time distributions in the at-home data. The outputs from these two tasks are merged using fully-connected layers. The nQRNN model uses the probability of being identified as a PD responder as the final outcome.

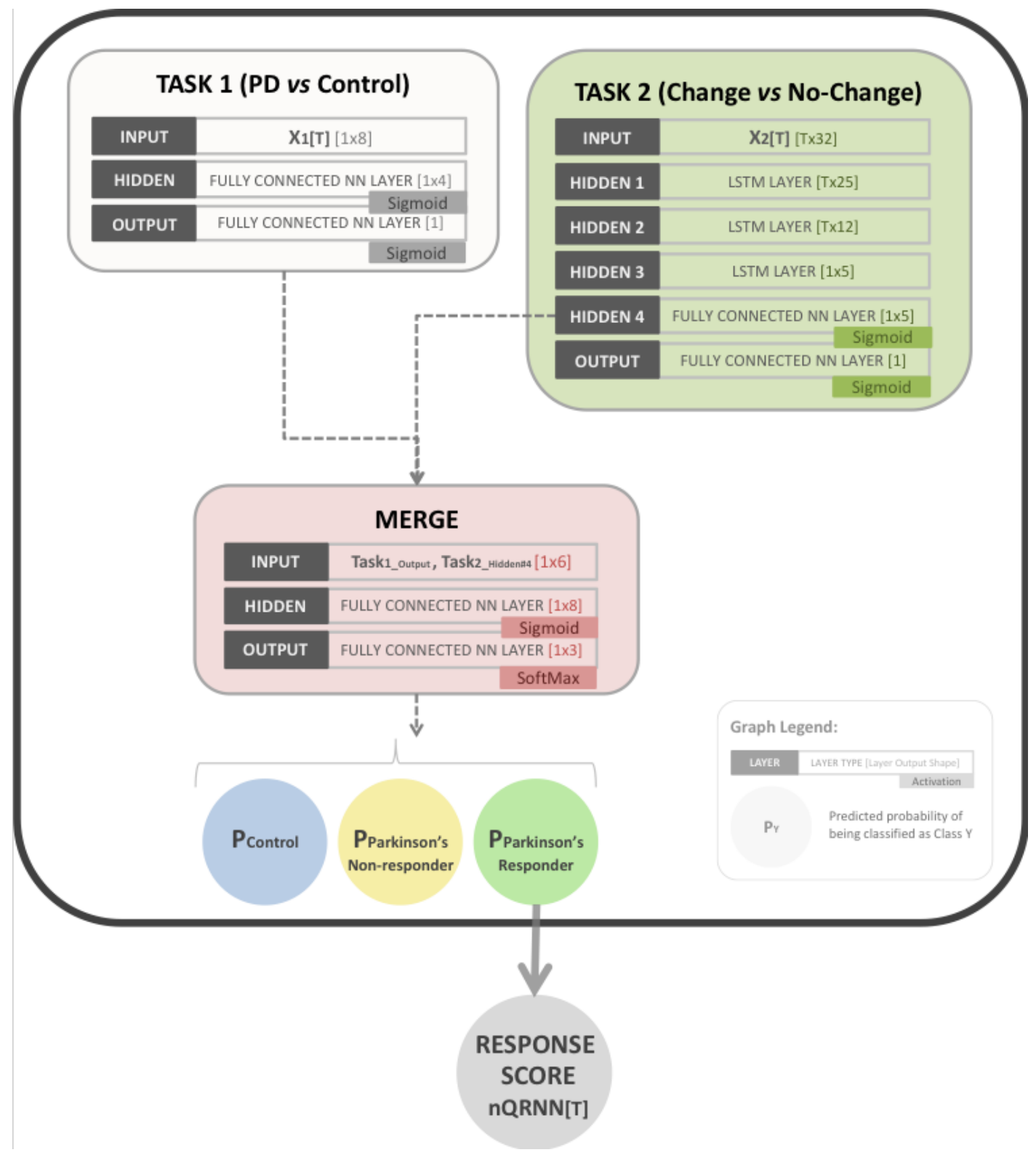


ROC curves, i.e. the use case that maximizes the sensitivity/specificity pair (Perkins and Schisterman, 2006).

\subsection{Results}

Boxplots showing the class separation achieved using the in-clinic distribution distancebased metrics are presented in Figure 6.5. The figure displays the results at weeks 8 and 24 relative to baseline. A similar analysis is presented in Figure 6.6 for the athome nQRNN score. In this case, we also included a longitudinal representation of the model output over the follow-up period. In both cases, in-clinic and at-home, a greater overlap was observed between the scores of controls and PD participants that did not present a significant response to therapy versus PD participants that showed a significant improvement at the completion of the study period. The nQRNN signal becomes stable by week 8 from baseline, keeping a consistent separation between the "No-Change" and PD responders groups for the rest of the follow-up period.

As for classification performance, a comparison of the ROC curves built for each of the proposed metrics is presented in Figure 6.7. The ROC analysis is presented for prediction of final responses assessment at week 8 and agreement with response assessment at week 24 from baseline. Relative to class distribution, two scenarios are included. One that assess the metrics' ability to distinguish PD responders from "No-Change", including PD non-responders and healthy controls in this second category. The nQRNN was able to separate PD responders from the "No-Change" with AUCs of $0.85^{* * *}$ and $0.80^{* *}$ at weeks 8 and 24 respectively. In the in-clinic setting, the convolution-based metric was the one that provided the best separation according to the ROC analysis (AUC of $0.88^{* * *}$ for both weeks 8 and 24). The second one analyzes a strict PD response classification scenario, where the metrics' are evaluated by their ability to separate PD responders versus PD non-responders. In this scenario, the nQRNN was able to separate PD responders from PD non-responders with AUCs of $0.83^{*}$ and 0.74 at weeks 8 and 24 respectively. In the in-clinic setting, the Bhattachayyara-based metric was the one that provided the best separation at week 8 (AUC of $0.86^{*}$ ) and both convolution and correlation-based were the best ones measured at week 24 (AUC of 0.80). For all metrics, classification performance is better when separating responders from the "No-Change" group than the one achieved when classifying response strictly in the PD group. Additionally, all metrics tend to perform better at predicting response at Week 8 from the baseline visit versus the agreement scenario where we compare time coincidence outputs. Table 6.2 summarizes the performance of the proposed metrics for each of the analysis scenarios.

\subsection{Discussion}

The main objective of this study was to determine the feasibility to apply our typingbased methodology to detect changes in response to medication in PD. For this, we designed a naturalistic study where recently diagnosed dopaminergic-agent-free Parkinson's disease participants (PD) and healthy controls (CNT) were studied over the course of six months. We developed and evaluated four different metrics based on typing pattern analysis, from both in-clinic and at-home data, with particular emphasis on measuring changes that reflected a response to drug therapies. Our design 
FIGURE 6.5: In-Clinic results: statistical distance metrics. This subplot represents the separation achieved by the proposed statistical distance metrics proposed for the analysis of the in-clinic hold time distributions. The results are presented for both, the prediction scenario (Week 8) and agreement at the end of the follow-up (Week 24).
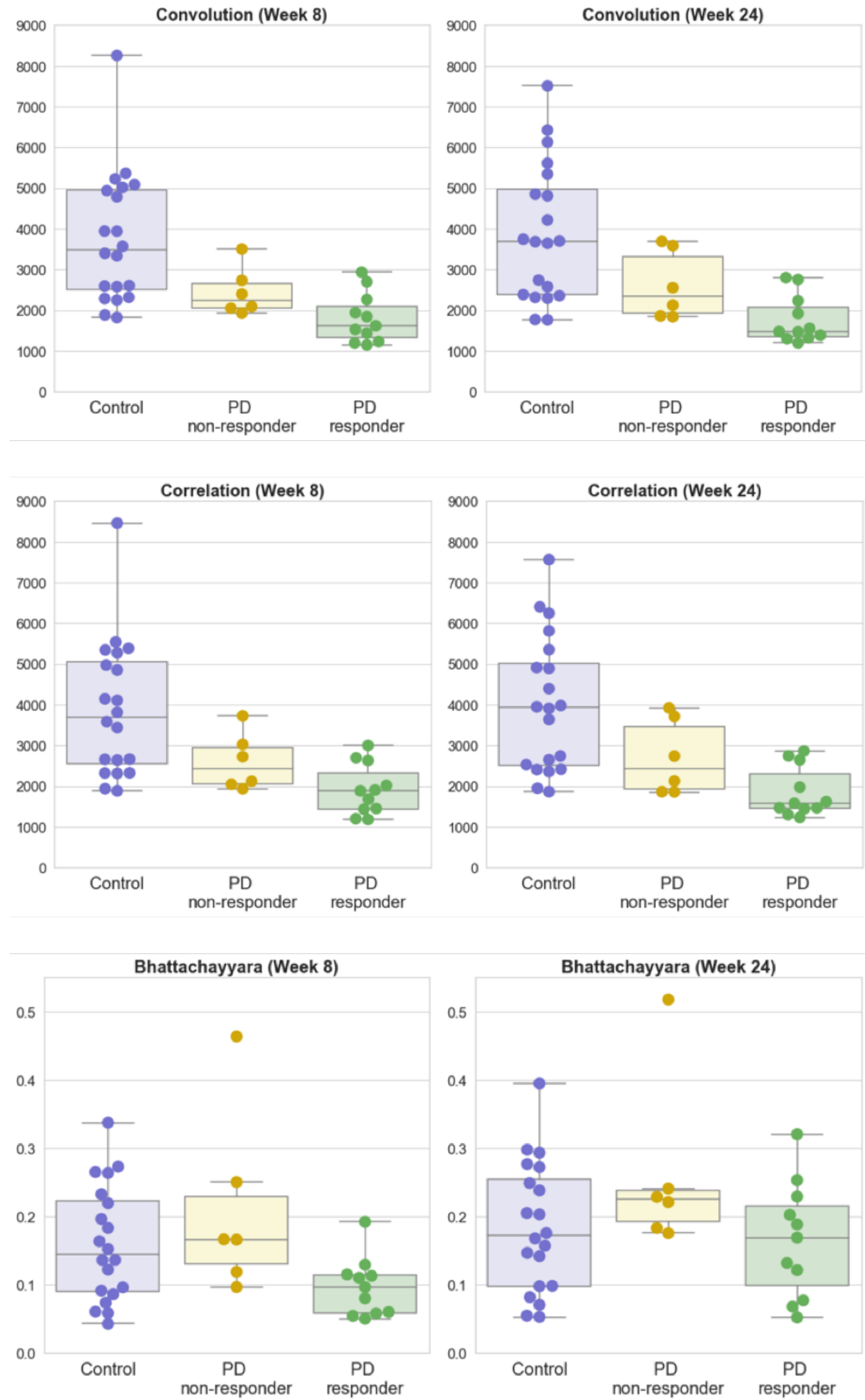
FIGURE 6.6: At-Home results: nQRNN model. Similar to Figure 6.5, this graph presents the results for the nQRNN score both, for prediction and agreement with the standard at the end of the study period. Given the longitudinal nature of the data used in this scenario, a representation of the time series of $n Q R N N$ scores computed over time is shown in Panel B.
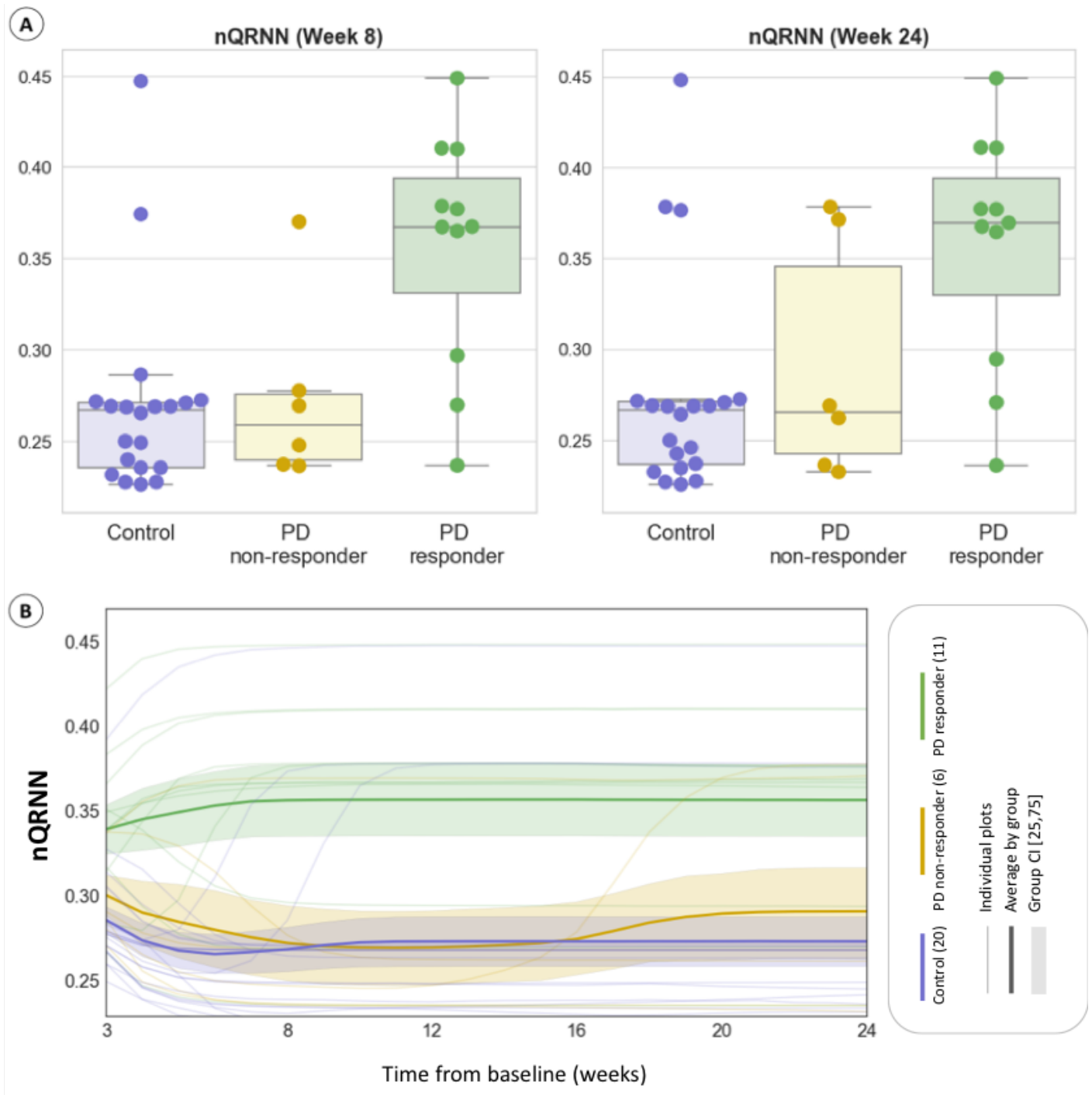
FIGURE 6.7: ROC analysis comparison. Comparison of receiver operating characteristic (ROC) curves showing the classification rate for the typing based metrics, the three statistical distance-based metrics (convolution, correlation and Bhattachayyara) the at-home nQRNN. Statistical significance of the Mann-Whitney $U$ test is estimated to reject the null hypothesis that the two groups, PD responders and nochange/non-responders, come from the same population. Statistical significance noted as: $\mathrm{p}<0.001\left(^{* * *}\right), \mathrm{p}<0.01\left(^{* *}\right)$ and $\mathrm{p}<0.05\left(^{*}\right)$.
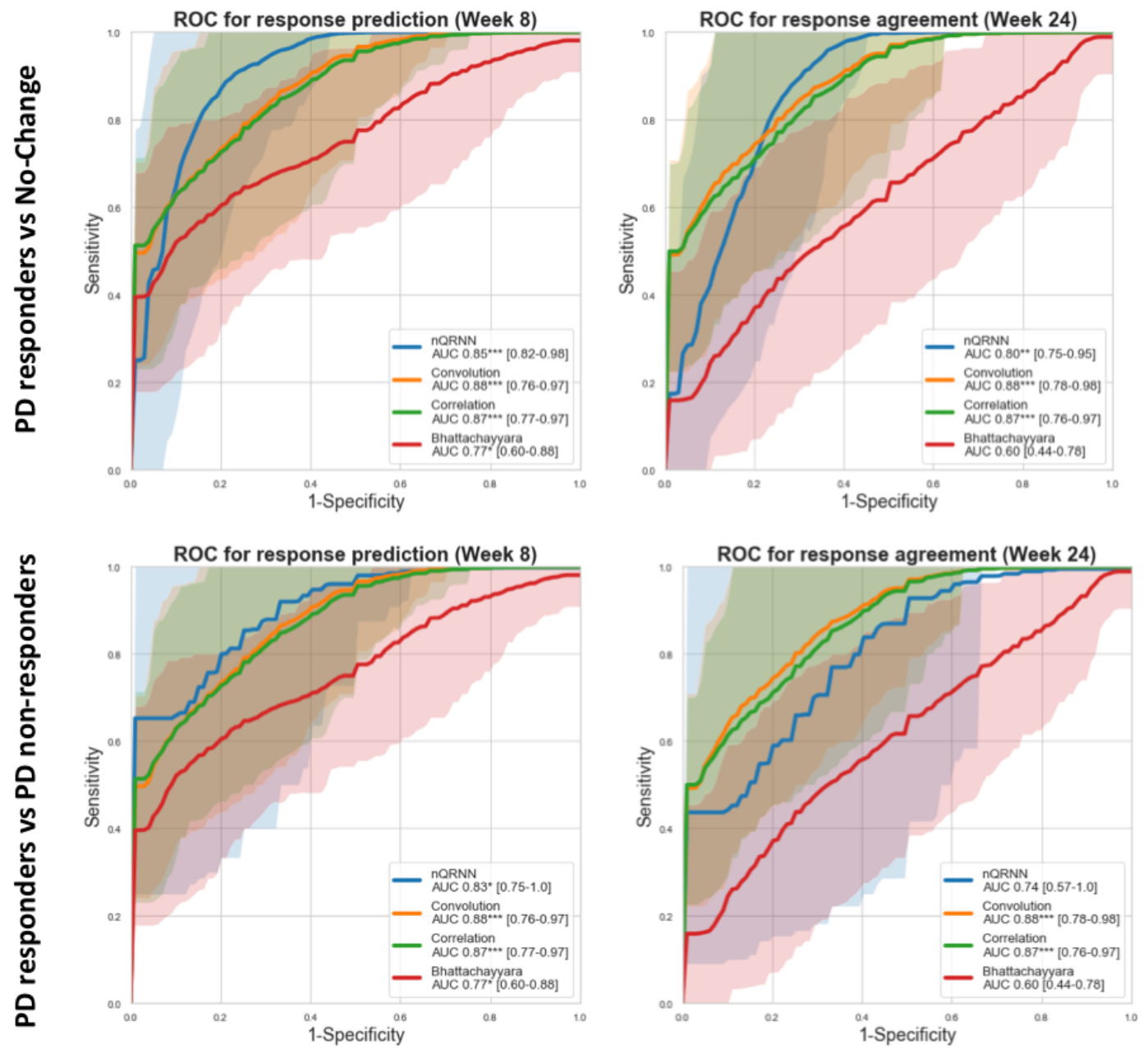
TABLE 6.2: Metrics performance summary. The table represents the performance achieved by the proposed metrics, both for the ones generated in-clinic and at-home, in four different scenarios defined by, inclusion or exclusion of healthy controls in the No-Change group, and time relative to the completion of the study when the scores where evaluated (i.e. prediction vs agreement with response). For each metrics we present the Area Under the ROC curve (AUC), significance defined by the Mann-Whitney U, estimated to reject the null hypothesis that the two groups under study come from the same population, and the percentage agreement achieved by the metric under study.

\begin{tabular}{|c|c|c|c|c|c|c|c|}
\hline & \multicolumn{3}{|c|}{ Prediction (Week 8) } & \multicolumn{3}{|c|}{ Agreement (Week 24) } \\
\hline & & $A U C$ & $p$-value & $\%$ Agreement & $A U C$ & $p$-value & \% Agreement \\
\hline \multirow{4}{*}{$\begin{array}{c}\text { PD responders } \\
\text { vs } \\
\text { No-Change }\end{array}$} & $n Q R N N$ & 0.85 & $* * * \mathrm{p}<0.001$ & $84 \%$ & 0.80 & $* * p<0.01$ & $79 \%$ \\
\hline & Convolution & 0.88 & $* * * \mathrm{p}<0.001$ & $70 \%$ & 0.88 & $* * * \mathrm{p}<0.001$ & $70 \%$ \\
\hline & Correlation & 0.87 & $* * * p<0.001$ & $70 \%$ & 0.87 & $* * * \mathrm{p}<0.001$ & $70 \%$ \\
\hline & Bhattachayyara & 0.77 & $\mathrm{p}<0.05$ & $65 \%$ & 0.60 & $\mathrm{p}=0.34$ & $65 \%$ \\
\hline \multirow{4}{*}{$\begin{array}{c}\text { PD responders } \\
\text { vs } \\
\text { PD non-responders }\end{array}$} & $n Q R N N$ & 0.83 & $* p<0.05$ & $77 \%$ & 0.74 & $p=0.12$ & $71 \%$ \\
\hline & Convolution & 0.80 & $\mathrm{p}=0.05$ & $65 \%$ & 0.80 & $\mathrm{p}=0.05$ & $65 \%$ \\
\hline & Correlation & 0.83 & ${ }^{*} \mathrm{p}<0.05$ & $65 \%$ & 0.80 & $p=0.05$ & $65 \%$ \\
\hline & Bhattachayyara & 0.86 & ${ }^{*} \mathrm{p}<0.05$ & $70 \%$ & 0.73 & $\mathrm{p}=0.15$ & $64 \%$ \\
\hline
\end{tabular}

succeeded in observing a clinically meaningful change similar to what could be expected in a drug trial. We defined the response to medication as a 5-point or greater improvement in the UPDRS-III score.

We successfully designed and deployed a software infrastructure to remotely capture finger interaction variables during controlled and uncontrolled typing sessions in the clinics and at the subjects' homes. This allowed us to collect months' worth of longitudinal variables representing the timing between press and release of keys with precision in the order of milliseconds. These longitudinal data spanned transitions in drug therapies and so were representative of a design that might be used in an interventional drug trial.

The proposed metrics were able to capture significant differences between participants that responded to medication versus participants whose motor state remained stable thorough the follow-up period. In-clinic, statistical distance metrics were all able to predict the outcome of therapy at the end of the follow-up from week 8 with an AUC of 0.88 for the best performing metric. The at-home-based model, $n Q R N N$, was able to discriminate responders from non-responders with an AUC of 0.83 and 0.74 at week 8 and final visit respectively. As expected, non-significant differences were found in the proposed typing metrics between non-responders and healthy controls.

Moreover, the $n Q R N N$ score was able to show significant separation between responders and non-responders from three weeks from the baseline visit, becoming stable at Week 8 from treatment start. These findings suggest that our tool may be sensitive to subtle motor changes, being able to detect people that are going to respond to medication months before the current standard is able to reflect a minimally clinical important change. This could be crucial for example in supporting go/no-go decisions in early intervention trials reducing the cost of developing new compounds and also potentially being helpful to adjust treatments in clinical practice.

Although these results are promising, our study has some limitations that should be considered in future efforts to validate this method. First, even though we used a cross-validation approach that allowed us to test the generalizability of our model 
in a limited dataset, our cohort does not provide a complete representation of all nuances of PD progression. More complex models, such as deep networks, are likely to capture more complex typing patterns and provide a better representation of response. However, due to the limited size of our dataset, it was not possible to test this hypothesis. Additionally, a more representative cohort should include negative response or worsening trajectories in the progression of the disease. Due to limitations of the study dataset, only stable and positive response to medication were characterized by the proposed metrics. Being able to differentiate improved, stable and worsen motor performance and more extensive validation on independent cohorts would be critical to ensure the translatability of the proposed method to everyday practice and clinical trials.

In conclusion, we report in this pilot study the design, implementation and use of a a novel technological approach to monitor motor features of PD and drug response remotely and ecologically in an accurate way, reflecting the underlying effects of basal ganglia neurodegeneration on a habitual task, such as typing. Our findings support the validity of the proposed methodology to generate typing-derived measures as complementary outcomes in interventional drug trials, bringing us a step closer to using artificial intelligence to model the natural interaction with consumer electronics and measure relevant aspects of Parkinson's disease. 


\section{Chapter 7}

\section{Summary and Conclusions}

This $\mathrm{PhD}$ work focuses in the exploration of a novel solution that addresses an unmet need in the management of Parkinson's Disease (PD), the lack of objective invivo biomarkers for the diagnosis and monitoring of the progression of the disease.

PD is one of the most prevalent movement disorders and the second most common neurodegenerative disease in the western world. It is a highly debilitating disease, characterized by the presence of motor degradation resulting from the loss of nigrostriatal dopaminergic neurons in the central nervous system. Non-motor signs, such as gastrointestinal, olfactory, or autonomic symptoms, mood alteration or impulse control disorders, cognitive alterations and sleep disorders, are also hallmarks of this disease.

During the prodromal and early stages of the disease, subtle motor impairment can precede the clinical diagnosis of PD that can often go unnoticed for several years. Current clinical practices fail to provide a prompt diagnosis, which can lead to aggravation of the clinical course of the disease. After diagnosis, physicians have limited access to consistent and objective data to optimize treatment decisions. Infrequent clinical examinations can only provide a snapshot of patient's status, leaving large gaps of time where physicians are blind to changes in the disease. Thus, finding high-quality measurements to assess the symptoms of the disease is among the top priorities in the research and clinical agendas for PD.

This work presents a novel assessment tool that aims to continuously and objectively characterize psychomotor performance and fine motor abnormalities in PD to support more efficient diagnosis and management of existing therapies.

Based on the analysis of the timing patterns built from fingers interactions with mechanical and touchscreen keyboards, we developed a series of algorithms that introduce a collection of digital markers to generate computational outcomes measurements for the management of PD.

\subsection{Hypothesis and Objectives Revisited}

The main hypothesis motivating this work stated that:

"Valuable information about individuals' psychomotor health can be derived from the timing data patterns extracted from routine finger-keyboard interactions in computers and smartphones."

Based on this idea, the goal of this thesis was to evaluate the feasibility of creating new methods for the assessment of motor deterioration in PD through the analysis of user-device interactions. We broke down this goal into three primary objectives that we empirically tested generating the following results: 


\section{Objective 1 - At-home validation of mechanical keyboard patterns.}

We validated our approach to derive characteristic patterns of early Parkinson's from the analysis of keystroke data collected from patients' routine use of computers at home. We implemented the data-collection platform and software to enable access and storage of the typing data generated by users in a real world setting. We recruited a total of 60 participants; of these participants 52 (25 Parkinson's patients and 27 healthy controls) provided enough data to complete the analysis. Finally, to evaluate whether our in-clinic-built algorithm could be used in an uncontrolled at-home setting, we compared its performance on the data collected during the controlled typing task in the clinic and the results of our method using the data passively collected at home.

Despite the randomness and sparsity introduced by the uncontrolled setting, our algorithm performed nearly as well in the at-home data (AUC of 0.76 and sensitivity/specificity of $0.73 / 0.69$ ) as it did when used to evaluate the inclinic data (AUC 0.83 and sensitivity/specificity of $0.77 / 0.72$ ). Moreover, the keystroke metrics presented a strong correlation between the two typing settings, which suggests a minimal influence of the in-clinic typing task in users' normal typing.

The finding that an algorithm trained on data from an in-clinic setting has comparable performance with that tested on data collected through naturalistic athome computer use reinforces the hypothesis that subtle differences in motor function can be detected from typing behavior.

2. Objective 2 - Implementation of smartphone-based keyboard pattern for PD.

Typing kinematics vary widely between mechanical and touchscreen keyboards. We developed and validated a new methodology to quantify PD motor impairment through the analysis of the keystroke signals collected via smartphone devices.

We proposed a set of touchscreen typing features based on a covariance, skewness, and kurtosis analysis of the timing information of the data to capture PD motor signs. We tested these features, both independently and in a multivariate framework, in a population of $21 \mathrm{PD}$ and 23 control subjects, achieving a sensitivity/specificity of $0.81 / 0.81$ for the best performing feature and $0.73 / 0.84$ for the best multivariate method. The results of the alternating fingertapping, an established motor test, measured in our cohort are $0.75 / 0.78$. This paper contributes to the development of a home-based, high-compliance, and high-frequency PD motor test by analysis of routine typing on touchscreens.

3. Objective 3 - Implementation and design of longitudinal models to measure medication response.

The ability to assess response to drug treatments in between clinician visits can provide early confirmation of the optimal dosing regimen or provide prompt evidence to interrupt ineffective treatments. We developed and evaluated new models designed to predict patients' response to dopaminergic therapy through the analysis of their typing routines in-clinic and at-home.

The proposed metrics were able to identify patients responding to levodopa months earlier than the current standard reached a minimally clinical important change. Our approach was tested in two different settings: 1) in a controlled typing environment (in-clinic), where the proposed metrics were able 
to identify patients with a positive response to therapy with an AUC of 0.88 and, 2) in a real world setting (at-home), where a model based on recurrent neural layers was able to discriminate responders from non-responders with an AUC of 0.83 by the eighth week in a six-month follow-up study.

\subsection{Conclusions}

This work is intended to represent a step toward an objective, user-convenient, and quasi-continuous detection and monitoring tool for PD. Based on the analysis of the routine typing signal, the proposed approach introduces a transparent way to characterize the psychomotor phenotype of PD.

Capturing keystroke dynamics patterns in today's computers and smartphones, this approach is able to characterize fingers motion at a millisecond level. This highly sensitive representations of routine fine motor tasks has the potential to identify signs of motor decline that may be imperceptible to the human eye. This could provide a more granular and objective representation of the disease to support physicians diagnosis and treatment decisions.

As it does not require a proprietary device, the solution presented in this thesis is inexpensive and widely deployable. As it runs in the background of users' personal devices, this tool could be easily accessible by the general population, and applied in the clinical community for screening, detection and monitoring of psychomotor performance in a real world setting.

Compared to traditional symptom tracking techniques, this solution increases patient compliance, as it runs passively in the background of a user's device and collects keystroke data from patients' natural interactions with their personal devices, without requiring patients to complete specific structured tasks.

\subsection{Impact and Future Work}

Characterizing impaired psychomotor performance caused by neurodegeneration through the analysis of natural keystroke patterns presents an opportunity to tackle some difficult challenges in the diagnosis and management of chronic progressive diseases like PD. The objective, quasi-continuous, unobtrusive, and remote nature of this approach allows for unique contributions to disease management.

Degradation of fine motor control is one of the early manifestations of PD and it aggravates as the disease progresses. Being able to finely quantify this motor feature from the analysis of users' daily interaction with their personal devices has the potential to improve the diagnosis of disease, monitoring of disease progression, and the effectiveness of tracking therapeutic interventions. The proposed approach could set the path to the introduction of a series of computational biomarkers that define a digital phenotype of PD able to identify subtle features and changes in the disease, supporting the development of neuroprotective or neuromodifying therapies by enabling early detection during the prodromal phase of the disease.

This research shows the technical feasibility of capturing clinically relevant characteristics of early PD by analyzing patients' interactions with computer and touchscreen keyboards. Although our work represent just initial validation for the proposed methodology, these results show promise in the development of an assessment tool that could potentially accompany clinical decisions thorough the whole disease cycle. 
In patients at risk or suspected of having PD, the proposed method could help detecting subtle degradation of fine motor performance and assist in physicians to improve the diagnostic accuracy of PD related symptoms. Fine motor degradation precedes onset of PD neurodegeneration, which is often missed by current clinical standards. The proposed tool could provide a more sensitive way to capture these early signs of PD and enable earlier detection, pushing clinical intervention closer to the disease onset.

In patients diagnosed with $\mathrm{PD}$, the ability to assess response to drug treatments in between clinician visits can not only provide early confirmation of the optimal dosing regimen, but also shorten the time patients receive ineffective treatments. In the absence of neuroprotective drugs, optimizing symptomatic relief provided by currently available treatments constitutes one of the main priorities as it could maximize daily function and patients' quality of life.

In later stages of the disease, and particularly in patients who become refractory to dopaminergic therapies and undertake surgical intervention with deep brain stimulation (DBS), the ability to objectively measure the impact of electroestimulation in fine motor performance could help illustrate the therapeutic benefit of these procedures and provide a quantitative endpoint to guide system calibration in order to optimize and tailor stimulation parameters to patients' needs.

In the world of drug discovery, where the current standards for the development of new therapies, randomized clinical trials, have limitations to provide a complete representation of the actual outcomes of new therapies, a real world data approach, like the one proposed in this work, could help provide broader scientific evidence and a better understanding of new drugs' effectiveness and safety. Real world evidence can enrich clinical trial design, increasing power by shortening follow up time and sample size. Specifically in diseases like PD, where early signs of neurodegeneration can go unnoticed for years, a tool like this could help enable early screening of large populations and more specific profiling of trial participants.

In this thesis we have presented a series of algorithms based on the analysis of single keystroke (HT) or two consecutive keystroke-metric analysis (FT). In future work, more complex patterns for keystroke representation, such us multi-keystroke or touchscreen gesture analysis (e.g. drag-swipe or pinch-spread) could be introduced to provide a richer representation of signal inputs, enabling the analysis of more complex finger-device dynamics.

Finally, our view is that this approach is not limited to PD. Typing is a complex process that involves coordination of multiple functional pathways, including fine motor control, cognitive processes and sensory-motor feedback. Being able to identify the specific signature of different forms of neurodegeneration from typing could help better delineate overlapping and cardinal sings of different clinical conditions that could also benefit from this technology. 


\section{Bibliography}

Aarsland, Dag et al. (2004). "The Rate of Cognitive Decline in Parkinson Disease". In: Archives of Neurology 61.12, pp. 1906-1911. ISSN: 0003-9942. DOI: 10.1001 / archneur . 61 . 12 . 1906. URL: http: / / archneur . jamanetwork . com/article . aspx?doi=10.1001/archneur.61.12.1906.

Abós, Alexandra et al. (2017). "Discriminating cognitive status in Parkinson's disease through functional connectomics and machine learning". In: Scientific Reports 7.1, p. 45347. ISSN: 2045-2322. DOI: 10.1038/srep45347. URL: http://www . nature.com/articles/srep45347.

Achey, Meredith et al. (2014). "The past, present, and future of telemedicine for Parkinson's disease". In: Movement Disorders 29.7, pp. 871-883. ISSN: 08853185. DOI: 10 . 1002 /mds . 25903. URL: http : / / www . ncbi . nlm . nih . gov/pubmed / 24838316http://doi.wiley.com/10.1002/mds. 25903.

Adams, Christopher P. and Van V. Brantner (2006). "Estimating The Cost Of New Drug Development: Is It Really $\$ 802$ Million?" In: Health Affairs 25.2, pp. 420428. ISSN: 0278-2715. DOI: 10 . 1377 / hlthaff . 25 . 2 . 420. URL: http : / / www . healthaffairs.org/doi/10.1377/hlthaff.25.2.420.

Adams, Warwick R (2017). “High-accuracy detection of early Parkinson's Disease using multiple characteristics of finger movement while typing". In: PLOS ONE 12.11. Ed. by Holger Fröhlich, e0188226. ISSN: 1932-6203. DOI: $10.1371 /$ journal . pone.0188226. URL: https://dx.plos.org/10.1371/journal . pone.0188226.

Ahlskog, J. E. and R. J. Uitti (2010). "Rasagiline, Parkinson neuroprotection, and delayed-start trials: Still no satisfaction?" In: Neurology 74.14, pp. 1143-1148. ISSN: 0028-3878. DOI: 10 . 1212/WNL . Ob013e3181d7d8e2. URL: http ://www . ncbi . nlm . nih.gov/pubmed/20368634http://www . pubmedcentral.nih.gov/articlerender. fcgi ?artid=PMC2865777http: //www . neurology . org/cgi/doi/10.1212/WNL . Ob013e3181d7d8e2.

Ahmad, Nasir, Andrea Szymkowiak, and Paul A. Campbell (2013). "Keystroke dynamics in the pre-touchscreen era". In: Frontiers in Human Neuroscience 7, p. 835. ISSN: 1662-5161. DOI: 10 .3389/fnhum. 2013.00835. URL: http://www .ncbi .nlm. nih.gov/pubmed/24391568http://www . pubmedcentral.nih.gov/articlerender . fcgi?artid=PMC3867681http://journal. frontiersin.org/article/10.3389/ fnhum. 2013.00835/abstract.

Al-Obaidi, Noor Mahmood and Mudhafar M. Al-Jarrah (2016). “Statistical Keystroke Dynamics System on Mobile Devices for Experimental Data Collection and User Authentication". In: 2016 9th International Conference on Developments in eSystems Engineering (DeSE). IEEE, pp. 123-129. ISBN: 978-1-5090-5487-9. DOI: 10.1109/ DeSE. 2016.21. URL: http://ieeexplore . ieee.org/document/7930635/.

Ali, Md Liakat et al. (2017). "Keystroke Biometric Systems for User Authentication". In: Journal of Signal Processing Systems 86.2-3, pp. 175-190. ISSN: 1939-8018. DOI: 10. 1007 / s11265-016-1114-9. URL: http : / / link . springer . com/10 . 1007 / s11265-016-1114-9.

Araujo, L.C.F. et al. (2005). "User authentication through typing biometrics features". In: IEEE Transactions on Signal Processing 53.2, pp. 851-855. ISSN: 1053-587X. DOI: 
10. 1109/TSP . 2004 . 839903. URL: http : / / ieeexplore . ieee .org/document / $1381785 /$.

Arora, S. et al. (2015). "Detecting and monitoring the symptoms of Parkinson's disease using smartphones: A pilot study".

Arroyo-Gallego, T et al. (2018a). "Detecting Motor Impairment in Early Parkinson's Disease via Natural Typing Interaction with Keyboards: Validation of the neuroQWERTY Approach in an Uncontrolled At-Home Setting". In: Journal of medical Internet research (in press), pp. 1-2. ISSN: 1438-8871. DOI: 10.2196/jmir. 9462.

Arroyo-Gallego, T. et al. (2018b). “Detecting Motor Impairment in Early Parkinson's Disease via Natural Typing Interaction With Keyboards: Validation of the neuroQWERTY Approach in an Uncontrolled At-Home Setting". In: Journal of Medical Internet Research 20.3. ISSN: 14388871. DOI: 10.2196/jmir. 9462.

Arroyo-Gallego, Teresa et al. (2017). “Detection of Motor Impairment in Parkinson's Disease via Mobile Touchscreen Typing". In: IEEE Transactions on Biomedical Engineering, pp. 1-1. DOI: 10.1109/TBME. 2017.2664802. URL: http://ieeexplore. ieee.org/document/7859354/.

Artusi, Carlo Alberto et al. (2018). "Integration of technology-based outcome measures in clinical trials of Parkinson and other neurodegenerative diseases". In: Parkinsonism \& Related Disorders 46, S53-S56. ISSN: 13538020. DOI: $10.1016 / \mathrm{j}$. parkreldis . 2017 . 07 . 022. URL: http : / /www . ncbi . nlm . nih . gov/pubmed / 28760593https://linkinghub.elsevier.com/retrieve/pii/S1353802017302699.

Asakawa, Deanna S., Jack T. Dennerlein, and Devin L. Jindrich (2017). “Index finger and thumb kinematics and performance measurements for common touchscreen gestures". In: Applied Ergonomics 58, pp. 176-181. ISSN: 0003-6870. DOI: 10.1016/ J . APERGO . 2016 . 06 . 004. URL: https : / / www . sciencedirect . com/science / article/pii/S0003687016301156?via\%3Dihub.

Asakawa, Deanna S. et al. (2017). "Fingertip forces and completion time for index finger and thumb touchscreen gestures". In: Journal of Electromyography and Kinesiology 34, pp. 6-13. ISSN: 10506411. DOI: 10 .1016/ j · jelekin.2017. 02 . 007. URL: http://www .ncbi .nlm.nih.gov/pubmed/28314216https://linkinghub . elsevier.com/retrieve/pii/S1050641116301225.

Asgari, M and I Shafran (2010). "Predicting severity of Parkinson's disease from speech". In: 2010 Annual International Conference of the IEEE Engineering in Medicine and Biology. IEEE, pp. 5201-5204. ISBN: 978-1-4244-4123-5. DOI: 10 .1109/IEMBS . 2010.5626104. URL: http://ieeexplore. ieee.org/document/5626104/.

Bandini, Andrea et al. (2017). "Analysis of facial expressions in parkinson's disease through video-based automatic methods". In: Journal of Neuroscience Methods 281, pp. 7-20. ISSN: 0165-0270. DOI: 10 . 1016/ J . JNEUMETH. 2017 . 02 .006. URL: https : //www. sciencedirect.com/science/article/pii/S0165027017300481?via\% 3Dihub.

Banerjee, Salil Partha and Damon Woodard (2012). "Biometric Authentication and Identification Using Keystroke Dynamics: A Survey". In: Journal of Pattern Recognition Research 7.1, pp. 116-139. ISSN: 1558884X. DOI: 10 . 13176/11 . 427. URL: http://www.jprr.org/index.php/jprr/article/view/427/167.

Bayestehtashk, Alireza et al. (2015). "Fully automated assessment of the severity of Parkinson's disease from speech". In: Computer Speech E Language 29.1, pp. 172185. ISSN: 08852308. DOI: 10 . 1016/ j . csl . 2013 . 12 . 001. URL: http : / / www . ncbi .nlm . nih . gov/pubmed/25382935http: / /www . pubmedcentral . nih . gov/ articlerender .fcgi?artid=PMC4222054https : / / linkinghub . elsevier . com/ retrieve/pii/S0885230813001149. 
Beach, Thomas G (2017). "A Review of Biomarkers for Neurodegenerative Disease: Will They Swing Us Across the Valley?" In: Neurology and therapy 6.Suppl 1, pp. 513. ISSN: 2193-8253. DOI: 10 . 1007 / s40120 - 017 - 0072 - x. URL: http : / / www . ncbi.nlm.nih.gov/pubmed/28733961http: //www . pubmedcentral . nih.gov/ articlerender. fcgi?artid=PMC5520818.

Begg, Rezaul. and Marimuthu. Palaniswami (2006). Computational intelligence for movement sciences : neural networks and other emerging techniques. Idea Group Pub, p. 396. ISBN: 9781591408369 . URL: https ://books . google .es/books?id=Cc2fPmV5BHgC\& $\mathrm{pg}=\mathrm{PA} 122 \& \mathrm{lpg}=\mathrm{PA} 122 \& \mathrm{dq}=\% 22 \mathrm{Gait}++$ is $++\mathrm{a}++$ particular ++ semi - automatic + +motor++task++which++isspecifically+sensitive+to+0N $\% \mathrm{E} 2 \% 80 \% 930 \mathrm{FF}+$ changes+of+Parkinsonian+state. \&source=bl\&ots=cknyaHflF3\&sig=lwNn2sMVj_ Xs0j4n5UhPldsH98s\&hl=en\&sa=X\&ved=2ahUKEwiNlb2p2I j f AhXDfMAKHQLuAi4Q6AEwAHoECAkQAQ\# $\mathrm{v}=$ onepage \& $\mathrm{q}=\% 22$ Gait $\% 20 \% 20$ is $\% 20 \% 20$ a $\% 20 \% 20$ particular $\% 20 \% 20$ semi automatic $\% 20 \% 20$ motor $\% 20 \% 20$ task $\% 20 \% 20$ which $\% 20 \% 20$ isspecifically $\%$ 20 sensitive $\% 20$ to $\%$ 200N \% E2 \% $80 \% 930 \mathrm{FF} \% 20$ changes $\% 20$ of $\% 20$ Parkinsonian $\%$ 20state. $\& \mathrm{f}=\mathrm{fal}$ se.

BehavioSec: Continuous Authentication Through Behavioral Biometrics. URL: https : // www . behaviosec. com/.

Bernad-Elazari, Hagar et al. (2016). "Objective characterization of daily living transitions in patients with Parkinson's disease using a single body-fixed sensor". In: Journal of Neurology 263.8, pp. 1544-1551. ISSN: 0340-5354. DOI: 10.1007/s00415016-8164-6. URL: http : / / www . ncbi . nlm . nih . gov/ pubmed/27216626http : //link. springer.com/10.1007/s00415-016-8164-6.

Betts, J. Gordon et al. Anatomy Eamp; Physiology, p. 1335. ISBN: 1938168135. URL: https : / / books . google.com/books/about/Anatomy_Physiology . html ? id= dvVgngEACAAJ.

Biase, Lazzaro di et al. (2017). "Tremor stability index: a new tool for differential diagnosis in tremor syndromes". In: Brain 140.7, pp. 1977-1986. ISSN: 0006-8950. DOI: 10 . 1093/brain/awx104. URL: http: //www.ncbi .nlm.nih.gov/pubmed/ 28459950http : / /ww . pubmedcentral . nih.gov/articlerender.fcgi ?artid= PMC5493195https://academic.oup.com/brain/article/140/7/1977/3782569.

Biase, Lazzaro di et al. (2018). “Quantitative Analysis of Bradykinesia and Rigidity in Parkinson's Disease." In: Frontiers in neurology 9, p. 121. ISSN: 1664-2295. DOI: 10.3389/fneur . 2018.00121. URL: http://www.ncbi.nlm.nih.gov/pubmed/ 29568281http://www . pubmedcentral . nih.gov/articlerender.fcgi?artid= PMC5853013.

Bishop, Christopher M. (2006). Pattern recognition and machine learning. Springer, p. 738. ISBN: 9780387310732.

Bland, J M and D G Altman (1986). "Statistical methods for assessing agreement between two methods of clinical measurement." In: Lancet (London, England) 1.8476, pp. 307-10. ISSN: 0140-6736. URL: http : / / www . ncbi . nlm . nih . gov / pubmed / 2868172.

Bleha, S., C. Slivinsky, and B. Hussien (1990). “Computer-access security systems using keystroke dynamics". In: IEEE Transactions on Pattern Analysis and Machine Intelligence 12.12, pp. 1217-1222. ISSN: 01628828. DOI: 10 . 1109/34 . 62613. URL: http://ieeexplore.ieee.org/document/62613/.

Bleha, S.A. and M.S. Obaidat (1991). "Dimensionality reduction and feature extraction applications in identifying computer users". In: IEEE Transactions on Systems, Man, and Cybernetics 21.2, pp. 452-456. ISSN: 00189472. DOI: 10.1109/21.87093. URL: http://ieeexplore. ieee.org/document/87093/. 
Block, G et al. (1997). "Comparison of immediate-release and controlled release carbidopa/levodopa in Parkinson's disease. A multicenter 5-year study. The CR First Study Group." In: European neurology 37.1, pp. 23-7. ISSN: 0014-3022. DOI: 10.1159/000117399. URL: http://www.ncbi.nlm.nih.gov/pubmed/9018028.

Bloem, Bastiaan R. et al. (2016). "Measurement instruments to assess posture, gait, and balance in Parkinson's disease: Critique and recommendations". In: Movement Disorders 31.9, pp. 1342-1355. ISSN: 08853185. DOI: 10 . 1002 /mds . 26572. URL: http://doi.wiley.com/10.1002/mds. 26572.

Bot, Brian M. et al. (2016a). "The mPower study, Parkinson disease mobile data collected using ResearchKit". In: Scientific Data 3, p. 160011. ISSN: 2052-4463. DOI: 10 . 1038 / sdata . 2016 . 11. URL: http : / / www . nature . com / articles / sdata201611.

Bot, Brian M et al. (2016b). "The mPower study, Parkinson's disease mobile data collected using ResearchKit". In: Nature Scientific Data ePubished, pp. 1-9. ISSN: 2052-4463. DOI: 10.1038/sdata. 2016.11.

Bozyczko-Coyne, D and M Williams (2007). “Neurodegeneration”. In: Comprehensive Medicinal Chemistry II. Elsevier, pp. 193-228. ISBN: 9780080450445. DOI: 10. 1016/ B0-08-045044-X/00168-1. URL: http://linkinghub. elsevier.com/retrieve/ $\mathrm{pii} / \mathrm{B} 008045044 \mathrm{X} 001681$.

Braak, Heiko et al. (2003). "Staging of brain pathology related to sporadic Parkinson's disease". In: Neurobiology of Aging 24.2, pp. 197-211. ISSN: 0197-4580. DOI: 10 . 1016/S0197-4580 (02) 00065 - 9. URL: https : / /www . sciencedirect . com/ science/article/pii/S0197458002000659.

Breit, S. et al. (2008). "Long-term EMG recordings differentiate between parkinsonian and essential tremor". In: Journal of Neurology 255.1, pp. 103-111. ISSN: 03405354. DOI: $10.1007 / \mathrm{s} 00415-008-0712-2$. URL: http://www.ncbi.nlm.nih.gov/ pubmed/18204805http://link. springer.com/10.1007/s00415-008-0712-2.

Brodie, Matthew A. et al. (2014). "Gait as a biomarker? Accelerometers reveal that reduced movement quality while walking is associated with Parkinson's disease, ageing and fall risk". In: 2014 36th Annual International Conference of the IEEE Engineering in Medicine and Biology Society. Vol. 2014. IEEE, pp. 5968-5971. ISBN: 9781-4244-7929-0. DOI: 10 .1109/EMBC . 2014 .6944988. URL: http://www . ncbi .nlm . nih.gov/pubmed/25571356http://ieeexplore.ieee.org/document/6944988/.

Brooks, David J (2008). “Optimizing levodopa therapy for Parkinson's disease with levodopa/carbidopa/entacapone: implications from a clinical and patient perspective." In: Neuropsychiatric disease and treatment 4.1, pp. 39-47.

Brunner, Dani, Fuat Balc1, and Elliot A Ludvig (2012). "Comparative psychology and the grand challenge of drug discovery in psychiatry and neurodegeneration". In: Behavioural Processes 89, pp. 187-195. DOI: 10 . 1016/ j . beproc . 2011. 10.011. URL: https://s3. amazonaws . com/academia.edu.documents/44227508/ Comparative_psychology_and_the_grand_cha20160330-18629-il8iis . pdf? AWSAccessKeyId = AKIAIWOWYYGZ2Y53UL3A \& Expires $=1525030864 \&$ Signature $=$ frFFOLwBXgVCmb9\%2Fxq1N\%2FGGRIRY\%3D\&response-content-disposition=inl.

Bryan, William Lowe and Noble Harter (1897). "Studies in the physiology and psychology of the telegraphic language." In: Psychological Review 4.1, pp. 27-53. ISSN: 0033-295X. DOI: 10.1037/h0073806. URL: http://content . apa.org/journals / rev/4/1/27.

Cabestany, Joan et al. (2013). "REMPARK: When AI and technology meet Parkinson Disease assessment". In: undefined. URL: https : //www . semanticscholar .org/ paper / REMPARK\%3A - When - AI - and - technology - meet - Parkinson- Cabestany P\%C3\%A9rez-L\%C3\%B3pez/2804a982f6357e1df19a8120cbe6d7158c8cdb50. 
Campenhausen, Sonja von et al. (2011). “Costs of illness and care in Parkinson's Disease: An evaluation in six countries". In: European Neuropsychopharmacology 21.2, pp. 180-191. ISSN: 0924977X. DOI: 10.1016/j . euroneuro . 2010 . 08.002. URL: http://www . ncbi.nlm.nih.gov/pubmed/20888737http://linkinghub . elsevier.com/retrieve/pii/S0924977X10001707.

Can, Yekta Said and Fatih Alagoz (2014). "User identification using Keystroke Dynamics". In: 2014 22nd Signal Processing and Communications Applications Conference (SIU). IEEE, pp. 1083-1085. ISBN: 978-1-4799-4874-1. DOI: 10 . 1109 / SIU . 2014.6830421. URL: http://ieeexplore.ieee.org/document/6830421/.

Cancela, J et al. (2010). "A comprehensive motor symptom monitoring and management system: The bradykinesia case". In: 2010 Annual International Conference of the IEEE Engineering in Medicine and Biology. Vol. 2010. IEEE, pp. 10081011. ISBN: 978-1-4244-4123-5. DOI: 10 . 1109/IEMBS . 2010 . 5627775. URL: http: //www.ncbi.nlm.nih. gov/pubmed/21096992http : / ieeexplore . ieee . org/ document/5627775/.

Cancela, J. et al. (2011). "Gait assessment in Parkinson's disease patients through a network of wearable accelerometers in unsupervised environments". In: 2011 Annual International Conference of the IEEE Engineering in Medicine and Biology Society. IEEE, pp. 2233-2236. ISBN: 978-1-4577-1589-1. DOI: 10 . 1109/IEMBS . 2011. 6090423. URL: http://ieeexplore. ieee.org/document/6090423/.

Chang, Chih-Chung and Chih-Jen Lin. "LIBSVM: A Library for Support Vector Machines". In: URL: https://www.csie.ntu.edu.tw/ cjlin/papers/libsvm.pdf.

Che, Zhengping et al. (2016). "Recurrent Neural Networks for Multivariate Time Series with Missing Values". In: URL: http://arxiv.org/abs/1606.01865.

Chen, Pei-Hao et al. (2013). "Gait Disorders in Parkinson's Disease: Assessment and Management". In: International Journal of Gerontology 7.4, pp. 189-193. ISSN: 18739598. DOI: 10 .1016/J . I JGE . 2013.03.005. URL: https : / /www . sciencedirect. com/science/article/pii/S1873959813000410.

Chen, Tuan-Zhi et al. (2014). "Postural sway in idiopathic rapid eye movement sleep behavior disorder: A potential marker of prodromal Parkinsons disease". In: Brain Research 1559, pp. 26-32. ISSN: 00068993. DOI: 10.1016/j . brainres . 2014. 02 . 040. URL: http : / / www . ncbi . nlm . nih . gov / pubmed/24602694https : / / linkinghub.elsevier.com/retrieve/pii/S0006899314002984.

Chen-Plotkin, Alice $S$ et al. (2018). "Finding useful biomarkers for Parkinson's disease." In: Science translational medicine 10.454, eaam6003. ISSN: 1946-6242. DOI: 10.1126/scitranslmed.aam6003. URL: http://www.ncbi.nlm.nih.gov/pubmed/ 30111645http://www . pubmedcentral . nih.gov/articlerender.fcgi?artid= PMC6097233.

Cooper, J A et al. (1994). "Slowed central processing in simple and go/no-go reaction time tasks in Parkinson's disease." In: Brain : a journal of neurology $117(\mathrm{Pt} 3)$, pp. 517-29. ISSN: 0006-8950. URL: http : / / www . ncbi . nlm . nih . gov/pubmed/ 8032862.

Crossing the Quality Chasm (2001). Washington, D.C.: National Academies Press. ISBN: 978-0-309-07280-9. DOI: 10 . 17226/10027. URL: http : / www . nap . edu/catalog/ 10027.

Dai, Houde, Haijun Lin, and Tim C Lueth (2015a). “Quantitative assessment of parkinsonian bradykinesia based on an inertial measurement unit". In: BioMedical Engineering OnLine 14.1, p. 68. ISSN: 1475-925X. DOI: 10.1186/s12938-015-0067-8. URL: http://www . biomedical-engineering-online.com/content/14/1/68.

- (2015b). "Quantitative assessment of parkinsonian bradykinesia based on an inertial measurement unit". In: BioMedical Engineering OnLine 14.1, p. 68. ISSN: 
1475-925X. DOI: 10 . 1186/s12938-015-0067-8. URL: http : / www . biomedicalengineering-online.com/content/14/1/68.

Das, Rajat Kumar, Sudipta Mukhopadhyay, and Puranjoy Bhattacharya (2014). "User Authentication Based on Keystroke Dynamics". In: IETE Journal of Research 60.3, pp. 229-239. ISSN: 0377-2063. DOI: 10.1080/03772063 . 2014 .914686. URL: http: //www. tandfonline.com/doi/abs/10.1080/03772063.2014.914686.

Del Din, Silvia et al. (2016). "Free-living monitoring of Parkinson's disease: Lessons from the field". In: Movement Disorders 31.9, pp. 1293-1313. ISSN: 08853185. DOI: 10.1002/mds.26718. URL: http://www.ncbi.nlm.nih.gov/pubmed/27452964http: //doi.wiley.com/10.1002/mds. 26718.

Delenclos, Marion et al. (2016). "Biomarkers in Parkinson's disease: Advances and strategies". In: Parkinsonism \& Related Disorders 22, S106-S110. ISSN: 13538020. DOI: 10 . 1016/j . parkreldis . 2015 . 09 .048. URL: http: //www . ncbi .nlm.nih . gov/pubmed/26439946http: / / ww . pubmedcentral . nih.gov/articlerender . fcgi? artid=PMC5120398https : / / linkinghub.elsevier.com/retrieve/pii / S1353802015004393.

DeLong, E R, D M DeLong, and D L Clarke-Pearson (1988). “Comparing the areas under two or more correlated receiver operating characteristic curves: a nonparametric approach." In: Biometrics 44.3, pp. 837-45. ISSN: 0006-341X. URL: http : //www.ncbi.nlm.nih.gov/pubmed/3203132.

Desrosiers, J. et al. (1995). "The Purdue Pegboard Test: Normative data for people aged 60 and over". In: Disability and Rehabilitation 17.5, pp. 217-224. ISSN: 09638288. DOI: 10 .3109/09638289509166638. URL: http: //www . tandfonline . com/ doi/full/10.3109/09638289509166638.

Dorsey, E. R. et al. (2007). "Projected number of people with Parkinson disease in the most populous nations, 2005 through 2030". In: Neurology 68.5, pp. 384-386. ISSN: 0028-3878. DOI: 10 . $1212 / 01$ wnl . 0000247740 . 47667 . 03. URL: http : / / www . ncbi . nlm . nih . gov / pubmed / 17082464http : / / www . neurology . org / cgi/doi/10.1212/01.wnl.0000247740.47667.03.

Dorsey, E. Ray et al. (2016). "Moving Parkinson care to the home". In: Movement Disorders 31.9, pp. 1258-1262. ISSN: 08853185. DOI: 10 . 1002 /mds . 26744. URL: http://www.ncbi.nlm.nih.gov/pubmed/27501323http://www.pubmedcentral. nih.gov/articlerender.fcgi?artid=PMC5014631http://doi . wiley . com/10 . $1002 / \mathrm{mds} .26744$.

Dorsey, E Ray et al. (2017a). “The First Frontier: Digital Biomarkers for Neurodegenerative Disorders". In: Digital Biomarkers 1.1, pp. 6-13. ISSN: 2504-110X. DOI: 10. 1159/000477383. URL: https://www .karger.com/Article/FullText/477383.

- (2017b). "The First Frontier: Digital Biomarkers for Neurodegenerative Disorders". In: Digital Biomarkers 1.1, pp. 6-13. ISSN: 2504-110X. DOI: 10.1159/000477383. URL: https://www . karger. com/Article/FullText/477383.

Doty, Richard L. (2012). "Olfactory dysfunction in Parkinson disease". In: Nature Reviews Neurology 8.6, pp. 329-339. ISSN: 1759-4758. DOI: 10 . $1038 / \mathrm{nrneurol} \mathrm{.}$ 2012.80. URL: http://www.ncbi.nlm.nih.gov/pubmed/22584158http: //www . nature.com/articles/nrneurol.2012.80.

Ellis, Robert J et al. (2015). "A Validated Smartphone-Based Assessment of Gait and Gait Variability in Parkinson's Disease". In: PLOS ONE 10.10. Ed. by Jessica Adrienne Grahn, e0141694. ISSN: 1932-6203. DOI: 10 . 1371/ journal . pone . 0141694. URL: https://dx.plos.org/10.1371/journal.pone. 0141694 .

Eltahir, W E et al. (2004). "Dynamic keystroke analysis using AR model". In: 2004 IEEE International Conference on Industrial Technology, 2004. IEEE ICIT '04. Vol. 3, pp. 1555-1560. 
Eltoukhy, Moataz et al. (2017). "Prediction of ground reaction forces for Parkinson's disease patients using a kinect-driven musculoskeletal gait analysis model". In: Medical Engineering \& Physics 50, pp. 75-82. ISSN: 13504533. DOI: $10.1016 / \mathrm{j}$. medengphy . 2017 . 10 . 004. URL: http : / / www . ncbi.nlm . nih.gov/pubmed/ 29102274https://linkinghub.elsevier.com/retrieve/pii/S135045331730259X.

Erkinjuntti, Timo et al. (1997). "The Effect of Different Diagnostic Criteria on the Prevalence of Dementia". In: New England Journal of Medicine 337.23, pp. 16671674. ISSN: 0028-4793. DOI: 10 . 1056/NEJM199712043372306. URL: http : / / www . nejm.org/doi/abs/10.1056/NEJM199712043372306.

Erro, Roberto et al. (2013). "The heterogeneity of early Parkinson's disease: a cluster analysis on newly diagnosed untreated patients." In: PloS one 8.8, e70244. ISSN: 1932-6203. DOI: 10 . 1371 / journal . pone . 0070244. URL: http : / / ww . ncbi .nlm . nih . gov/pubmed/23936396http: / / www . pubmedcentral . nih . gov/ articlerender.fcgi?artid=PMC3731357.

Espay, Alberto J. et al. (2016). “Technology in Parkinson's disease: Challenges and opportunities". In: Movement Disorders 31.9, pp. 1272-1282. ISSN: 08853185. DOI: 10.1002/mds . 26642. URL: http://doi.wiley.com/10.1002/mds . 26642.

Fahn, Stanley, Group, and the Parkinson Study (2005). "Does levodopa slow or hasten the rate of progression of Parkinson's disease?" In: Journal of Neurology 252.S4, pp. iv37-iv42. ISSN: 0340-5354. DOI: 10 . 1007/s00415-005-4008-5. URL: http: //link. springer.com/10.1007/s00415-005-4008-5.

Fahn, Stanley et al. (2004). "Levodopa and the Progression of Parkinson's Disease". In: New England Journal of Medicine 351.24, pp. 2498-2508. ISSN: 0028-4793. DOI: 10 . 1056 / NEJMoa033447. URL: http : / / www . ncbi . nlm . nih . gov / pubmed / 15590952http://www.nejm.org/doi/abs/10.1056/NEJMoa033447.

Fawcett, Tom (2006). "An introduction to ROC analysis". In: Pattern Recognition Letters 27.8, pp. 861-874. ISSN: 01678655. DOI: 10.1016/ j · patrec. 2005 . 10.010. URL: http://linkinghub.elsevier.com/retrieve/pii/S016786550500303X.

Fekete, R. and J. Jankovic (2011). "Revisiting the relationship between essential tremor and Parkinson's disease". In: Movement Disorders 26.3, pp. 391-398. ISSN: 08853185. DOI: 10 . 1002 /mds . 23512. URL: http : / / www . ncbi . nlm . nih . gov / pubmed / 21462256http://doi.wiley.com/10.1002/mds . 23512.

Fereshtehnejad, Seyed-Mohammad et al. (2017). “Clinical criteria for subtyping Parkinson's disease: biomarkers and longitudinal progression". In: Brain 140.7, pp. 19591976. ISSN: 0006-8950. DOI: 10 . 1093/brain/awx118. URL: http : / www . ncbi . nlm.nih.gov/pubmed/28549077https : / / academic . oup . com/brain/articlelookup/doi/10.1093/brain/awx118.

Ferreira, J. J. et al. (2013). "Summary of the recommendations of the EFNS/MDS-ES review on therapeutic management of Parkinson's disease". In: European Journal of Neurology 20.1, pp. 5-15. ISSN: 13515101. DOI: 10 .1111/ j .1468-1331 . 2012 . 03866.x. URL: http://www.ncbi.nlm.nih.gov/pubmed/23279439http://doi . wiley.com/10.1111/j.1468-1331.2012.03866.x.

Ferreira, Joaquim J. et al. (2015). “Quantitative home-based assessment of Parkinson's symptoms: The SENSE-PARK feasibility and usability study". In: BMC Neurology 15.1, p. 89. ISSN: 1471-2377. DOI: $10.1186 /$ s12883-015-0343-z. URL: http://www.ncbi.nlm.nih.gov/pubmed/26059091http://www.pubmedcentral . nih.gov/articlerender.fcgi?artid=PMC4460963https://bmcneurol . biomedcentral . com/articles/10.1186/s12883-015-0343-z.

Filippeschi, Alessandro et al. (2017). "Survey of Motion Tracking Methods Based on Inertial Sensors: A Focus on Upper Limb Human Motion." In: Sensors (Basel, Switzerland) 17.6. ISSN: 1424-8220. DOI: 10 .3390/s17061257. URL: http: / /ww . 
ncbi .nlm . nih . gov/pubmed/28587178http: //www . pubmedcentral . nih . gov/ articlerender.fcgi?artid=PMC5492902.

Fisher, James M. et al. (2016). “Unsupervised home monitoring of Parkinson's disease motor symptoms using body-worn accelerometers". In: Parkinsonism $\mathcal{E} R e-$ lated Disorders 33, pp. 44-50. ISSN: 13538020. DOI: 10.1016/j . parkreldis . 2016. 09. 009. URL: http : / / www . ncbi .nlm . nih . gov / pubmed / 27637282https : / / linkinghub.elsevier.com/retrieve/pii/S1353802016303340.

Forsaa, E. B. et al. (2010). "What predicts mortality in Parkinson disease?: A prospective population-based long-term study". In: Neurology 75.14, pp. 1270-1276. ISSN: 0028-3878. DOI: 10 .1212/WNL . 0b013e3181f61311. URL: http://www . ncbi .nlm . nih.gov/pubmed/20921512http://www . neurology . org/cgi/doi/10.1212/WNL . Ob013e3181f61311.

Fuente-Fernández, Raúl de la (2013). “Imaging of Dopamine in PD and Implications for Motor and Neuropsychiatric Manifestations of PD." In: Frontiers in neurology 4, p. 90. ISSN: 1664-2295. DOI: 10 .3389/fneur . 2013 . 00090. URL: http : / www . ncbi .nlm . nih . gov/pubmed/23847589http: //www . pubmedcentral . nih . gov/ articlerender.fcgi?artid=PMC3705195.

Gaenslen, Alexandra et al. (2011). “The patients' perception of prodromal symptoms before the initial diagnosis of Parkinson's disease." In: Movement disorders : official journal of the Movement Disorder Society 26.4, pp. 653-658. ISSN: 1531-8257. DOI: 10 . 1002 /mds . 23499. URL: http : / / www . ncbi . nlm . nih . gov/pubmed / 21370256http: //www . pubmedcentral . nih.gov/articlerender.fcgi? artid= PMC3130930.

Gamboa, Javier et al. (1997). “Acoustic voice analysis in patients with Parkinson's disease treated with dopaminergic drugs". In: Journal of Voice 11.3, pp. 314-320. ISSN: 0892-1997. DOI: 10 . 1016/S0892-1997 (97) 80010 - 0. URL: https : / /www . sciencedirect.com/science/article/pii/S0892199797800100.

Gao, Chao et al. (2018). "Model-based and Model-free Machine Learning Techniques for Diagnostic Prediction and Classification of Clinical Outcomes in Parkinson's Disease." In: Scientific reports 8.1, p. 7129. ISSN: 2045-2322. DOI: 10.1038/s41598018-24783-4. URL: http : / /ww . ncbi .nlm . nih . gov/pubmed/29740058http : //www . pubmedcentral . nih.gov/articlerender.fcgi?artid=PMC5940671.

Garcia-Borreguero, Diego et al. (2002). “Decreased phasic EMG activity during rapid eye movement sleep in treatment-naïve Parkinson's disease: Effects of treatment with levodopa and progression of illness". In: Movement Disorders 17.5, pp. 934941. ISSN: 08853185. DOI: $10.1002 / \mathrm{mds}$. 10233. URL: http://www.ncbi.nlm.nih. gov/pubmed/12360542http://doi.wiley.com/10.1002/mds. 10233.

Gelb, Douglas J. et al. (1999). "Diagnostic Criteria for Parkinson Disease". In: Archives of Neurology 56.1, p. 33. ISSN: 0003-9942. DOI: 10 .1001/archneur . 56 .1 . 33. URL: http : / / archneur . jamanetwork . com/article . aspx?doi=10 .1001/archneur . 56.1 .33$.

Giancardo, L. et al. (2015). "Psychomotor Impairment Detection via Finger Interactions with a Computer Keyboard During Natural Typing". In: Scientific Reports 5.November, p. 9678. DOI: 10.1038/srep09678.

Giancardo, L et al. (2016). "Computer keyboard interaction as an indicator of early Parkinson's disease." In: Scientific reports 6.6, p. 34468. ISSN: 2045-2322. DOI: 10. 1038/srep34468. URL: http://www.ncbi.nlm.nih.gov/pubmed/27703257http: //www . pubmedcentral .nih.gov/articlerender.fcgi?artid=PMC5050498.

Gibb, W R and A J Lees (1988). "The relevance of the Lewy body to the pathogenesis of idiopathic Parkinson's disease." In: Journal of neurology, neurosurgery, and psychiatry 51.6, pp. 745-52. ISSN: 0022-3050. URL: http: //www .ncbi .nlm.nih.gov/ 
pubmed/2841426http: //www . pubmedcentral . nih.gov/articlerender . fcgi? artid=PMC1033142.

Ginis, Pieter et al. (2016). "Feasibility and effects of home-based smartphone-delivered automated feedback training for gait in people with Parkinson's disease: A pilot randomized controlled trial". In: Parkinsonism \& Related Disorders 22, pp. 28 34. ISSN: 13538020. DOI: 10 . $1016 / \mathrm{j}$. parkreldis . 2015 . 11 .004. URL: http : //www . ncbi.nlm.nih.gov/pubmed/26777408https : //linkinghub . elsevier . com/retrieve/pii/S1353802015300274.

Giuffrida, Joseph P. et al. (2009). "Clinically deployable Kinesia ${ }^{\mathrm{TM}}$ technology for automated tremor assessment". In: Movement Disorders 24.5, pp. 723-730. ISSN: 08853185. DOI: 10 . 1002 /mds . 22445. URL: http : / /www . ncbi . nlm . nih . gov / pubmed/19133661http://doi.wiley.com/10.1002/mds.22445.

Goetz, Christopher G. et al. (2004). " $<$ i $>$ Movement $</$ i $>$ Disorder Society Task Force report on the Hoehn and Yahr staging scale: Status and recommendations The $<\mathrm{i}>$ Movement $</ \mathrm{i}>$ Disorder Society Task Force on rating scales for Parkinson's disease". In: Movement Disorders 19.9, pp. 1020-1028. ISSN: 08853185. DOI: 10 . 1002/mds . 20213. URL: http://www .ncbi.nlm.nih.gov/pubmed/15372591http: //doi.wiley.com/10.1002/mds. 20213.

Goetz, Christopher G. et al. (2008). "Movement Disorder Society-sponsored revision of the Unified Parkinson's Disease Rating Scale (MDS-UPDRS): Scale presentation and clinimetric testing results". In: Movement Disorders 23.15, pp. 2129-2170. ISSN: 08853185. DOI: 10.1002/mds.22340. URL: http://www.ncbi.nlm.nih.gov/ pubmed/19025984http://doi.wiley.com/10.1002/mds.22340.

Goodfellow, Ian, Yoshua Bengio, and Aaron Courville. Deep learning, p. 775. ISBN: 9780262035613. URL: https://mitpress .mit.edu/books/deep-learning.

Grosset, Donald. (2009). Parkinson's disease. Manson, p. 176. ISBN: 1840765178. URL: https://books.google.com/books/about/Parkinson_s_Disease.html?id=KEXhIJ6-sYC\&source=kp_cover.

Haas, Brian R, Tessandra H Stewart, and Jing Zhang (2012). "Premotor biomarkers for Parkinson's disease - a promising direction of research". In: Translational Neurodegeneration 1.1, p. 11. ISSN: 2047-9158. DOI: 10 . $1186 / 2047-9158-1$ 11. URL: http : / / www . ncbi . nlm . nih . gov/ pubmed / 23211054http : / / www . pubmedcentral . nih . gov / articlerender . fcgi ? artid= PMC3514104http : / / translationalneurodegeneration . biomedcentral . com/articles/10 . 1186/ 2047-9158-1-11.

Haddad, Fatma et al. (2017). "Dopamine and Levodopa Prodrugs for the Treatment of Parkinson's Disease." In: Molecules (Basel, Switzerland) 23.1. ISSN: 1420-3049. DOI: 10 . 3390 /molecules23010040. URL: http : / / www . ncbi .nlm . nih . gov / pubmed/29295587http: //www . pubmedcentral .nih.gov/articlerender .fcgi? artid=PMC5943940.

Haehner, Antje, Thomas Hummel, and Heinz Reichmann (2009). “Olfactory dysfunction as a diagnostic marker for Parkinson's disease". In: Expert Review of Neurotherapeutics 9.12, pp. 1773-1779. ISSN: 1473-7175. DOI: 10 . 1586/ern . 09. 115. URL: http : //www . ncbi . nlm . nih . gov/pubmed/19951136http : / / www . tandfonline.com/doi/full/10.1586/ern.09.115.

- (2014). "A clinical approach towards smell loss in Parkinson's disease." In: Journal of Parkinson's disease 4.2, pp. 189-95. ISSN: 1877-718X. DOI: 10 . 3233 / JPD 130278. URL: http://www.ncbi.nlm.nih.gov/pubmed/24322062.

Haring, Kristen. (2007). Ham radio's technical culture. MIT Press, p. 220. ISBN: 9780262083553. URL: https : / / books . google . com / books ? id=2ly7rDKeGZsC \& pg = PA23\# v = onepage\&q\&f=false. 
Hauser, Robert A. et al. (2009). "Long-term outcome of early versus delayed rasagiline treatment in early Parkinson's disease". In: Movement Disorders 24.4, pp. 564573. ISSN: 08853185. DOI: $10.1002 / \mathrm{mds}$. 22402. URL: http: //www .ncbi.nlm.nih. gov/pubmed/19086083http://doi.wiley.com/10.1002/mds. 22402.

Heemels, Marie-Thérèse (2016). "Neurodegenerative diseases". In: Nature 539.7628, p. 179. ISSN: 0028-0836. DOI: 10 . 1038/539179a. URL: http://www . nature . com/ doifinder/10.1038/539179a.

Heikkilä, V M et al. (1998). "Decreased driving ability in people with Parkinson's disease." In: Journal of neurology, neurosurgery, and psychiatry 64.3, pp. 325-330. ISSN: 0022-3050. DOI: 10.1136/JNNP. 64.3.325. URL: http://www .ncbi.nlm.nih. gov / pubmed / 9527142http : / / www . pubmedcentral . nih . gov/articlerender . fcgi?artid=PMC2170019.

Heinzel, Sebastian et al. (2016). “Prodromal Markers in Parkinson's Disease: Limitations in Longitudinal Studies and Lessons Learned". In: Frontiers in Aging Neuroscience 8 , p. 147. ISSN: 1663-4365. DOI: 10.3389/fnagi . 2016.00147. URL: http: //www .ncbi.nlm.nih.gov/pubmed/27445791http://www . pubmedcentral.nih . gov/articlerender . fcgi ? artid=PMC4916171http : / / journal . frontiersin . org/Article/10.3389/fnagi.2016.00147/abstract.

Heldman, Dustin A et al. (2014). "Clinician versus machine: reliability and responsiveness of motor endpoints in Parkinson's disease." In: Parkinsonism $\mathcal{E}$ related disorders 20.6, pp. 590-5. ISSN: 1873-5126. DOI: 10 . 1016/ j . parkreldis . 2014. 02 . 022. URL: http: //www . ncbi.nlm.nih.gov/pubmed/24661464http: //www . pubmedcentral .nih.gov/articlerender.fcgi?artid=PMC4028404.

Heldman, Dustin A et al. (2017). “Telehealth Management of Parkinson's Disease Using Wearable Sensors: An Exploratory Study". In: Digital Biomarkers 1.1, pp. 4351. ISSN: 2504-110X. DOI: 10.1159/000475801. URL: http://www.ncbi.nlm.nih. gov/pubmed/29725667http : / /ww . pubmedcentral . nih.gov/articlerender . fcgi?artid=PMC5927622https://www . karger. com/Article/FullText/475801.

Hely, M A et al. (1999). "The sydney multicentre study of Parkinson's disease: progression and mortality at 10 years." In: Journal of neurology, neurosurgery, and psychiatry 67.3, pp. 300-7. ISSN: 0022-3050. URL: http: / /www . ncbi.nlm.nih.gov/ pubmed/10449550http: //www . pubmedcentral.nih.gov/articlerender.fcgi? artid=PMC1736543.

Hess, Christopher W and Michael S Okun (2016). "Diagnosing Parkinson Disease". In: Continuum (Minneapolis, Minn.) 22.4 Movement Disorders, 1047-1063. ISSN: 1080-2371. DOI: 10 . 1212/ con . 0000000000000345. URL: https : // doi . org/10 . 1212/CON. 0000000000000345.

Hird, Nick, Samik Ghosh, and Hiroaki Kitano (2016). "Digital health revolution: perfect storm or perfect opportunity for pharmaceutical R\&amp;D?" In: Drug Discovery Today 21.6, pp. 900-911. ISSN: 1359-6446. DOI: 10 . 1016 / J . DRUDIS . 2016.01.010. URL: https://www. sciencedirect.com/science/article/pii/ S1359644616000301.

Ho, Aileen K. et al. (1998). "Speech impairment in a large sample of patients with Parkinson's disease." In: Behavioural neurology 11.3, pp. 131-137.

Hobart, Jeremy C et al. (2007). "Rating scales as outcome measures for clinical trials in neurology: problems, solutions, and recommendations". In: neurology.thelancet.com 6. DOI: 10.1016/S1474-4422(07) 70290-9. URL: http: //www . med .mcgill.ca/ epidemiology / courses / EPIB654 / Summer2010 / EF / Hobart2007LancetNeurol . pdf.

Hochreiter, Sepp and Jürgen Schmidhuber (1997). "Long Short-Term Memory". In: Neural Computation 9.8, pp. 1735-1780. ISSN: 0899-7667. DOI: 10 . 1162 / neco . 
1997.9.8.1735. URL: http://www . mitpressjournals .org/doi/10.1162/neco. 1997.9.8.1735.

Hoehn, M. and M. Yahr (2011). "Parkinsonism: Onset, progression, and mortality". In: Neurology 77.9, pp. 874-874. ISSN: 0028-3878. DOI: 10.1212/01. wnl.0000405146. 06300 . 91. URL: http : / / www . neurology . org / cgi / doi / 10 . 1212/01 . wnl . 0000405146.06300 .91$.

Holford, Nick H. G. and John G. Nutt (2011). “Interpreting the results of Parkinson's disease clinical trials: Time for a change". In: Movement Disorders 26.4, pp. 569577. ISSN: 08853185. DOI: 10.1002/mds . 23555. URL: http://www.ncbi.nlm.nih . gov/pubmed/21370266http://doi.wiley.com/10.1002/mds. 23555.

Homann, C N et al. (2000). “The Bradykinesia Akinesia Incoordination Test (BRAIN TEST), an objective and user-friendly means to evaluate patients with parkinsonism." In: Movement disorders : official journal of the Movement Disorder Society 15.4, pp. 641-7.

Horak, Fay B and Martina Mancini (2013). "Objective biomarkers of balance and gait for Parkinson's disease using body-worn sensors." In: Movement disorders : official journal of the Movement Disorder Society 28.11, pp. 1544-1551. ISSN: 15318257. DOI: 10.1002/mds . 25684. URL: http://www . ncbi.nlm.nih.gov/pubmed/ 24132842http://www . pubmedcentral . nih.gov/articlerender.fcgi?artid= PMC3927718.

Houde Dai, Houde et al. (2013). "A portable system for quantitative assessment of parkinsonian rigidity". In: 2013 35th Annual International Conference of the IEEE Engineering in Medicine and Biology Society (EMBC). Vol. 2013. IEEE, pp. 65916594. ISBN: 978-1-4577-0216-7. DOI: 10 . 1109/EMBC . 2013 . 6611066. URL: http: //www.ncbi.nlm.nih.gov/pubmed/24111253http: //ieexplore. ieee.org/ document/6611066/.

Hssayeni, Murtadha D, Michelle A Burack, and Behnaz Ghoraani (2016). "Automatic assessment of medication states of patients with Parkinson's disease using wearable sensors". In: 2016 38th Annual International Conference of the IEEE Engineering in Medicine and Biology Society (EMBC). Vol. 2016. IEEE, pp. 6082-6085. ISBN: 9781-4577-0220-4. DOI: 10 . 1109/EMBC . 2016 .7592116. URL: http://www . ncbi . nlm . nih.gov/pubmed/28269640http://ieeexplore. ieee.org/document/7592116/.

Hughes, A J et al. (1992). "What features improve the accuracy of clinical diagnosis in Parkinson's disease: a clinicopathologic study." In: Neurology 42.6, pp. 11421146. ISSN: 0028-3878. DOI: 10 . 1212/WNL . 42 . 6 .1142. URL: http: //www . ncbi . nlm.nih.gov/pubmed/1603339.

Jahanshahi, M et al. (1995). "Self-initiated versus externally triggered movements. I. An investigation using measurement of regional cerebral blood flow with PET and movement-related potentials in normal and Parkinson's disease subjects." In: Brain : a journal of neurology 118 ( Pt 4), pp. 913-33. ISSN: 0006-8950. URL: http: //www.ncbi.nlm.nih.gov/pubmed/7655888.

Jankovic, J (2008). "Parkinson's disease: clinical features and diagnosis". In: Journal of Neurology, Neurosurgery \& Psychiatry 79.4, pp. 368-376. ISSN: 0022-3050. DOI: 10.1136/jnnp. 2007.131045. URL: http: //www .ncbi.nlm.nih.gov/pubmed/ 18344392http://jnnp.bmj.com/cgi/doi/10.1136/jnnp. 2007.131045.

Jankovic, Joseph (2005). “Motor fluctuations and dyskinesias in Parkinson's disease: Clinical manifestations". In: Movement Disorders 20.S11, S11-S16. ISSN: 0885-3185. DOI: 10 . 1002 /mds . 20458. URL: http : / / www . ncbi . nlm . nih . gov / pubmed / 15822109http://doi.wiley.com/10.1002/mds. 20458.

Jankovic, Joseph and L Giselle Aguilar (2008). "Current approaches to the treatment of Parkinson's disease." In: Neuropsychiatric disease and treatment 4.4, pp. 743-757. 
ISSN: 1176-6328. URL: http: //www . ncbi.nlm.nih.gov/pubmed/19043519http: //www. pubmedcentral.nih.gov/articlerender.fcgi?artid=PMC2536542.

Jankovic, Joseph et al. (2007). "Transdermal Rotigotine". In: Archives of Neurology 64.5, p. 676. ISSN: 0003-9942. DOI: 10 . 1001 /archneur . 64 . 5 . 676. URL: http : //www .ncbi.nlm.nih.gov/pubmed/17502466http: //archneur . jamanetwork. com/article.aspx?doi=10.1001/archneur.64.5.676.

Janocha, Katarzyna and Wojciech Marian Czarnecki (2017). “On Loss Functions for Deep Neural Networks in Classification". In: Schedae Informaticae 25, pp. 1-10. ISSN: 20838476. DOI: 10 . 4467/20838476SI . 16 .004 .6185. URL: http: / / arxiv . org/abs/1702.05659.

Jaywant, Abhishek et al. (2016). "Randomized Controlled Trial of a Home-Based Action Observation Intervention to Improve Walking in Parkinson Disease". In: Archives of Physical Medicine and Rehabilitation 97.5, pp. 665-673. ISSN: 00039993. DOI: 10 . 1016 / j . apmr . 2015 . 12 . 029. URL: http : / / www . ncbi . nlm . nih . gov/pubmed/26808782http : / /ww . pubmedcentral . nih . gov/articlerender. fcgi? artid=PMC4844795https : // linkinghub.elsevier.com/retrieve/pii/ S0003999316000368.

Jindrich, Devin L, Aruna D Balakrishnan, and Jack T Dennerlein (2004). “Effects of keyswitch design and finger posture on finger joint kinematics and dynamics during tapping on computer keyswitches". In: Clinical Biomechanics 19.6, pp. 600608. ISSN: 02680033. DOI: $10.1016 / \mathrm{j}$. clinbiomech . 2004 . 03 .003. URL: http: //www .ncbi.nlm.nih.gov/pubmed/15234484https : //linkinghub. elsevier. com/retrieve/pii/S026800330400052X.

Jobbágy, Ákos et al. (2005). "Analysis of finger-tapping movement". In: Journal of Neuroscience Methods 141.1, pp. 29-39. ISSN: 01650270. DOI: 10.1016/j . jneumeth . 2004.05.009. URL: http://www. ncbi.nlm.nih.gov/pubmed/15585286https: //linkinghub.elsevier.com/retrieve/pii/S016502700400202X.

Joyce, Rick and Gopal Gupta (1990). "Identity authentication based on keystroke latencies". In: Communications of the ACM 33.2, pp. 168-176. ISSN: 00010782. DOI: 10.1145/75577 . 75582. URL: http://portal . acm.org/citation. cfm?doid= 75577.75582.

Keystroke Dynamics. URL: http : / / www . biometric - solutions . com/keystroke dynamics.html.

Kieburtz, Karl and C. Warren Olanow (2015). "Advances in clinical trials for movement disorders". In: Movement Disorders 30.11, pp. 1580-1587. ISSN: 08853185. DOI: 10 . 1002 /mds . 26371. URL: http : / / www . ncbi . nlm . nih . gov / pubmed / 26307591http://doi.wiley.com/10.1002/mds.26371.

Kieburtz, Karl and Parkinson Study Group PramiBID Investigators (2011). "Twicedaily, low-dose pramipexole in early Parkinson's disease: A randomized, placebocontrolled trial". In: Movement Disorders 26.1, pp. 37-44. ISSN: 08853185. DOI: 10. 1002/mds . 23396. URL: http://www .ncbi.nlm.nih.gov/pubmed/20925067http: //doi.wiley.com/10.1002/mds.23396.

Kieburtz, Karl et al. (2015). "Effect of Creatine Monohydrate on Clinical Progression in Patients With Parkinson Disease". In: JAMA 313.6, p. 584. ISSN: 0098-7484. DOI: 10.1001/jama.2015.120. URL: http://www.ncbi .nlm.nih.gov/pubmed/ 25668262http: //www . pubmedcentral . nih . gov/articlerender. fcgi ?artid= PMC4349346http://jama. jamanetwork. com/article. aspx?doi=10.1001/jama . 2015.120.

Kilgore, K L (2015). “Implantable Neuroprostheses for Restoring Function”. In: pp. 1438. 
Kim, Ji-Won et al. (2011). "Quantification of bradykinesia during clinical finger taps using a gyrosensor in patients with Parkinson's disease". In: Medical $\mathcal{E}$ Biological Engineering \& Computing 49.3, pp. 365-371. ISSN: 0140-0118. DOI: 10.1007 / s11517-010-0697-8. URL: http://www.ncbi.nlm.nih.gov/pubmed/21052856http: //link. springer.com/10.1007/s11517-010-0697-8.

Klinger, Evelyne et al. (2006). "Virtual Action Planning in Parkinson's Disease: AControl Study". In: CyberPsychology \& Behavior 9.3, pp. 342-347. ISSN: 1094-9313. DOI: 10.1089/cpb . 2006 .9.342. URL: http://www . liebertpub. com/doi/10 . 1089/ $\mathrm{cpb} .2006 .9 .342$.

Klucken, Jochen et al. (2013). “Unbiased and Mobile Gait Analysis Detects Motor Impairment in Parkinson's Disease". In: PLOS ONE 8.2. Ed. by Mathias Toft, e56956. ISSN: 1932-6203. DOI: 10 . 1371 / journal . pone . 0056956. URL: http : / / www . ncbi . nlm . nih . gov / pubmed / 23431395http : / / www . pubmedcentral . nih.gov/articlerender . fcgi ?artid=PMC3576377https://dx . plos .org/10 . 1371/journal. pone. 0056956.

Kohavi, Ron (1995). "A Study of Cross-Validation and Bootstrap for Accuracy Estimation and Model Selection". In: Appears in the International Joint Conference on Articial Intelligence (IJCAI), pp. 1-7. ISSN: 10450823. DOI: 10 . $1067 / \bmod .2000$. 109031. URL: http://robotics.stanford.edu/ ronnyk.

Kordower, Jeffrey $\mathrm{H}$ et al. (2013). "Disease duration and the integrity of the nigrostriatal system in Parkinson's disease." In: Brain : a journal of neurology 136.Pt 8, pp. 2419-31. ISSN: 1460-2156. DOI: 10 . 1093/brain/awt192. URL: http: / /www . ncbi.nlm.nih.gov/pubmed/23884810http: //www . pubmedcentral . nih . gov/ articlerender.fcgi?artid=PMC3722357.

Kowal, Stacey L. et al. (2013). "The current and projected economic burden of Parkinson's disease in the United States". In: Movement Disorders 28.3, pp.311-318. ISSN: 08853185. DOI: 10 . 1002 /mds . 25292. URL: http : / /www . ncbi . nlm . nih . gov/ pubmed/23436720http://doi.wiley.com/10.1002/mds. 25292.

Kuo, Po-Ling et al. (2006). "Finger joint coordination during tapping". In: Journal of Biomechanics 39.16, pp. 2934-2942. ISSN: 00219290. DOI: 10 . 1016/ j . jbiomech . 2005 . 10.028. URL: http://www . ncbi.nlm.nih.gov/pubmed/16376353https : //linkinghub.elsevier.com/retrieve/pii/S002192900500494X.

Lang, Anthony E (2010). “Clinical trials of disease-modifying therapies for neurodegenerative diseases: the challenges and the future". In: Nature Medicine 16.11, pp. 1223-1226. ISSN: 1078-8956. DOI: 10.1038/nm. 2220. URL: http://www . ncbi . nlm.nih.gov/pubmed/21052078http://www. nature.com/articles/nm. 2220.

- (2011). "A critical appraisal of the premotor symptoms of Parkinson's disease: Potential usefulness in early diagnosis and design of neuroprotective trials". In: Movement Disorders 26.5, pp. 775-783. ISSN: 08853185. DOI: 10.1002/mds . 23609. URL: http://doi.wiley.com/10.1002/mds. 23609.

Lau, Lonneke M L de and Monique M B Breteler (2006). "Epidemiology of Parkinson's disease." English. In: The Lancet. Neurology 5.6, pp. 525-35. DOI: 10 . 1016/ S1474-4422 (06) 70471-9.

Lawton, Michael et al. (2018). “Developing and validating Parkinson's disease subtypes and their motor and cognitive progression". In: Journal of Neurology, Neurosurgery \& Psychiatry, pp. 2018-318337. ISSN: 0022-3050. DOI: 10.1136/jnnp-2018318337. URL: http://jnnp. bmj .com/lookup/doi/10.1136/jnnp-2018-318337.

Ledger, D and D McCaffrey (2014). "Inside wearables: How the science of human behavior change offers the secret to long-Term engagement". In: Endeavour Partners, LLC 93, pp. 36-45. 
Lee, Sunghoon Ivan et al. (2015). "A novel method for assessing the severity of levodopa-induced dyskinesia using wearable sensors". In: 2015 37th Annual International Conference of the IEEE Engineering in Medicine and Biology Society (EMBC). Vol. 2015. IEEE, pp. 8087-8090. ISBN: 978-1-4244-9271-8. DOI: 10 . 1109 / EMBC . 2015.7320270. URL: http://www.ncbi.nlm.nih.gov/pubmed/26738170https: //ieeexplore. ieee.org/document/7320270/.

Lewek, Michael D. et al. (2010). "Arm swing magnitude and asymmetry during gait in the early stages of Parkinson's disease". In: Gait E Posture 31.2, pp. 256-260. ISSN: 09666362. DOI: 10 . 1016/ j gaitpost . 2009 . 10 . 013. URL: http : / / www . ncbi.nlm.nih.gov/pubmed/19945285http://www . pubmedcentral . nih . gov/ articlerender.fcgi ?artid=PMC2818433https : // linkinghub.elsevier .com/ retrieve/pii/S0966636209006481.

Li, Michael H. et al. (2018). "Automated assessment of levodopa-induced dyskinesia: Evaluating the responsiveness of video-based features". In: Parkinsonism $\mathcal{E}$ Related Disorders 53, pp. 42-45. ISSN: 13538020. DOI: 10 . 1016/j · parkreldis . 2018.04.036. URL: http: //www. ncbi.nlm.nih.gov/pubmed/29748112https : //linkinghub.elsevier.com/retrieve/pii/S1353802018302220.

Lim, Shen-Yang and Ai Huey Tan (2018). "Historical perspective: The pros and cons of conventional outcome measures in Parkinson's disease". In: Parkinsonism $\mathcal{E}$ Related Disorders 46, S47-S52. ISSN: 1353-8020. DOI: 10 . 1016 / J . PARKRELDIS . 2017 . 07 . 029. URL: https : //www. sciencedirect. com/science/article/pii/ S1353802017302766.

Little, M.A. et al. (2009). "Suitability of Dysphonia Measurements for Telemonitoring of Parkinson's Disease". In: IEEE Transactions on Biomedical Engineering 56.4, pp. 1015-1022. ISSN: 0018-9294. DOI: 10 . 1109/TBME . 2008 . 2005954. URL: http: //ieeexplore. ieee.org/document/4636708/.

Loi, Michele (2018). "The Digital Phenotype: a Philosophical and Ethical Exploration". In: Philosophy \& Technology, pp. 1-17. ISSN: 2210-5433. DOI: 10 .1007/s13347-0180319-1. URL: http://link. springer.com/10.1007/s13347-018-0319-1.

Ma, M. Joe (2010). "Biopsy Pathology of Neurodegenerative Disorders in Adults". In: Practical Surgical Neuropathology. Elsevier, pp. 551-571. ISBN: 9780443069826. DOI: 10 . 1016 / B978 - 0 - $443-06982-6.00025$ - 0. URL: http : / / linkinghub . elsevier.com/retrieve/pii/B9780443069826000250.

Maetzler, Walter et al. (2013a). "Emerging therapies for gait disability and balance impairment: promises and pitfalls." In: Movement disorders : official journal of the Movement Disorder Society 28.11, pp. 1576-86. DOI: 10.1002/mds . 25682.

Maetzler, Walter et al. (2013b). "Quantitative wearable sensors for objective assessment of Parkinson's disease". In: Movement Disorders 28.12, pp. 1628-1637. ISSN: 08853185. DOI: 10 . 1002 /mds . 25628. URL: http : / /www . ncbi . nlm . nih . gov / pubmed/24030855http://doi.wiley.com/10.1002/mds. 25628.

Magoulas, George D and Andriana Prentza (2001). "Machine Learning in Medical Applications". In: Springer, Berlin, Heidelberg, pp. 300-307. DOI: 10 . $1007 / 3$ 540-44673-7\{\_\}19. URL: http://link. springer.com/10.1007/3-540-446737_19.

Makady, Amr et al. (2017). “What Is Real-World Data? A Review of Definitions Based on Literature and Stakeholder Interviews". In: Value in Health 20.7, pp. 858-865. ISSN: 1098-3015. DOI: 10 . 1016 / J . JVAL . 2017 . 03 . 008. URL: https : / / www . sciencedirect.com/science/article/pii/S1098301517301717.

Marek, Kenneth et al. (2011). "The Parkinson Progression Marker Initiative (PPMI)". In: Progress in Neurobiology 95.4, pp. 629-635. ISSN: 03010082. DOI: $10.1016 / \mathrm{j}$. 
pneurobio.2011.09.005. URL: http://linkinghub.elsevier.com/retrieve/ $\mathrm{pii} / \mathrm{S} 0301008211001651$.

Mariani, Benoit et al. (2013). “On-Shoe Wearable Sensors for Gait and Turning Assessment of Patients With Parkinson's Disease". In: IEEE Transactions on Biomedical Engineering 60.1, pp. 155-158. ISSN: 0018-9294. DOI: 10 . 1109/ TBME . 2012. 2227317. URL: http://ieeexplore. ieee.org/document/6384887/.

Marr, Bernard (2019). How Much Data Do We Create Every Day? The Mind-Blowing Stats Everyone Should Read. URL: https://www . forbes .com/sites/bernardmarr/ 2018/05/21/how-much-data-do-we-create-every-day-the-mind-blowingstats-everyone-should-read/\#4b2e915f60ba.

Marsden, C D (1994). "Problems with long-term levodopa therapy for Parkinson's disease." In: Clinical neuropharmacology 17 Suppl 2, pp. 32-44. ISSN: 0362-5664. URL: http://www.ncbi.nlm.nih.gov/pubmed/9358193.

Marsili, Luca, Giovanni Rizzo, and Carlo Colosimo (2018). “Diagnostic Criteria for Parkinson's Disease: From James Parkinson to the Concept of Prodromal Disease". In: Frontiers in Neurology 9, p. 156. ISSN: 1664-2295. DOI: 10 .3389/fneur . 2018. 00156. URL: http : / /ww . ncbi . nlm . nih . gov/ pubmed/29628907http : //www . pubmedcentral . nih.gov/articlerender.fcgi?artid=PMC5877503http: //journal.frontiersin.org/article/10.3389/fneur.2018.00156/full.

Martínez-Martín, P. et al. (1994). “Unified Parkinson's disease rating scale characteristics and structure". In: Movement Disorders 9.1, pp. 76-83. ISSN: 08853185. DOI: 10. 1002 /mds . 870090112. URL: http : / / www . ncbi . nlm . nih . gov / pubmed / 8139608http://doi.wiley.com/10.1002/mds.870090112.

Martínez-Martín, Pablo et al. (2015). "Parkinson's disease severity levels and MDSUnified Parkinson's Disease Rating Scale." In: Parkinsonism \& related disorders 21.1, pp. 50-54. ISSN: 1873-5126. DOI: 10 . 1016/ j . parkreldis . 2014 . 10 . 026. URL: http://www.ncbi.nlm.nih.gov/pubmed/25466406.

Matarazzo, Michele et al. (2019). "Remote Monitoring of Treatment Response in Parkinson's Disease: The Habit of Typing on a Computer". In: Movement Disorders. ISSN: 08853185. DOI: 10 . 1002/mds . 27772. URL: http: //www . ncbi .nlm . nih.gov/pubmed/31211469http://doi.wiley.com/10.1002/mds. 27772.

McCarney, Rob et al. (2007a). "The Hawthorne Effect: a randomised, controlled trial." In: BMC medical research methodology 7, p. 30. ISSN: 1471-2288. DOI: 10.1186/14712288 - 7 - 30. URL: http : / / www . ncbi . nlm . nih . gov / pubmed / 17608932http : //www.pubmedcentral.nih.gov/articlerender.fcgi?artid=PMC1936999.

- (2007b). "The Hawthorne Effect: a randomised, controlled trial". In: BMC Medical Research Methodology 7.1, p. 30. ISSN: 1471-2288. DOI: 10 . 1186/1471-2288-730. URL: http : / / www . ncbi . nlm . nih . gov/pubmed/17608932http : / / www . pubmedcentral . nih . gov / articlerender . fcgi ? artid= PMC1936999http : / / bmcmedresmethodol. biomedcentral . com/articles/10.1186/1471-2288-7-30.

McGhee, David J M et al. (2013). "A systematic review of biomarkers for disease progression in Parkinson's disease". In: BMC Neurology 13.1, p. 35. ISSN: 14712377. DOI: 10.1186/1471-2377-13-35. URL: http: //bmcneurol . biomedcentral . com/articles/10.1186/1471-2377-13-35.

Meister, Sven, Wolfgang Deiters, and Stefan Becker (2016). "Digital health and digital biomarkers - enabling value chains on health data". In: Current Directions in Biomedical Engineering 2.1, pp. 577-581. ISSN: 2364-5504. DOI: 10 . 1515 / cdbme2016 -0128. URL: https : / www . degruyter . com/view/j/cdbme . 2016 . 2 . issue1/cdbme-2016-0128/cdbme-2016-0128.xml.

Memar, Sara et al. (2018). “Quantification of whole-body bradykinesia in Parkinson's disease participants using multiple inertial sensors". In: Journal of the Neurological 
Sciences 387, pp. 157-165. ISSN: 0022510X. DOI: $10.1016 / \mathrm{j}$. jns . 2018.02 .001$. URL: http://www .ncbi .nlm.nih.gov/pubmed/29571855https://linkinghub . elsevier.com/retrieve/pii/S0022510X18300601.

Mendes, Felipe Augusto dos Santos et al. (2012). "Motor learning, retention and transfer after virtual-reality-based training in Parkinson's disease - effect of motor and cognitive demands of games: a longitudinal, controlled clinical study". In: Physiotherapy 98.3, pp. 217-223. ISSN: 00319406. DOI: $10.1016 / \mathrm{j} \cdot$ physio . 2012.06.001. URL: http://www . ncbi.nlm.nih. gov/pubmed/22898578https : //linkinghub.elsevier.com/retrieve/pii/S003194061200051X.

Mera, T. O., M. A. Burack, and J. P. Giuffrida (2012). "Quantitative assessment of levodopa-induced dyskinesia using automated motion sensing technology". In: 2012 Annual International Conference of the IEEE Engineering in Medicine and Biology Society. Vol. 2012. IEEE, pp. 154-157. ISBN: 978-1-4577-1787-1. DOI: 10.1109/ EMBC. 2012.6345894. URL: http://www.ncbi.nlm.nih.gov/pubmed/23365855http: //ieeexplore.iee. org/document/6345894/.

Merola, Aristide et al. (2018). "Technology-based assessment of motor and nonmotor phenomena in Parkinson disease". In: Expert Review of Neurotherapeutics 18.11, pp. 825-845. ISSN: 1473-7175. DOI: 10 . $1080 / 14737175$. 2018 . 1530593. URL: https://www.tandfonline.com/doi/full/10.1080/14737175.2018.1530593.

Miller, Diane B and James P O'Callaghan (2015). “Biomarkers of Parkinson's disease: present and future." In: Metabolism: clinical and experimental 64.3 Suppl 1, pp. 406. ISSN: 1532-8600. DOI: 10 .1016/ j . metabol . 2014 . 10 . 030. URL: http: //www . ncbi .nlm.nih . gov/pubmed/25510818http : / / ww . pubmedcentral . nih . gov/ articlerender.fcgi?artid=PMC4721253.

Mirek, Elzbieta, Monika Rudzińska, and Andrzej Szczudlik (2007). “The assessment of gait disorders in patients with Parkinson's disease using the three-dimensional motion analysis system Vicon." In: Neurologia i neurochirurgia polska 41.2, pp. 12833. ISSN: 0028-3843. URL: http://www.ncbi.nlm.nih.gov/pubmed/17530574.

Mirelman, A et al. (2011). “Virtual Reality for Gait Training: Can It Induce Motor Learning to Enhance Complex Walking and Reduce Fall Risk in Patients With Parkinson's Disease?" In: The Journals of Gerontology Series A: Biological Sciences and Medical Sciences 66A.2, pp. 234-240. ISSN: 1079-5006. DOI: 10.1093/gerona/ glq201. URL: https : / / academic . oup . com / biomedgerontology / article lookup/doi/10.1093/gerona/glq201.

Miyasaki, J. M. et al. (2002). "Practice parameter: Initiation of treatment for Parkinson's disease: An evidence-based review: Report of the Quality Standards Subcommittee of the American Academy of Neurology". In: Neurology 58.1, pp. 1117. ISSN: 0028-3878. DOI: 10.1212/WNL . 58.1.11. URL: http: //www . neurology . org/cgi/doi/10.1212/WNL.58.1.11.

Monica Anderson and Andrew Perrin (2017). "Technology Use Among Seniors". In: Washington, DC: Pew Research Center for Internet \& Technology. URL: http: //www . pewinternet.org/2017/05/17/technology-use-among-seniors/.

Moore, Steven T. et al. (2007). "Long-term monitoring of gait in Parkinson's disease". In: Gait \& Posture 26.2, pp. 200-207. ISSN: 0966-6362. DOI: 10 . 1016/ J . GAITPOST . 2006.09.011. URL: https://www.sciencedirect.com/science/article/pii/ S0966636206001901.

Moro, Elena et al. (2010). "Long-term results of a multicenter study on subthalamic and pallidal stimulation in Parkinson's disease". In: Movement Disorders 25.5, pp. 578-586. ISSN: 08853185. DOI: $10.1002 / \mathrm{mds}$. 22735. URL: http://www . ncbi . nlm.nih.gov/pubmed/20213817http://doi.wiley.com/10.1002/mds. 22735. 
Moskovitch, Robert et al. (2009). "Identity theft, computers and behavioral biometrics". In: 2009 IEEE International Conference on Intelligence and Security Informatics. IEEE, pp. 155-160. ISBN: 978-1-4244-4172-3. DOI: 10 . 1109/ ISI . 2009 . 5137288. URL: http://ieeexplore.ieee.org/document/5137288/.

Nielsen, Tine et al. (2018). "The use of olfactory testing when diagnosing Parkinson's disease - a systematic review." In: Danish medical journal 65.5. ISSN: 2245-1919. URL: http://www.ncbi.nlm.nih.gov/pubmed/29726318.

NINDS NET-PD Investigators (2006). "A randomized, double-blind, futility clinical trial of creatine and minocycline in early Parkinson disease". In: Neurology 66.5, pp. 664-671. ISSN: 0028-3878. DOI: 10.1212/01 . wnl.0000201252.57661.e1. URL: http://www.ncbi.nlm.nih.gov/pubmed/16481597http://www. neurology.org/ cgi/doi/10.1212/01.wnl.0000201252.57661.e1.

- (2007). "A randomized clinical trial of coenzyme Q10 and GPI-1485 in early Parkinson disease". In: Neurology 68.1, pp. 20-28. ISSN: 0028-3878. DOI: 10 . 1212/01. wnl . 0000250355 . 28474 . 8e. URL: http : //www . ncbi .nlm.nih.gov/pubmed/ 17200487http://www . neurology.org/cgi/doi/10.1212/01.wnl.0000250355. $28474.8 \mathrm{e}$.

Noah, Benjamin, Jingwen Li, and Ling Rothrock (2017). "An evaluation of touchscreen versus keyboard/mouse interaction for large screen process control displays". In: Applied Ergonomics 64, pp. 1-13. ISSN: 00036870. DOI: $10.1016 / \mathrm{j}$. apergo.2017.04.015. URL: http://www.ncbi.nlm.nih.gov/pubmed/28610809https: //linkinghub.elsevier.com/retrieve/pii/S0003687017301011.

Noble, Wendy and Mark P Burns (2010). "Challenges in neurodegeneration research." In: Frontiers in psychiatry 1, p. 7. ISSN: 1664-0640. DOI: 10 . 3389 / fpsyt . 2010. 00007. URL: http : //www . ncbi.nlm.nih.gov/pubmed/21423419http: //www . pubmedcentral . nih.gov/articlerender.fcgi?artid=PMC3059656.

Obaidat, M.S. and D.T. Macchiarolo (1993). "An online neural network system for computer access security". In: IEEE Transactions on Industrial Electronics 40.2, pp. 235242. ISSN: 02780046. DOI: 10.1109/41.222645. URL: http: //ieeexplore. ieee. org/document/222645/.

Obeso, Jose A et al. (2010). "Missing pieces in the Parkinson's disease puzzle". In: Nature Medicine 16.6, pp. 653-661. ISSN: 1078-8956. DOI: 10 . 1038/nm. 2165. URL: http: //www . ncbi.nlm.nih.gov/pubmed/20495568http: // www . nature.com/ doifinder/10.1038/nm. 2165.

Okuma, Yasuyuki (2014). "Freezing of gait and falls in Parkinson's disease." In: Journal of Parkinson's disease 4.2, pp. 255-60. ISSN: 1877-718X. DOI: 10 . 3233 / JPD 130282. URL: http://www.ncbi.nlm.nih.gov/pubmed/24577502.

Olanow, C. Warren et al. (2009). "A Double-Blind, Delayed-Start Trial of Rasagiline in Parkinson's Disease". In: New England Journal of Medicine 361.13, pp. 12681278. ISSN: 0028-4793. DOI: 10 . 1056/NEJMoa0809335. URL: http : //www . ncbi . nlm . nih . gov/pubmed/19776408http : / / www . nejm . org/doi /abs / 10 . 1056 / NEJMoa0809335.

Omberg, Larsson et al. (2017). "A large observational study using mobile phone sensors reveals a personalized drug response in patients with Parkinson's Disease (S22.004)". In: Neurology 88.16 Supplement.

Opara, Józef A et al. (2017). "Motor assessment in Parkinson's disease". In: Annals of Agricultural and Environmental Medicine 24.3, pp. 411-415. ISSN: 1232-1966. DOI: 10.5604/12321966.1232774. URL: http://www .ncbi.nlm.nih.gov/pubmed/ $28954481 \mathrm{http}$ : //aaem. pl/abstracted. php?level=5\&ICID=1232774.

Orwat, Carsten, Andreas Graefe, and Timm Faulwasser (2008). “Towards pervasive computing in health care - A literature review". In: BMC Medical Informatics and 
Decision Making 8.1, p. 26. ISSN: 1472-6947. DOI: 10.1186/1472-6947-8-26. URL: http://bmcmedinformdecismak . biomedcentral . com/articles/10.1186/14726947-8-26.

Oung, Qi Wei et al. (2015). "Technologies for Assessment of Motor Disorders in Parkinson's Disease: A Review." In: Sensors (Basel, Switzerland) 15.9, pp. 2171021745. ISSN: 1424-8220. DOI: 10.3390/s150921710. URL: http://www . ncbi.nlm. nih.gov/pubmed/26404288http://www . pubmedcentral.nih.gov/articlerender . fcgi? artid=PMC4610449.

Pagano, Gennaro et al. (2016). "Age at onset and Parkinson disease phenotype." In: Neurology 86.15, pp. 1400-1407. ISSN: 1526-632X. DOI: 10.1212/WNL. 0000000000002461. URL: http://www.ncbi.nlm.nih.gov/pubmed/26865518http://www.pubmedcentral . nih.gov/articlerender.fcgi?artid=PMC4831034.

Pal, P K and M Netravathi (2005). "Management of neurodegenerative disorders: Parkinson's disease and Alzheimer's disease." In: Journal of the Indian Medical Association 103.3, pp. 168-70. ISSN: 0019-5847. URL: http://www .ncbi .nlm.nih . gov/pubmed/16173294.

Palmer, Janice L. et al. (2010). “Unified Parkinson's Disease Rating Scale-Motor Exam: inter-rater reliability of advanced practice nurse and neurologist assessments". In: Journal of Advanced Nursing 66.6, pp. 1382-1387. ISSN: 03092402. DOI: 10.1111/ j . 1365-2648 . 2010.05313.x. URL: http: / /www.ncbi.nlm.nih.gov/pubmed/ 20546368http: //www . pubmedcentral . nih . gov/articlerender.fcgi?artid= PMC2903978http://doi.wiley.com/10.1111/j.1365-2648.2010.05313.x.

Pan-Montojo, Francisco et al. (2012). "Environmental toxins trigger PD-like progression via increased alpha-synuclein release from enteric neurons in mice". In: Scientific Reports 2.1, p. 898. ISSN: 2045-2322. DOI: 10 . 1038/srep00898. URL: http: //www .nature.com/articles/srep00898.

Pandey, Sanjay and Prachaya Srivanitchapoom (2017). "Levodopa-induced Dyskinesia: Clinical Features, Pathophysiology, and Medical Management." In: Annals of Indian Academy of Neurology 20.3, pp. 190-198. ISSN: 0972-2327. DOI: 10 . 4103/ aian. AIAN $\left\{\backslash_{-}\right\} 239\left\{\backslash_{-}\right\} 17$. URL: http : / / www . ncbi.nlm . nih.gov/pubmed / 28904447http://www . pubmedcentral . nih.gov/articlerender.fcgi?artid= PMC5586110.

Papapetropoulos, Spyridon Spyros (2012). "Patient Diaries As a Clinical Endpoint in Parkinson's Disease Clinical Trials". In: CNS Neuroscience \& Therapeutics 18.5, pp. 380-387. ISSN: 17555930. DOI: 10.1111/j .1755-5949 . 2011 .00253 . x. URL: http://doi.wiley.com/10.1111/j.1755-5949.2011.00253.x.

Papapetropoulos, Spyros, Georgia Mitsi, and Alberto J Espay (2015). “Digital Health Revolution: Is it Time for Affordable Remote Monitoring for Parkinsonât ${ }^{\mathrm{TM}_{\mathrm{S}}}$ Disease?" In: Frontiers in Neurology 6, p. 34. ISSN: 1664-2295. DOI: $10.3389 /$ fneur . 2015.00034. URL: http://journal.frontiersin.org/Article/10.3389/fneur. 2015.00034/abstract.

Park, Jaebum et al. (2012). "Changes in multifinger interaction and coordination in Parkinson's disease." In: Journal of neurophysiology 108.3, pp. 915-24. ISSN: 15221598. DOI: 10 . 1152 / jn . 00043 . 2012. URL: http : / /ww . ncbi .nlm . nih . gov/ pubmed/22552184http://www . pubmedcentral . nih.gov/articlerender.fcgi? artid=PMC3424084.

Parkinson's disease: diagnosis and clinical management (2008). Demos, p. 819. ISBN: 9781934559871.

Parzen, Emanuel (2007). “On Estimation of a Probability Density Function and Mode”. In: The Annals of Mathematical Statistics 33, pp. 1065-1076. DOI: 10.2307/2237880. URL: https://www. jstor.org/stable/2237880. 
Pastorino, M et al. (2013). "Wearable sensor network for health monitoring: the case of Parkinson disease". In: Journal of Physics: Conference Series 450.1, p. 12055. ISSN: 1742-6588. DOI: 10 . 1088/1742-6596/450/1/012055. URL: http://stacks . iop . org $/ 1742-6596 / 450 / i=1 / a=012055$ ?key=crossref . b5f283b4d7de3b616e7227fd7155c84d.

Patel, Shyamal et al. (2009). "Monitoring motor fluctuations in patients with Parkinson's disease using wearable sensors." In: IEEE transactions on information technology in biomedicine : a publication of the IEEE Engineering in Medicine and Biology Society 13.6, pp. 864-73. DOI: 10.1109/TITB. 2009. 2033471.

Pavel, Misha et al. (2007). "Continuous Assessment of Gait Velocity in Parkinson's Disease from Unobtrusive Measurements". In: 2007 3rd International IEEE/EMBS Conference on Neural Engineering. IEEE, pp. 700-703. ISBN: 1-4244-0791-5. DOI: 10 . 1109/CNE . 2007 . 369769. URL: http : / i ieeexplore . ieee . org/document/ 4227374/.

Peña-Nogales, Óscar et al. (2019). "Longitudinal Connectomes as a Candidate Progression Marker for Prodromal Parkinson's Disease". In: Frontiers in Neuroscience 12, p. 967. ISSN: 1662-453X. DOI: 10 . 3389/fnins . 2018 .00967. URL: https : // www.frontiersin.org/article/10.3389/fnins.2018.00967/full.

Pérez-López, Carlos et al. (2016). “Dopaminergic-induced dyskinesia assessment based on a single belt-worn accelerometer". In: Artificial Intelligence in Medicine 67, pp. 47-56. ISSN: 09333657. DOI: 10.1016/j . artmed . 2016.01.001. URL: http: //www .ncbi.nlm.nih.gov/pubmed/26831150https : //linkinghub . elsevier. com/retrieve/pii/S0933365716000038.

Perkins, Neil J and Enrique F Schisterman (2006). "The Inconsistency of " Optimal " Cut-points Using Two ROC Based Criteria". In: Am J Epidemiol 163.7, pp. 670675. ISSN: 0002-9262. DOI: 10.1093/aje/kwj063.

Perlmutter, Joel S (2009). "Assessment of Parkinson disease manifestations." In: Current protocols in neuroscience Chapter 10, Unit10.1. ISSN: 1934-8576. DOI: 10.1002/ 0471142301.ns1001s49. URL: http://www.ncbi.nlm.nih.gov/pubmed/19802812http: //www.pubmedcentral.nih.gov/articlerender.fcgi?artid=PMC2897716.

"Personal identification apparatus" (1983). In: URL: https://patents .google.com/ patent/US4621334A/en.

Perumal, Shyam V and Ravi Sankar (2016). “Gait and tremor assessment for patients with Parkinson's disease using wearable sensors". In: ICT Express 2.4, pp. 168174. ISSN: 2405-9595. DOI: 10 . 1016/ J . ICTE . 2016 . 10 . 005. URL: https : / www . sciencedirect.com/science/article/pii/S2405959516301382.

Peto, V, C Jenkinson, and R Fitzpatrick (1998). "PDQ-39: a review of the development, validation and application of a Parkinson's disease quality of life questionnaire and its associated measures." In: Journal of neurology 245 Suppl 1, pp. 10-4. ISSN: 0340-5354. URL: http://www .ncbi.nlm.nih.gov/pubmed/9617716.

Pfeiffer, Ronald F. (2016). "Non-motor symptoms in Parkinson's disease”. In: Parkinsonism \& Related Disorders 22, S119-S122. ISSN: 1353-8020. DOI: 10 . 1016 / J . PARKRELDIS . 2015 .09.004. URL: https : / /www . sciencedirect. com/science/ article/pii/S135380201500379X.

Poewe, W and G Wenning (2002). "The differential diagnosis of Parkinson's disease." In: European journal of neurology 9 Suppl 3, pp. 23-30. ISSN: 1351-5101. URL: http://www.ncbi.nlm.nih.gov/pubmed/12464118.

Poewe, W. et al. (2011). "Extended-release pramipexole in early Parkinson disease: A 33-week randomized controlled trial". In: Neurology 77.8, pp. 759-766. ISSN: 0028-3878. DOI: 10 .1212/WNL . Ob013e31822affb0. URL: http://www . ncbi . nlm . nih.gov/pubmed/21832218http://www . neurology.org/cgi/doi/10.1212/WNL . ob013e31822affb0. 
Poewe, Werner et al. (2017). "Parkinson disease". In: Nature Reviews Disease Primers 3, p. 17013. ISSN: 2056-676X. DOI: 10 . 1038/nrdp. 2017 . 13. URL: http: / /www . nature.com/articles/nrdp201713.

Postuma, R. B. et al. (2012). "How does parkinsonism start? Prodromal parkinsonism motor changes in idiopathic REM sleep behaviour disorder". In: Brain 135.6, pp. 1860-1870. ISSN: 0006-8950. DOI: 10.1093/brain/aws093. URL: http://www . ncbi .nlm . nih . gov/ pubmed / 22561644https : / / academic . oup . com/brain / article-lookup/doi/10.1093/brain/aws093.

Postuma, Ronald B et al. (2015). "Parkinson risk in idiopathic REM sleep behavior disorder: preparing for neuroprotective trials." In: Neurology 84.11, pp. 11041113. ISSN: 1526-632X. DOI: 10.1212/WNL . 0000000000001364 . URL: http: //www . ncbi .nlm . nih . gov/pubmed / 25681454http : / /ww . pubmedcentral . nih . gov/ articlerender.fcgi?artid=PMC4371408.

Prashanth, R. et al. (2014). "Parkinson's disease detection using olfactory loss and REM sleep disorder features". In: 2014 36th Annual International Conference of the IEEE Engineering in Medicine and Biology Society. IEEE, pp. 5764-5767. ISBN: 9781-4244-7929-0. DOI: 10 . 1109 / EMBC . 2014 . 6944937. URL: http : / i ieeexplore . ieee.org/document/6944937/.

Ramaker, Claudia et al. (2002). "Systematic evaluation of rating scales for impairment and disability in Parkinson's disease". In: Movement Disorders 17.5, pp. 867876. ISSN: 0885-3185. DOI: 10.1002/mds .10248. URL: http://doi .wiley . com/10. $1002 / \mathrm{mds} .10248$.

Rascol, Olivier (2009). "\&quot;Disease-modification\&quot; trials in Parkinson disease: target populations, endpoints and study design." In: Neurology 72.7 Suppl, pp. 51-8. ISSN: 1526-632X. DOI: 10 . 1212 /WNL . Ob013e318199049e. URL: http : //www.ncbi.nlm.nih.gov/pubmed/19221315.

Reeve, Amy, Eve Simcox, and Doug Turnbull (2014). "Ageing and Parkinson's disease: Why is advancing age the biggest risk factor?" In: Ageing Research Reviews 14, pp. 19-30. ISSN: 15681637. DOI: 10 . 1016/ j . arr . 2014 . 01 .004. URL: http: //www .ncbi.nlm.nih.gov/pubmed/24503004http: //www . pubmedcentral. nih . gov / articlerender . fcgi ? artid=PMC3989046http : / / linkinghub . elsevier . com/retrieve/pii/S1568163714000051.

Rempel, D et al. (1994). "A method of measuring fingertip loading during keyboard use." In: Journal of biomechanics 27.8, pp. 1101-4. ISSN: 0021-9290. URL: http: // www.ncbi.nlm.nih.gov/pubmed/8089165.

Revicki, Dennis et al. (2008). "Recommended methods for determining responsiveness and minimally important differences for patient-reported outcomes". In: Journal of Clinical Epidemiology 61.2, pp. 102-109. ISSN: 08954356. DOI: 10.1016/ j . jclinepi . 2007 .03 .012. URL: http : / / www . ncbi .nlm.nih.gov/pubmed / 18177782https://linkinghub.elsevier.com/retrieve/pii/S0895435607001199.

Ricciardi, Lucia et al. (2016). "Rehabilitation of hypomimia in Parkinson's disease: a feasibility study of two different approaches". In: Neurological Sciences 37.3, pp. 431-436. ISSN: 1590-1874. DOI: 10 . 1007 / s10072-015-2421-9. URL: http : //www .ncbi.nlm.nih. gov/pubmed/26590993http: //link. springer . com/10 . 1007/s10072-015-2421-9.

Robinson, J.A. et al. (1998). “Computer user verification using login string keystroke dynamics". In: IEEE Transactions on Systems, Man, and Cybernetics - Part A: Systems and Humans 28.2, pp. 236-241. ISSN: 10834427. DOI: 10.1109/3468.661150. URL: http://ieeexplore.ieee.org/document/661150/.

Rodriguez-Oroz, Maria C et al. (2009). "Initial clinical manifestations of Parkinson's disease: features and pathophysiological mechanisms". In: The Lancet Neurology 
8.12, pp. 1128-1139. ISSN: 1474-4422. DOI: 10 . 1016/S1474-4422(09) 70293 - 5. URL: https://www. sciencedirect.com/science/article/pii/S1474442209702935.

Rooden, Stephanie M. van et al. (2010). “The identification of Parkinson's disease subtypes using cluster analysis: A systematic review". In: Movement Disorders 25.8, pp. 969-978. ISSN: 08853185. DOI: 10.1002/mds . 23116. URL: http: //www . ncbi.nlm.nih. gov/pubmed/20535823http://doi . wiley.com/10 . 1002/mds . 23116.

Ross, G. Webster et al. (2008). "Association of olfactory dysfunction with risk for future Parkinson's disease". In: Annals of Neurology 63.2, pp. 167-173. ISSN: 03645134. DOI: 10 . 1002 / ana . 21291. URL: http : / / www . ncbi . nlm . nih . gov / pubmed / 18067173http://doi.wiley.com/10.1002/ana.21291.

Ross, G. Webster et al. (2012). "Pre-motor features of Parkinson's disease: the HonoluluAsia Aging Study experience". In: Parkinsonism \& Related Disorders 18, S199-S202. ISSN: 13538020. DOI: 10.1016/S1353-8020(11)70062-1. URL: http://www.ncbi. nlm.nih.gov/pubmed/22166434http://linkinghub.elsevier.com/retrieve/ $\mathrm{pii} / \mathrm{S} 1353802011700621$.

Rovini, Erika, Carlo Maremmani, and Filippo Cavallo (2017). "How Wearable Sensors Can Support Parkinson's Disease Diagnosis and Treatment: A Systematic Review." In: Frontiers in neuroscience 11, p. 555. ISSN: 1662-4548. DOI: 10 . 3389/ fnins. 2017.00555. URL: http://www.ncbi.nlm.nih.gov/pubmed/29056899http: //www . pubmedcentral.nih.gov/articlerender.fcgi?artid=PMC5635326.

Rovini, Erika et al. (2018). "Comparative Motor Pre-clinical Assessment in Parkinson's Disease Using Supervised Machine Learning Approaches". In: Annals of Biomedical Engineering 46.12, pp. 2057-2068. ISSN: 0090-6964. DOI: 10.1007/s10439018-2104-9. URL: http://link. springer.com/10.1007/s10439-018-2104-9.

Rovini, Erika et al. (2019). "DAPHNE: A Novel e-Health System for the Diagnosis and the Treatment of Parkinson's Disease". In: pp. 271-288. DOI: 10 . 1007/9783-030-04672-9\{\_\}19. URL: http://link. springer.com/10.1007/978-3-03004672-9_19.

Roy, Serge H. et al. (2013). "High-resolution tracking of motor disorders in Parkinson's disease during unconstrained activity". In: Movement Disorders 28.8, pp. 10801087. ISSN: 08853185. DOI: 10.1002/mds . 25391. URL: http://www.ncbi .nlm.nih. gov/pubmed/23520058http: / / www . pubmedcentral .nih.gov/articlerender . fcgi?artid=PMC6267776http://doi.wiley.com/10.1002/mds . 25391.

Ruder, Sebastian (2017). "An Overview of Multi-Task Learning in Deep Neural Networks". In: URL: http://arxiv.org/abs/1706. 05098.

Ruonala, V. et al. (2013). "EMG signal morphology in essential tremor and Parkinson's disease". In: 2013 35th Annual International Conference of the IEEE Engineering in Medicine and Biology Society (EMBC). Vol. 2013. IEEE, pp. 5765-5768. ISBN: 9781-4577-0216-7. DOI: 10 .1109/EMBC . 2013 .6610861. URL: http://www . ncbi .nlm . nih.gov/pubmed/24111048http://ieeexplore. ieee.org/document/6610861/.

Ruonala, Verneri et al. (2015). "Autonomic nervous system response to L-dopa in patients with advanced Parkinson's disease". In: 2015 37th Annual International Conference of the IEEE Engineering in Medicine and Biology Society (EMBC). Vol. 2015. IEEE, pp. 6162-6165. ISBN: 978-1-4244-9271-8. DOI: 10.1109/EMBC . 2015.7319799. URL: http: //www .ncbi.nlm.nih.gov/pubmed/26737699http: //ieexplore . ieee.org/document/7319799/.

Sama, A. et al. (2012). “Dyskinesia and motor state detection in Parkinson's Disease patients with a single movement sensor". In: 2012 Annual International Conference of the IEEE Engineering in Medicine and Biology Society. Vol. 2012. IEEE, pp. 11941197. ISBN: 978-1-4577-1787-1. DOI: 10 . 1109/EMBC . 2012 . 6346150. URL: http : 
//www .ncbi.nlm.nih.gov/pubmed/23366111http://ieeexplore. ieee.org/ document $/ 6346150 /$.

Samà, Albert et al. (2014). "A double closed loop to enhance the quality of life of Parkinson's Disease patients: REMPARK system." In: Studies in health technology and informatics 207, pp. 115-24. ISSN: 0926-9630. URL: http: // www . ncbi . nlm . nih.gov/pubmed/25488217.

Sánchez-Ferro, Álvaro et al. (2016a). "New methods for the assessment of Parkinson's disease (2005 to 2015): A systematic review". In: Movement Disorders 31.9, pp. 1283-1292. ISSN: 08853185. DOI: 10 . 1002 /mds . 26723. URL: http : / / doi . wiley.com/10.1002/mds. 26723.

- (2016b). "New methods for the assessment of Parkinson's disease (2005 to 2015): A systematic review". In: Movement Disorders 31.9, pp. 1283-1292. ISSN: 08853185. DOI: 10.1002/mds .26723. URL: http://doi . wiley. com/10.1002/mds . 26723.

Sánchez-Ferro, Álvaro et al. (2018). "Minimal Clinically Important Difference for UPDRS-III in Daily Practice". In: Movement Disorders Clinical Practice. ISSN: 23301619. DOI: 10.1002/mdc3.12632. URL: http://doi .wiley.com/10.1002/mdc3. 12632.

Sant'Anna, Anita, Arash Salarian, and Nicholas Wickstrom (2011). "A new measure of movement symmetry in early Parkinson's disease patients using symbolic processing of inertial sensor data". In: IEEE Transactions on Biomedical Engineering 58.7, pp. 2127-2135. ISSN: 0018-9294. DOI: 10 . 1109/ TBME . 2011 . 2149521. URL: http://www.ncbi.nlm.nih.gov/pubmed/21536527http://ieeexplore. ieee. org/document/5762333/.

Schapira, A. H. V. (2008). "Progress in neuroprotection in Parkinson's disease". In: European Journal of Neurology 15.s1, pp. 5-13. ISSN: 1351-5101. DOI: $10.1111 / \mathrm{j}$. 1468 - 1331 . 2008 . 02055 .x. URL: http: / / www . ncbi .nlm . nih.gov/pubmed / 18353131http://doi.wiley.com/10.1111/j.1468-1331.2008.02055.x.

Schapira, Anthony H. and Peter Jenner (2011). "Etiology and pathogenesis of Parkinson's disease". In: Movement Disorders 26.6, pp. 1049-1055. ISSN: 08853185. DOI: 10.1002/mds . 23732. URL: http://www.ncbi.nlm.nih.gov/pubmed/21626550http: //doi.wiley.com/10.1002/mds. 23732.

Schapira, Anthony H V and Eduardo Tolosa (2010). "Molecular and clinical prodrome of Parkinson disease: implications for treatment". In: Nature Reviews Neurology 6.6, pp. 309-317. ISSN: 1759-4758. DOI: 10 . 1038/nrneurol . 2010 . 52. URL: http://www. nature.com/articles/nrneurol.2010.52.

Schapira, Anthony H. V. (Anthony Henry Vernon), Andreas Hartmann, and Yves. Agid (2009). Parkinsonian disorders in clinical practice. Wiley-Blackwell, p. 196. ISBN: 1444306391.

Scheer, Frank A. J. L. et al. (2008). “An Endogenous Circadian Rhythm in Sleep Inertia Results in Greatest Cognitive Impairment upon Awakening during the Biological Night". In: Journal of Biological Rhythms 23.4, pp. 353-361. ISSN: 0748-7304. DOI: 10.1177/0748730408318081. URL: http://www.ncbi.nlm.nih.gov/pubmed/ 18663242http: //www . pubmedcentral . nih . gov/articlerender.fcgi?artid= PMC3130065http://journals. sagepub.com/doi/10.1177/0748730408318081.

Schenck, C H, S R Bundlie, and M W Mahowald (1996). "Delayed emergence of a parkinsonian disorder in $38 \%$ of 29 older men initially diagnosed with idiopathic rapid eye movement sleep behaviour disorder." In: Neurology 46.2, pp. 388-93. ISSN: 0028-3878. URL: http://www.ncbi.nlm.nih.gov/pubmed/8614500.

Schieber, Marc H. and Marco Santello (2004). "Hand function: peripheral and central constraints on performance". In: Journal of Applied Physiology 96.6, pp. 2293-2300. ISSN: 8750-7587. DOI: 10 .1152/ japplphysiol . 01063 . 2003. URL: http : / /www . 
ncbi.nlm.nih . gov/pubmed/15133016http: // www · physiology . org/doi/10 . 1152/japplphysiol.01063.2003.

Schneider, Susanne A. et al. (2012). "A study of subtle motor signs in early Parkinson's disease". In: Movement Disorders 27.12, pp. 1563-1566. ISSN: 08853185. DOI: 10.1002/mds.25161. URL: http://www.ncbi.nlm.nih.gov/pubmed/23032728http: //doi.wiley.com/10.1002/mds.25161.

Schrag, A, Y Ben-Shlomo, and N Quinn (2002). "How valid is the clinical diagnosis of Parkinson's disease in the community?" In: Journal of neurology, neurosurgery, and psychiatry 73.5, pp. 529-534. ISSN: 0022-3050. DOI: 10.1136/ JNNP. 73.5.529. URL: http://www.ncbi.nlm.nih.gov/pubmed/12397145http://www.pubmedcentral . nih.gov/articlerender. fcgi?artid=PMC1738115.

Schrag, Anette et al. (2006). "Minimal clinically important change on the unified Parkinson's disease rating scale". In: Movement Disorders 21.8, pp. 1200-1207. ISSN: 0885-3185. DOI: 10 . 1002/mds . 20914. URL: http : / www . ncbi . nlm . nih . gov/pubmed/16673410http://doi.wiley.com/10.1002/mds. 20914.

Schuepbach, W.M.M. et al. (2013). “Neurostimulation for Parkinson's Disease with Early Motor Complications". In: New England Journal of Medicine 368.7, pp. 610622. ISSN: 0028-4793. DOI: 10 .1056/NEJMoa1205158. URL: http: //www . nejm. org/ doi/10.1056/NEJMoa1205158.

Shaw, Leslie M et al. (2007). "Biomarkers of neurodegeneration for diagnosis and monitoring therapeutics". In: Nature Reviews Drug Discovery 6.4, pp. 295-303. ISSN: 1474-1776. DOI: 10.1038/nrd2176. URL: http: //www . nature . com/articles/ nrd2176.

Sheather, S. J. and M. Chris Jones (1991). "A Reliable Data-Based Bandwidth Selection Method for Kernel Density Estimation”. In: 53, pp. 683-690. ISSN: 0035-9246. DOI: $10.2307 / 2345597$.

Sherer, Todd B. et al. (2012). "Overcoming obstacles in Parkinson's disease". In: Movement Disorders 27.13, pp. 1606-1611. ISSN: 08853185. DOI: 10 . 1002 / mds . 25260. URL: http://doi.wiley.com/10.1002/mds. 25260.

Sherman, Rachel E. et al. (2016). "Real-World Evidence - What Is It and What Can It Tell Us?" In: New England Journal of Medicine 375.23, pp. 2293-2297. ISSN: 00284793. DOI: 10.1056/NEJMsb1609216. URL: http://www. nejm.org/doi/10.1056/ NEJMsb1609216.

Shimoyama, Ichiro, Toshiaki Ninchoji, and Kenichi Uemura (1990). “The FingerTapping Test". In: Archives of Neurology 47.6, p. 681. ISSN: 0003-9942. DOI: 10 . 1001 /archneur . 1990.00530060095025 . URL: http : / / archneur . jamanetwork . com/article. aspx?doi=10.1001/archneur . 1990.00530060095025.

Shprecher, David et al. (2012). "Willingness of Parkinson's disease patients to participate in research using internet-based technology." In: Telemedicine journal and ehealth : the official journal of the American Telemedicine Association 18.9, pp. 684-687. ISSN: 1556-3669. DOI: 10.1089/tmj . 2011.0276. URL: http://www .ncbi.nlm.nih. gov/pubmed/22954069http : / / www . pubmedcentral . nih.gov/articlerender . fcgi?artid=PMC3491628.

Shulman, Lisa M. et al. (2010). "The Clinically Important Difference on the Unified Parkinson's Disease Rating Scale". In: Archives of Neurology 67.1, pp. 64-70. ISSN: 0003-9942. DOI: 10 . 1001/archneurol . 2009 . 295. URL: http: / /ww . ncbi . nlm . nih.gov/pubmed/20065131http://archneur.jamanetwork.com/article.aspx? doi=10.1001/archneurol.2009.295.

Simieli, Lucas et al. (2018). "Variability of crossing phase in older people with Parkinson's disease is dependent of obstacle height". In: Scientific Reports 8.1, p. 14852. 
ISSN: 2045-2322. DOI: 10 . 1038/s41598-018-33312-2. URL: http: //www . nature . com/articles/s41598-018-33312-2.

Spyers-Ashby, Julia M and Maria J Stokes (2000). "Reliability of tremor measurements using a multidimensional electromagnetic sensor system". In: Clinical Rehabilitation 14.4, pp. 425-432. ISSN: 0269-2155. DOI: 10.1191/0269215500cr328oa. URL: http://journals . sagepub. com/doi/10.1191/0269215500cr328oa.

Stanzione, Paolo and Domenicantonio Tropepi (2011). "Drugs and clinical trials in neurodegenerative diseases". In: Annali dell'Istituto Superiore di Sanità 47, pp. 4954. ISSN: 0021-2571. DOI: $10.4415 / \operatorname{ANN}\left\{\backslash_{-}\right\} 11\left\{\backslash_{-}\right\} 01\left\{\backslash_{-}\right\} 11$. URL: https : / / www. scielosp.org/scielo . php?pid=S0021-25712011000100011\&script=sci arttext\&tlng=es.

Stocchi, Fabrizio and C. Warren Olanow (2013). "Obstacles to the Development of a Neuroprotective Therapy for Parkinson's Disease". In: Movement Disorders 28.1, pp. 3-7. ISSN: 08853185. DOI: 10.1002/mds . 25337. URL: http://www . ncbi .nlm. nih.gov/pubmed/23390094http://doi.wiley.com/10.1002/mds. 25337.

Stolerman, Ian P. and Lawrence H. Price (2011). Encyclopedia of psychopharmacology. ISBN: 9783642361715.

Streffer, Johannes R. et al. (2012). "Prerequisites to launch neuroprotective trials in Parkinson's disease: An industry perspective". In: Movement Disorders 27.5, pp. 651655. ISSN: 08853185. DOI: 10.1002/mds . 25017. URL: http://www.ncbi .nlm.nih . gov/pubmed/22508283http://doi.wiley.com/10.1002/mds . 25017.

Sun, Jimeng and Chandan K. Reddy (2013). "Big data analytics for healthcare". In: Proceedings of the 19th ACM SIGKDD international conference on Knowledge discovery and data mining - KDD '13. New York, New York, USA: ACM Press, p. 1525. ISBN: 9781450321747. DOI: 10 . 1145/2487575 . 2506178. URL: http : / / dl . acm . org/citation. cfm?doid=2487575.2506178.

Suzuki, Masahiko, Hiroshi Mitoma, and Mitsuru Yoneyama (2017). "Quantitative Analysis of Motor Status in Parkinson's Disease Using Wearable Devices: From Methodological Considerations to Problems in Clinical Applications." In: Parkinson's disease 2017, p. 6139716. ISSN: 2090-8083. DOI: 10 .1155/2017/6139716. URL: http://www.ncbi.nlm.nih.gov/pubmed/28607801http://www . pubmedcentral. nih.gov/articlerender.fcgi?artid=PMC5451764.

Takahashi, Kayoko et al. (2016). "Quality of life in people with Parkinson's disease: the relevance of social relationships and communication." In: Journal of physical therapy science 28.2, pp. 541-546. ISSN: 0915-5287. DOI: 10 . 1589 / jpts . 28 . 541. URL: http : / / www . ncbi . nlm . nih . gov / pubmed/27065542http : / / www . pubmedcentral .nih.gov/articlerender.fcgi?artid=PMC4793007.

Tanner, C M and S M Goldman (1996). “Epidemiology of Parkinson's disease." In: Neurologic clinics 14.2, pp. 317-35. ISSN: 0733-8619. DOI: 10. 1016/S0733-8619(05) 70259-0. URL: http://www.ncbi.nlm.nih.gov/pubmed/8827174.

Tarakad, Arjun and Joseph Jankovic (2017). “Diagnosis and Management of Parkinson's Disease". In: Seminars in Neurology 37.02, pp. 118-126. DOI: 10.1055 / s 0037-1601888. URL: http : / www . ncbi . nlm . nih . gov/pubmed/28511252http : //www.ncbi.nlm.nih.gov/pubmed/28511252.

Taylor Tavares, Ana Lisa et al. (2005a). "Quantitative measurements of alternating finger tapping in Parkinson's disease correlate with UPDRS motor disability and reveal the improvement in fine motor control from medication and deep brain stimulation". In: Movement Disorders 20.10, pp. 1286-1298. ISSN: 0885-3185. DOI: 10.1002/mds . 20556. URL: http://doi.wiley.com/10.1002/mds. 20556.

Taylor Tavares, Ana Lisa et al. (2005b). “Quantitative measurements of alternating finger tapping in Parkinson's disease correlate with UPDRS motor disability and 
reveal the improvement in fine motor control from medication and deep brain stimulation." In: Movement disorders : official journal of the Movement Disorder Society 20.10, pp. 1286-98. DOI: $10.1002 / \mathrm{mds} .20556$.

Thanvi, B R and T C N Lo (2004). "Long term motor complications of levodopa: clinical features, mechanisms, and management strategies." In: Postgraduate medical journal 80.946, pp. 452-458. ISSN: 0032-5473. DOI: 10 . $1136 / \mathrm{pgmj}$. 2003. 013912. URL: http://www .ncbi.nlm.nih . gov/pubmed/15299154http: //www . pubmedcentral . nih.gov/articlerender . fcgi?artid=PMC1743071.

“The Unified Parkinson's Disease Rating Scale (UPDRS): Status and recommendations" (2003). In: Movement Disorders 18.7, pp. 738-750. ISSN: 0885-3185. DOI: 10. 1002/mds.10473. URL: http://doi.wiley.com/10.1002/mds. 10473.

Tibar, Houyam et al. (2018). “Non-Motor Symptoms of Parkinson's Disease and Their Impact on Quality of Life in a Cohort of Moroccan Patients". In: Frontiers in Neurology 9. ISSN: 1664-2295. DOI: 10 . 3389/fneur . 2018 .00170. URL: http: //journal.frontiersin.org/article/10.3389/fneur.2018.00170/full.

Tiffin, Joseph and E. J. Asher (1948). "The Purdue Pegboard: norms and studies of reliability and validity." In: Journal of Applied Psychology 32.3, pp. 234-247. ISSN: 1939-1854. DOI: 10 . 1037/h0061266. URL: http: / / doi . apa . org/getdoi . cfm? doi $=10.1037 / \mathrm{h} 0061266$.

Titova, Nataliya, Peter Jenner, and K Ray Chaudhuri (2017). “The Future of Parkinson's Treatment - Personalised and Precision Medicine". In: European Neurological Review 12.01, p. 15. ISSN: 1758-3837. DOI: 10.17925/ENR. 2017.12.01.15. URL: http://www . touchneurology.com/articles/future-parkinson-s-treatmentpersonalised-and-precision-medicine.

Topol, Eric (2016). "Digital medicine: empowering both patients and clinicians". In: The Lancet 388.10046, pp. 740-741. ISSN: 0140-6736. DOI: 10 . 1016/ S0140 6736 (16)31355-1. URL: https : //www . sciencedirect . com/science/article/ pii/S0140673616313551.

Torous, John et al. (2016). "New Tools for New Research in Psychiatry: A Scalable and Customizable Platform to Empower Data Driven Smartphone Research." In: JMIR mental health 3.2, e16. ISSN: 2368-7959. DOI: 10 .2196/mental . 5165. URL: http : //mental . jmir . org/2016/2/e16/http : //www . ncbi .nlm.nih . gov/ pubmed/27150677http: //www . pubmedcentral . nih.gov/articlerender.fcgi? artid=PMC4873624.

Tsanas, Athanasios et al. (2012). "Novel speech signal processing algorithms for high-accuracy classification of Parkinson's disease." In: IEEE transactions on biomedical engineering 59.5, pp. 1264-71. DOI: 10.1109/TBME. 2012.2183367.

Tsipouras, Markos G. et al. (2012). “An automated methodology for levodopa-induced dyskinesia: Assessment based on gyroscope and accelerometer signals". In: Artificial Intelligence in Medicine 55.2, pp. 127-135. ISSN: 09333657. DOI: 10.1016 / j . artmed . 2012 .03 . 003. URL: http : / / www . ncbi . nlm . nih . gov/pubmed / 22484102http://linkinghub.elsevier.com/retrieve/pii/S0933365712000322.

Tsipouras, Markos G et al. (2014). "A wearable system for long-term ubiquitous monitoring of common motor symptoms in patients with Parkinson's disease". In: IEEE-EMBS International Conference on Biomedical and Health Informatics (BHI). IEEE, pp. 173-176. ISBN: 978-1-4799-2131-7. DOI: 10 . 1109/BHI . 2014 . 6864332. URL: http://ieeexplore. ieee.org/document/6864332/.

TypingDNA - Typing Biometrics, Keystroke Dynamics. URL: https : //www . typingdna . $\mathrm{com} /$.

Tzallas, Alexandros T et al. (2014). "PERFORM: a system for monitoring, assessment and management of patients with Parkinson's disease." In: Sensors (Basel, 
Switzerland) 14.11, pp. 21329-21357. ISSN: 1424-8220. DOI: $10.3390 /$ s141121329. URL: http://www.ncbi.nlm.nih.gov/pubmed/25393786http://www . pubmedcentral . nih.gov/articlerender.fcgi?artid=PMC4279536.

Vaiciukynas, Evaldas et al. (2017). “Detecting Parkinson's disease from sustained phonation and speech signals". In: PLOS ONE 12.10. Ed. by Enzo Pasquale Scilingo, e0185613. ISSN: 1932-6203. DOI: 10 .1371/journal . pone .0185613. URL: https : //dx.plos.org/10.1371/journal.pone.0185613.

Vir V. Phoha et al. (2008). "Hidden Markov Model HMM-Based User Authentication Using Keystroke Dynamics". In: URL: https : / patents . google.com/patent/ US20090328200.

Witt, Suzanne T., Angela R. Laird, and M. Elizabeth Meyerand (2008). "Functional neuroimaging correlates of finger-tapping task variations: An ALE meta-analysis". In: NeuroImage 42.1, pp. 343-356. ISSN: 10538119. DOI: $10.1016 / \mathrm{j}$. neuroimage . 2008.04.025. URL: http://www.ncbi.nlm.nih.gov/pubmed/18511305http:// www . pubmedcentral . nih . gov/articlerender . fcgi ?artid=PMC2592684https : //linkinghub.elsevier.com/retrieve/pii/S1053811908003236.

Worringham, Charles J et al. (2006). "Predictors of driving assessment outcome in Parkinson's disease". In: Movement Disorders 21.2, pp. 230-235. ISSN: 0885-3185. DOI: 10.1002/mds .20709. URL: http://doi . wiley.com/10.1002/mds . 20709.

Wright, Alexis et al. (2012). "Clinimetrics corner: a closer look at the minimal clinically important difference (MCID)". In: Journal of Manual \& Manipulative Therapy 20.3, pp. 160-166. ISSN: 1066-9817. DOI: 10.1179/2042618612Y.0000000001. URL: http://www.ncbi.nlm.nih.gov/pubmed/23904756http://www. pubmedcentral. nih. gov/articlerender . fcgi ? artid=PMC3419574http: / / ww . tandfonline . $\mathrm{com} / \mathrm{doi} / \mathrm{full} / 10.1179 / 2042618612$ Y.0000000001.

Wright, JustinM et al. (2017). "Evolution of the digital biomarker ecosystem". In: Digital Medicine 3.4, p. 154. ISSN: 2226-8561. DOI: $10.4103 /$ digm.digm\{\_\}35\{\_ \}17. URL: http://www .digitmedicine.com/text.asp?2017/3/4/154/228663.

Yang, Ke et al. (2016). "Objective and quantitative assessment of motor function in Parkinson's disease-from the perspective of practical applications." In: Annals of translational medicine 4.5, p. 90. ISSN: 2305-5839. DOI: 10 . 21037 / atm . 2016 . 03. 09. URL: http : / / www . ncbi . nlm . nih . gov/ pubmed / 27047949http : / / www . pubmedcentral . nih.gov/articlerender. fcgi?artid=PMC4791329.

Yen, Chang-Yi et al. (2011). "Effects of Virtual Reality-Augmented Balance Training on Sensory Organization and Attentional Demand for Postural Control in People With Parkinson Disease: A Randomized Controlled Trial". In: Physical Therapy 91.6, pp. 862-874. ISSN: 0031-9023. DOI: 10 . 2522 / ptj . 20100050. URL: https : //academic.oup.com/ptj/article-lookup/doi/10.2522/ptj. 20100050.

Yong, Shereen, Weng Kin Lai, and George Goghill (2004). "Weightless Neural Networks for Typing Biometrics Authentication". In: Springer, Berlin, Heidelberg, pp. 284-293. DOI: 10 . 1007 / $978-3-540-30133-2\left\{\backslash \_\right\} 37$. URL: http : / / link . springer.com/10.1007/978-3-540-30133-2_37.

$\mathrm{Yu}$, Enzhe and Sungzoon Cho (2004). "Keystroke dynamics identity verification-its problems and practical solutions". In: Computers $\mathcal{E}$ Security 23.5, pp. 428-440. ISSN: 01674048. DOI: $10.1016 / j$. cose . 2004.02 .004. URL: https : //linkinghub. elsevier.com/retrieve/pii/S0167404804000963.

Zackrisson, Theresa et al. (2013). "The Discriminating Properties of an Optoelectronic Movement Analysis Method in Patients With Parkinsonism". In: Journal of Motor Behavior 45.5, pp. 415-422. ISSN: 0022-2895. DOI: 10.1080/00222895 . 2013. 815152. URL: https : / / www . tandfonline . com/doi / full/10 . 1080/00222895. 2013.815152. 
Zhan, Andong et al. (2018). "Using Smartphones and Machine Learning to Quantify Parkinson Disease Severity". In: JAMA Neurology 75.7, p. 876. ISSN: 2168-6149. DOI: 10 . 1001/ jamaneurol . 2018 . 0809. URL: http : / / archneur . jamanetwork . com/article.aspx?doi=10.1001/jamaneurol . 2018.0809.

Zhang, Jiuquan et al. (2015). "Akinetic-rigid and tremor-dominant Parkinson's disease patients show different patterns of intrinsic brain activity". In: Parkinsonism $\mathcal{E}$ Related Disorders 21.1, pp. 23-30. ISSN: 1353-8020. DOI: 10 . 1016/J . PARKRELDIS . 2014.10.017. URL: https://www . sciencedirect.com/science/article/pii/ S1353802014004027. 